Supporting Information

\title{
Diversity-Oriented Synthesis of 3-lodochromones and Heteroatom Analogues via ICl-Induced Cyclization
}

Chengxiang Zhou, Anton V. Dubrovsky, and Richard C. Larock*

Department of Chemistry, Iowa State University, Ames, Iowa 50011

larock@iastate.edu

Table of Contents $\quad$ S1

General Procedures $\quad$ S2-S4

$\begin{array}{ll}\text { Characterization data } & \text { S5-S22 }\end{array}$

$\begin{array}{ll}\text { References } & \text { S22 }\end{array}$

${ }^{1} \mathrm{H}$ and ${ }^{13} \mathrm{C}$ NMR spectra $\quad$ S23-S122 


\section{General}

The ${ }^{1} \mathrm{H}$ and ${ }^{13} \mathrm{C}$ NMR spectra were recorded at $300 \mathrm{MHz}$ or $400 \mathrm{MHz}$ and $75 \mathrm{MHz}$ or $100 \mathrm{MHz}$, respectively. Thin layer chromatography was performed using commercially prepared 60-mesh silica gel plates, and visualization was effected with short wavelength UV light $(254 \mathrm{~nm})$. High resolution mass spectra were recorded on a double focusing magnetic sector mass spectrometer using EI at $70 \mathrm{eV}$. All melting points are uncorrected. All reagents were used as obtained commercially. The palladium salts were donated by Johnson Matthey Inc. and Kawaken Fine Chemicals Co., Ltd. The arylboronic acids were donated by Frontier Scientific Co., Ltd.

\section{General procedure for the preparation of ynones}<smiles>[Y]c1ccc(OC)c(C(=O)Cl)c1</smiles><smiles>[R]C#CC[13CH]C</smiles><smiles>[R]C#CC(=O)c1ccccc1OC</smiles>

Method A (Sonogashira route): To a flask were added sequentially $\mathrm{CuI}(0.02$ $\mathrm{mmol}), \mathrm{PdCl}_{2}(\mathrm{PPh} 3)_{2}(0.04 \mathrm{mmol})$, terminal acetylene $(2.6 \mathrm{mmol})$, the benzoyl chloride $(2 \mathrm{mmol})$ and triethylamine $(20 \mathrm{~mL})$. The resulting mixture was flushed with $\mathrm{N}_{2}$ and allowed to stir at room temperature or at $50{ }^{\circ} \mathrm{C}$ overnight. Water $(15$ $\mathrm{mL}$ ) was added, and the aqueous layer was extracted with ethyl ether 3 times (25 $\mathrm{mL}$ each time). The organic layers were combined, dried and concentrated under reduced pressure. The residue was then purified by column chromatography on silica gel to afford the desired alkynone.<smiles>Cc1ccccc1C=O</smiles>

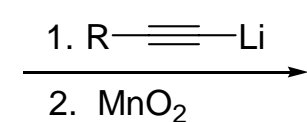<smiles>[R]C#CC(=O)c1ccccc1C</smiles>

Method B (oxidation route): The benzaldehyde $(2.0 \mathrm{mmol})$ was placed in a flask and dissolved in $15 \mathrm{~mL}$ of dry, oxygen-free THF. The flask was flushed with $\mathrm{N}_{2}$ and cooled to $-78{ }^{\circ} \mathrm{C}$. The lithium acetylide $(2.2 \mathrm{mmol}$ in $20 \mathrm{~mL}$ of THF solution) was then added dropwise. The resulting mixture was stirred at $-78{ }^{\circ} \mathrm{C}$ for $30 \mathrm{~min}$ 
and allowed to gradually warm to room temperature. The reaction mixture was quenched by satd aq $\mathrm{NH}_{4} \mathrm{Cl}(20 \mathrm{~mL})$ and extracted with ethyl ether 3 times $(25 \mathrm{~mL}$ each time). The organic layers were combined, dried and concentrated under reduced pressure. The residue was purified by column chromatography on silica gel to afford the desired secondary alcohol. The alcohol was then dissolved in $\mathrm{CHCl}_{3}$ $(25 \mathrm{~mL})$ and activated $\mathrm{MnO}_{2}(6 \mathrm{mmol})$ was added to the solution. The suspension was refluxed for $2 \mathrm{~h}$, the solution was cooled and filtered through a pad of celite, and the filtrate was concentrated to afford the desired alkynone.

\section{General procedure for the synthesis of 3-iodochromones and heteroatom analogues (Table 1)}
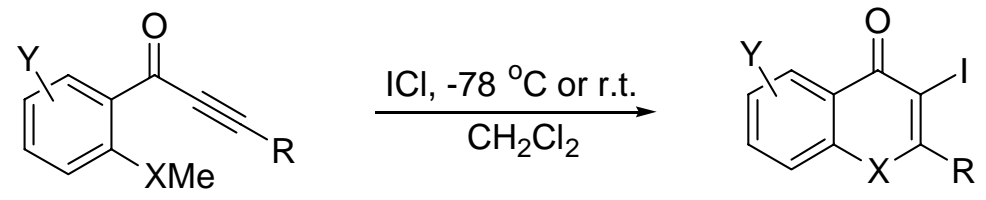

$$
\mathrm{X}=\mathrm{O}, \mathrm{S}, \mathrm{NMe}
$$

The alkynone $(0.25 \mathrm{mmol})$ and $\mathrm{ICl}(0.375 \mathrm{mmol})$ are each dissolved in $1.5 \mathrm{~mL}$ of dry $\mathrm{CH}_{2} \mathrm{Cl}_{2}$ in separate vials and cooled to $-78{ }^{\circ} \mathrm{C}$. The $\mathrm{ICl}$ solution is added dropwise to the alkynone solution over approximately $1 \mathrm{~min}$ and the reaction is stirred at the indicated temperature for the indicated time. To the reaction mixture is then added 2 $\mathrm{mL}$ of satd aq $\mathrm{Na}_{2} \mathrm{~S}_{2} \mathrm{O}_{3}$ solution and then $20 \mathrm{~mL}$ of satd $\mathrm{NaCl}$ solution. The resulting mixture was extracted three times with ethyl ether ( $30 \mathrm{~mL}$ each time). The combined organic layers were dried over anhydrous $\mathrm{MgSO}_{4}$ and the solvent was evaporated under reduced pressure. The residue provided the ${ }^{1} \mathrm{H}$ NMR pure (>95\% purity) product in most cases, but sometimes the product was isolated by chromatography on a silica gel column. 


\section{Preparation of iodochromone 2 on a multi-gram scale without the use of column chromatography}<smiles>COc1ccccc1C=O</smiles>

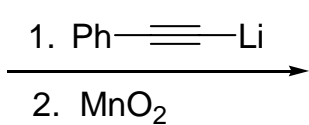

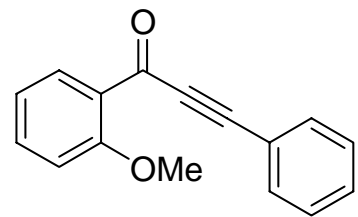

1

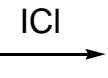<smiles>O=c1c(I)c(-c2ccccc2)oc2ccccc12</smiles>

98\% overall yield

2-Methoxybenzaldehyde $(2.72 \mathrm{~g}, 20 \mathrm{mmol})$ was added to a $500 \mathrm{~mL}$ round bottom flask and dissolved in $250 \mathrm{~mL}$ of dry, oxygen-free THF. The flask was flushed with $\mathrm{N}_{2}$ and allowed to cool to $-78{ }^{\circ} \mathrm{C}$. Lithium phenylacetylide $(20 \mathrm{~mL}$ of a $1.0 \mathrm{M}$ solution in THF) was then added dropwise under $\mathrm{N}_{2}$. The resulting mixture was stirred at $-78{ }^{\circ} \mathrm{C}$ for $30 \mathrm{~min}$ and allowed to gradually warm to room temperature. The reaction mixture was quenched by satd aq $\mathrm{NH}_{4} \mathrm{Cl}(200 \mathrm{~mL})$ and extracted with ethyl ether 3 times (100 $\mathrm{mL}$ each time). The organic layers were combined, dried and concentrated under reduced pressure. The residue was then dissolved in $\mathrm{CHCl}_{3}(250 \mathrm{~mL})$ and activated $\mathrm{MnO}_{2}$ (3.6 g, $40 \mathrm{mmol}$ ) was added. The suspension was refluxed for $2 \mathrm{~h}$, the solution was cooled and filtered through a pad of celite, and the filtrate was concentrated to afford the desired alkynone $\mathbf{1}$. The alkynone 1 was then dissolved in $50 \mathrm{~mL}$ of dry $\mathrm{CH}_{2} \mathrm{Cl}_{2}$ and cooled to $-78{ }^{\circ} \mathrm{C}$. $\mathrm{ICl}(4.06 \mathrm{~g}, 25 \mathrm{mmol})$ was dissolved in $200 \mathrm{~mL}$ of dry $\mathrm{CH}_{2} \mathrm{Cl}_{2}$ and cooled to $-78{ }^{\circ} \mathrm{C}$. The $\mathrm{ICl}$ solution was slowly added to the alkyne dropwise over a period of $15 \mathrm{~min}$. The reaction was then allowed to slowly warm up to room temperature. To the reaction mixture was added $50 \mathrm{~mL}$ of satd aq $\mathrm{Na}_{2} \mathrm{~S}_{2} \mathrm{O}_{3}$ solution and then $100 \mathrm{~mL}$ of satd aq $\mathrm{NaCl}$ solution. The resulting mixture was extracted 3 times with ethyl ether (100 $\mathrm{mL}$ each time). The combined organic layers were dried over anhydrous $\mathrm{MgSO}_{4}$ and the solvent was evaporated under reduced pressure. The residue (6.8 g, 98\% yield) proved to be chromone 2 (>95\% purity by ${ }^{1} \mathrm{H}$ and ${ }^{13} \mathrm{C}$ NMR analysis). 


\section{Characterization data:}

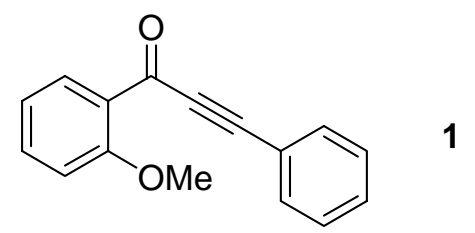

1-(2-Methoxyphenyl)-3-phenylpropynone (1). ${ }^{1} \mathrm{H} \mathrm{NMR}\left(300 \mathrm{MHz}, \mathrm{CDCl}_{3}\right) \delta 3.92$ (s, $3 \mathrm{H})$, 6.96-7.02 (m, 2H), 7.30-7.62 (m, 6H), 8.04-8.08 (m, 1H); ${ }^{13} \mathrm{C}$ NMR $(75 \mathrm{MHz}$, $\left.\mathrm{CDCl}_{3}\right) \delta 56.15,89.47,91.88,112.48,120.55,120.87,128.85,130.73,132.80,133.17$, $135.33,160.06,176.92$.

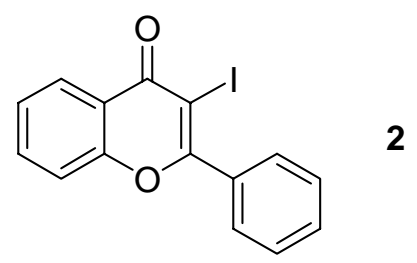

3-Iodo-2-phenylchromone (2). ${ }^{1}$ White solid: mp 127-130 ${ }^{\circ} \mathrm{C}\left(\right.$ lit. $\left.^{1} \mathrm{mp} 128{ }^{\circ} \mathrm{C}\right) ;{ }^{1} \mathrm{H}$ NMR (400 MHz, $\left.\mathrm{CDCl}_{3}\right) \delta$ 7.42-7.56 (m, 5H), 7.69-7.79 (m, 3H), 8.26-8.29 (m, 1H); ${ }^{13} \mathrm{C}$ NMR $\left(100 \mathrm{MHz}, \mathrm{CDCl}_{3}\right) \delta 88.62,115.56,117.88,120.19,126.09,126.95,128.54$, 129.68, 131.29, 134.45, 135.29, 156.08, 174.73; IR $\left(\mathrm{CDCl}_{3}, \mathrm{~cm}^{-1}\right)$ 3009, 1636; MS (EI) m/z (rel intensity) $348\left(\mathrm{M}^{+}, 48\right), 221$ (12), 135 (100); HRMS (EI) calcd for $\mathrm{C}_{15} \mathrm{H}_{9} \mathrm{IO}_{2}$ 347.9647, found: 347.9651 .

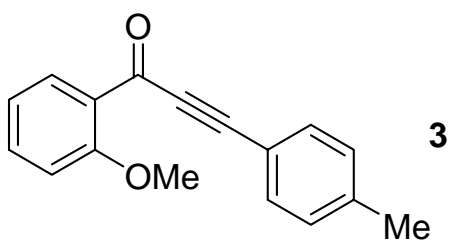

1-(2-Methoxyphenyl)-3-(4-methylphenyl)propynone (3). Light yellow solid, obtained in a $78 \%$ yield by Method A using o-anisoyl chloride and 4-ethynyltoluene: ${ }^{1} \mathrm{H}$ NMR (400 MHz, $\mathrm{CDCl}_{3}$ ) $\delta 2.29$ (s, 3H), 3.87 (s, 3H), 6.92-6.98 (m, 2H), 7.09-7.12 $(\mathrm{m}, 2 \mathrm{H}), 7.43-7.46(\mathrm{~m}, 3 \mathrm{H}), 8.00,8.04(\mathrm{~m}, 1 \mathrm{H}) ;{ }^{13} \mathrm{C} \mathrm{NMR}\left(100 \mathrm{MHz}, \mathrm{CDCl}_{3}\right) \delta 21.94$, $56.10,89.41,92.49,112.47,117.67,120.51,126.92,129.69,132.67,133.20,135.27$, $141.43,159.96,176.87$. 


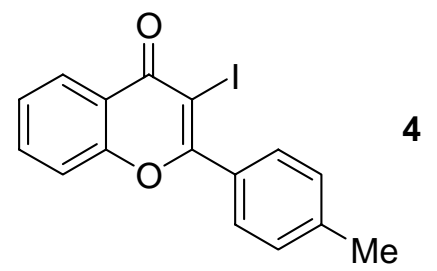

3-Iodo-2-(4-methylphenyl)chromone (4). Light yellow solid: mp $126-130{ }^{\circ} \mathrm{C} ;{ }^{1} \mathrm{H}$ NMR (300 MHz, $\left.\mathrm{CDCl}_{3}\right) \delta 2.44(\mathrm{~s}, 3 \mathrm{H}), 7.30-7.47(\mathrm{~m}, 4 \mathrm{H}), 7.65-7.70(\mathrm{~m}, 3 \mathrm{H}), 8.24(\mathrm{~d}$, $J=8.1 \mathrm{~Hz}, 1 \mathrm{H}) ;{ }^{13} \mathrm{C}$ NMR $\left(75 \mathrm{MHz}, \mathrm{CDCl}_{3}\right) \delta 21.90,88.29,117.88,120.16,126.00$, $126.88,129.19,129.69,132.38,134.38,141.80,156.05,164.88,174.73$; IR $\left(\mathrm{CDCl}_{3}\right.$, $\mathrm{cm}^{-1}$ ) 3008, 2955, 1638; MS (EI) m/z (rel intensity) $362\left(\mathrm{M}^{+}, 100\right), 235$ (21); HRMS (EI) calcd for $\mathrm{C}_{16} \mathrm{H}_{11} \mathrm{IO}_{2} 361.9804$, found: 361.9809 .

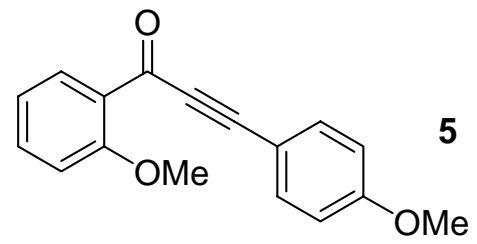

1-(2-Methoxyphenyl)-3-(4-methoxyphenyl)propynone (5). Light yellow solid, obtained in an $86 \%$ yield by Method A using $o$-anisoyl chloride and 4-ethynylanisole: ${ }^{1} \mathrm{H}$ NMR (400 MHz, $\mathrm{CDCl}_{3}$ ) $\delta 3.74$ (s, 3H), 3.88 (s, 3H), 6.81-6.84 (m, 2H), 6.92-6.99 $(\mathrm{m}, 2 \mathrm{H}), 7.43-7.52(\mathrm{~m}, 3 \mathrm{H}), 8.01(\mathrm{dd}, J=7.6,2.0 \mathrm{~Hz}, 1 \mathrm{H}) ;{ }^{13} \mathrm{C} \mathrm{NMR}\left(100 \mathrm{MHz}, \mathrm{CDCl}_{3}\right)$ $\delta 55.62,56.11,89.45,93.06,112.43,112.51,114.58,120.49,127.01,132.61,135.12$, $135.21,159.87,161.72,176.92$.

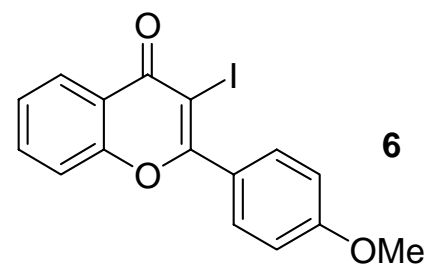

3-Iodo-2-(4-methoxyphenyl)chromone (6). Light yellow solid: mp $149-151{ }^{\circ} \mathrm{C}$; ${ }^{1} \mathrm{H}$ NMR (400 MHz, $\left.\mathrm{CDCl}_{3}\right) \delta 3.89$ (s, 3H), 7.00-7.03 (m, 2H), 7.42-7.48 (m, 2H), 7.68$7.81(\mathrm{~m}, 3 \mathrm{H}), 8.25(\mathrm{dd}, J=8.0,2.0 \mathrm{~Hz}, 1 \mathrm{H}) ;{ }^{13} \mathrm{C} \mathrm{NMR}\left(75 \mathrm{MHz}, \mathrm{CDCl}_{3}\right) \delta 55.75$, $87.86,113.79,117.78,120.13,125.93,126.91,127.33,131.61,134.30,155.99,161.86$, 164.49, 174.81; IR ( $\left.\mathrm{CDCl}_{3}, \mathrm{~cm}^{-1}\right)$ 3008, 2939, 1643, 1614; MS (EI) m/z (rel intensity) $378\left(\mathrm{M}^{+}, 100\right), 251$ (16); HRMS (EI) calcd for $\mathrm{C}_{16} \mathrm{H}_{11} \mathrm{IO}_{3} 377.9753$, found: 377.9758. 


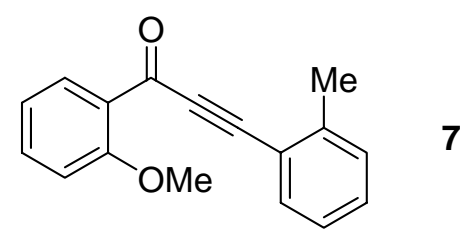

1-(2-Methoxyphenyl)-3-(2-methylphenyl)propynone (7). White solid, obtained in a $72 \%$ yield by Method A using $o$-anisoyl chloride and 2-ethynyltoluene: ${ }^{1} \mathrm{H}$ NMR (400 $\left.\mathrm{MHz}, \mathrm{CDCl}_{3}\right) \delta 2.40(\mathrm{~s}, 3 \mathrm{H}), 3.79(\mathrm{~s}, 3 \mathrm{H}), 6.86-6.92(\mathrm{~m}, 2 \mathrm{H}), 7.06-7.20(\mathrm{~m}, 3 \mathrm{H}), 7.47$ $(\mathrm{m}, 2 \mathrm{H}), 7.98(\mathrm{dd}, J=8.0,2.0 \mathrm{~Hz}, 1 \mathrm{H}) ;{ }^{13} \mathrm{C} \mathrm{NMR}\left(100 \mathrm{MHz}, \mathrm{CDCl}_{3}\right) \delta 20.80,55.95$, $90.80,93.35,112.35,120.46,120.53,126.06,129.98,130.74,132.50,133.65,135.20$, $142.10,159.82,177.64$.

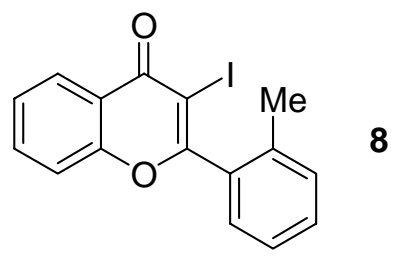

3-Iodo-2-(2-methylphenyl)chromone (8). Light yellow solid: mp $118-121{ }^{\circ} \mathrm{C} ;{ }^{1} \mathrm{H}$ NMR (400 MHz, $\left.\mathrm{CDCl}_{3}\right) \delta 2.30(\mathrm{~s}, 3 \mathrm{H}), 7.34-7.49$ (m, 6H), 7.69-7.72 (m, 1H), 8.29 (dd, $J=8.0,2.0 \mathrm{~Hz}, 1 \mathrm{H}) ;{ }^{13} \mathrm{C}$ NMR $\left(100 \mathrm{MHz}, \mathrm{CDCl}_{3}\right) \delta 19.59,90.48,117.99,120.44$, $126.15,126.21,126.89,129.22,130.79,130.91,134.50,135.64,136.23,156.28,166.42$, 174.49; IR ( $\left.\mathrm{CDCl}_{3}, \mathrm{~cm}^{-1}\right)$ 3008, 2966, 1638; MS (EI) m/z (rel intensity) $362\left(\mathrm{M}^{+}, 100\right)$, 235 (25); HRMS (EI) calcd for $\mathrm{C}_{16} \mathrm{H}_{11} \mathrm{IO}_{2} 361.9804$, found: 361.9809.

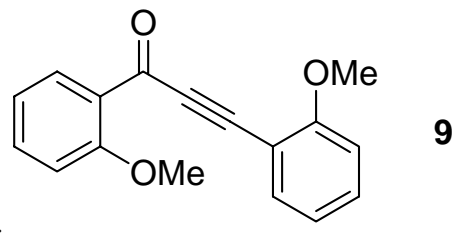

1,3-Di-(2-methoxyphenyl)propynone (9). White solid, obtained in a $75 \%$ yield by Method A using o-anisoyl chloride and 2-ethynylanisole: ${ }^{1} \mathrm{H}$ NMR $\left(400 \mathrm{MHz}, \mathrm{CDCl}_{3}\right) \delta$ 3.82 (s, 3H), 3.87 (s, 3H), 6.82-7.00 (m, 4H), 7.30-7.50 (m, 3H), 8.16 (dd, $J=8.0,2.0$ $\mathrm{Hz}, 1 \mathrm{H}) ;{ }^{13} \mathrm{C}$ NMR $\left(100 \mathrm{MHz}, \mathrm{CDCl}_{3}\right) \delta 56.05,56.09,89.03,93.51,109.88,111.09$, $112.42,120.42,120.82,126.88,132.59,133.39,135.12$, 135.21, 160.05, 161.75, 176.96. 
<smiles>COc1ccccc1-c1oc2ccccc2c(=O)c1I</smiles>

3-Iodo-2-(2-methoxyphenyl)chromone (10). Light yellow solid: mp 133-135 ${ }^{\circ} \mathrm{C} ;{ }^{1} \mathrm{H}$ NMR (400 MHz, $\left.\mathrm{CDCl}_{3}\right) \delta 3.85(\mathrm{~s}, 3 \mathrm{H}), 7.02-7.10(\mathrm{~m}, 2 \mathrm{H}), 7.37-7.69$ (m, 5H), 8.28 $(\mathrm{dd}, J=8.0,2.0 \mathrm{~Hz}, 1 \mathrm{H}) ;{ }^{13} \mathrm{C} \mathrm{NMR}\left(100 \mathrm{MHz}, \mathrm{CDCl}_{3}\right) \delta 55.96,82.29,91.40,111.80$, $118.05,120.49,120.71,125.88,126.84,130.38$, 132.56, 134.22, 156.50, 156.79, 164.45, 174.64; IR $\left(\mathrm{CDCl}_{3}, \mathrm{~cm}^{-1}\right)$ 3007, 2939, 2839, 1643; MS (EI) m/z (rel intensity) $378\left(\mathrm{M}^{+}\right.$, 100), 251 (12); HRMS (EI) calcd for $\mathrm{C}_{16} \mathrm{H}_{11} \mathrm{IO}_{3}$ 377.9753, found: 377.9758.<smiles>CCCCCCCC(=O)c1ccccc1OC(C)=O</smiles>

3-(2-Acetoxyphenyl)-1-(2-methoxyphenyl)propynone (11). White solid, obtained in an $81 \%$ yield by Method A using 0 -anisoyl chloride and 2-ethynylphenyl acetate: ${ }^{1} \mathrm{H}$ NMR (300 MHz, $\left.\mathrm{CDCl}_{3}\right) \delta 2.28(\mathrm{~s}, 3 \mathrm{H}), 3.87(\mathrm{~s}, 3 \mathrm{H}), 6.94-7.25(\mathrm{~m}, 4 \mathrm{H}), 7.41-7.61(\mathrm{~m}$, $3 \mathrm{H}), 8.05(\mathrm{dd}, J=8.0,2.0 \mathrm{~Hz}, 1 \mathrm{H}) ;{ }^{13} \mathrm{C} \mathrm{NMR}\left(75 \mathrm{MHz}, \mathrm{CDCl}_{3}\right) \delta 20.97,56.12,86.38$, $93.47,112.51,115.23,120.46,122.94,126.35,126.62,132.04,133.04,134.33,135.48$, $153.14,160.09,169.11,176.49$.

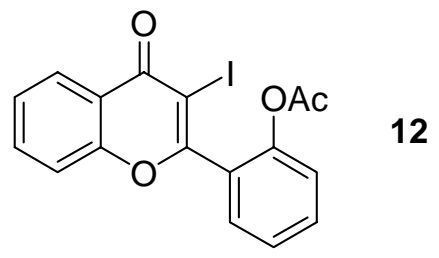

2-(2-Acetoxyphenyl)-3-iodochromone (12). Light yellow oil: ${ }^{1} \mathrm{H}$ NMR $(400 \mathrm{MHz}$, $\left.\mathrm{CDCl}_{3}\right) \delta 2.08(\mathrm{~s}, 3 \mathrm{H}), 7.22-7.45(\mathrm{~m}, 4 \mathrm{H}), 7.51-7.68(\mathrm{~m}, 3 \mathrm{H}), 8.22(\mathrm{~d}, J=8.0 \mathrm{~Hz}, 1 \mathrm{H})$; ${ }^{13} \mathrm{C}$ NMR $\left(100 \mathrm{MHz}, \mathrm{CDCl}_{3}\right) \delta 21.25,90.71,117.84,120.16,123.54,126.17,126.26$, $126.85,128.56,130.99,132.33,134.73,147.98,156.18,162.70,168.79$, 174.33; IR $\left(\mathrm{CDCl}_{3}, \mathrm{~cm}^{-1}\right)$ 3008, 2955, 2850, 1730, 1645; MS (EI) m/z (rel intensity) $406\left(\mathrm{M}^{+}, 100\right)$, 279 (8); HRMS (EI) calcd for $\mathrm{C}_{17} \mathrm{H}_{11} \mathrm{IO}_{4} 405.9702$, found: 405.9708. 


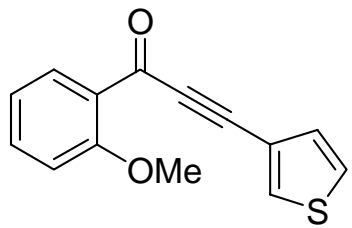

1-(2-Methoxyphenyl)-3-(3-thiophenyl)propynone (13). White solid, obtained in an $80 \%$ yield by Method A using 0 -anisoyl chloride and 3-ethynylthiophene: ${ }^{1} \mathrm{H}$ NMR $\left(400 \mathrm{MHz}, \mathrm{CDCl}_{3}\right) \delta 3.90(\mathrm{~s}, 3 \mathrm{H}), 6.95-7.02(\mathrm{~m}, 2 \mathrm{H}), 7.20-7.30(\mathrm{~m}, 2 \mathrm{H}), 7.45-7.51(\mathrm{~m}$, $1 \mathrm{H}), 7.71-7.73(\mathrm{~m}, 1 \mathrm{H}), 8.02(\mathrm{dd}, J=8.0,2.0 \mathrm{~Hz}, 1 \mathrm{H}) ;{ }^{13} \mathrm{C} \mathrm{NMR}\left(100 \mathrm{MHz}, \mathrm{CDCl}_{3}\right) \delta$ $56.14,87.29,89.65,112.47,120.10,120.54,126.41,126.81,130.46,132.74,133.72$, $135.30,159.98,176.85$.

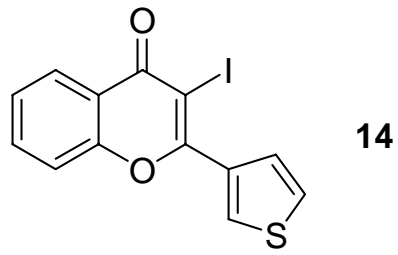

3-Iodo-2-(3-thiophenyl)chromone (14). Brown solid (m.p. 103-106 $\left.{ }^{\circ} \mathrm{C}\right):{ }^{1} \mathrm{H}$ NMR (400 $\left.\mathrm{MHz}, \mathrm{CDCl}_{3}\right) \delta$ 7.39-7.49 (m, 3H), 7.66-7.77 (m, $\left.2 \mathrm{H}\right), 8.22(\mathrm{dd}, J=8.0,1.6 \mathrm{~Hz}, 1 \mathrm{H})$, 8.30-8.32 (m, $1 \mathrm{H}) ;{ }^{13} \mathrm{C}$ NMR $\left(100 \mathrm{MHz}, \mathrm{CDCl}_{3}\right) \delta 87.63,117.71,120.01,125.88$, 125.99, 126.92, 128.48, 131.30, 134.41, 135.29, 155.72, 159.65, 174.70; IR $\left(\mathrm{CDCl}_{3}\right.$, $\mathrm{cm}^{-1}$ ) 3008, 1638; MS (EI) m/z (rel intensity) $354\left(\mathrm{M}^{+}, 75\right), 353$ (100), 135(40); HRMS (EI) calcd for $\mathrm{C}_{13} \mathrm{H}_{7} \mathrm{IO}_{2} \mathrm{~S} 353.9211$, found: 353.9217 .

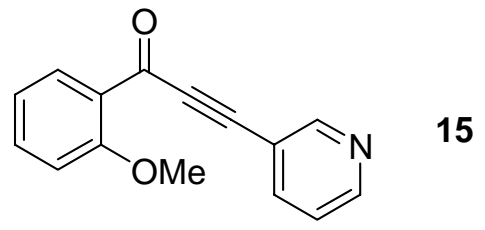

1-(2-Methoxyphenyl)-3-(3-pyridyl)propynone (15). White solid, obtained in an 86\% yield by Method A using $o$-anisoyl chloride and 3-ethynylpyridine: ${ }^{1} \mathrm{H}$ NMR $(400 \mathrm{MHz}$, $\left.\mathrm{CDCl}_{3}\right) \delta 3.78(\mathrm{~s}, 3 \mathrm{H}), 6.84-6.90(\mathrm{~m}, 2 \mathrm{H}), 7.16-7.20(\mathrm{~m}, 1 \mathrm{H}), 7.35-7.38(\mathrm{~m}, 1 \mathrm{H})$, 7.71$7.74(\mathrm{~m}, 1 \mathrm{H}), 7.88$ (dd, $J=7.6,1.6 \mathrm{~Hz}, 1 \mathrm{H}), 8.48(\mathrm{dd}, J=8.8,1.6 \mathrm{~Hz}, 1 \mathrm{H}), 8.66$ (d, $J=$ $1.2 \mathrm{~Hz}, 1 \mathrm{H}) ;{ }^{13} \mathrm{C} \mathrm{NMR}\left(100 \mathrm{MHz}, \mathrm{CDCl}_{3}\right) \delta 56.02,87.64,91.90,112.42,118.08$, $120.54,123.48,126.24,132.52,135.66,139.88,150.60,153.24,160.03,176.06$. 


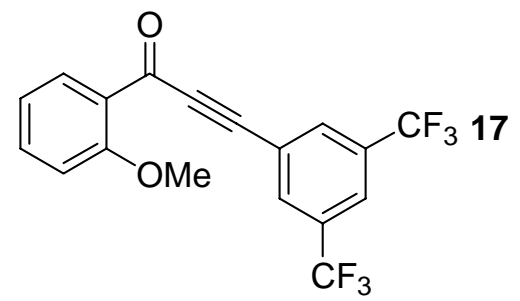

1-(2-Methoxyphenyl)-3-[3,5-bis(trifluoromethyl)phenyl]propynone (17). Yellow solid, obtained in an $85 \%$ yield by Method A using o-anisoyl chloride and 1-ethynyl3,5-bis(trifluoromethyl)benzene: ${ }^{1} \mathrm{H}$ NMR $\left(400 \mathrm{MHz}, \mathrm{CDCl}_{3}\right.$ ) $\delta 3.96$ (s, 3H), 7.02-7.06 (m, 2H), 7.56-7.57 (m, 1H), $7.91(\mathrm{~s}, 1 \mathrm{H}), 8.02-8.06(\mathrm{~m}, 3 \mathrm{H}) ;{ }^{13} \mathrm{C} \mathrm{NMR}(100 \mathrm{MHz}$, $\left.\mathrm{CDCl}_{3}\right) \delta 56.07,86.75,91.15,112.46,115.57,118.83,120.69,121.54,123.55,123.68$, $123.71,123.75,123.78,124.26,126.15,126.97,132.02,132.36,132.66,132.70,132.73$, $133.04,135.93,160.28,175.80$.<smiles>O=c1c(I)c(-c2cc(C(F)(F)F)cc(C(F)(F)F)c2)oc2ccccc12</smiles>

3-Iodo-2-[3,5-bis(trifluoromethyl)phenyl]chromone (18). Yellow solid: ${ }^{1} \mathrm{H}$ NMR $\left(400 \mathrm{MHz}, \mathrm{CDCl}_{3}\right) \delta$ 7.51-7.55 (m, 2H), 7.75-7.78 (m, $\left.1 \mathrm{H}\right), 8.06(\mathrm{~s}, 1 \mathrm{H}), 8.28-8.31(\mathrm{~m}$, $3 \mathrm{H}) ;{ }^{13} \mathrm{C}$ NMR $\left(100 \mathrm{MHz}, \mathrm{CDCl}_{3}\right) \delta 90.04,117.88,120.08,121.69,124.78,124.82$, 124.86, 126.67, 127.14, 130.22, 132.07, 132.40, 134.98, 137.03, 155.99, 161.05, 174.14; IR $\left(\mathrm{CDCl}_{3}, \mathrm{~cm}^{-1}\right)$ 3008, 1638; MS (EI) m/z (rel intensity) $484\left(\mathrm{M}^{+}, 100\right), 357$ (22); HRMS (EI) calcd for $\mathrm{C}_{17} \mathrm{H}_{7} \mathrm{~F}_{6} \mathrm{IO}_{2} 483.9395$, found: 483.9400 .

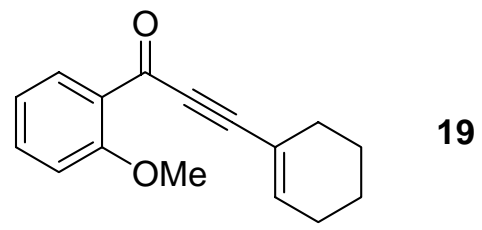

3-(1-Cyclohexenyl)-1-(2-methoxyphenyl)propynone (19). Yellow solid, obtained in an $88 \%$ yield by Method A using o-anisoyl chloride and 1-ethynylcyclohexene: ${ }^{1} \mathrm{H}$ NMR (400 MHz, $\left.\mathrm{CDCl}_{3}\right) \delta$ 1.52-1.62 (m, 4H), 2.07-2.17 (m, 4H), $3.84(\mathrm{~s}, 3 \mathrm{H}), 6.42(\mathrm{~d}$, $J=1.6 \mathrm{~Hz}, 1 \mathrm{H}), 6.89-6.96(\mathrm{~m}, 2 \mathrm{H}), 7.41-7.43(\mathrm{~m}, 1 \mathrm{H}), 7.92(\mathrm{dd}, J=8.0,1.6 \mathrm{~Hz}, 1 \mathrm{H})$; 
${ }^{13} \mathrm{C}$ NMR (100 MHz, $\left.\mathrm{CDCl}_{3}\right) \delta 21.36,22.20,26.36,28.64,56.02,87.80,94.34,112.37$, $119.63,120.37,127.08,132.61,134.93,142.17,159.79,177.06$.

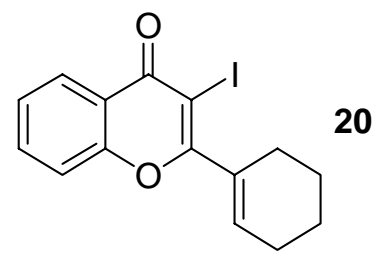

2-(1-Cyclohexenyl)-3-iodochromone (20). Light yellow oil: ${ }^{1} \mathrm{H}$ NMR (400 MHz, $\left.\mathrm{CDCl}_{3}\right) \delta$ 1.68-1.82 (m, 4H), 2.15-2.39 (m, 4H), 6.29-6.32 (m, 1H), 7.36-7.44 (m, 2H), 7.63-7.68 (m, 1H), 8.18-8.21 (m, 1H); ${ }^{13} \mathrm{C}$ NMR $\left(100 \mathrm{MHz}, \mathrm{CDCl}_{3}\right) \delta 21.64,22.39$, $25.28,26.34,87.02,117.72,120.35,125.73,126.78,134.15,134.20,136.05,155.81$, 167.29, 174.93; IR $\left(\mathrm{CDCl}_{3}, \mathrm{~cm}^{-1}\right) 3050,3009,1643,1614 ; \mathrm{MS}$ (EI) m/z (rel intensity) $352\left(\mathrm{M}^{+}, 100\right), 225$ (11); HRMS (EI) calcd for $\mathrm{C}_{15} \mathrm{H}_{13} \mathrm{IO}_{2} 351.9960$, found: 351.9966.

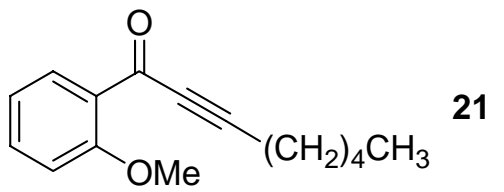

1-(2-Methoxyphenyl)-2-octyn-1-one (21). Colorless oil, obtained in an $82 \%$ yield by Method A using $o$-anisoyl chloride and 1-heptyne: ${ }^{1} \mathrm{H}$ NMR $\left(300 \mathrm{MHz}, \mathrm{CDCl}_{3}\right) \delta 0.84$ (t, $J=7.2 \mathrm{~Hz}, 3 \mathrm{H}), 1.25-1.58$ (m, 6H), 2.35 (t, $J=7.2 \mathrm{~Hz}, 2 \mathrm{H}), 3.81$ (s, 3H), 6.88-6.96 $(\mathrm{m}, 2 \mathrm{H}), 7.38-7.42(\mathrm{~m}, 1 \mathrm{H}), 7.92(\mathrm{dd}, J=7.8,1.8 \mathrm{~Hz}, 1 \mathrm{H}) ;{ }^{13} \mathrm{C} \mathrm{NMR}\left(75 \mathrm{MHz}, \mathrm{CDCl}_{3}\right)$ $\delta 14.09,19.37,22.31,27.72,31.22,55.98,82.00,95.46,112.32,120.30,126.97,132.98$, $134.91,159.80,177.23$.

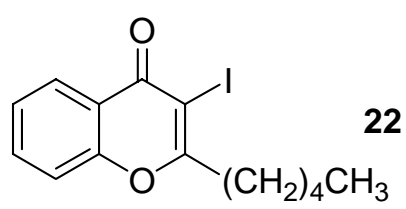

3-Iodo-2-(n-pentyl)chromone (22). Light yellow oil: ${ }^{1} \mathrm{H}$ NMR $\left(400 \mathrm{MHz}, \mathrm{CDCl}_{3}\right) \delta$ $0.93(\mathrm{t}, J=6.8 \mathrm{~Hz}, 3 \mathrm{H}), 1.40-1.81(\mathrm{~m}, 6 \mathrm{H}), 3.03(\mathrm{dt}, J=7.6,0.8 \mathrm{~Hz}, 2 \mathrm{H}), 7.26-7.46(\mathrm{~m}$, $2 \mathrm{H}), 7.67-7.69(\mathrm{~m}, 1 \mathrm{H}), 8.22(\mathrm{~d}, J=8.0 \mathrm{~Hz}, 1 \mathrm{H}) ;{ }^{13} \mathrm{C} \mathrm{NMR}\left(100 \mathrm{MHz}, \mathrm{CDCl}_{3}\right) \delta 14.20$, $22.60,26.90,31.50,38.90,88.57,117.62,120.26,125.77,126.75,134.08,155.77$, 169.52, 174.17; IR $\left(\mathrm{CDCl}_{3}, \mathrm{~cm}^{-1}\right)$ 3007, 2939, 1639; MS (EI) m/z (rel intensity) 342 $\left(\mathrm{M}^{+}, 100\right), 293$ (12); HRMS (EI) calcd for $\mathrm{C}_{14} \mathrm{H}_{15} \mathrm{IO}_{2} 342.0117$, found: 342.0122. 


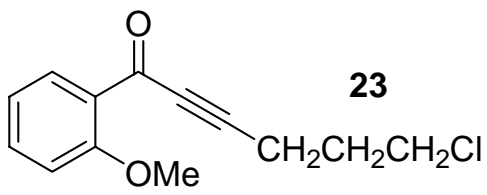

6-Chloro-1-(2-methoxyphenyl)-2-hexyn-1-one (23). Colorless oil, obtained in an 80\% yield by Method A using o-anisoyl chloride and 5-chloro-1-pentyne: ${ }^{1} \mathrm{H}$ NMR (300 $\left.\mathrm{MHz} \mathrm{CDCl}_{3}\right) \delta 1.96-2.03(\mathrm{~m}, 2 \mathrm{H}), 2.59(\mathrm{t}, J=6.9 \mathrm{~Hz}, 2 \mathrm{H}), 3.63(\mathrm{t}, J=6.3 \mathrm{~Hz}, 2 \mathrm{H})$, $3.84(\mathrm{~s}, 3 \mathrm{H}), 6.90-6.97(\mathrm{~m}, 2 \mathrm{H}), 7.41-7.45(\mathrm{~m}, 1 \mathrm{H}), 7.90(\mathrm{dd}, J=7.8,1.8 \mathrm{~Hz}, 1 \mathrm{H}) ;{ }^{13} \mathrm{C}$ NMR $\left(75 \mathrm{MHz}, \mathrm{CDCl}_{3}\right) \delta 16.85,30.72,43.64,56.06,82.62,92.93,112.38,120.43$, $126.75,132.82,135.18,159.86,176.95$.

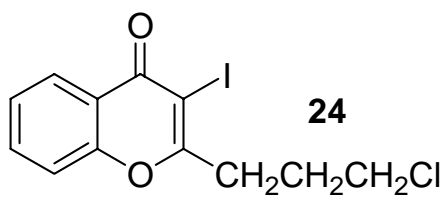

2-(3-Chloropropyl)-3-iodochromone (24). Light yellow solid: mp 75-78 ${ }^{\circ} \mathrm{C} ;{ }^{1} \mathrm{H}$ NMR $\left(400 \mathrm{MHz}, \mathrm{CDCl}_{3}\right) \delta$ 2.24-2.32 (m, 2H), $3.22(\mathrm{t}, J=7.2 \mathrm{~Hz}, 2 \mathrm{H}), 3.67(\mathrm{t}, J=6.4 \mathrm{~Hz}, 2 \mathrm{H})$ 7.39-7.46 (m, 2H), 7.67-7.71 (m, 1H), 8.21 (dd, $J=8.0,1.0 \mathrm{~Hz}, 1 \mathrm{H}) ;{ }^{13} \mathrm{C}$ NMR $(100$ $\left.\mathrm{MHz}, \mathrm{CDCl}_{3}\right) \delta 29.95,36.52,44.04,89.22,117.61,120.26,126.00,126.84,134.26$, 155.73, 167.62, 173.97; IR ( $\left.\mathrm{CDCl}_{3}, \mathrm{~cm}^{-1}\right) 3007,2839,1645 ; \mathrm{MS}$ (EI) m/z (rel intensity) $348\left(\mathrm{M}^{+}, 100\right), 185$ (11); HRMS (EI) calcd for $\mathrm{C}_{12} \mathrm{H}_{10} \mathrm{ClIO}_{2}$ 347.9414, found: 347.9419 .

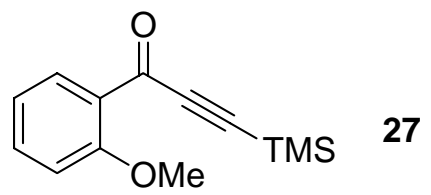

1-(2-Methoxyphenyl)-3-(trimethylsilyl)propynone (27). Colorless oil, obtained in a $95 \%$ yield by the modified Sonogashira method $^{2}$ using $o$-anisoyl chloride and trimethylsilylacetylene: ${ }^{1} \mathrm{H}$ NMR $\left(300 \mathrm{MHz} \mathrm{CDCl}_{3}\right) \delta 0.12(\mathrm{~s}, 9 \mathrm{H}), 3.72(\mathrm{~s}, 3 \mathrm{H}), 6.80$ $6.87(\mathrm{~m}, 2 \mathrm{H}), 7.32-7.36(\mathrm{~m}, 1 \mathrm{H}), 7.82(\mathrm{dd}, J=7.8,1.8 \mathrm{~Hz}, 1 \mathrm{H}) ;{ }^{13} \mathrm{C}$ NMR $(75 \mathrm{MHz}$, $\left.\mathrm{CDCl}_{3}\right) \delta 0.01,56.30,98.80,103.80,112.96,120.92,126.87,133.17,135.90,160.52$, 176.55 . 


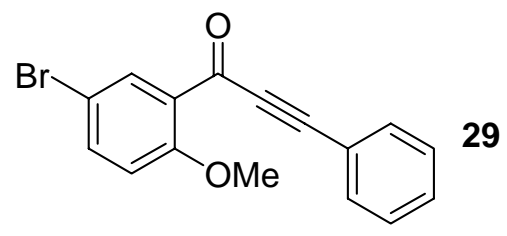

1-(5-Bromo-2-methoxyphenyl)-3-phenylpropynone (29). White solid, obtained in an $86 \%$ overall yield by Method B using 5-bromo-2-methoxybenzaldehyde and lithium phenylacetylide: ${ }^{1} \mathrm{H}$ NMR $\left(300 \mathrm{MHz}, \mathrm{CDCl}_{3}\right) \delta 3.82(\mathrm{~s}, 3 \mathrm{H}), 6.80(\mathrm{~d}, J=9.0 \mathrm{~Hz}, 1 \mathrm{H})$ 7.28-7.52 (m, 6H), 7.97-7.99 (m, 1H); ${ }^{13} \mathrm{C}$ NMR $\left(75 \mathrm{MHz}, \mathrm{CDCl}_{3}\right) \delta$ 56.36, 89.18, $92.79,112.71,114.52,120.45,128.17,128.88,130.97,133.21,134.53,137.65,158.97$, 175.12 .<smiles>O=c1c(I)c(-c2ccccc2)oc2ccc(Br)cc12</smiles>

6-Bromo-3-iodo-2-phenylchromone (30). Light yellow solid: mp $143-146{ }^{\circ} \mathrm{C} ;{ }^{1} \mathrm{H}$ NMR (400 MHz, $\left.\mathrm{CDCl}_{3}\right) \delta$ 7.37-7.40 (m, 1H), 7.52-7.55 (m, 3H), 7.75-7.79 (m, 3H), $8.36(\mathrm{~d}, J=2.4 \mathrm{~Hz}, 1 \mathrm{H}) ;{ }^{13} \mathrm{C} \mathrm{NMR}\left(100 \mathrm{MHz}, \mathrm{CDCl}_{3}\right) \delta 88.33,119.30,119.91,121.32$, $128.59,129.36,129.68,131.50,134.90,137.44,154.84,164.95,173.54$; IR $\left(\mathrm{CDCl}_{3}\right.$, $\mathrm{cm}^{-1}$ ) 3007, 1638; MS (EI) m/z (rel intensity) 427 (42), $425\left(\mathrm{M}^{+}, 63\right), 348$ (100); HRMS (EI) calcd for $\mathrm{C}_{15} \mathrm{H}_{8} \mathrm{BrIO}_{2}$ 425.0752, found: 425.0760 .

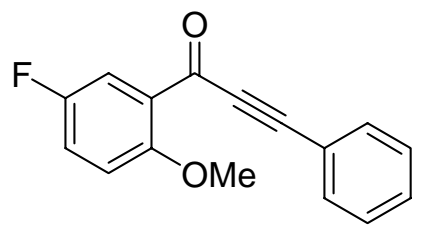

31

1-(5-Fluoro-2-methoxyphenyl)-3-phenylpropynone (31). White solid, obtained in an $88 \%$ overall yield by Method B using 5-fluoro-2-methoxybenzaldehyde and lithium phenylacetylide: ${ }^{1} \mathrm{H}$ NMR $\left(400 \mathrm{MHz}, \mathrm{CDCl}_{3}\right) \delta 3.87(\mathrm{~s}, 3 \mathrm{H}), 6.90-7.66(\mathrm{~m}, 8 \mathrm{H}) ;{ }^{13} \mathrm{C}$ NMR (100 MHz, $\left.\mathrm{CDCl}_{3}\right) \delta 56.71,89.20,92.63,114.01,114.08,118.07,118.31,120.56$, $121.66,121.89,127.48,128.88,130.95,133.24,133.52,155.22,156.30,157.63,175.45$. 
<smiles>O=c1c(I)c(-c2ccccc2)oc2ccc(F)cc12</smiles>

6-Fluoro-3-iodo-2-phenylchromone (32). Light yellow solid: mp $131-133{ }^{\circ} \mathrm{C}$; ${ }^{1} \mathrm{H}$ NMR (400 MHz, $\left.\mathrm{CDCl}_{3}\right) \delta$ 7.41-7.54 (m, 5H), 7.75-7.78 (m, 2H), $7.87(\mathrm{dd}, J=8.0$, $2.4 \mathrm{~Hz}, 1 \mathrm{H}) ;{ }^{13} \mathrm{C} \mathrm{NMR}\left(100 \mathrm{MHz}, \mathrm{CDCl}_{3}\right) \delta 87.72,111.54,111.78,120.12,120.20$, $121.15,121.23,122.67,122.92,128.57,129.66,131.44,135.00,152.35,152.37,158.76$, 161.22, 165.01, 174.12; IR ( $\left.\mathrm{CDCl}_{3}, \mathrm{~cm}^{-1}\right)$ 3007, 1639; $\mathrm{MS}$ (EI) m/z (rel intensity) 366 $\left(\mathrm{M}^{+}, 100\right), 348$ (10), 221 (34); HRMS (EI) calcd for $\mathrm{C}_{15} \mathrm{H}_{8} \mathrm{FIO}_{2}$ 365.9553, found: 365.9558 .

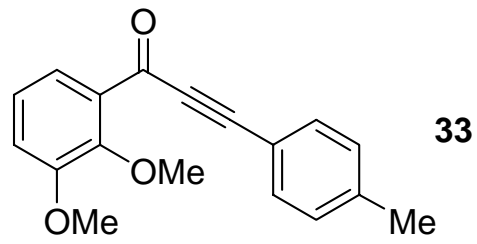

1-(2,3-Dimethoxyphenyl)-3-(4-methylphenyl)propynone (33). White solid, obtained in a 95\% overall yield by Method B using 2,3-dimethoxybenzaldehyde and lithium 4methylphenylacetylide: ${ }^{1} \mathrm{H}$ NMR $\left(400 \mathrm{MHz}, \mathrm{CDCl}_{3}\right) \delta 2.34(\mathrm{~s}, 3 \mathrm{H}), 3.86(\mathrm{~s}, 3 \mathrm{H}), 3.97$ (s, 3H), 7.08-7.17 (m, 4H), 7.49-7.52 (m, 3H); ${ }^{13} \mathrm{C}$ NMR (100 MHz, $\left.\mathrm{CDCl}_{3}\right) \delta$ 21.97, $56.35,61.89,89.69,92.57,117.24,117.60,122.46,124.06,129.67,132.40,133.26$, $141.55,149.93,153.66,177.16$.<smiles>COc1cccc2c(=O)c(I)c(-c3ccc(C)cc3)oc12</smiles>

3-Iodo-2-(4-methylphenyl)-8-methoxychromone (34). Light yellow solid: mp 166$168{ }^{\circ} \mathrm{C} ;{ }^{1} \mathrm{H}$ NMR $\left(400 \mathrm{MHz}, \mathrm{CDCl}_{3}\right) \delta 2.42(\mathrm{~s}, 3 \mathrm{H}), 3.90(\mathrm{~s}, 3 \mathrm{H}), 7.12-7.14(\mathrm{~m}, 1 \mathrm{H})$, 7.27-7.31 (m, 3H), 7.70-7.75 (m, 3H); ${ }^{13} \mathrm{C}$ NMR (100 MHz, $\left.\mathrm{CDCl}_{3}\right) \delta 21.92,56.64$, $88.41,114.76,117.36,121.11,125.70,129.16,129.89,132.24,141.73,146.45,148.71$, 
164.51, 174.66; IR ( $\left.\mathrm{CDCl}_{3}, \mathrm{~cm}^{-1}\right)$ 3007, 2939, 2839, 1642; MS (EI) m/z (rel intensity) $392\left(\mathrm{M}^{+}, 100\right), 265$ (22); HRMS (EI) calcd for $\mathrm{C}_{17} \mathrm{H}_{13} \mathrm{IO}_{3} 391.9909$, found: 391.9915.

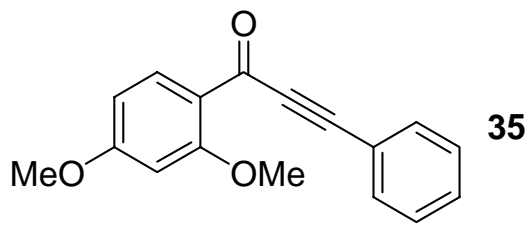

1-(2,4-Dimethoxyphenyl)-3-phenylpropynone (35). White solid, obtained in a $96 \%$ overall yield by Method B using 2,4-dimethoxybenzaldehyde and lithium phenylacetylide; ${ }^{1} \mathrm{H}$ NMR $\left(300 \mathrm{MHz}, \mathrm{CDCl}_{3}\right) \delta 3.56(\mathrm{~s}, 3 \mathrm{H}), 3.63(\mathrm{~s}, 3 \mathrm{H}), 6.19-6.29(\mathrm{~m}$, 2H), 7.09-7.12 (m, 3H), 7.31-7.35 (m, 2H), $7.83(\mathrm{~d}, J=8.7 \mathrm{~Hz}, 1 \mathrm{H}) ;{ }^{13} \mathrm{C}$ NMR $(75$ $\left.\mathrm{MHz}, \mathrm{CDCl}_{3}\right) \delta 55.60,55.76,89.38,90.61,98.46,105.58,119.87,120.82,128.70$, $130.38,132.80,135.03,162.12,165.75,174.64$.

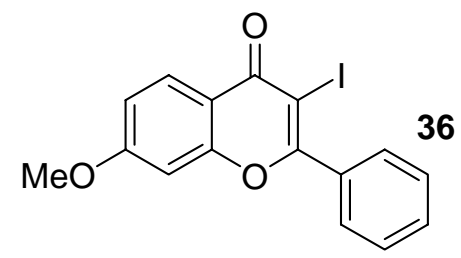

3-Iodo-7-methoxy-2-phenylchromone (36). Light yellow solid: mp $173-175{ }^{\circ} \mathrm{C} ;{ }^{1} \mathrm{H}$ NMR (400 MHz, $\left.\mathrm{CDCl}_{3}\right) \delta 3.86(\mathrm{~s}, 3 \mathrm{H}), 6.82(\mathrm{~s}, 1 \mathrm{H}), 6.94-6.97(\mathrm{~m}, 1 \mathrm{H})$, 7.50-7.74 (m, $5 \mathrm{H}), 8.11(\mathrm{~d}, J=8.8 \mathrm{~Hz}, 1 \mathrm{H}) ;{ }^{13} \mathrm{C}$ NMR $\left(100 \mathrm{MHz}, \mathrm{CDCl}_{3}\right) \delta 56.22,88.87,99.86$, 114.04, 115.58, 128.27, 128.78, 129.66, 131.17, 135.27, 157.81, 164.21, 164.63, 173.89; IR $\left(\mathrm{CDCl}_{3}, \mathrm{~cm}^{-1}\right)$ 3007, 1623; MS (EI) m/z (rel intensity) $378\left(\mathrm{M}^{+}, 100\right), 251$ (22); HRMS (EI) calcd for $\mathrm{C}_{16} \mathrm{H}_{11} \mathrm{IO}_{3} 377.9753$, found: 377.9760 .<smiles>COc1cc(OC)c(C(=O)C#Cc2ccccc2)cc1OC</smiles>

1-(2,4,5-Trimethoxyphenyl)-3-phenylpropynone (37). White solid, obtained in an $88 \%$ overall yield by Method B using 2,4,5-trimethoxybenzaldehyde and lithium phenylacetylide: ${ }^{1} \mathrm{H}$ NMR (300 MHz, $\left.\mathrm{CDCl}_{3}\right) \delta 3.73$ (s, 3H), 3.82 (s, 3H), $3.83(\mathrm{~s}, 3 \mathrm{H})$, $6.39(\mathrm{~s}, 1 \mathrm{H}), 7.23-7.48(\mathrm{~m}, 6 \mathrm{H}) ;{ }^{13} \mathrm{C} \mathrm{NMR}\left(100 \mathrm{MHz}, \mathrm{CDCl}_{3}\right) \delta 56.34,56.37,56.78$, 
$90.02,91.15,97.04,113.38,118.50,121.10,128.77,130.44,132.97,143.01,155.46$, $157.01,174.28$.<smiles>COc1cc2oc(-c3ccccc3)c(I)c(=O)c2cc1OC</smiles>

3-Iodo-6,7-dimethoxy-2-phenylchromone (38). Light yellow solid: $\mathrm{mp} 253-255{ }^{\circ} \mathrm{C}$; ${ }^{1} \mathrm{H}$ NMR (400 MHz, $\mathrm{CDCl}_{3}$ ) $\delta 3.96$ (s, 3H), 3.99 (s, 3H), 6.89 (s, 1H), 7.52-7.77 (m, $6 \mathrm{H}) ;{ }^{13} \mathrm{C}$ NMR $\left(100 \mathrm{MHz}, \mathrm{CDCl}_{3}\right) \delta 56.69,56.77,99.39,105.40,128.48,129.64$, 131.06, 135.43, 148.33, 152.28, 155.06, 164.01, 174.39; IR $\left(\mathrm{CDCl}_{3}, \mathrm{~cm}^{-1}\right)$ 3007, 2939, 2839, 1619; MS (EI) m/z (rel intensity) 408 ( $\left.\mathrm{M}^{+}, 100\right), 281$ (25); HRMS (EI) calcd for $\mathrm{C}_{17} \mathrm{H}_{13} \mathrm{IO}_{4} 407.9859$, found: 407.9866 .<smiles>O=c1c(I)c(-c2ccccc2)oc2cc([N+](=O)[O-])ccc12</smiles>

3-Iodo-7-nitro-2-phenylchromone (42). Yellow solid: mp 163-165 ${ }^{\circ} \mathrm{C}$; ${ }^{1} \mathrm{H}$ NMR (400 $\left.\mathrm{MHz}, \mathrm{CDCl}_{3}\right) \delta$ 7.57-7.82 (m, 6H), 8.53-8.55 (m, 1H), 9.09-9.12 (m, $\left.1 \mathrm{H}\right) ;{ }^{13} \mathrm{C}$ NMR $\left(100 \mathrm{MHz}, \mathrm{CDCl}_{3}\right) \delta 88.77,119.80,120.05,123.69,128.72,129.05,129.71,131.89$, 134.28, 145.37, 158.80, 165.19, 173.53; IR $\left(\mathrm{CDCl}_{3}, \mathrm{~cm}^{-1}\right) 3007,1657,1625 ; \mathrm{MS}(\mathrm{EI})$ $\mathrm{m} / \mathrm{z}$ (rel intensity) $393\left(\mathrm{M}^{+}, 100\right), 364$ (18); HRMS (EI) calcd for $\mathrm{C}_{15} \mathrm{H}_{8} \mathrm{INO}_{4} 392.9498$, found: 392.9504 .

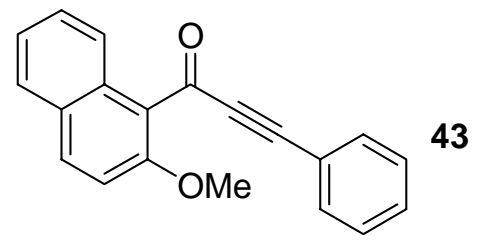

1-(2-Methoxy-1-naphthyl)-3-phenylpropynone (43). White solid, obtained in an 85\% overall yield by Method B using 2-methoxy-1-naphthaldehyde and lithium phenylacetylide: ${ }^{1} \mathrm{H}$ NMR (400 MHz, $\left.\mathrm{CDCl}_{3}\right) \delta 3.99$ (s, 3H), 7.26-7.58 (m, 8H), 7.78 (d, $J=8.0 \mathrm{~Hz}, 1 \mathrm{H}), 7.91(\mathrm{~d}, J=9.2 \mathrm{~Hz}, 1 \mathrm{H}), 8.16(\mathrm{~d}, J=8.8 \mathrm{~Hz}, 1 \mathrm{H}) ;{ }^{13} \mathrm{C} \mathrm{NMR}(100 \mathrm{MHz}$, 
$\left.\mathrm{CDCl}_{3}\right) \delta 57.09,90.90,91.52,113.70,120.69,123.41,124.00,124.64,128.43,128.62$, $128.88,129.04,130.89,131.17,133.30,133.56,156.77,180.62$.

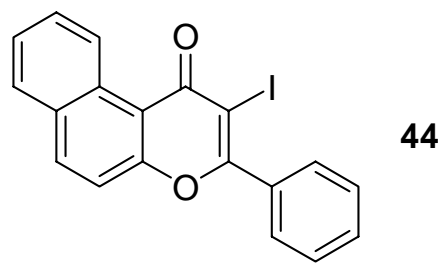

3-Iodo-2-phenylnaphtho[2,1-b]pyran-1-one (44). Yellow solid: mp 182-185 ${ }^{\circ} \mathrm{C} ;{ }^{1} \mathrm{H}$ NMR $\left(300 \mathrm{MHz}, \mathrm{CDCl}_{3}\right) \delta$ 7.44-7.88 (m, 9H), $8.05(\mathrm{~d}, J=9.0 \mathrm{~Hz}, 1 \mathrm{H}), 10.03(\mathrm{~d}, J=$ $9.0 \mathrm{~Hz}, 1 \mathrm{H}) ;{ }^{13} \mathrm{C} \mathrm{NMR}\left(75 \mathrm{MHz}, \mathrm{CDCl}_{3}\right) \delta 93.15,113.51,117.15,127.19,127.48$, 128.56, 129.76, 129.81, 130.21, 130.98, 131.24, 134.90, 136.30, 157.28, 162.64, 175.70; IR $\left(\mathrm{CDCl}_{3}, \mathrm{~cm}^{-1}\right)$ 3010, 1630; MS (EI) m/z (rel intensity) $398\left(\mathrm{M}^{+}, 100\right), 271$ (32); HRMS (EI) calcd for $\mathrm{C}_{19} \mathrm{H}_{11} \mathrm{IO}_{2}$ 397.9804, found: 397.9810 .

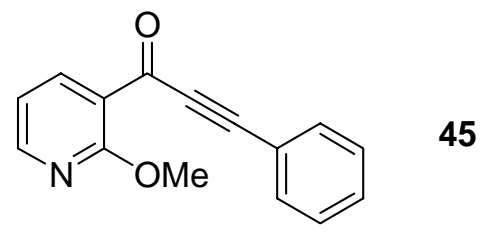

1-(2-Methoxy-3-pyridyl)-3-phenylpropynone (45). White solid, obtained in an $80 \%$ overall yield by Method B using 2-methoxy-3-pyridinecarboxaldehyde and lithium phenylacetylide: ${ }^{1} \mathrm{H}$ NMR (400 MHz, $\left.\mathrm{CDCl}_{3}\right) \delta 3.99$ (s, 3H), 6.90-6.92 (m, 1H) 7.26$7.52(\mathrm{~m}, 5 \mathrm{H}), 8.23-8.27$ (m, 2H); ${ }^{13} \mathrm{C}$ NMR $\left(100 \mathrm{MHz}, \mathrm{CDCl}_{3}\right) \delta 54.25,88.76,92.91$, $116.90,120.39,120.70,128.86,130.98,133.23,141.78,152.10,162.52,175.48$.

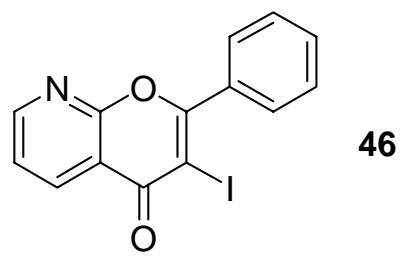

3-Iodo-2-phenyl-4H-pyrano[2,3-b]pyridin-4-one (46). Light yellow solid: mp 131$133{ }^{\circ} \mathrm{C} ;{ }^{1} \mathrm{H}$ NMR $\left(400 \mathrm{MHz}, \mathrm{CDCl}_{3}\right) \delta$ 7.48-7.54 (m, 4H), 7.82-7.85 (m, 2H), 8.63$8.73(\mathrm{~m}, 2 \mathrm{H}) ;{ }^{13} \mathrm{C}$ NMR $\left(100 \mathrm{MHz}, \mathrm{CDCl}_{3}\right) \delta 88.41,114.79,123.04,128.51,129.90$, 
131.62, 134.52, 137.64, 154.08, 160.53, 165.05, 175.43; IR $\left(\mathrm{CDCl}_{3}, \mathrm{~cm}^{-1}\right) 3007,1653$; MS (EI) m/z (rel intensity) $349\left(\mathrm{M}^{+}, 100\right), 222$ (20); HRMS (EI) calcd for $\mathrm{C}_{14} \mathrm{H}_{8} \mathrm{NIO}_{2}$ 348.9600, found: 348.9605 .<smiles>CS(=O)(=O)c1ccccc1C(=O)C#CC1=CCCCC1</smiles>

49

3-(1-Cyclohexenyl)-1-[2-(methylthio)phenyl]propynone (49). White solid, obtained in a $72 \%$ overall yield by Method B using 2-(methylthio)benzaldehyde and lithium 1cyclohexenylacetylide: ${ }^{1} \mathrm{H}$ NMR $\left(300 \mathrm{MHz}, \mathrm{CDCl}_{3}\right) \delta$ 1.50-1.78 (m, 4H), 2.05-2.25 (m, 4H), 2.40 (s, 3H), 6.40-6.45 (m, 1H), 7.12-7.23 (m, 2H), 7.39-7.46 (m, 1H), 8.23 (dd, $J$ $=7.8,1.5 \mathrm{~Hz}, 1 \mathrm{H}) ;{ }^{13} \mathrm{C} \mathrm{NMR}\left(100 \mathrm{MHz}, \mathrm{CDCl}_{3}\right) \delta 15.68,21.33,22.17,26.35,28.57$, $85.71,95.40,119.31,123.37,124.34,133.32,136.66,142.33,144.58,178.02$.<smiles>O=c1c(I)c(C2=CCCCC2)sc2ccccc12</smiles>

2-(1-Cyclohexenyl)-3-iodothiochromenone (50). Light yellow oil: ${ }^{1} \mathrm{H}$ NMR (400 MHz, $\left.\mathrm{CDCl}_{3}\right) \delta$ 1.64-1.82 (m, 4H), 2.21-2.43 (m, 4H), 5.88-5.89 (m, 1H), 7.51-7.61 (m, 3H), $8.54(\mathrm{~d}, J=8.0 \mathrm{~Hz}, 1 \mathrm{H}) ;{ }^{13} \mathrm{C} \mathrm{NMR}\left(100 \mathrm{MHz}, \mathrm{CDCl}_{3}\right) \delta 21.61,22.61,25.44,28.10$, $101.18,123.36,125.40,127.65,128.39,130.10,131.67,131.85,137.97,139.64,157.05$, 176.96; IR $\left(\mathrm{CDCl}_{3}, \mathrm{~cm}^{-1}\right) 3025,3007,1617$; MS (EI) m/z (rel intensity) $368\left(\mathrm{M}^{+}, 100\right)$, 241 (25); HRMS (EI) calcd for $\mathrm{C}_{15} \mathrm{H}_{13} \mathrm{IOS} 367.9732$, found: 367.9736.<smiles>COc1ccccc1C#CC(=O)c1ccccc1C</smiles>

3-(2-Methoxyphenyl)-1-[2-(methylthio)phenyl]propynone (51). White solid, obtained in an $88 \%$ overall yield by Method B using 2-(methylthio)benzaldehyde and lithium 2-methoxyphenylacetylide: ${ }^{1} \mathrm{H}$ NMR (300 MHz, $\left.\mathrm{CDCl}_{3}\right) \delta 2.39$ (s, 3H), 3.87 (s, 
$3 \mathrm{H}), 6.85-6.93(\mathrm{~m}, 2 \mathrm{H}), 7.17-7.54(\mathrm{~m}, 5 \mathrm{H}), 8.57(\mathrm{dd}, J=8.0,1.5 \mathrm{~Hz}, 1 \mathrm{H}) ;{ }^{13} \mathrm{C} \mathrm{NMR}$ $\left(100 \mathrm{MHz}, \mathrm{CDCl}_{3}\right) \delta 15.66,56.14,90.30,91.75,109.58,111.10,120.86,123.48,124.28$, $132.78,133.36,133.46,134.92,135.38,144.77,161.97,177.99$.

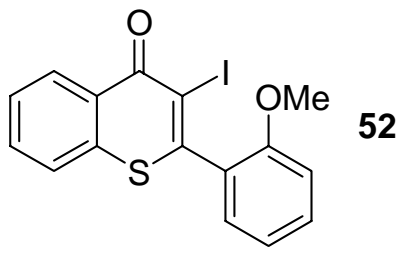

3-Iodo-2-(2-methoxyphenyl)thiochromenone (52). Light yellow oil: ${ }^{1} \mathrm{H}$ NMR (400 $\left.\mathrm{MHz}, \mathrm{CDCl}_{3}\right) \delta 3.77(\mathrm{~s}, 3 \mathrm{H}), 6.93-7.19(\mathrm{~m}, 3 \mathrm{H}), 7.42-7.56(\mathrm{~m}, 4 \mathrm{H}), 8.53(\mathrm{~d}, J=8.8 \mathrm{~Hz}$, $1 \mathrm{H}) ;{ }^{13} \mathrm{C}$ NMR $\left(100 \mathrm{MHz}, \mathrm{CDCl}_{3}\right) \delta 56.03,105.38,111.93,120.97,125.46,127.67$, $128.45,129.78,130.22,131.92,131.96,138.19,152.30,155.77,176.44$; IR $\left(\mathrm{CDCl}_{3}\right.$, $\left.\mathrm{cm}^{-1}\right)$ 3007, 2945, 2839, 1618; MS (EI) m/z (rel intensity) $394\left(\mathrm{M}^{+}, 100\right), 267$ (18); HRMS (EI) calcd for $\mathrm{C}_{16} \mathrm{H}_{11} \mathrm{IO}_{3} 393.9796$, found: 393.9800 .<smiles>COc1cc(C#CC(=O)c2ccccc2C)cc(OC)c1</smiles>

3-(3,5-Dimethoxyphenyl)-1-[2-(methylthio)phenyl]propynone (53). White solid, obtained in a $72 \%$ overall yield by Method B using 2-(methylthio)benzaldehyde and lithium 3,5-dimethoxyphenylacetylide: ${ }^{1} \mathrm{H}$ NMR $\left(300 \mathrm{MHz}, \mathrm{CDCl}_{3}\right) \delta 2.44(\mathrm{~s}, 3 \mathrm{H}), 3.79$ (s, 6H), 6.54-6.56 (m, 1H), $6.78(\mathrm{~d}, J=2.4 \mathrm{~Hz}, 2 \mathrm{H}), 7.22-7.32(\mathrm{~m}, 2 \mathrm{H}), 7.48-7.55(\mathrm{~m}$, $1 \mathrm{H}), 8.38(\mathrm{dd}, J=7.7,1.2 \mathrm{~Hz}, 1 \mathrm{H}) ;{ }^{13} \mathrm{C} \mathrm{NMR}\left(100 \mathrm{MHz}, \mathrm{CDCl}_{3}\right) \delta 15.68,55.78,86.70$, $92.88,104.23,110.74,121.66,123.49,124.45,133.17,133.59$, 134.93, 145.01, 160.87, 177.78 .<smiles>COc1cc(OC)cc(-c2sc3ccccc3c(=O)c2I)c1</smiles> 
2-(3,5-Dimethoxyphenyl)-3-iodothiochromenone (54). Light yellow solid: mp 93-96 ${ }^{\circ} \mathrm{C}$; ${ }^{1} \mathrm{H}$ NMR (400 MHz, $\mathrm{CDCl}_{3}$ ) $\delta 3.81$ (s, 6H), 6.55-6.59 (m, 3H), 7.58-7.66 (m, 3H), $8.60(\mathrm{~d}, J=8.4 \mathrm{~Hz}, 1 \mathrm{H}) ;{ }^{13} \mathrm{C} \mathrm{NMR}\left(100 \mathrm{MHz}, \mathrm{CDCl}_{3}\right) \delta 55.82,102.18,103.00,106.95$, 110.76, 125.36, 127.59, 128.65, 130.28, 132.16, 137.55, 142.47, 154.27, 161.00, 176.50; IR $\left(\mathrm{CDCl}_{3}, \mathrm{~cm}^{-1}\right)$ 3009, 2961, 2938, 2839, 1617; MS (EI) m/z (rel intensity) $424\left(\mathrm{M}^{+}\right.$, 100), 297 (22); HRMS (EI) calcd for $\mathrm{C}_{17} \mathrm{H}_{13} \mathrm{IO}_{3} \mathrm{~S} 423.9630$, found: 423.9630 .

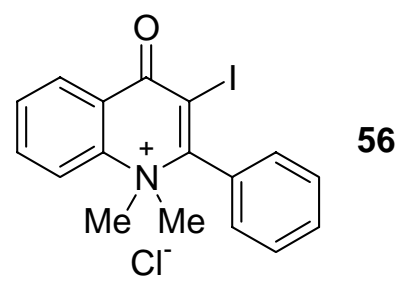

3-Iodo-1,1-dimethyl-4-oxo-2-phenyl-1,4-dihydroquinolinium chloride (56). Light yellow solid: ${ }^{1} \mathrm{H}$ NMR (400 MHz, $\left.\mathrm{CDCl}_{3}\right) \delta 2.83(\mathrm{~s}, 6 \mathrm{H}), 6.96-7.04(\mathrm{~m}, 2 \mathrm{H}), 7.35-7.40$ $(\mathrm{m}, 4 \mathrm{H}), 7.52-7.59(\mathrm{~m}, 2 \mathrm{H}), 7.69-7.74(\mathrm{~m}, 1 \mathrm{H}) ;{ }^{13} \mathrm{C} \mathrm{NMR}\left(100 \mathrm{MHz}, \mathrm{CDCl}_{3}\right) \delta 39.48$, 89.49, 115.96, 123.20, 124.73, 128.11, 128.50, 129.48, 130.00, 132.88, 139.65, 141.00, 155.50, 174.30; IR $\left(\mathrm{CDCl}_{3}, \mathrm{~cm}^{-1}\right)$ 3007, 1630; $\mathrm{MS}(\mathrm{EI}) \mathrm{m} / \mathrm{z}$ (rel intensity) $376\left(\mathrm{M}^{+}, 1\right)$, 361 (100), 234 (35); HRMS (EI) calcd for $\mathrm{C}_{17} \mathrm{H}_{15} \mathrm{INO} 376.0198$, found: 376.0205.

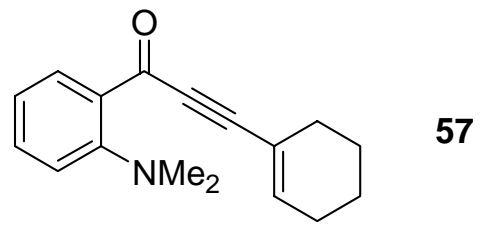

3-(1-Cyclohexenyl)-1-[2-(dimethylamino)phenyl]propynone (57). Colorless oil, obtained in a $66 \%$ overall yield by Method B using 2-(dimethylamino)benzaldehyde and lithium 1-cyclohexenylacetylide: ${ }^{1} \mathrm{H}$ NMR $\left(300 \mathrm{MHz}, \mathrm{CDCl}_{3}\right) \delta 1.55-1.66(\mathrm{~m}, 4 \mathrm{H})$, 2.12-2.20 (m, 4H), $2.86(\mathrm{~s}, 6 \mathrm{H}), 6.43-6.45(\mathrm{~m}, 1 \mathrm{H}), 6.80-6.92(\mathrm{~m}, 2 \mathrm{H}), 7.31-7.34(\mathrm{~m}$, $1 \mathrm{H}), 8.00(\mathrm{dd}, J=8.0,1.6 \mathrm{~Hz}, 1 \mathrm{H}) ;{ }^{13} \mathrm{C} \mathrm{NMR}\left(100 \mathrm{MHz}, \mathrm{CDCl}_{3}\right) \delta 21.42,22.26,26.36$, 28.72, 44.19, 87.19, 93.28, 116.57, 117.97, 119.62, 133.69, 133.90, 141.70, 152.68, 178.20 . 


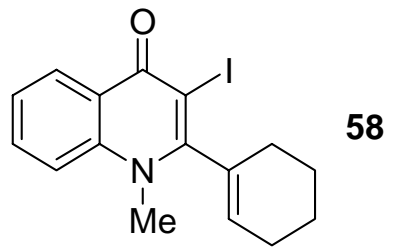

2-(1-Cyclohexenyl)-3-iodo- $N$-methyl-4(1H)-quinolinone (58). Light yellow oil: ${ }^{1} \mathrm{H}$ NMR (400 MHz, $\left.\mathrm{CDCl}_{3}\right) \delta$ 1.60-2.40 (m, 8H), $3.84(\mathrm{~s}, 3 \mathrm{H}), 5.83(\mathrm{~s}, 1 \mathrm{H}), 7.34-7.38(\mathrm{~m}$, 1H), 7.47-7.50 (m, 1H), 7.62-7.67 (m, 1H), $8.45(\mathrm{~d}, J=7.2 \mathrm{~Hz}, 1 \mathrm{H}) ;{ }^{13} \mathrm{C}$ NMR $(100$ $\left.\mathrm{MHz}, \mathrm{CDCl}_{3}\right) \delta 21.60,22.50,25.25,28.01,38.04,88.02,115.77,123.07,124.41$, 127.97, 132.23, 132.55, 137.57, 140.72, 156.90, 174.45; IR $\left(\mathrm{CDCl}_{3}, \mathrm{~cm}^{-1}\right)$ 3007, 2942, 1615; MS (EI) m/z (rel intensity) $365\left(\mathrm{M}^{+}, 100\right), 238$ (32); HRMS (EI) calcd for $\mathrm{C}_{16} \mathrm{H}_{16} \mathrm{INO} 365.0277$, found: 365.0283 .<smiles>COc1ccc2c(=O)c(-c3ccc(C)cc3)c(-c3ccccc3)oc2c1</smiles>

7-Methoxy-3-(4-methylphenyl)-2-phenylchromone (59). White solid: mp $163-166{ }^{\circ} \mathrm{C}$; ${ }^{1} \mathrm{H}$ NMR $\left(300 \mathrm{MHz}, \mathrm{CDCl}_{3}\right) \delta 2.33$ (s, 3H), 3.91 (s, 3H), 6.91-7.00 (m, 2H), 7.05-7.15 (m, 4H), 7.21-7.42 (m, 5H), $8.18(\mathrm{~d}, J=8.7 \mathrm{~Hz}, 1 \mathrm{H}) ;{ }^{13} \mathrm{C} \mathrm{NMR}\left(75 \mathrm{MHz}, \mathrm{CDCl}_{3}\right) \delta$ 21.56, 56.07, 82.29, 100.24, 114.73, 117.62, 128.01, 128.28, 129.24, 129.73, 130.03, 130.08, 131.28, 133.73, 137.43, 158.00, 161.08, 164.34, 177.73; IR $\left(\mathrm{CDCl}_{3}, \mathrm{~cm}^{-1}\right)$ 3007, 2941, 2839, 1639; MS (EI) m/z (rel intensity) $342\left(\mathrm{M}^{+}, 100\right), 329$ (25); HRMS (EI) calcd for $\mathrm{C}_{23} \mathrm{H}_{18} \mathrm{O}_{3} 342.1256$, found: 342.1262 .

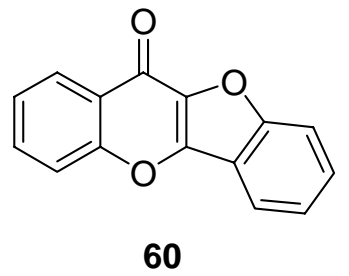

Benzofuro[3,2-b][1]chromone (60). ${ }^{3}$ White solid: ${ }^{1} \mathrm{H} \mathrm{NMR}\left(400 \mathrm{MHz}, \mathrm{CDCl}_{3}\right) \delta$ 7.437.79 (m, 6H), 7.98 (d, $J=8.0 \mathrm{~Hz}, 1 \mathrm{H}), 8.48(\mathrm{dd}, J=8.0,1.2 \mathrm{~Hz}, 1 \mathrm{H}) ;{ }^{13} \mathrm{C}$ NMR $(100$ $\left.\mathrm{MHz}, \mathrm{CDCl}_{3}\right) \delta 113.69,118.26,120.80,124.41,125.11,126.77,128.67,128.80,130.73$, 
$132.43,132.54,133.66,155.31,156.05,176.68 ; \mathrm{MS}(\mathrm{EI}) \mathrm{m} / \mathrm{z}$ (rel intensity) $236\left(\mathrm{M}^{+}\right.$, 100), 208 (23); HRMS (EI) calcd for $\mathrm{C}_{15} \mathrm{H}_{8} \mathrm{O}_{3} 236.0473$, found: 236.0477.

\section{References}

(1) Costa, A. M. B. S. R. C. S.; Dean, F. M.; Jones, M. A.; Varma, R. S. J. Chem. Soc., Perkin Trans. I 1985, 799.

(2) Karpov, A. S.; Mueller, T. J. Org. Lett. 2003, 5, 3451.

(3) Collini, M. D.; Miller, C. P. Tetrahedron Lett. 2001, 42, 8429. 
대 ᄂ 0 는 r.

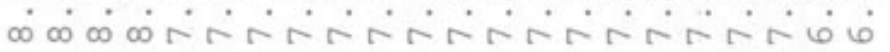

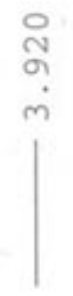<smiles>COc1ccccc1C(=O)C#Cc1ccccc1</smiles>

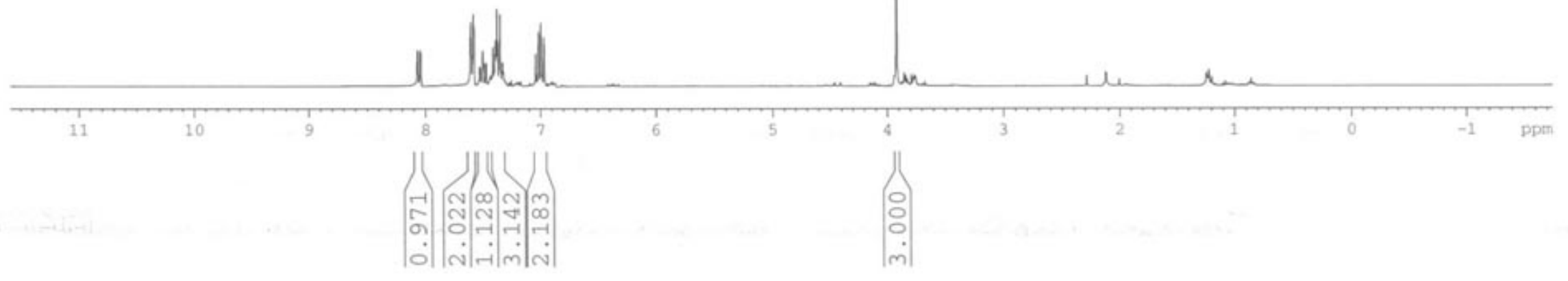

S23 

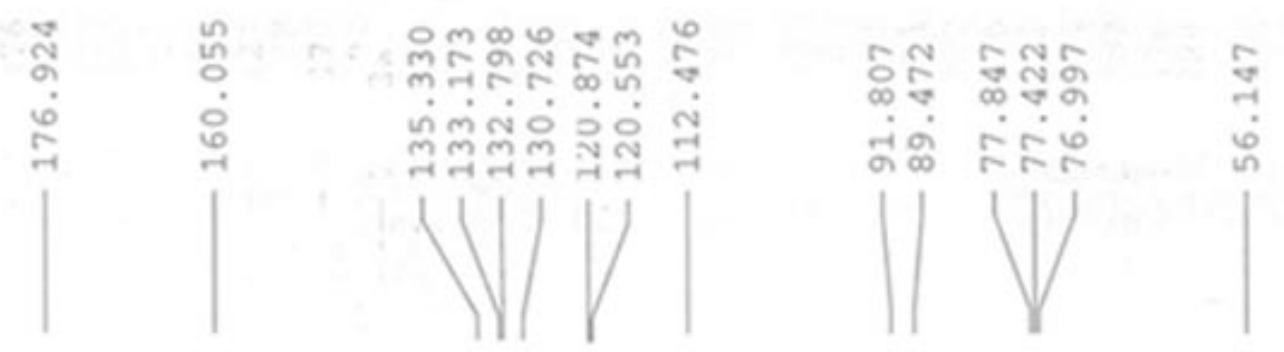<smiles>COc1ccccc1C(=O)C#Cc1ccccc1</smiles>

1

S24 

m कातितनती

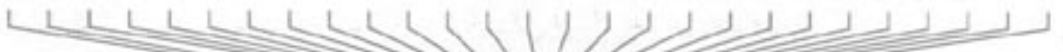
17 II

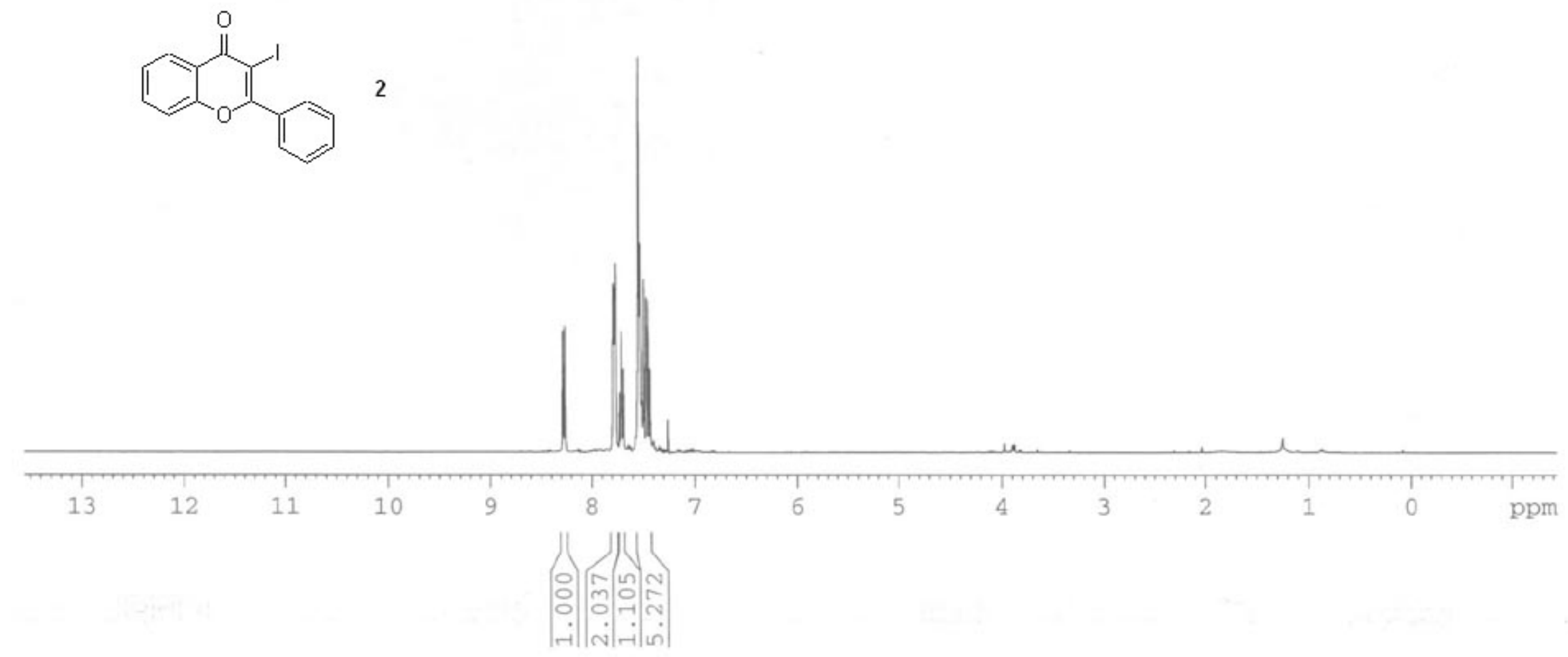

2 


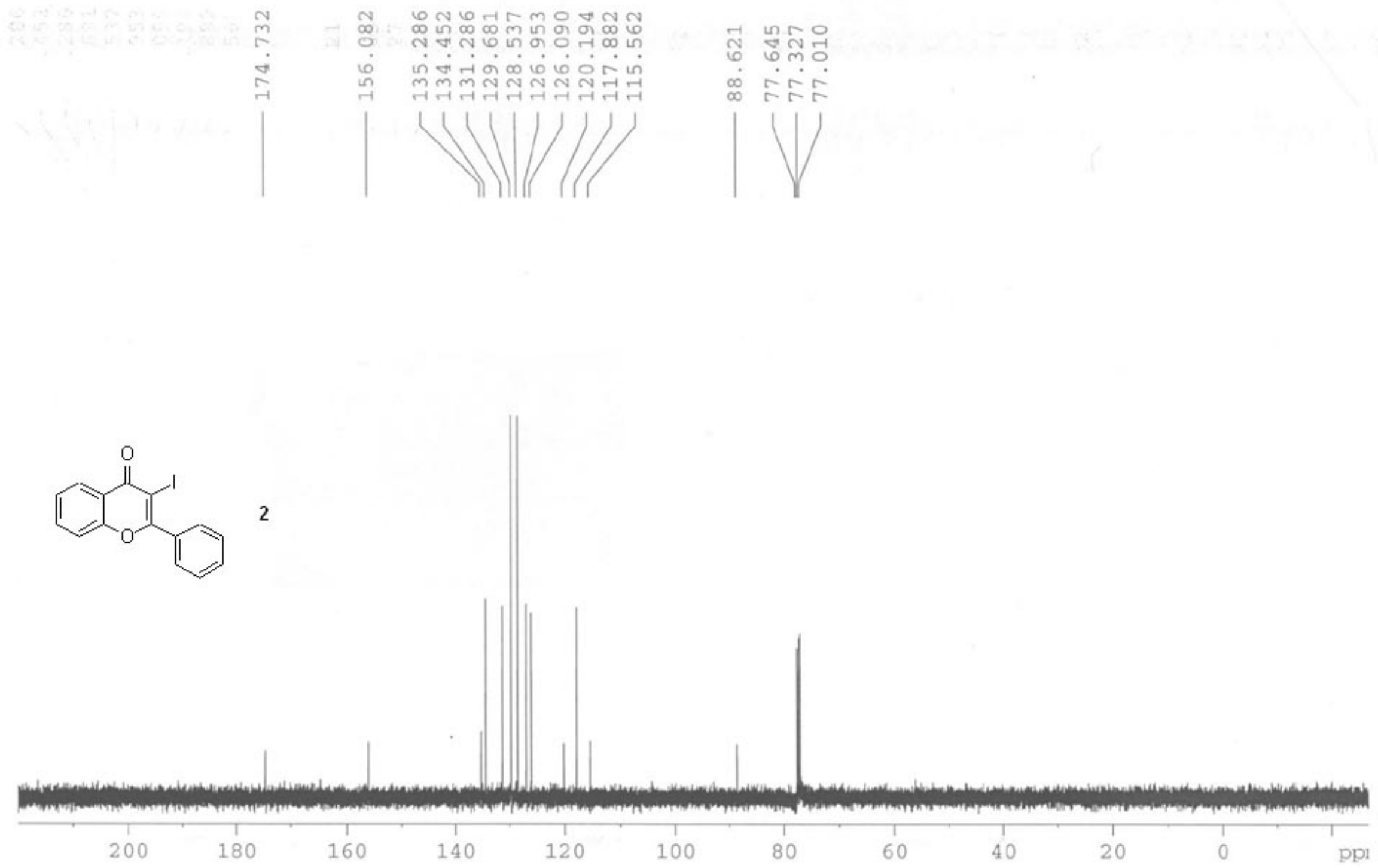




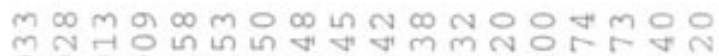

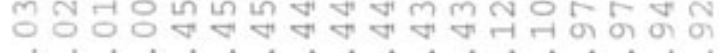

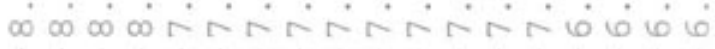

$\longrightarrow \rightarrow+1$

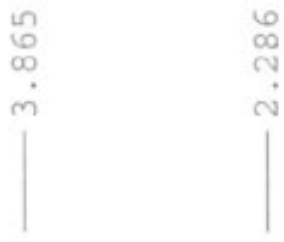

${ }_{\mathrm{OMe}}^{\infty}$

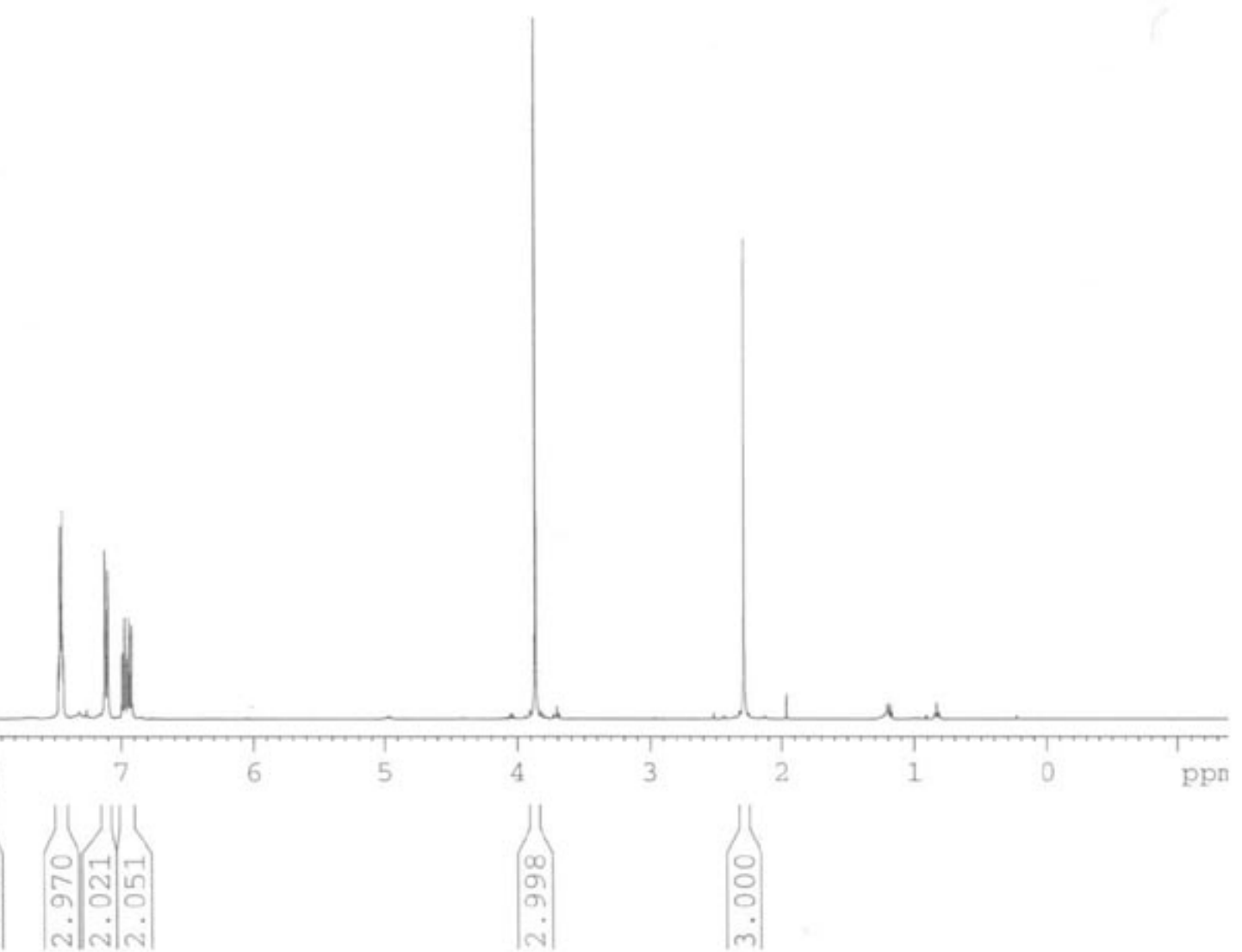




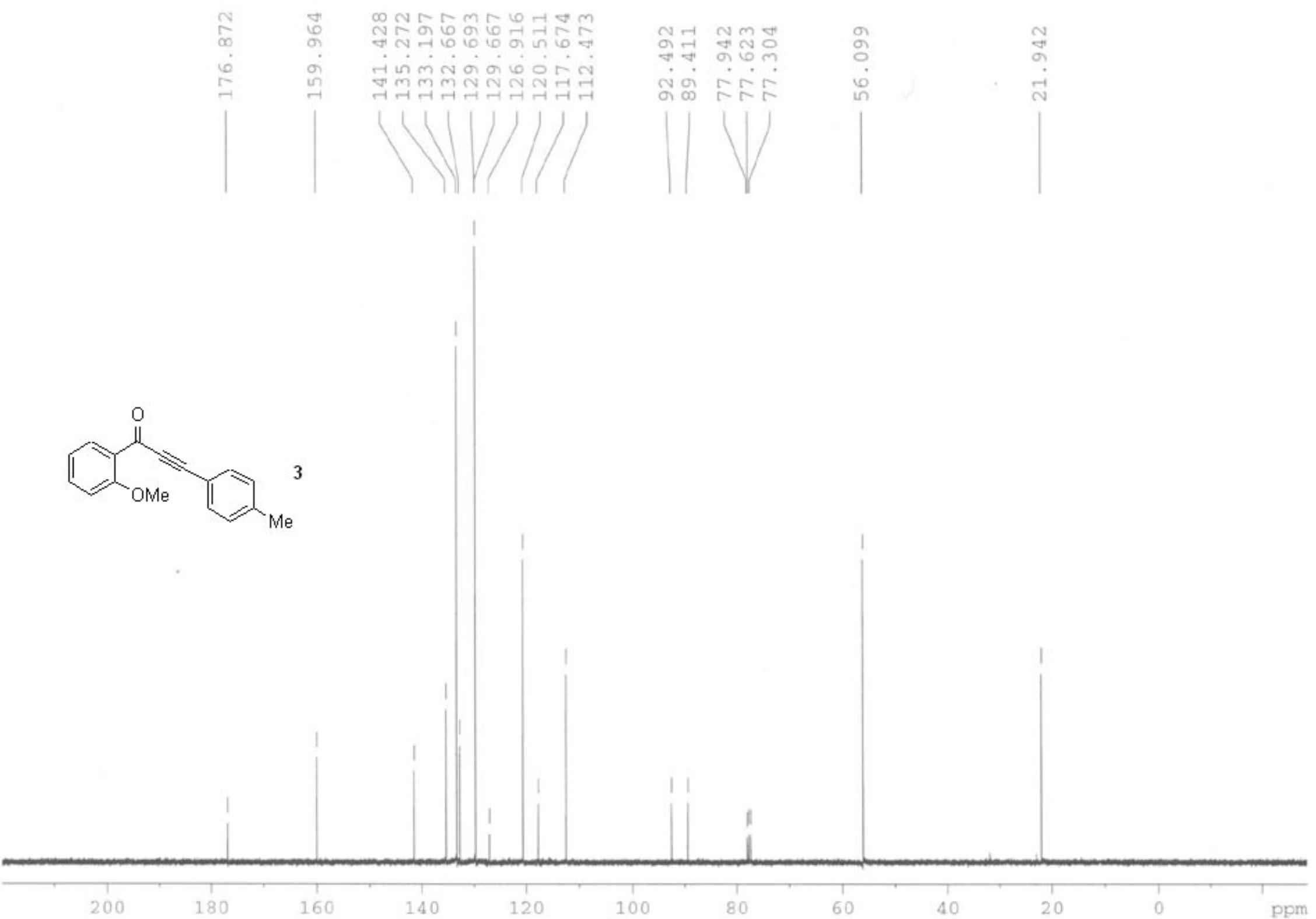


แn

nก

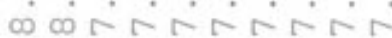
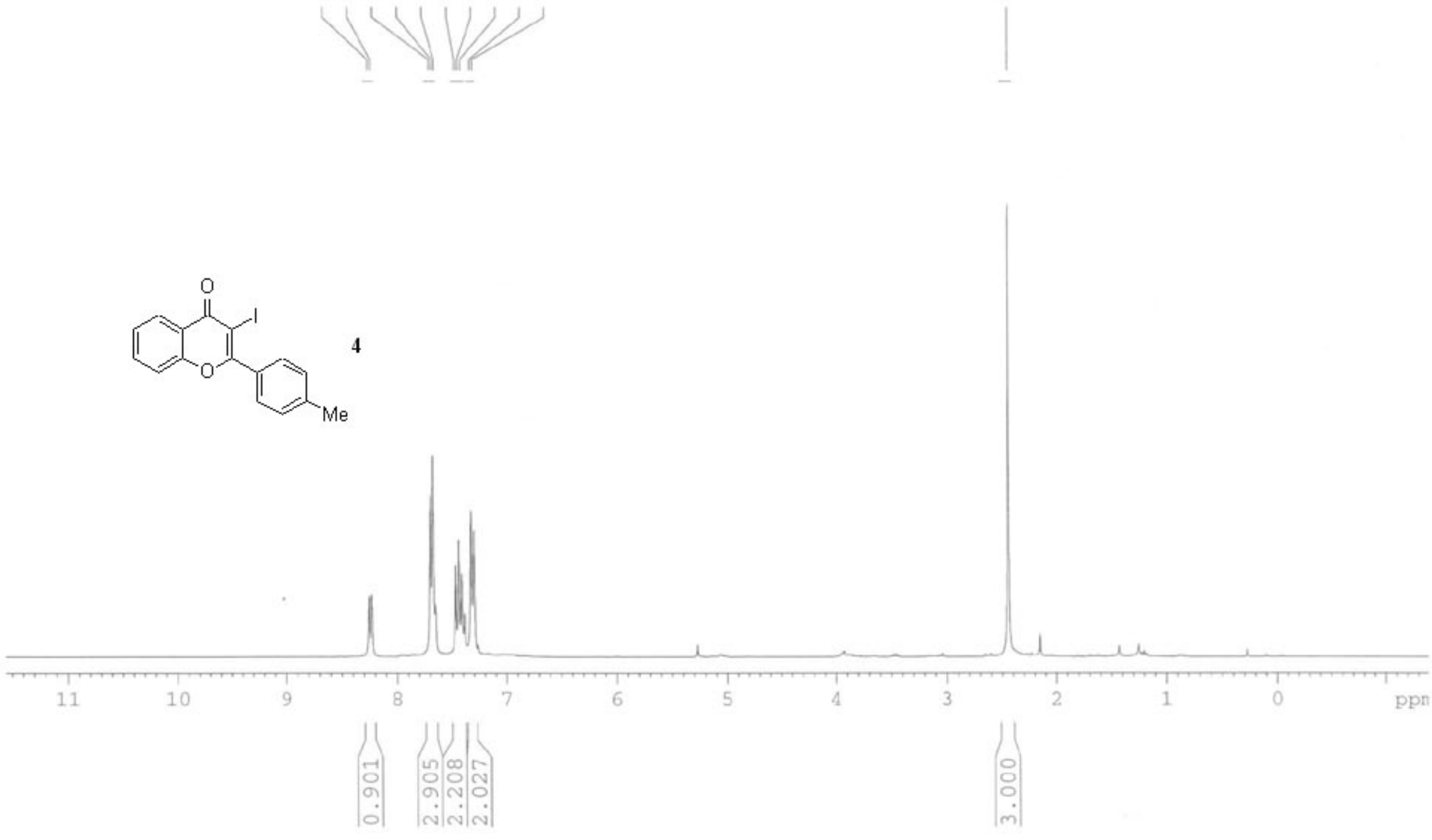

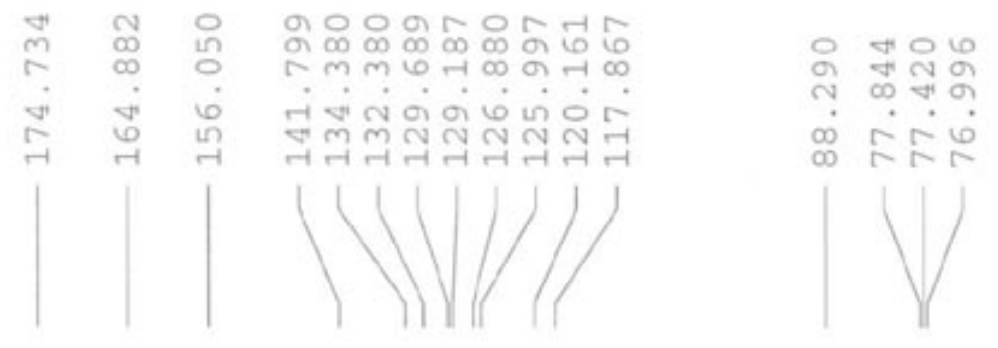

$$
\begin{aligned}
& \text { 며 } \\
& \text { बे } \\
& \text { ते }
\end{aligned}
$$<smiles>O=c1c(I)c(-c2ccc([N+](=O)[O-])cc2)oc2ccccc12</smiles>

$\mathrm{Me}$

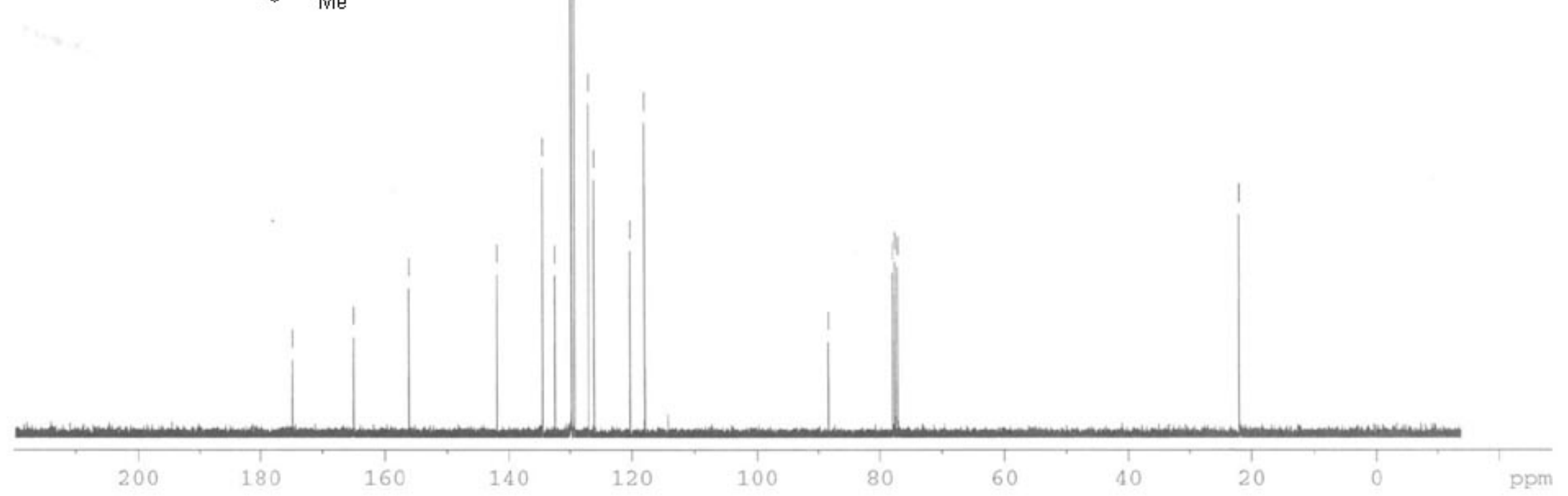




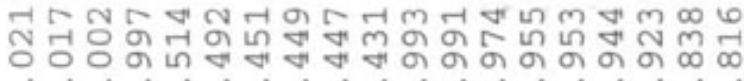

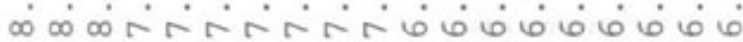

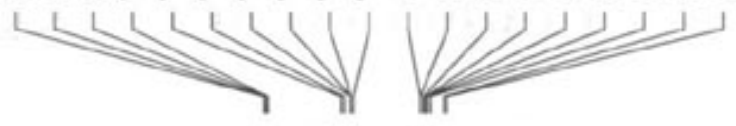

$\stackrel{n}{\pi} \stackrel{0}{r}$

$\infty \mathrm{r}$

$\dot{m} \dot{m}$

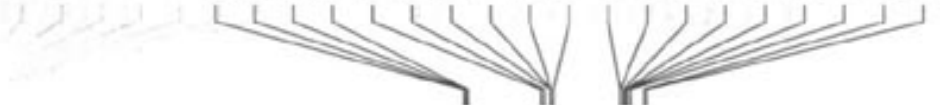

1
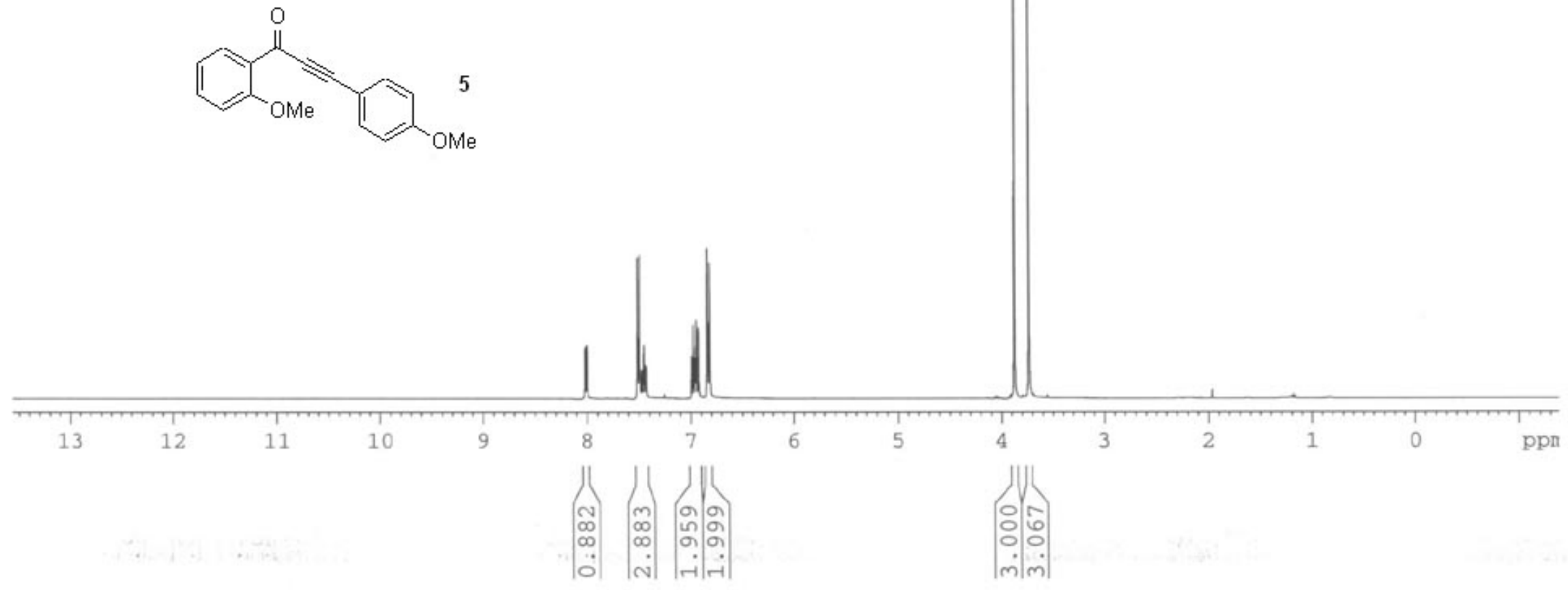


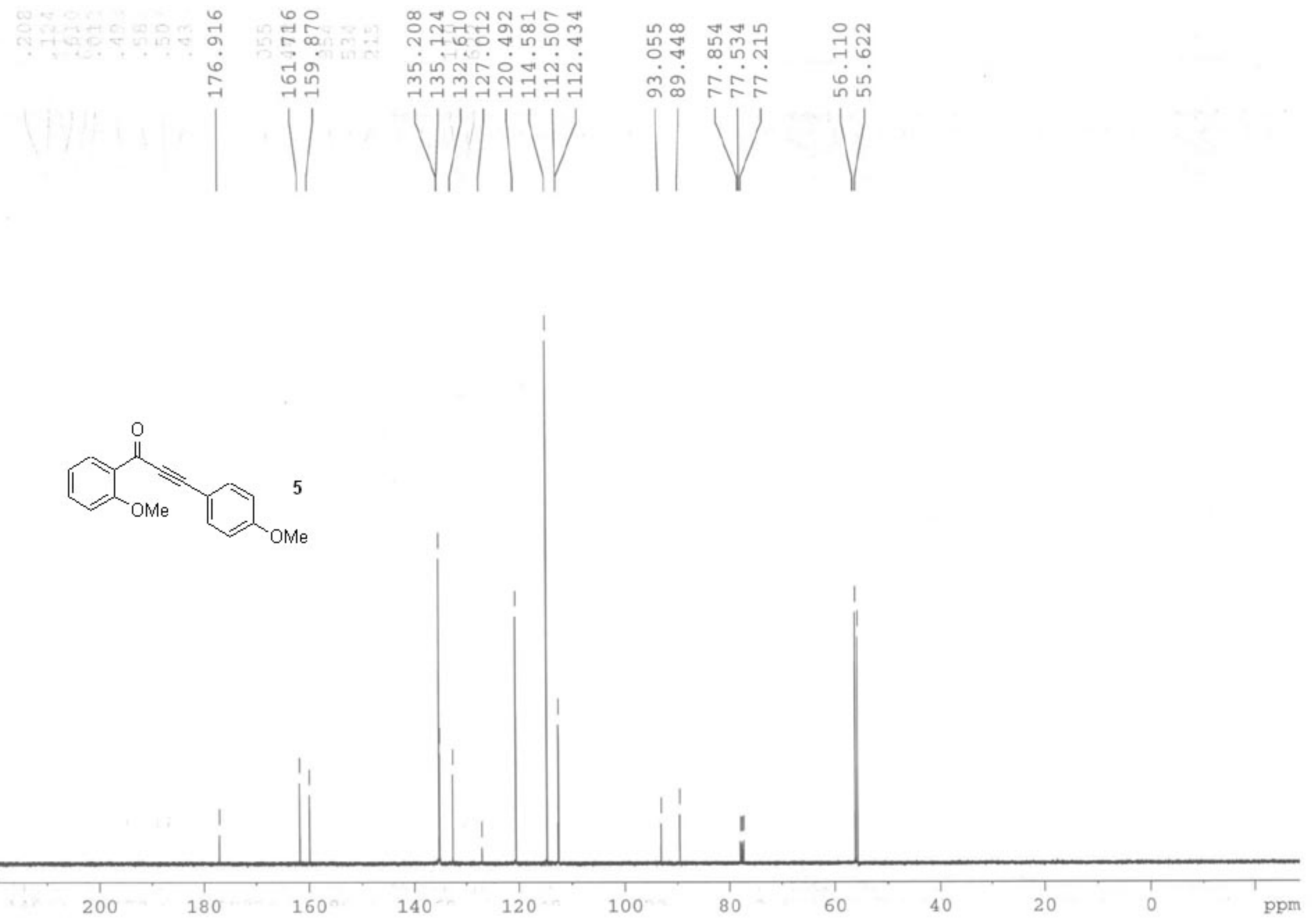



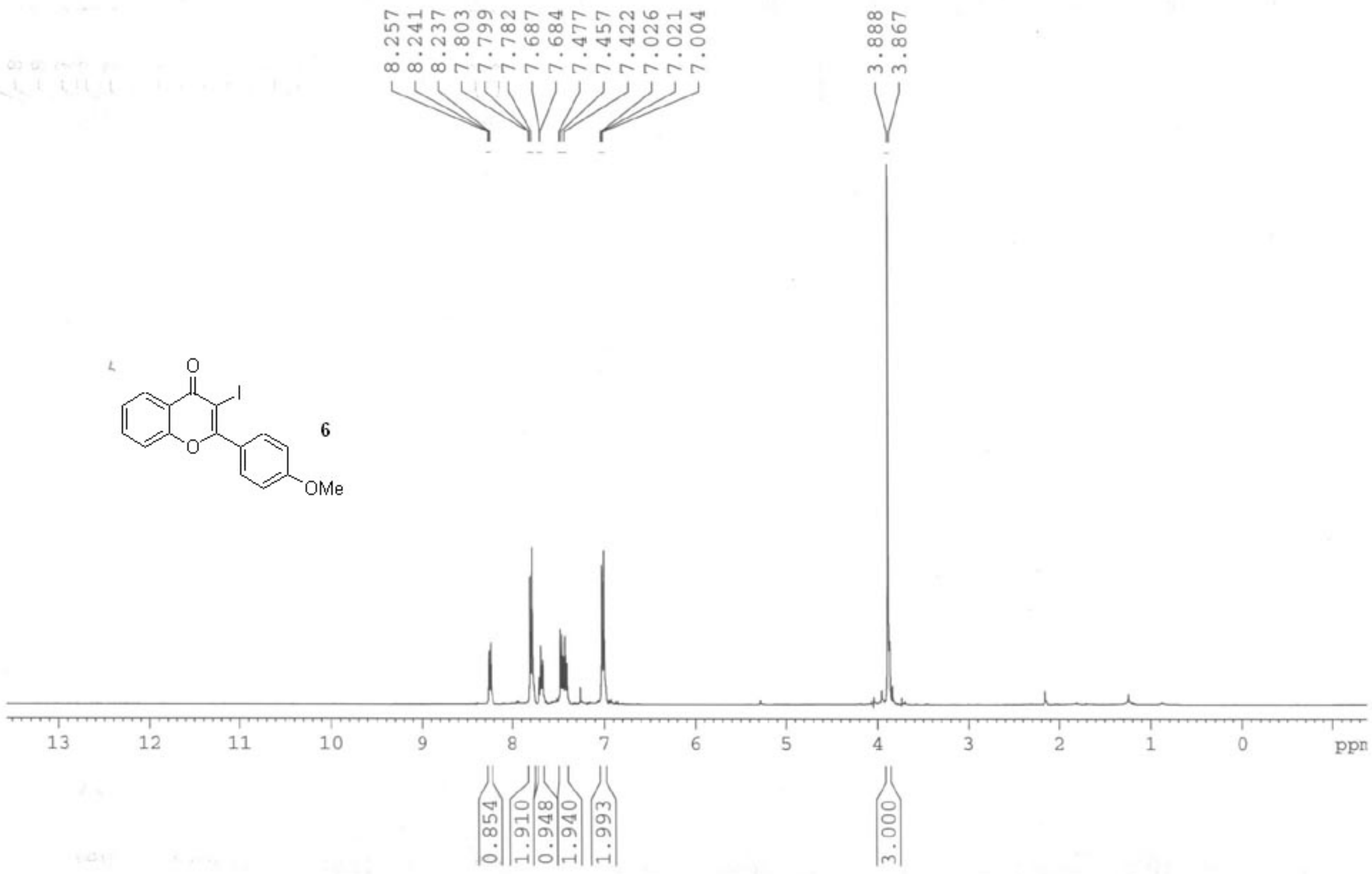

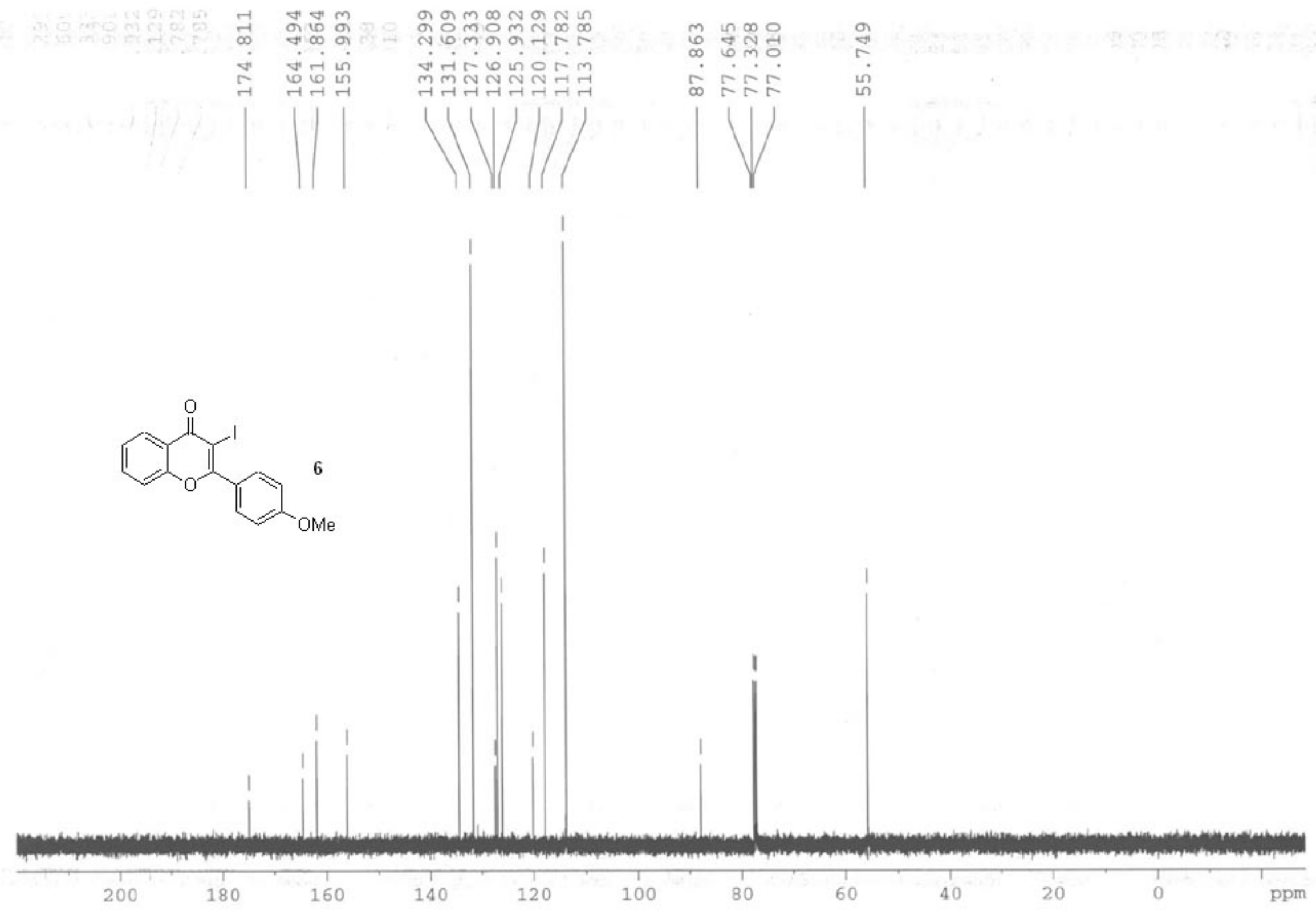

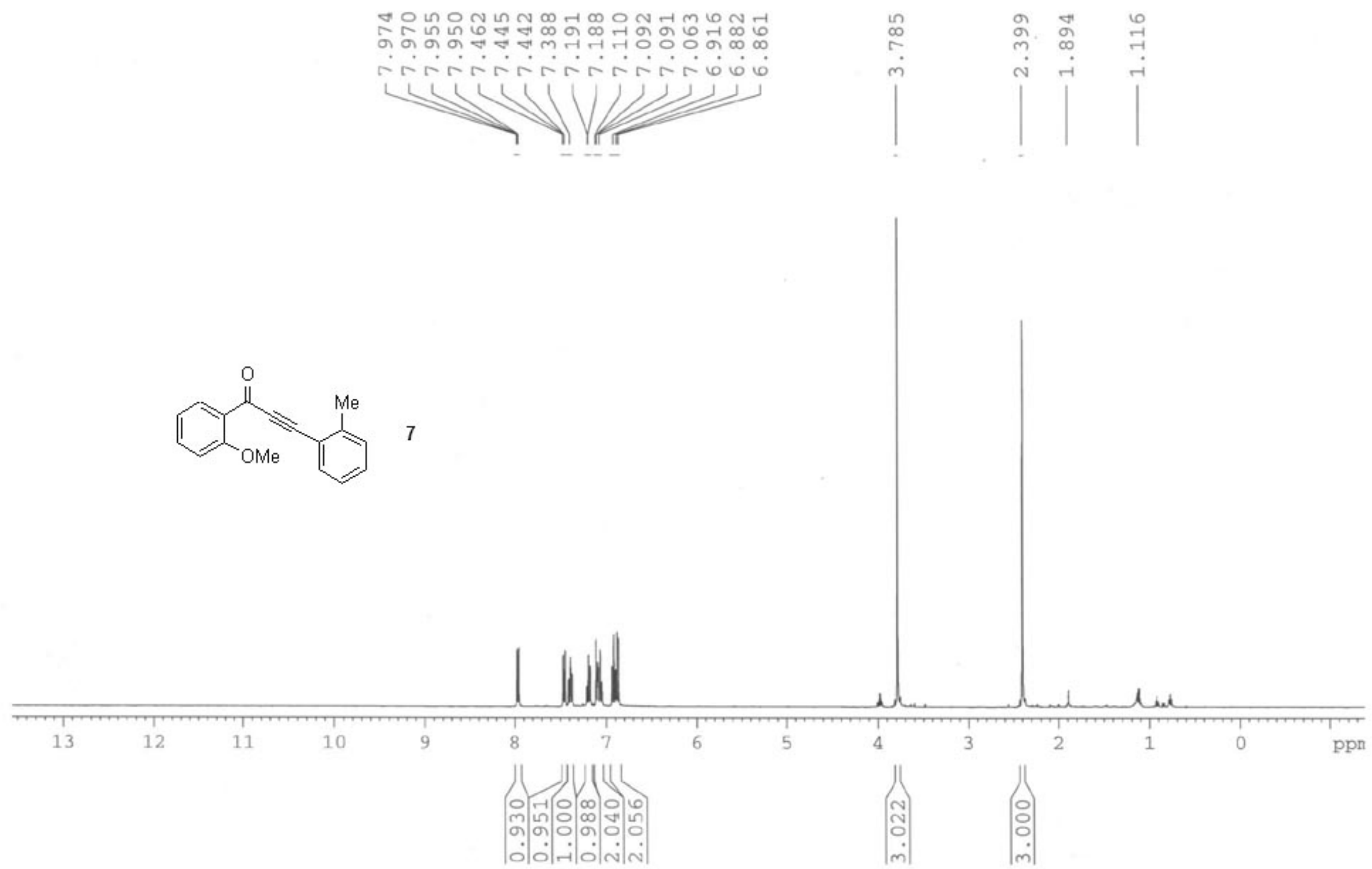

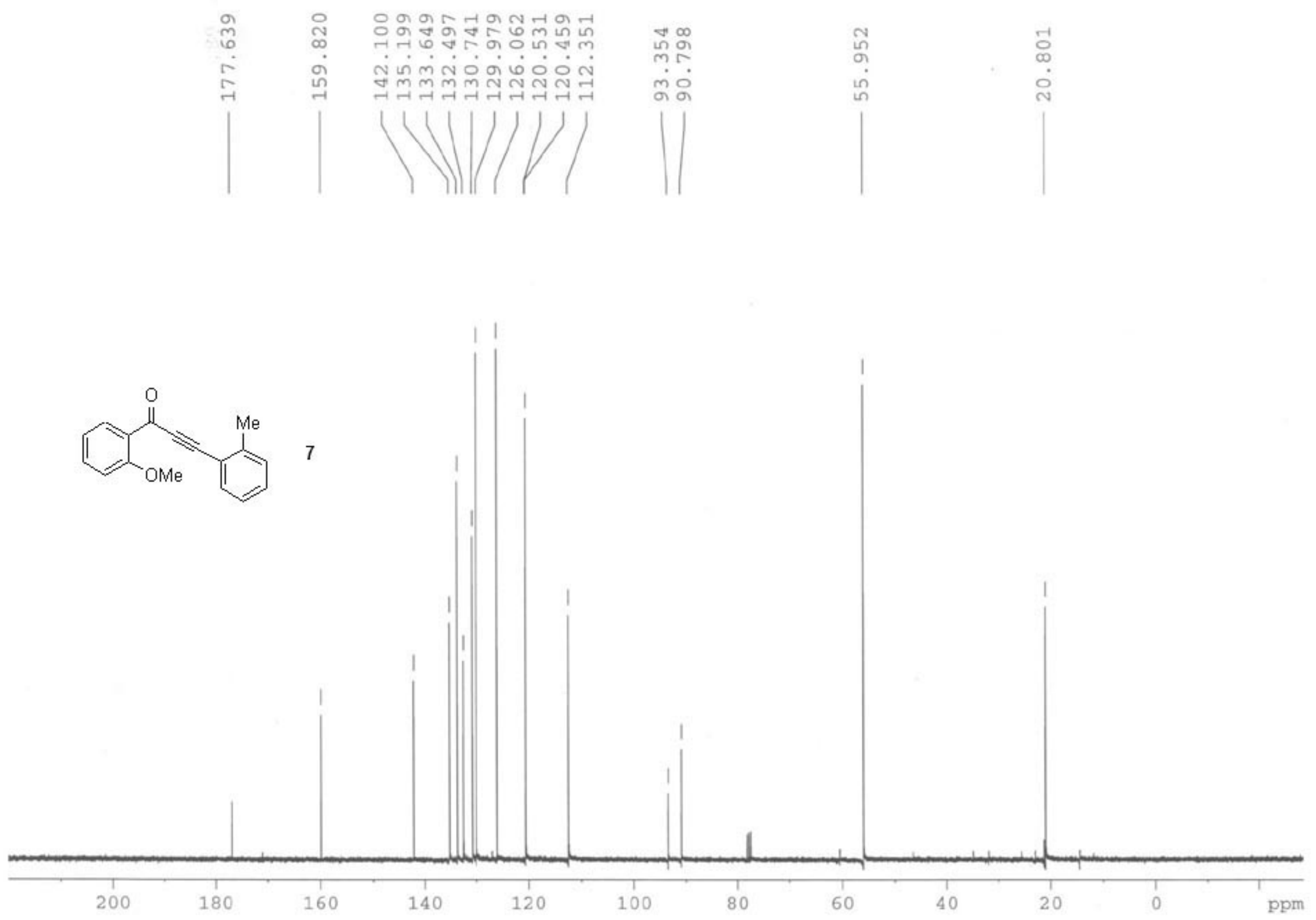


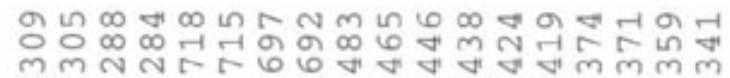

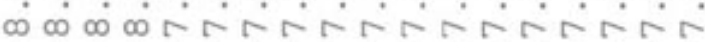
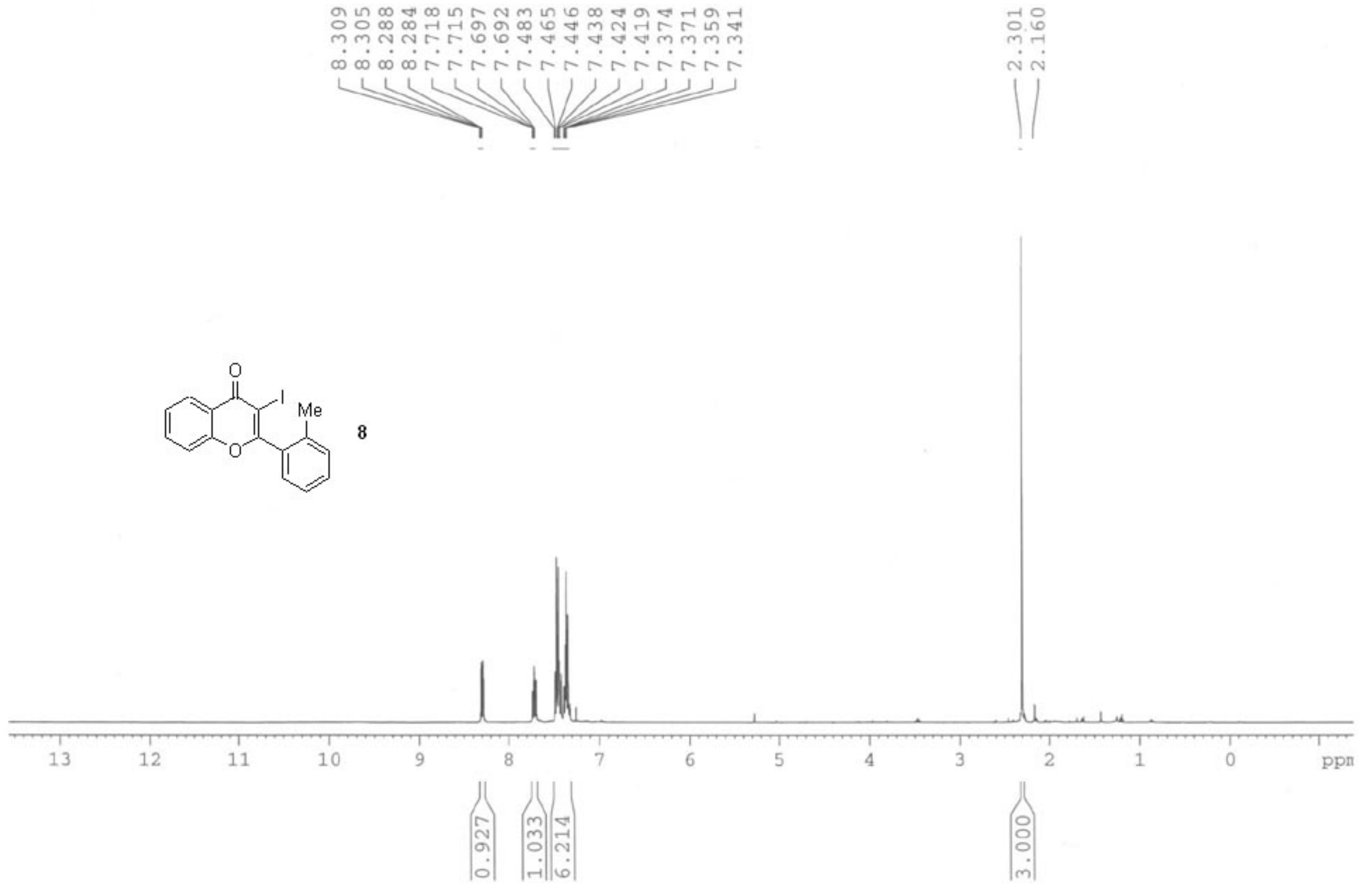

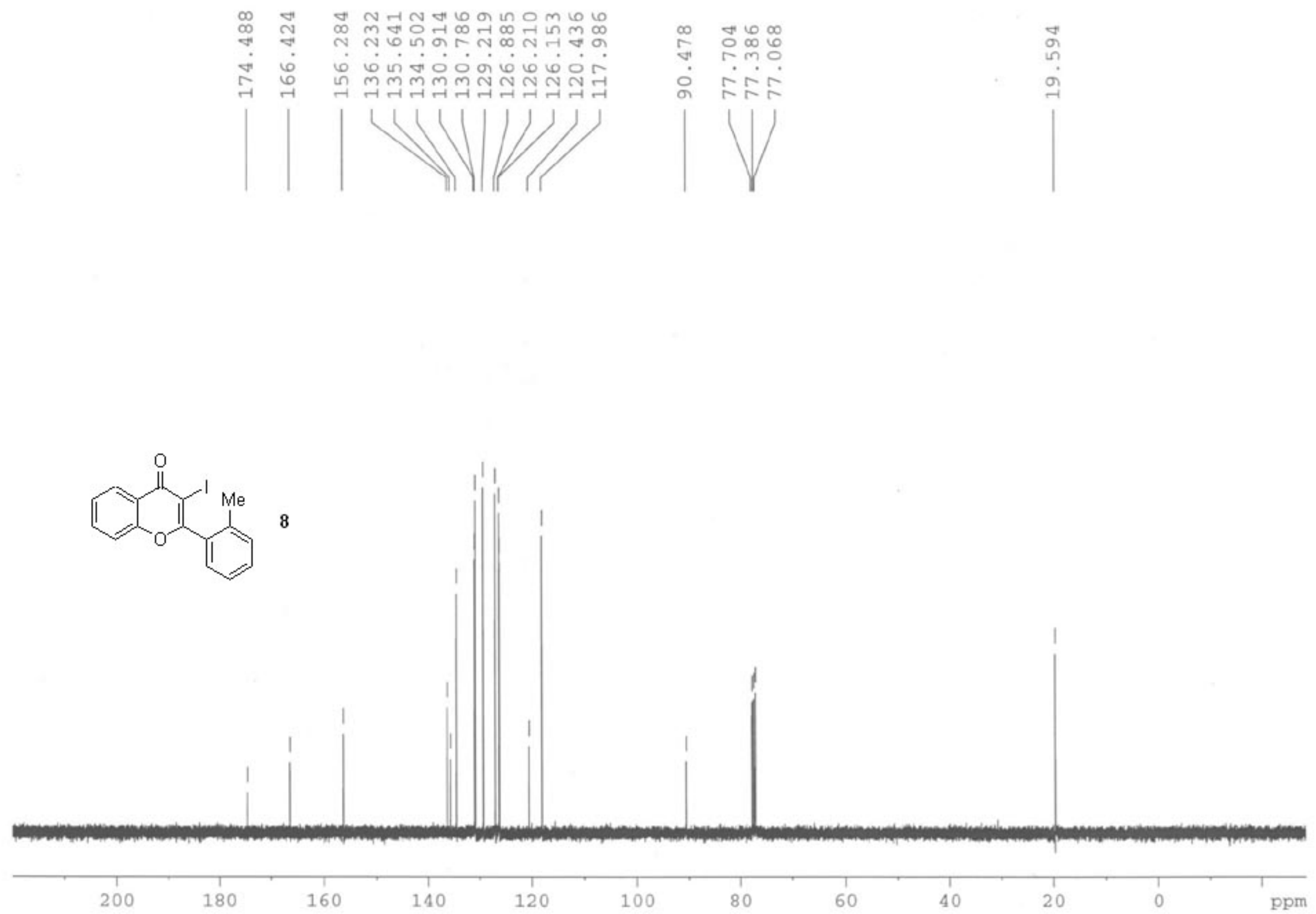
ำก

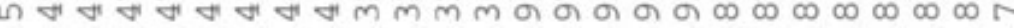

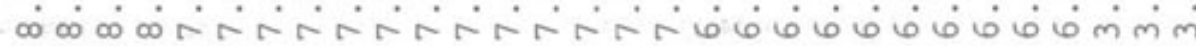

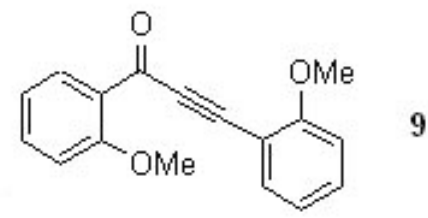

9

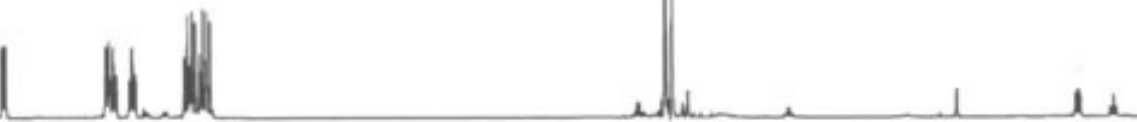

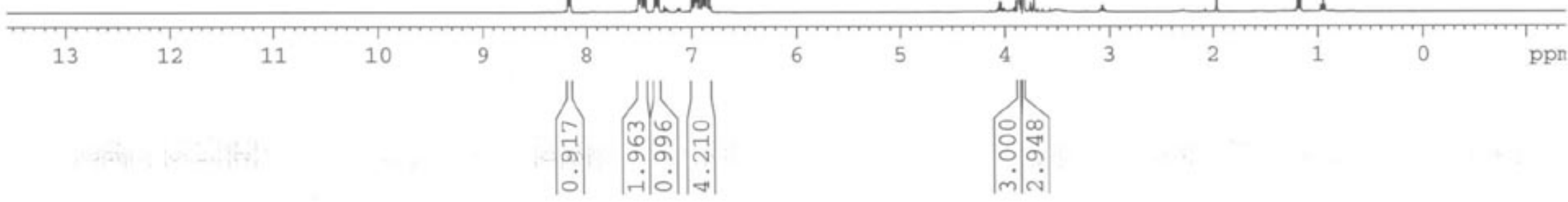




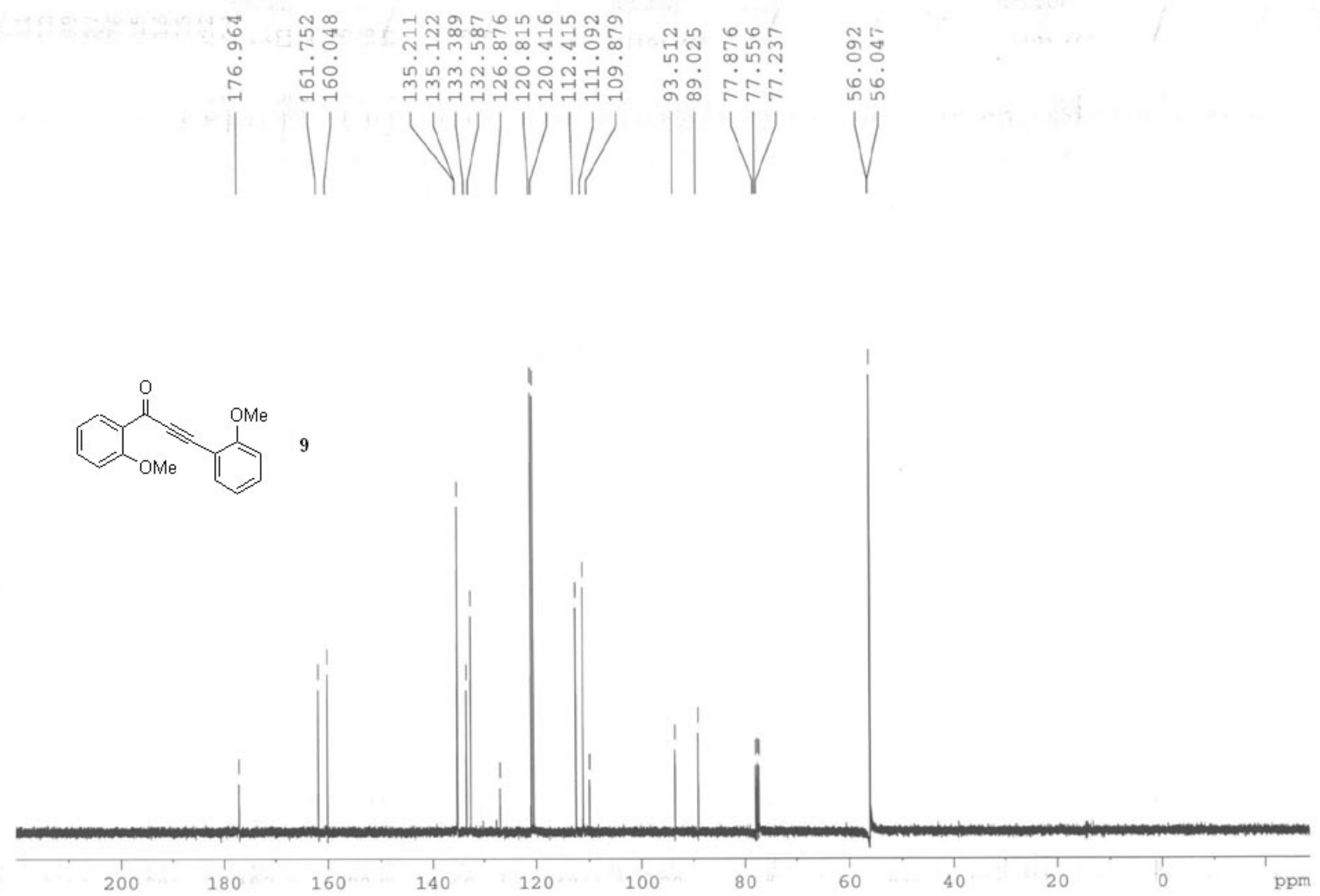


mov

곤

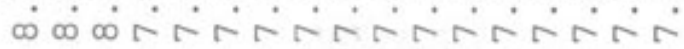

$1<<<<1 / 1 / 1 / 1)$

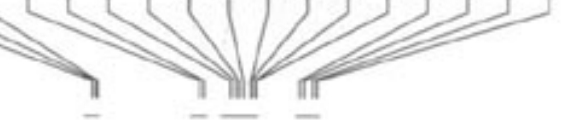

$\infty$

m
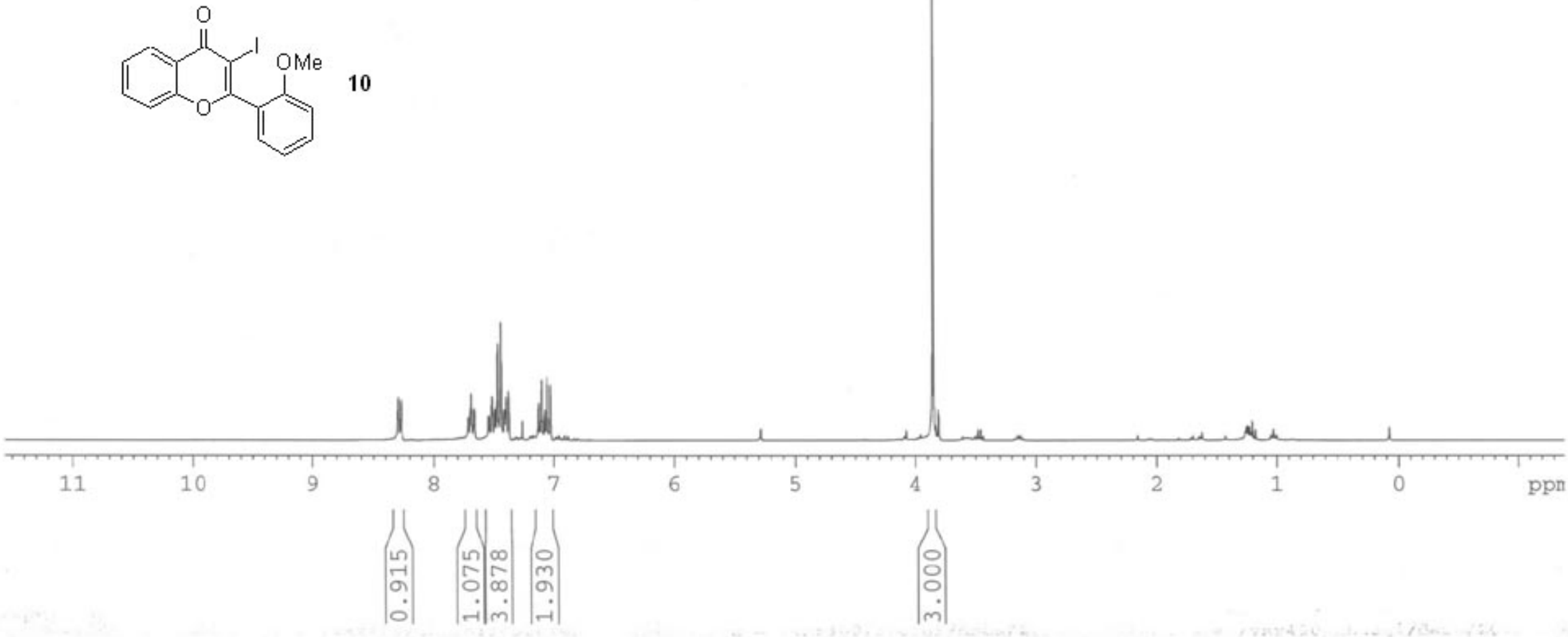

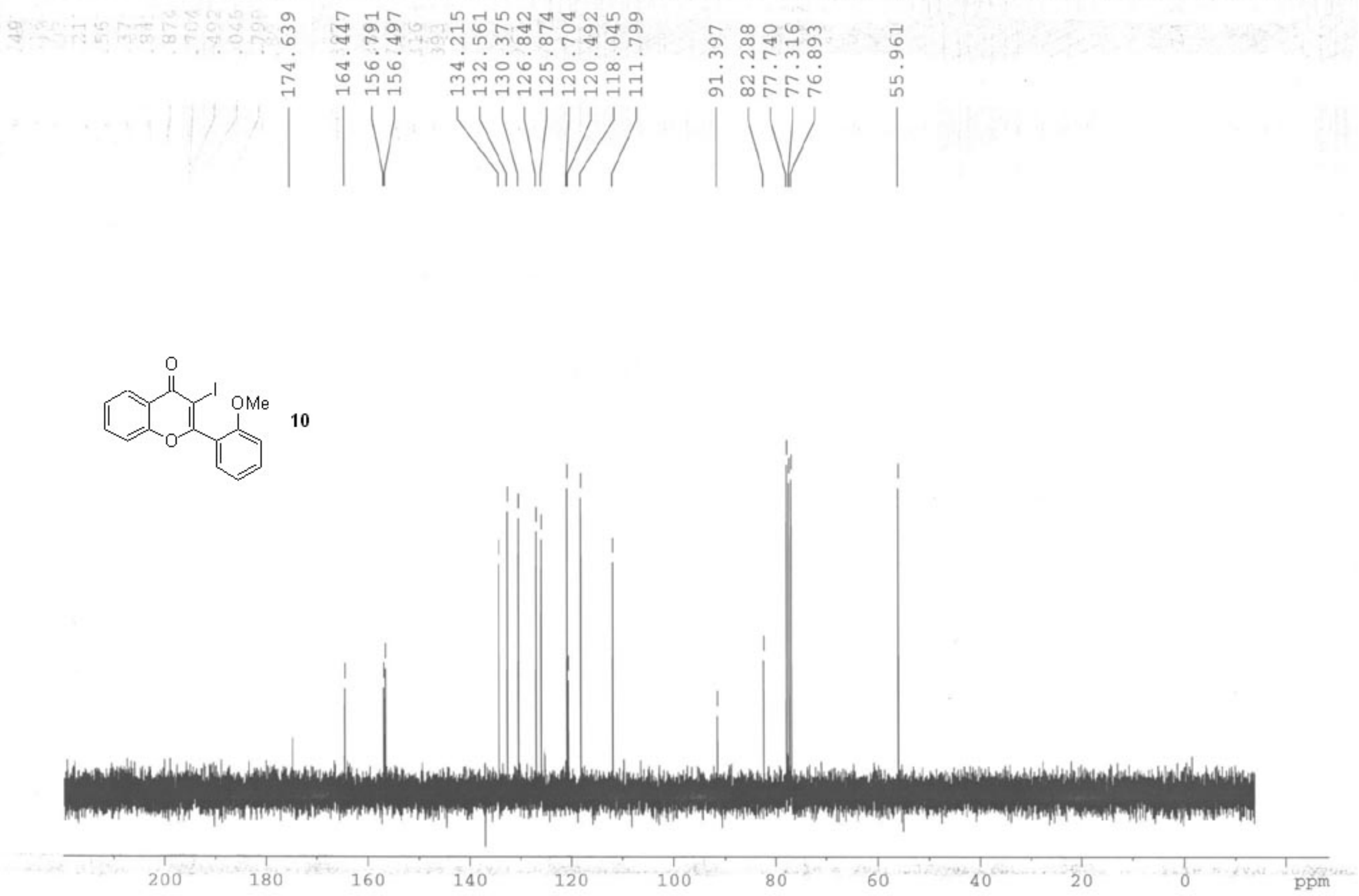

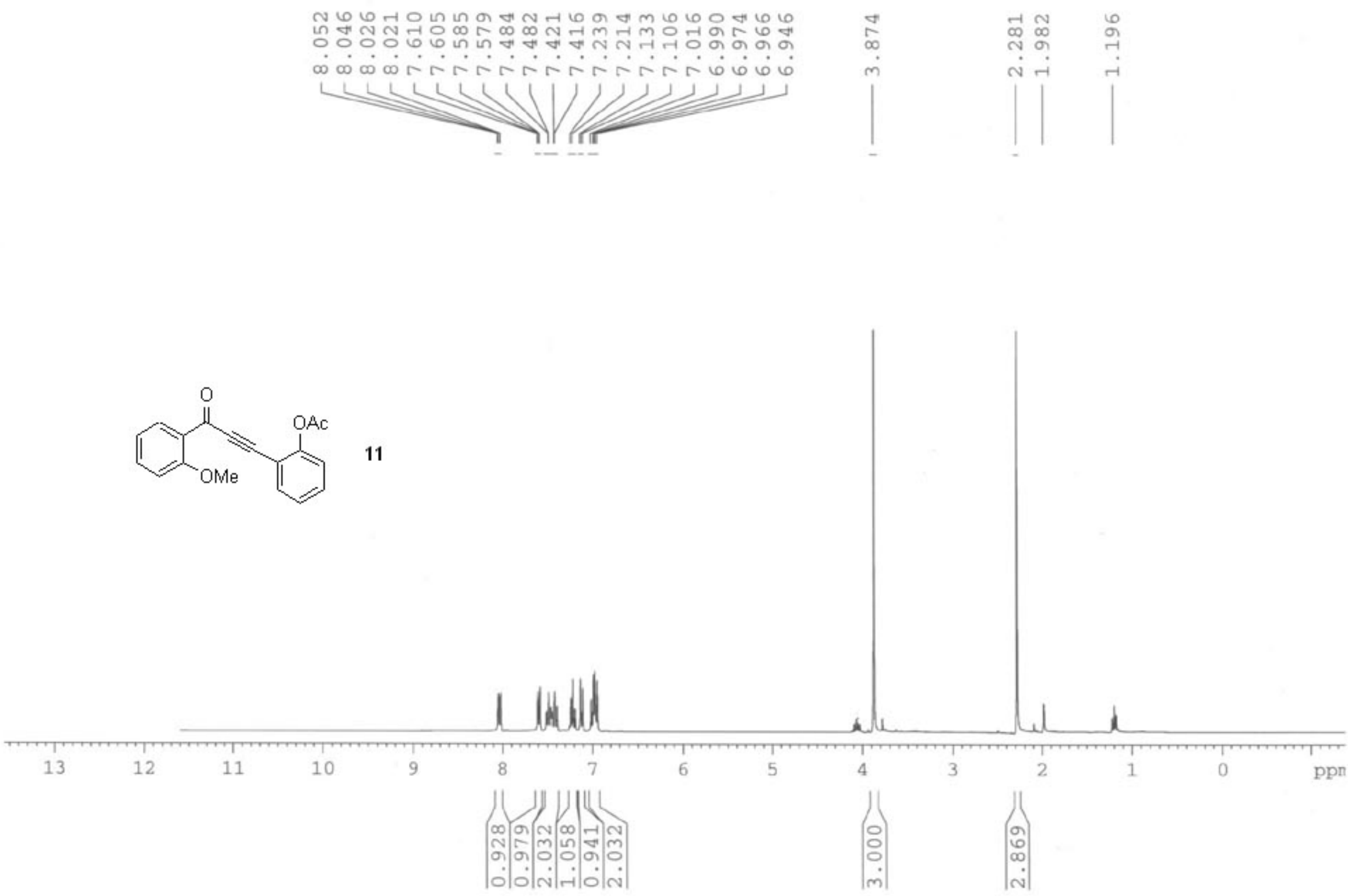


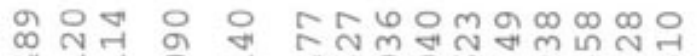

चन न

๒

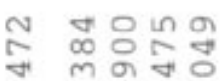

ले
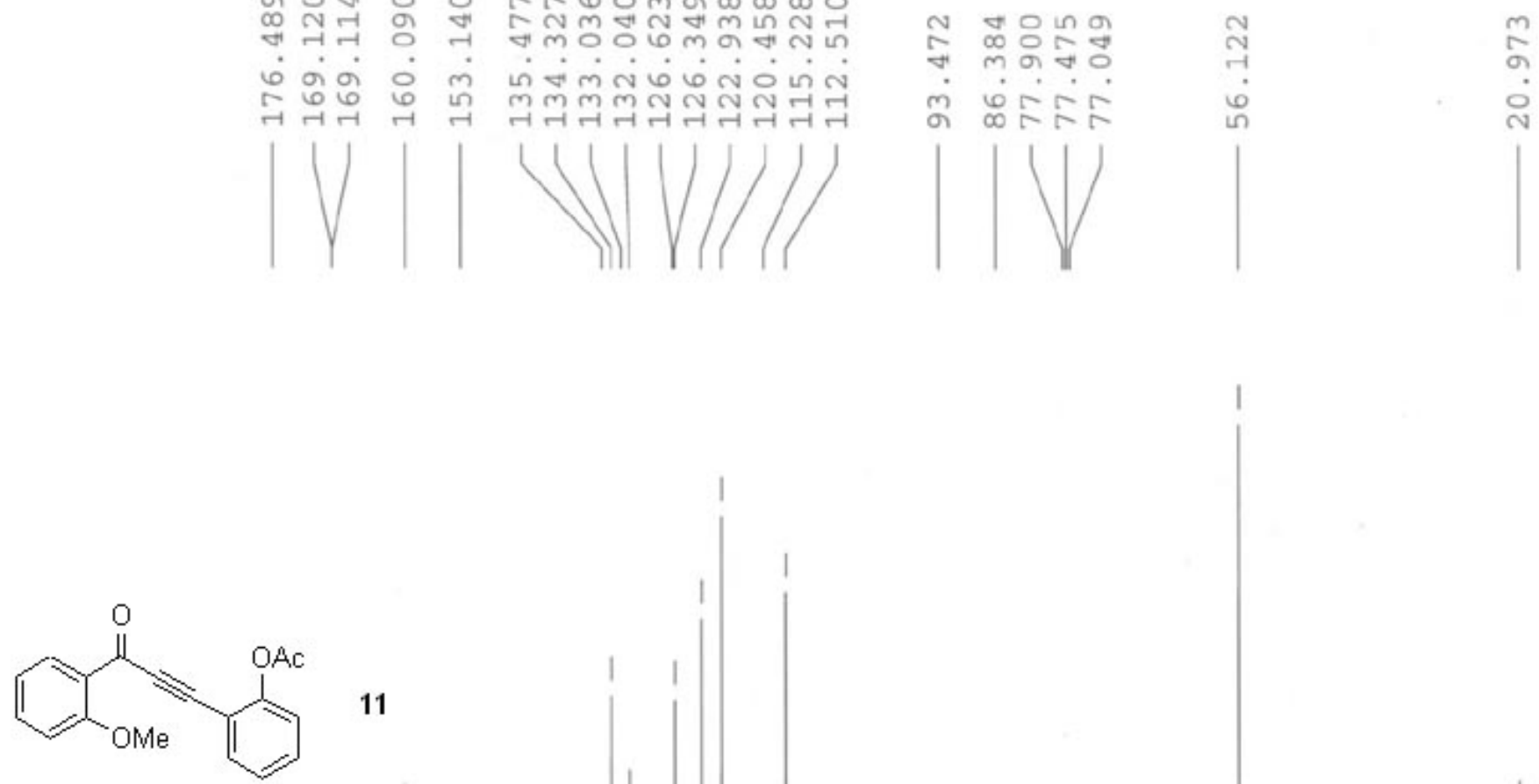

11

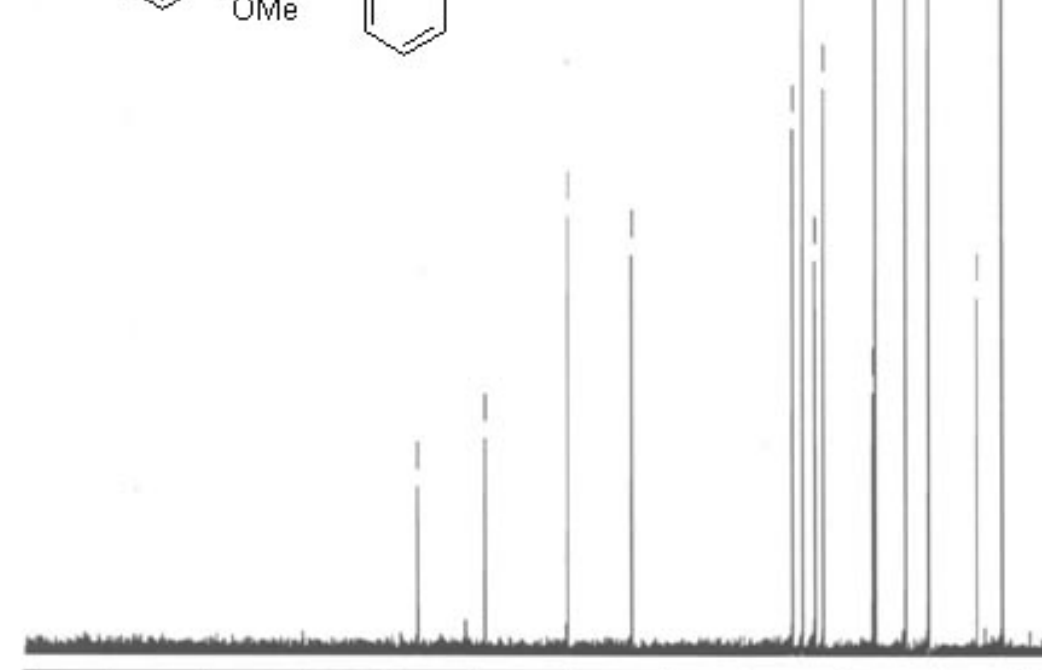

200

180

160

140

120

100

80

60

20

0

ppm 


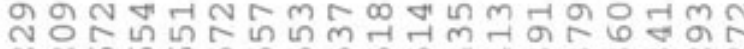

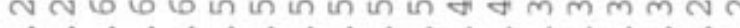

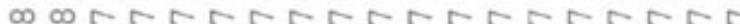

$\rightarrow 0$

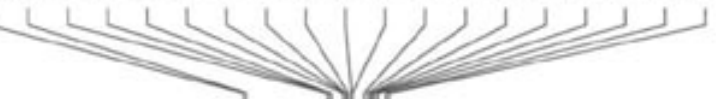

inim
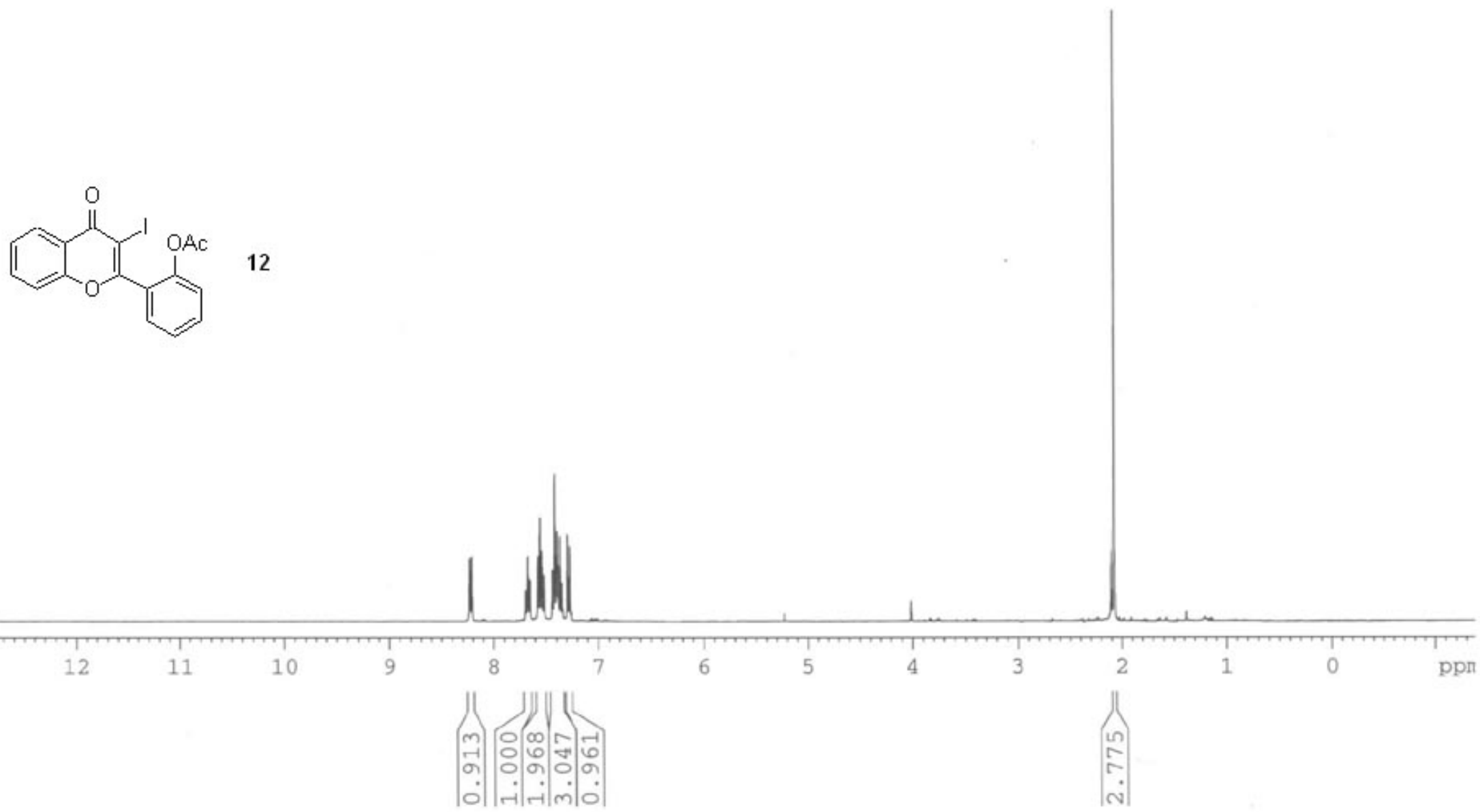


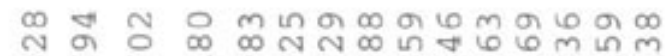

m $N$ ब

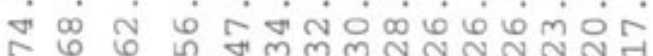

담

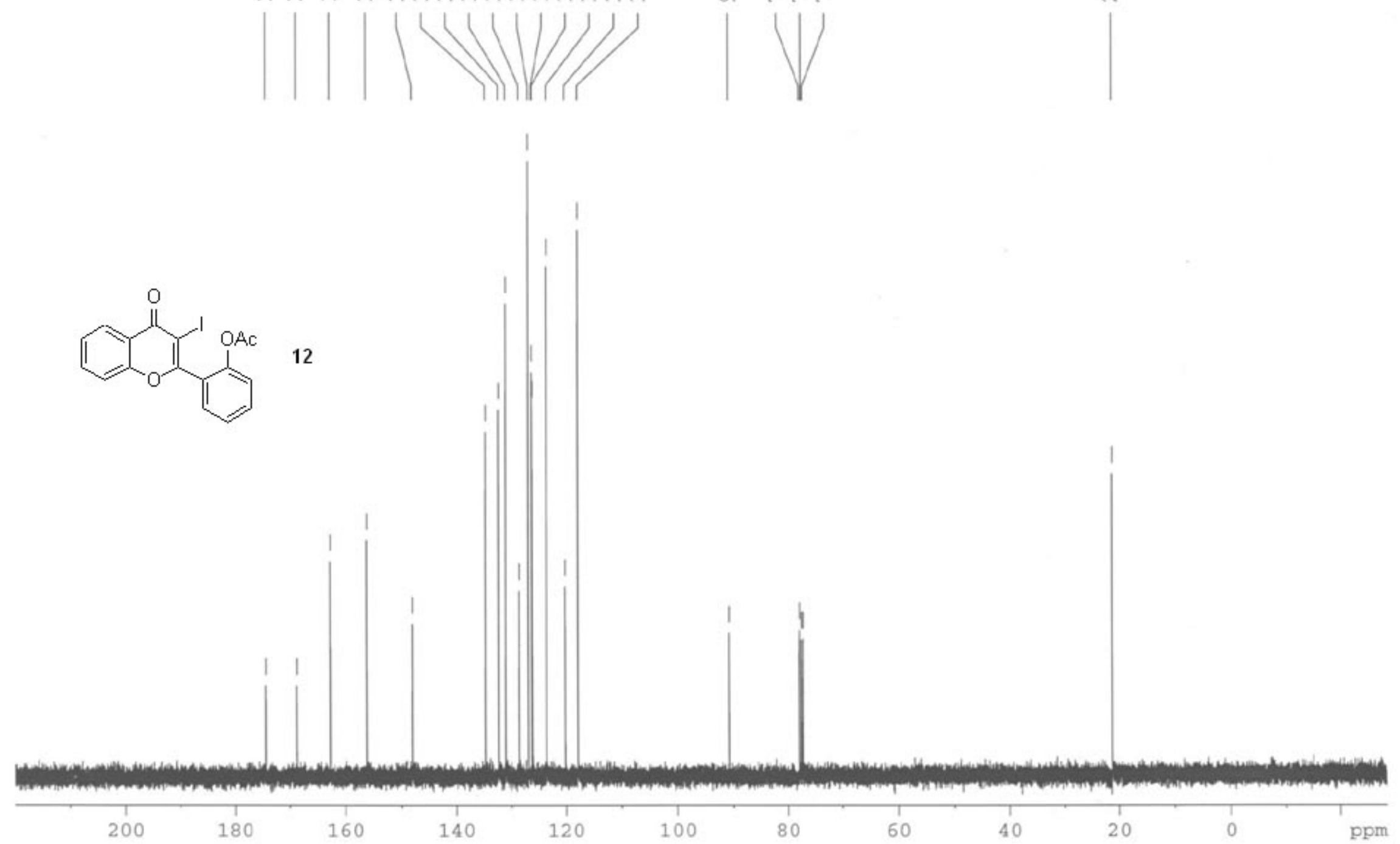

$\infty \quad \forall 6$

r. $\infty$ Ln

o rar

$\stackrel{\infty}{\infty}$

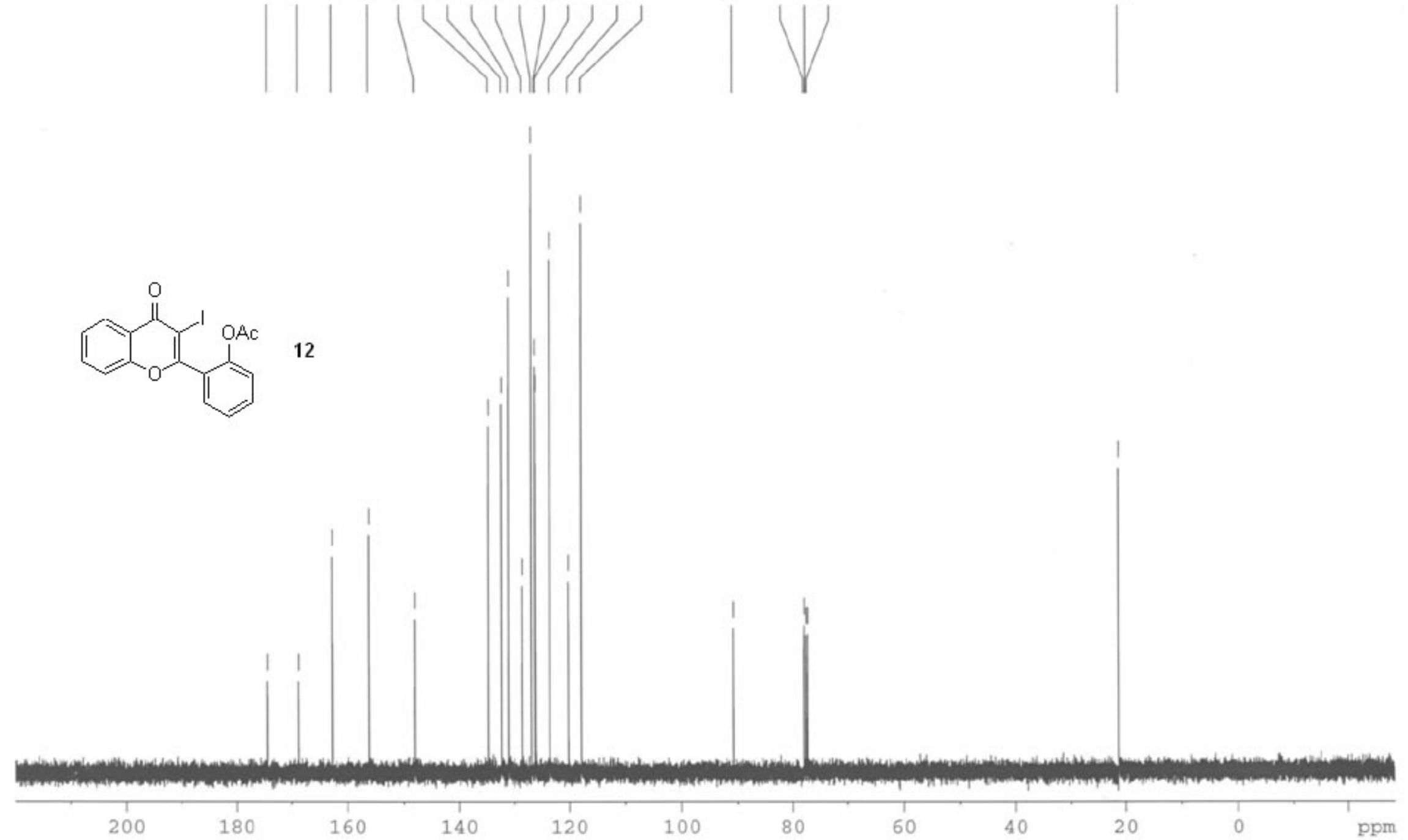


부대

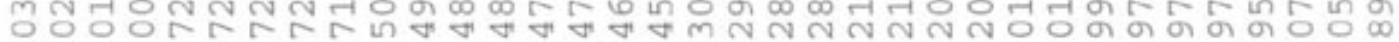

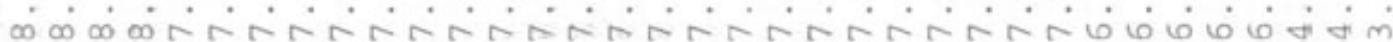

(l)

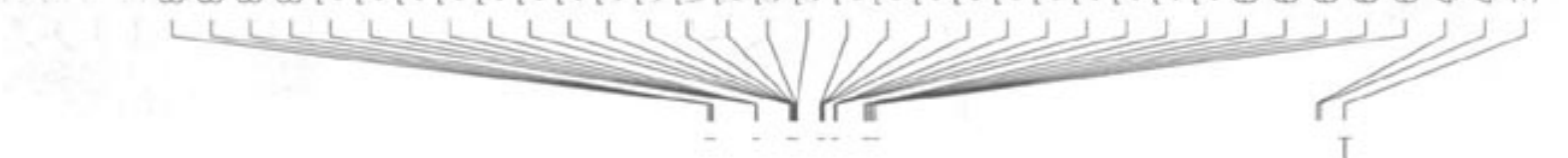

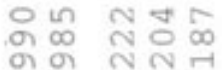

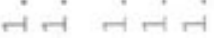
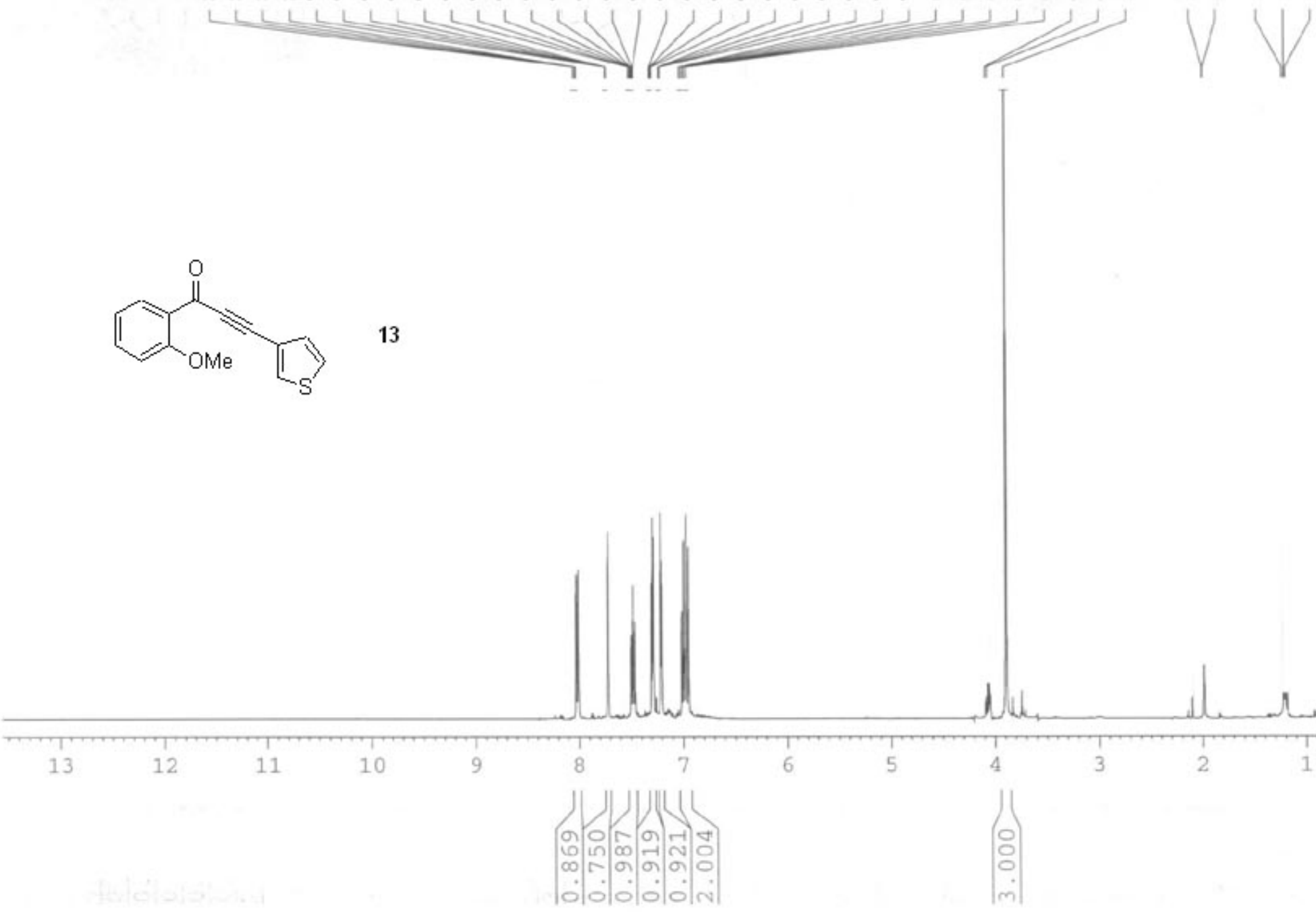

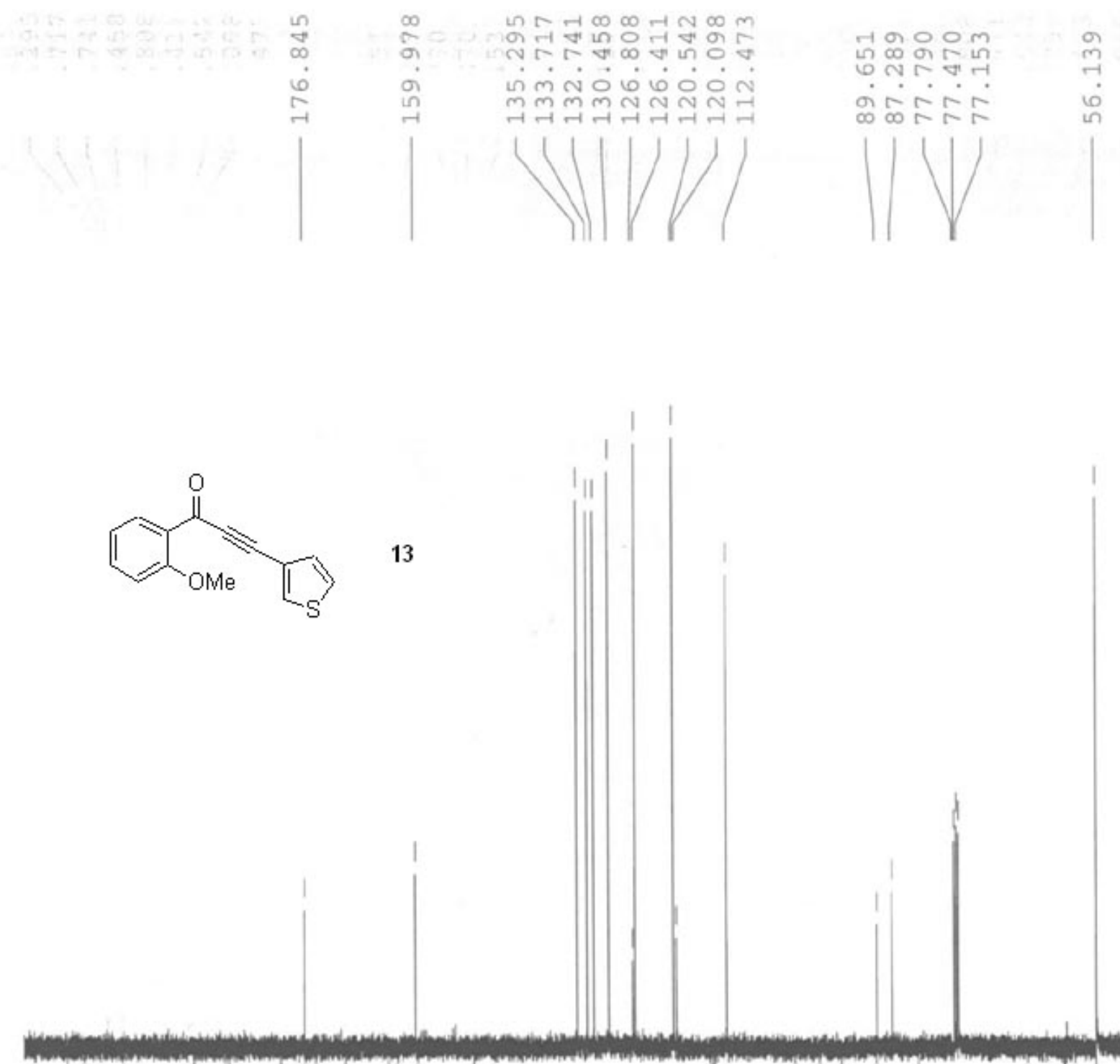
m

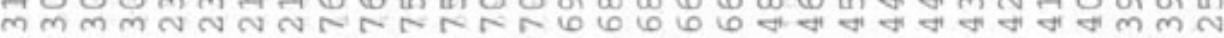

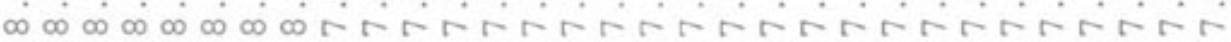

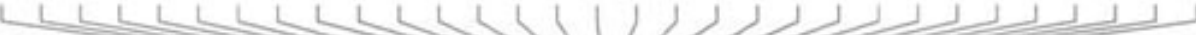

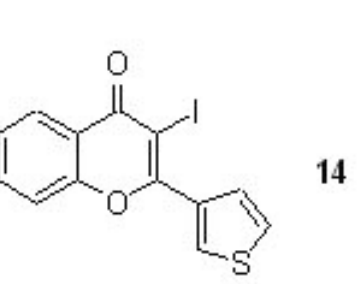

14

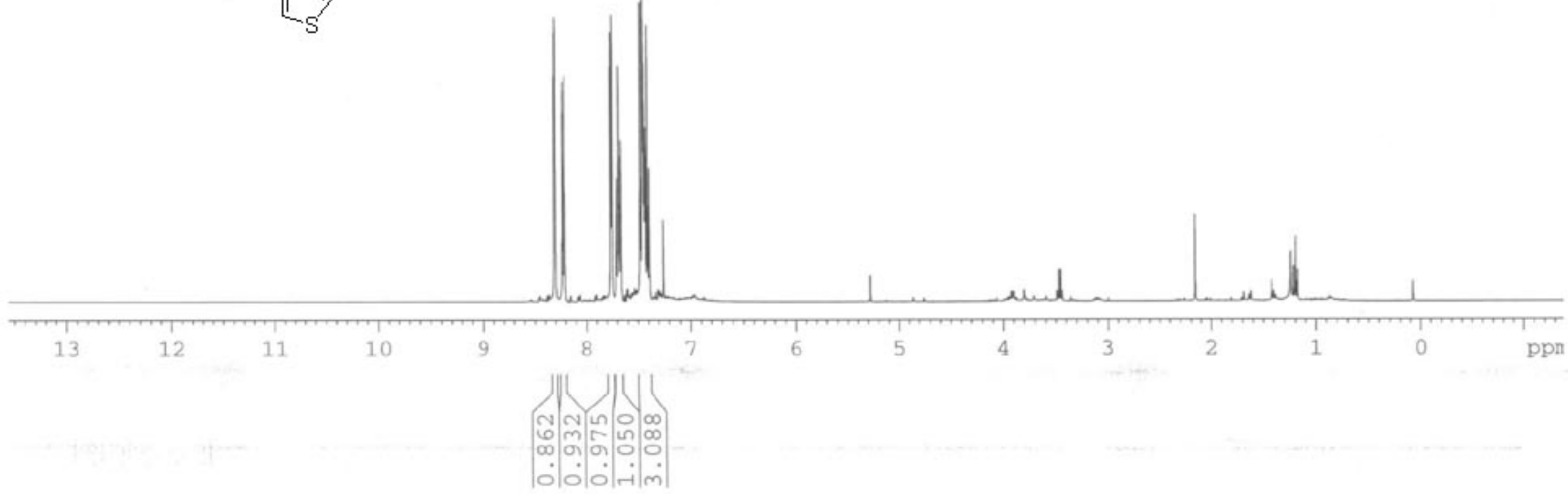




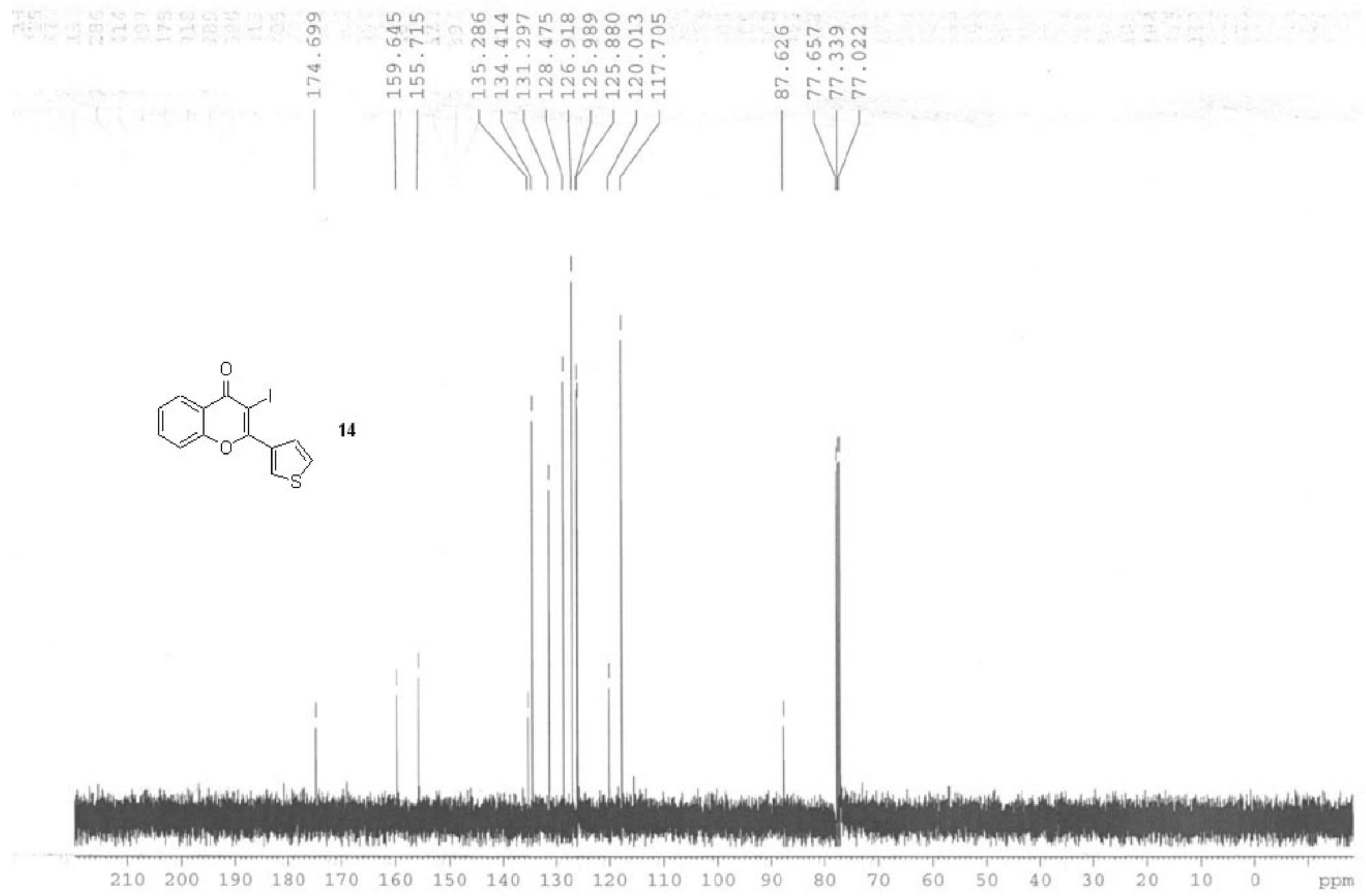

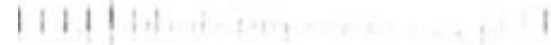




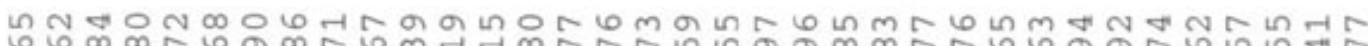

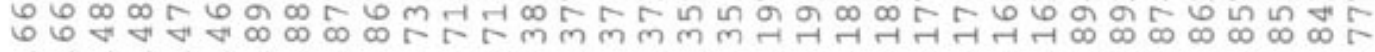

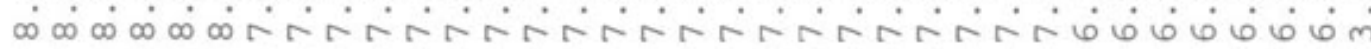

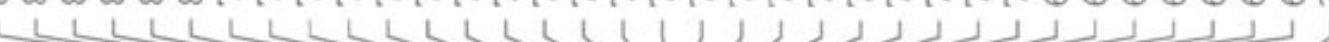
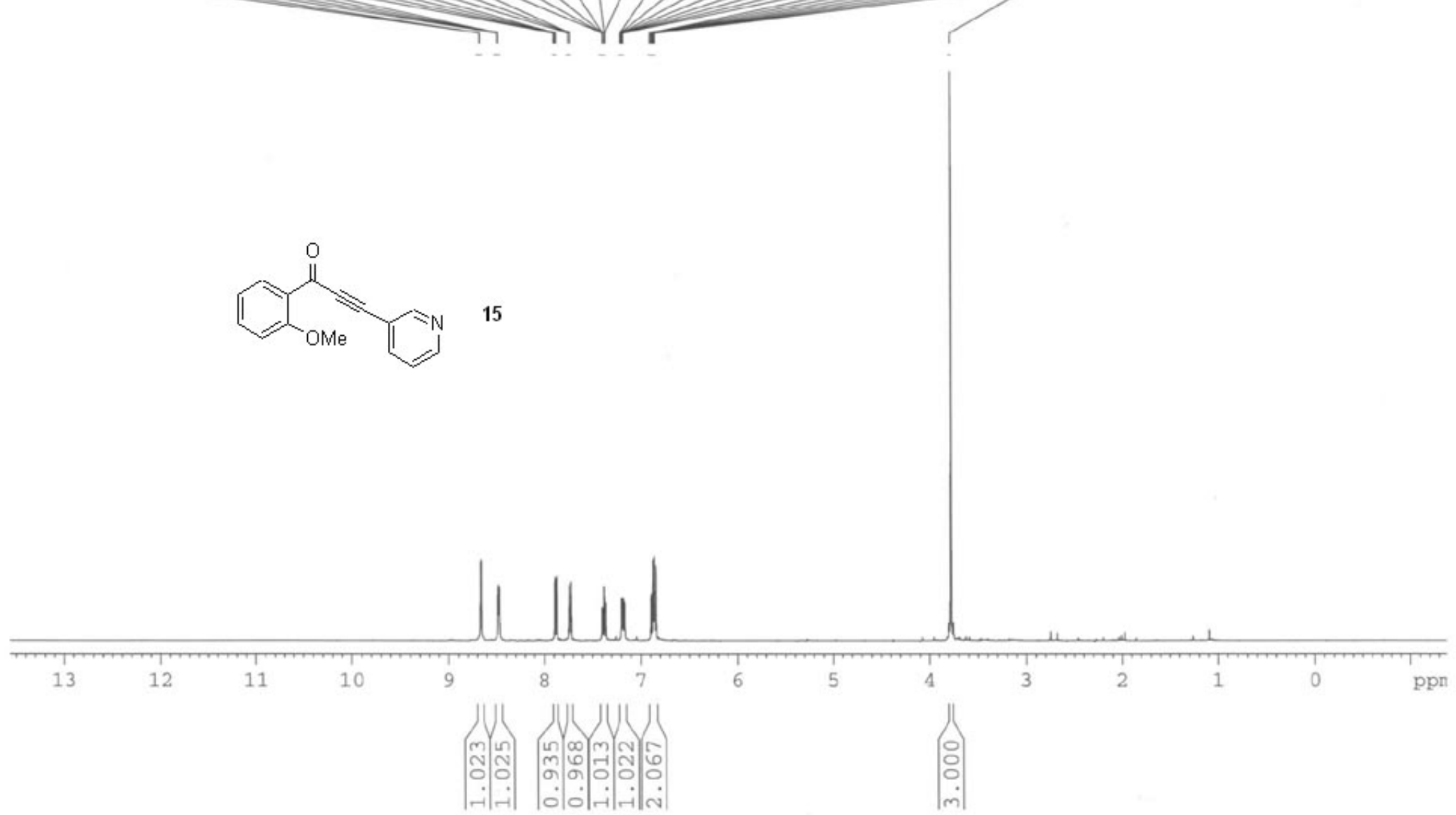

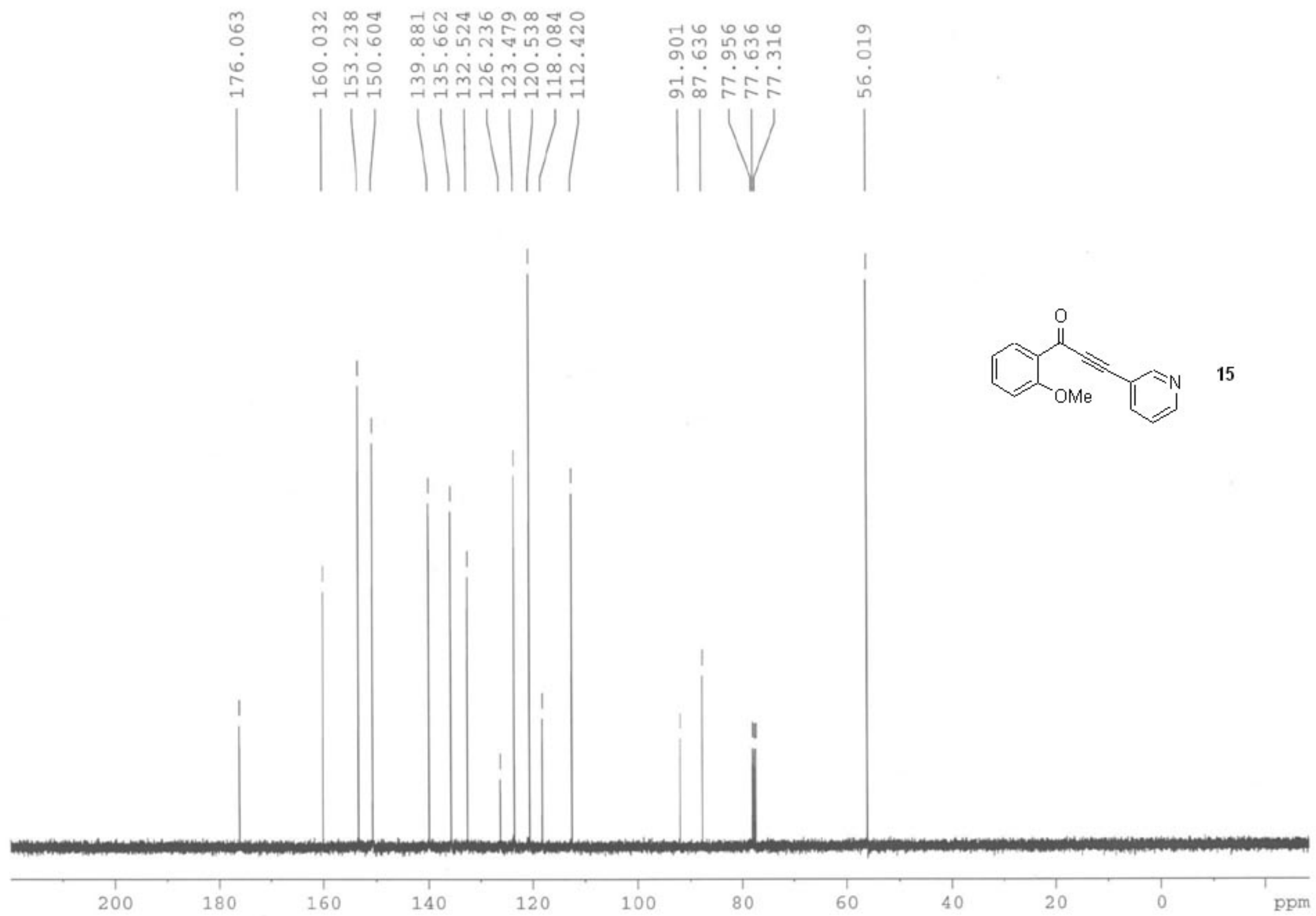


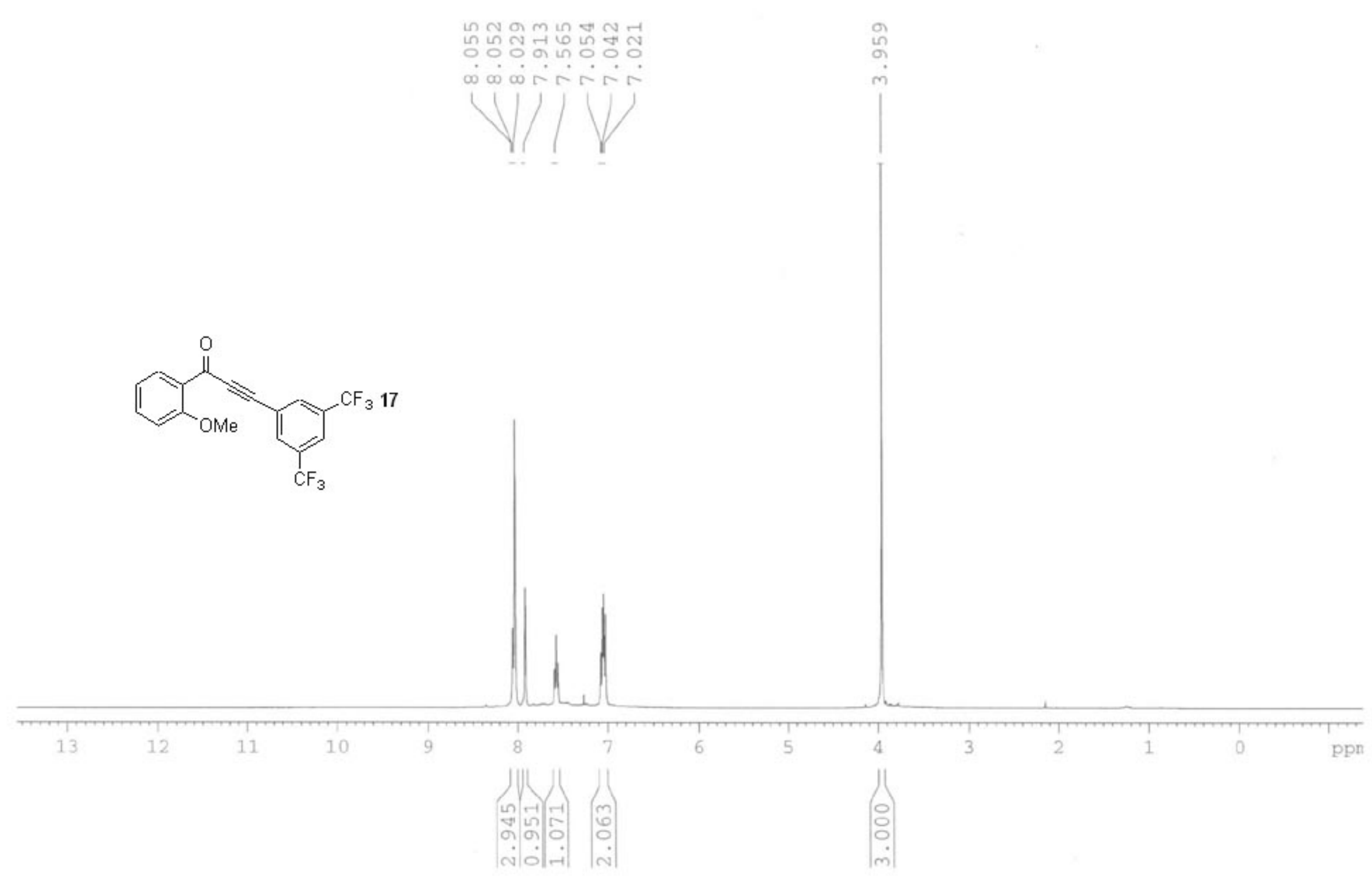




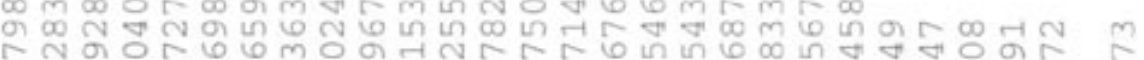

1िं

K
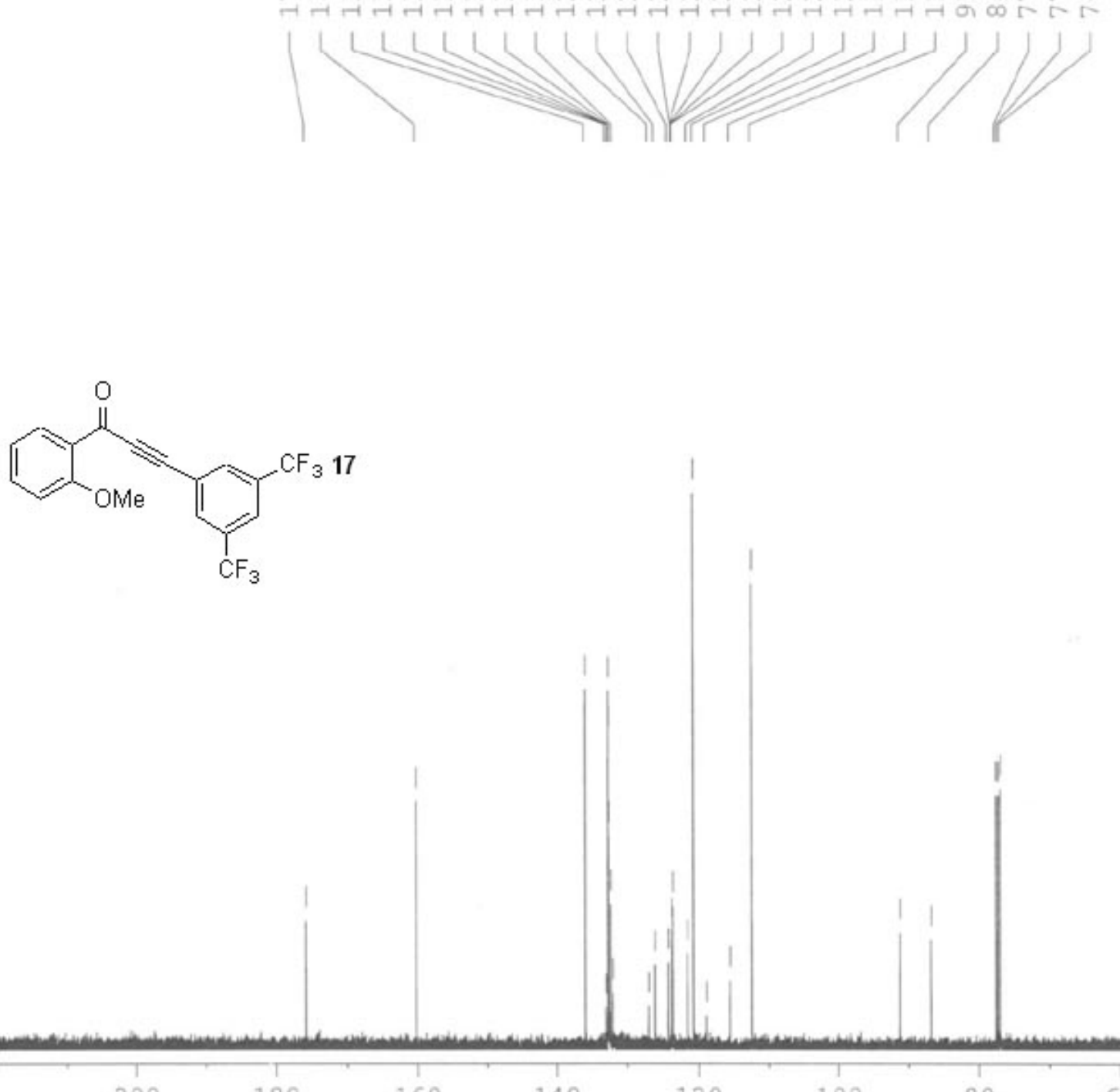

200

160

140

120

100

80

60

40

20 
$\infty \backsim \forall \infty$ n

制

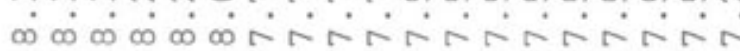
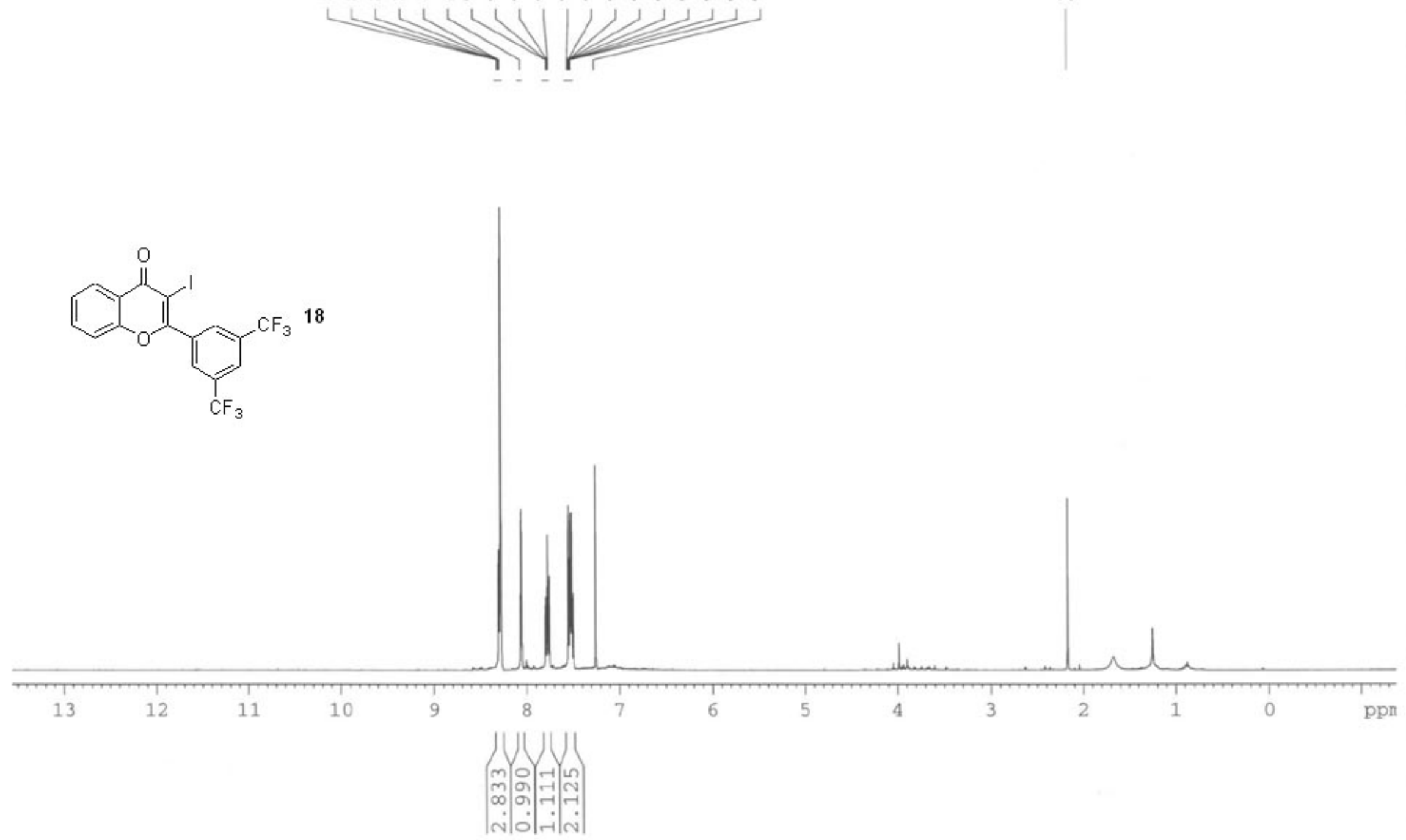


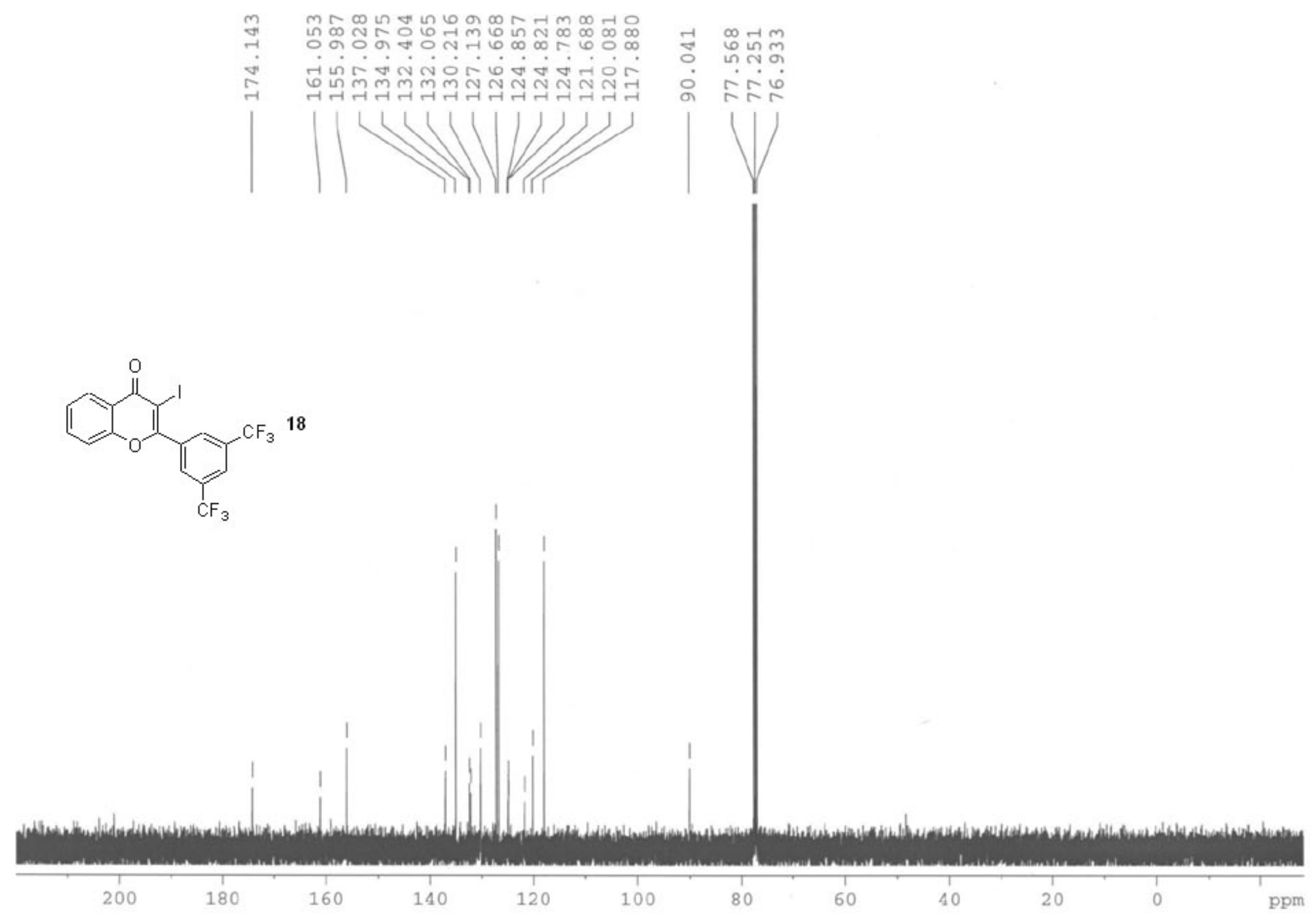


Hr

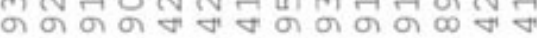
intrintribi

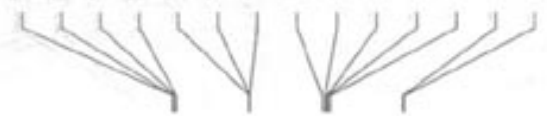

แ का

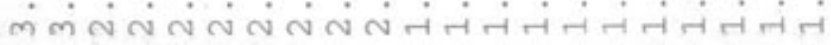
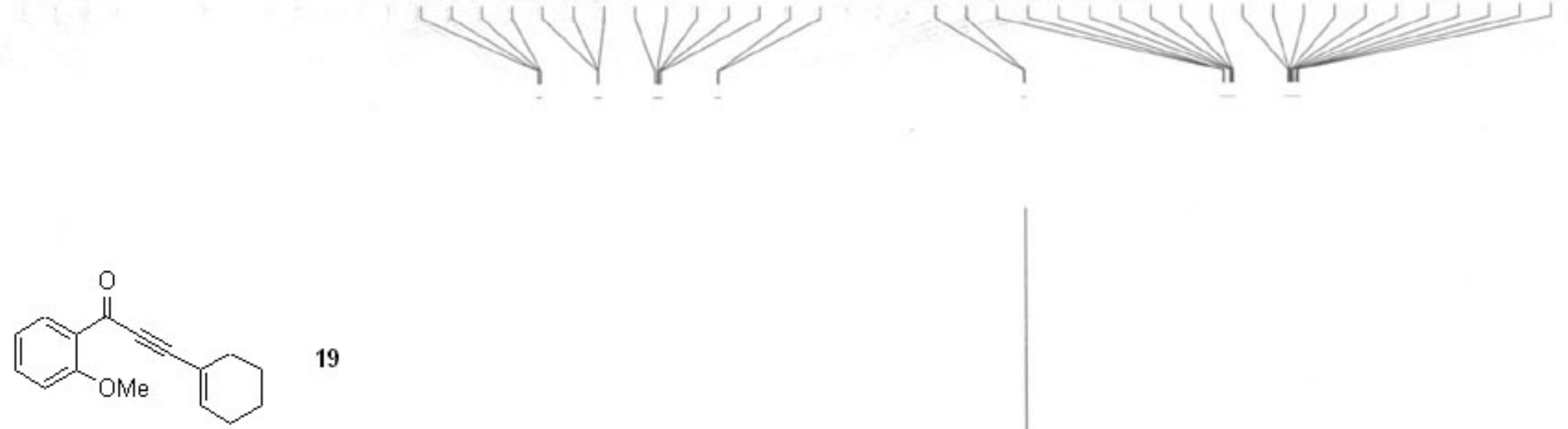

19

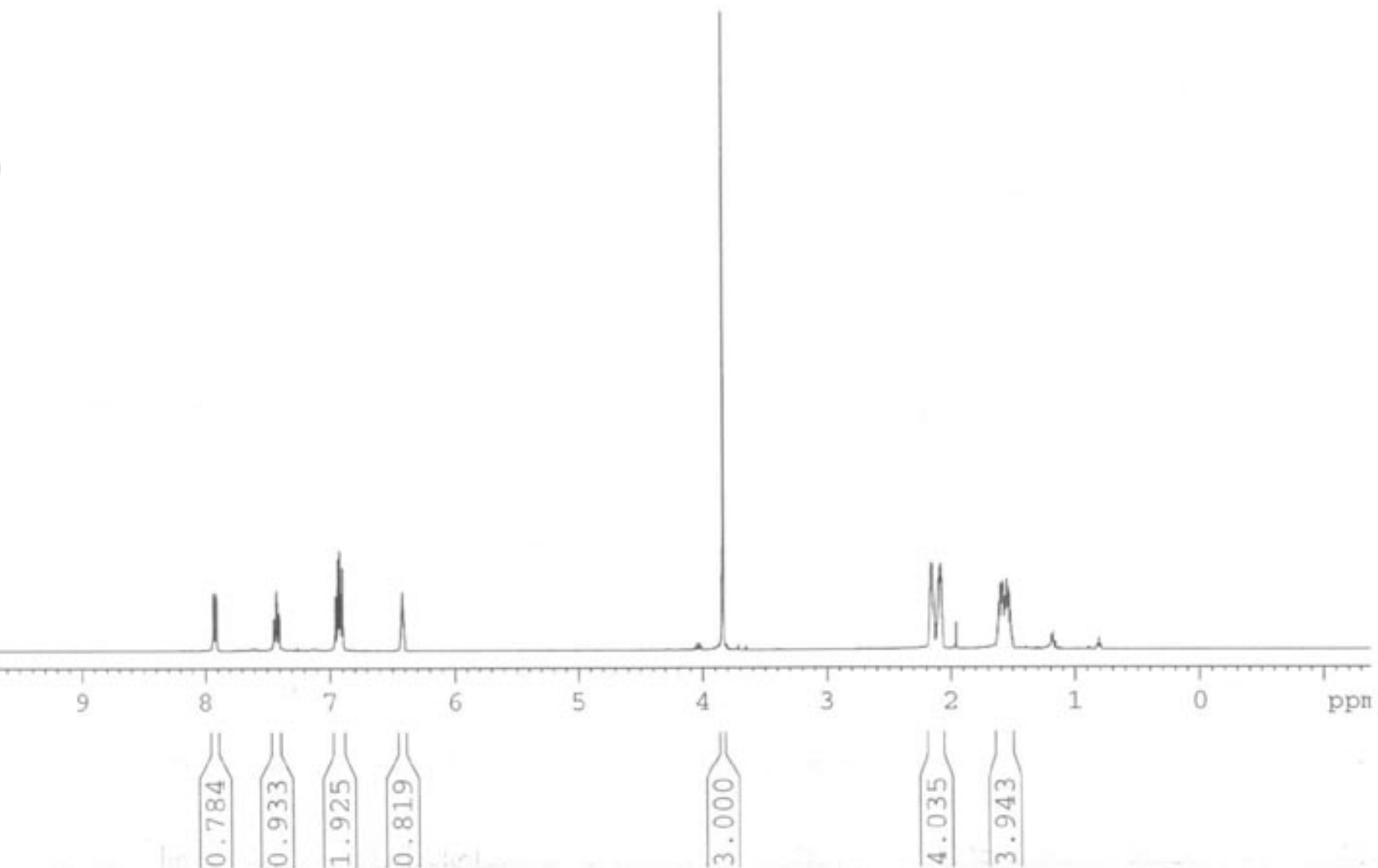

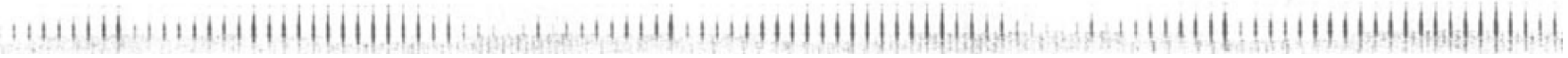



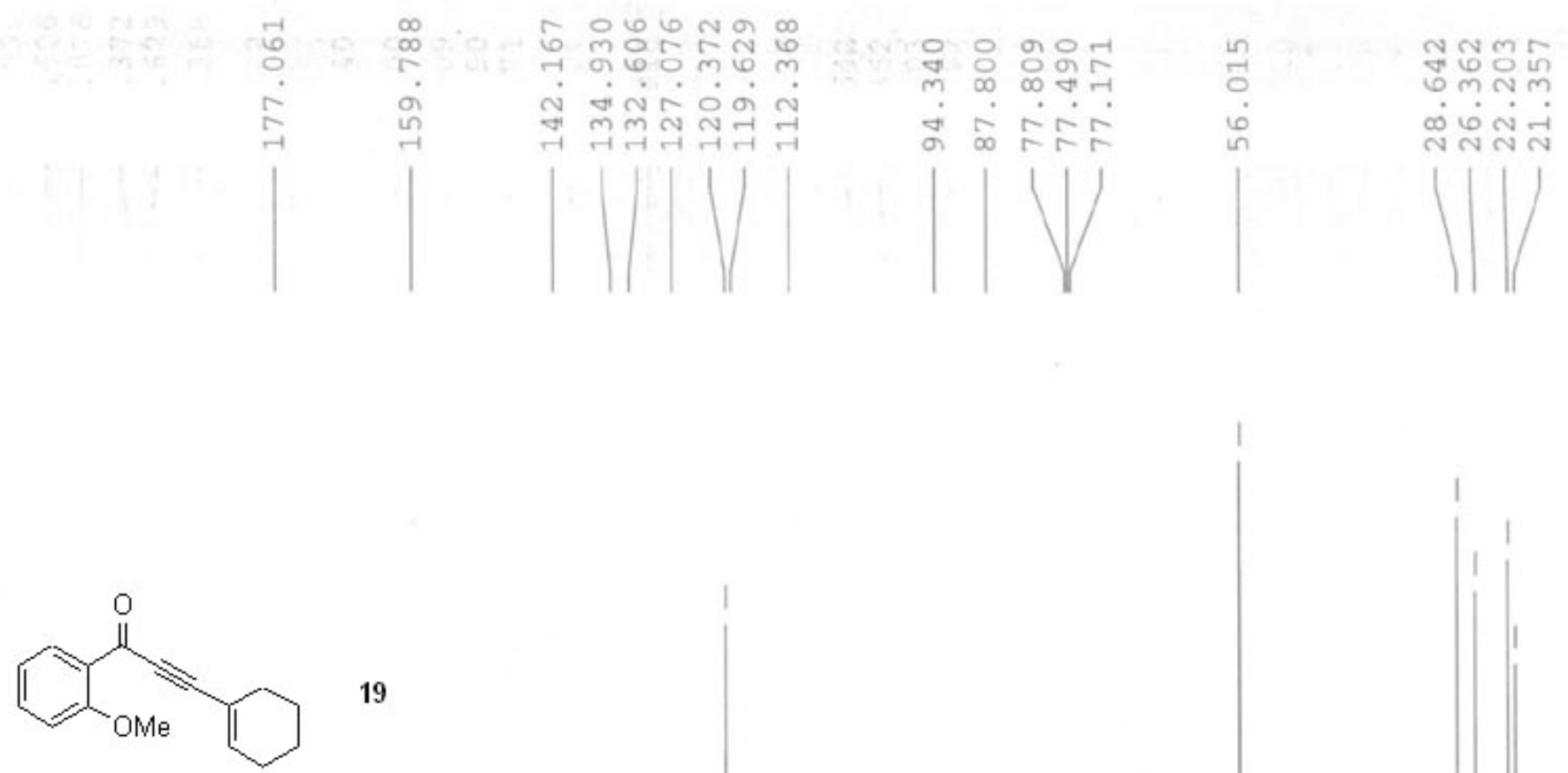

19

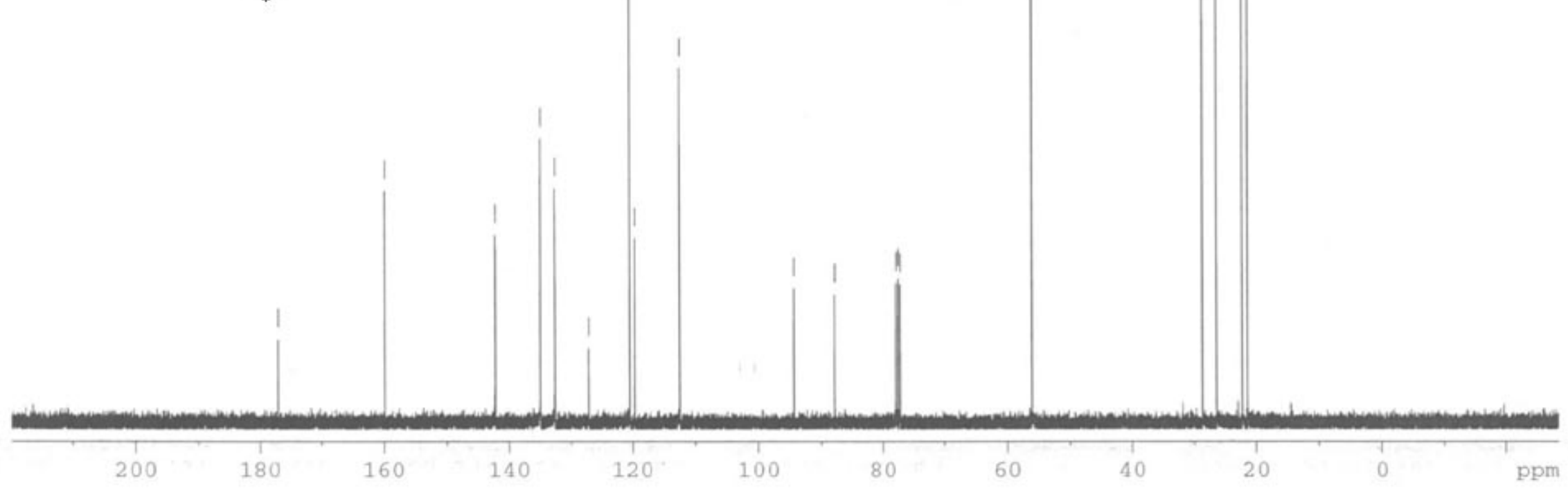

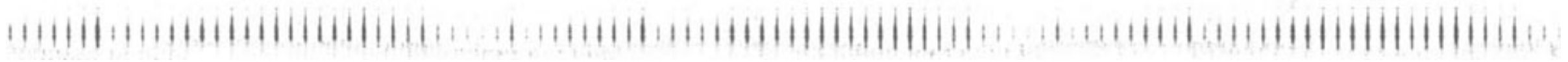


45

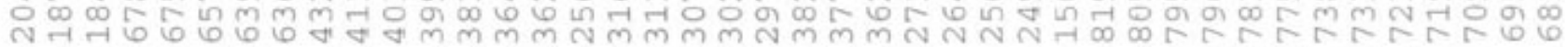

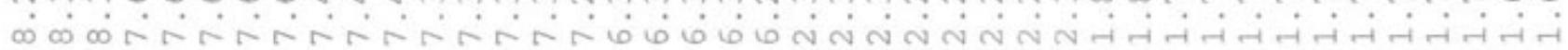<smiles>O=c1c(I)c(C2=CCCCC2)oc2ccccc12</smiles>

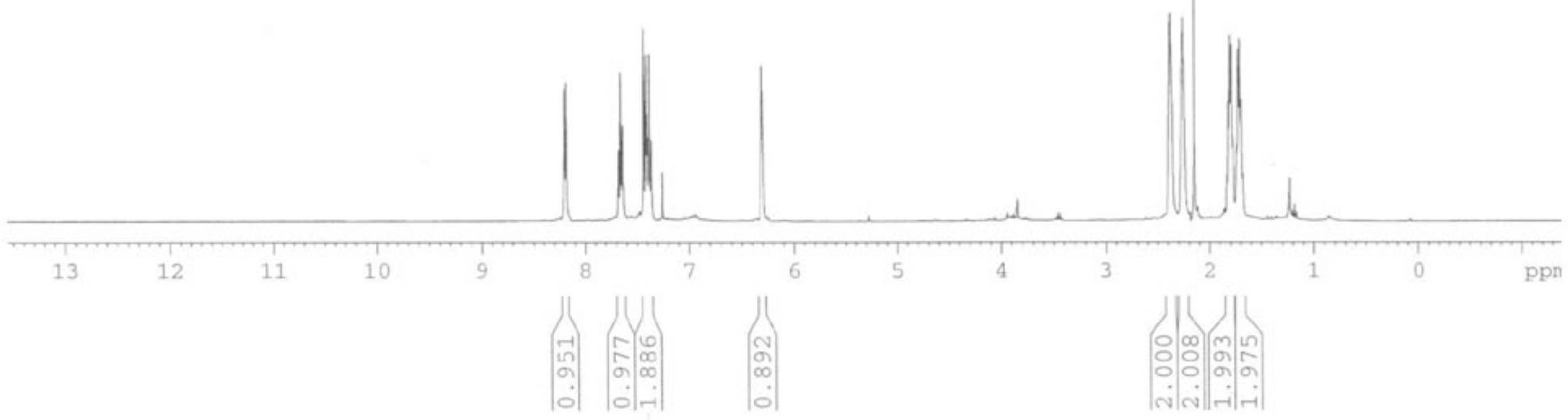

S59 

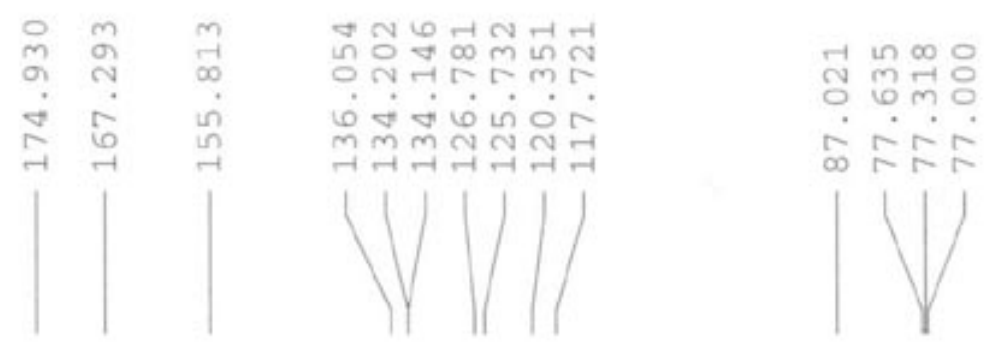

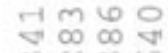

. बूल
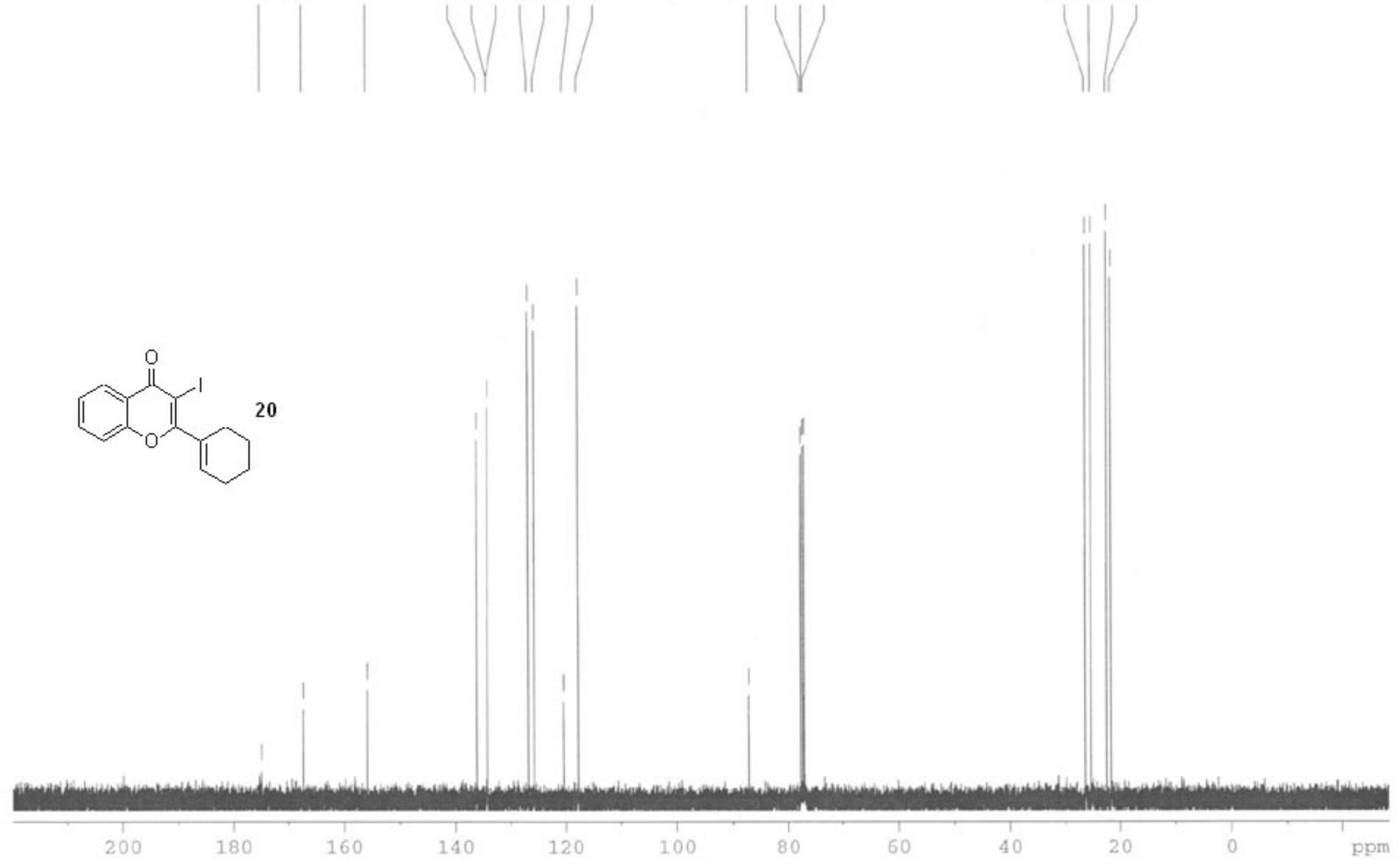

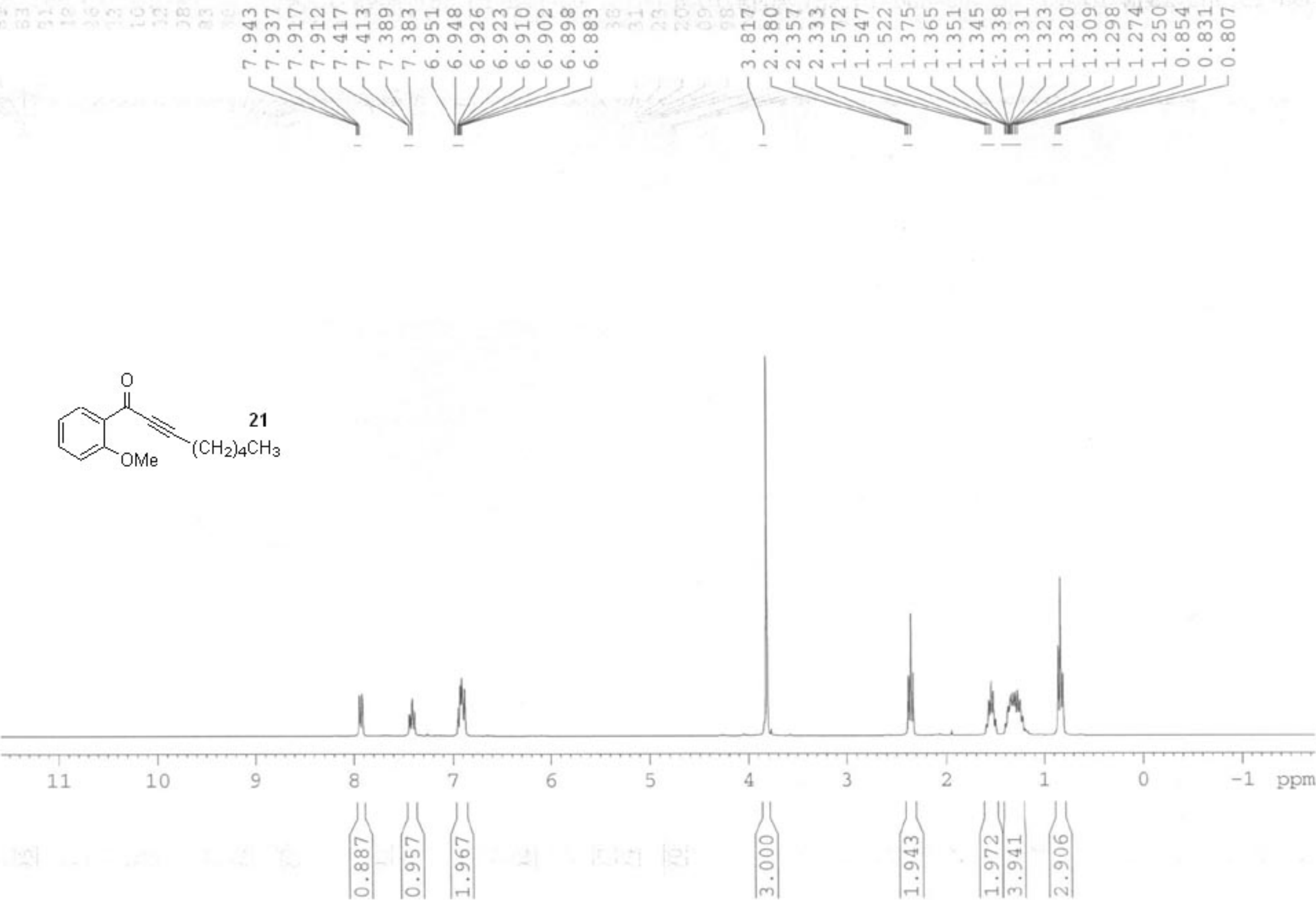


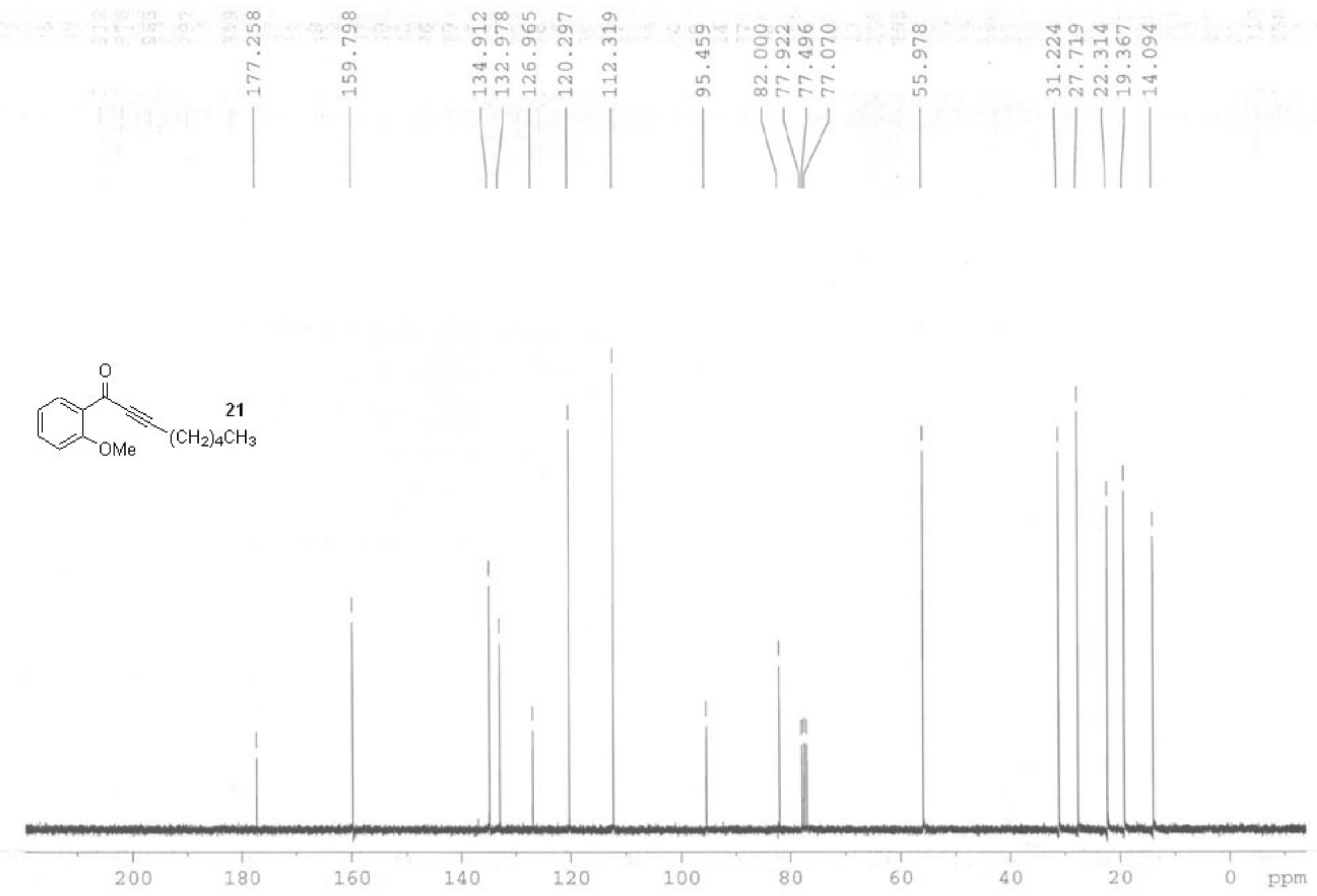


N50 공ำ

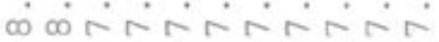

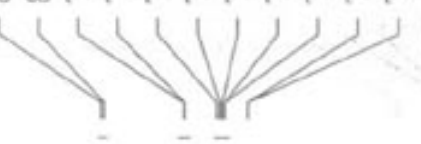

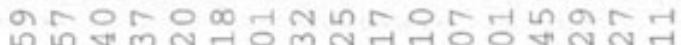

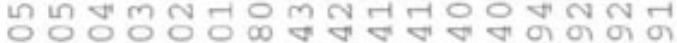

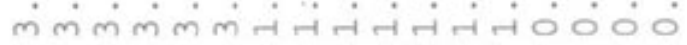

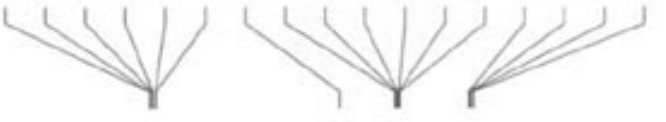

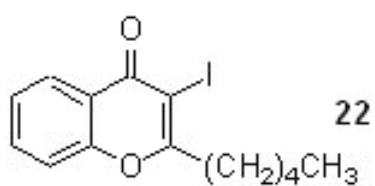

22

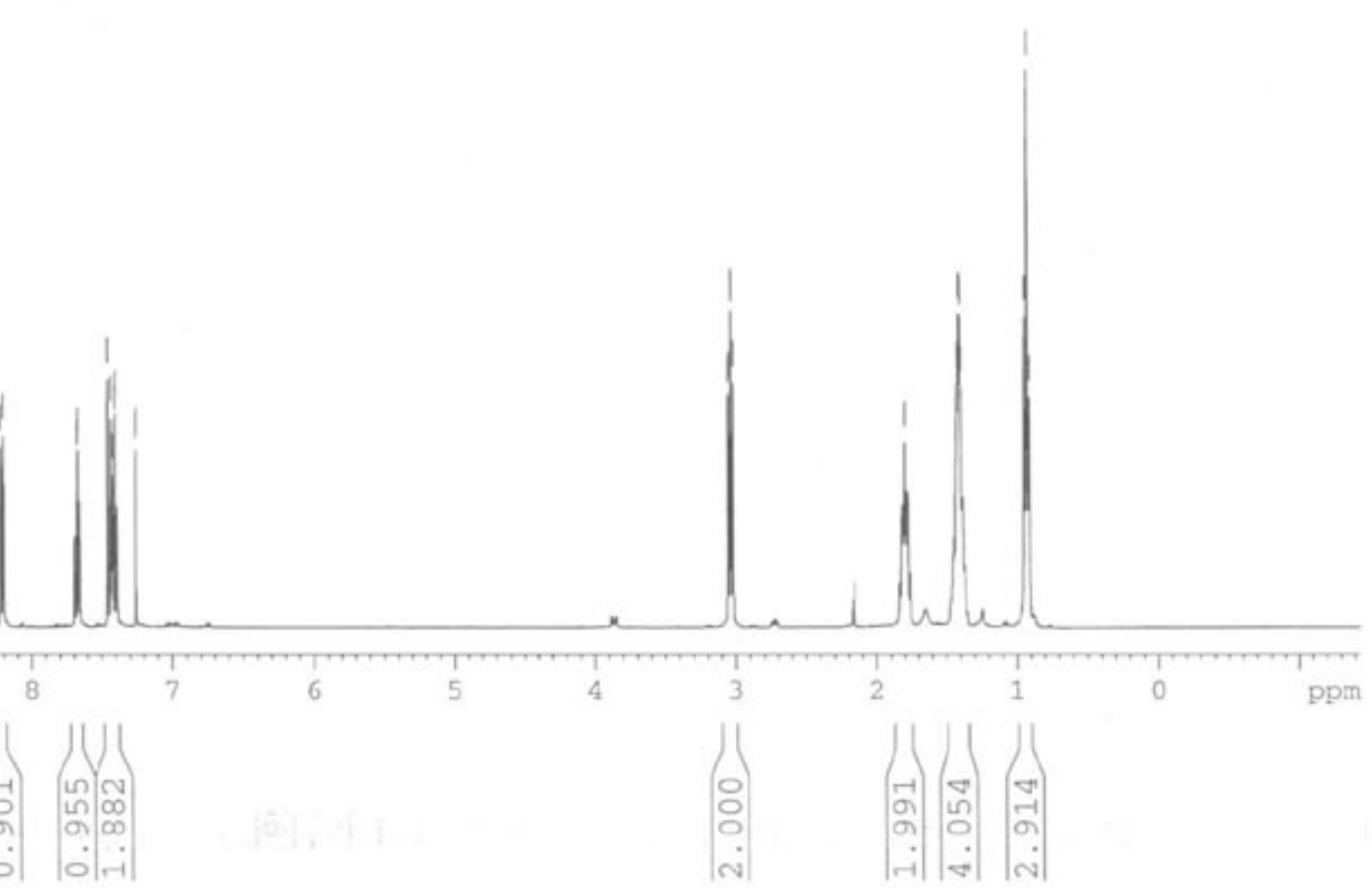



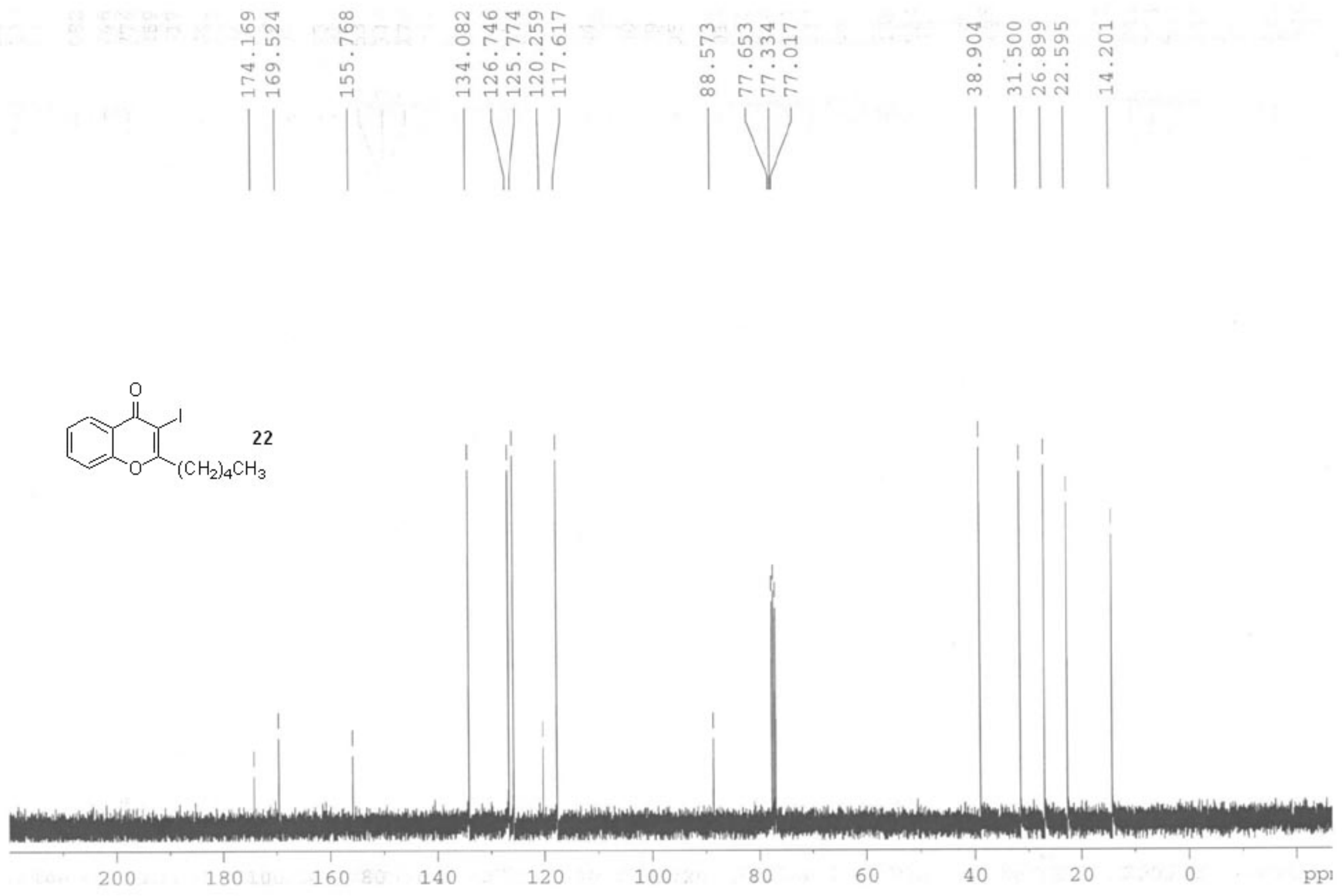
m ${ }^{\infty}$ \%

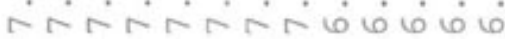

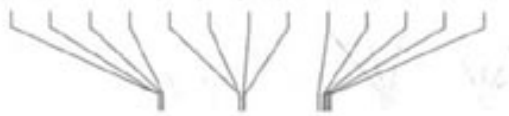

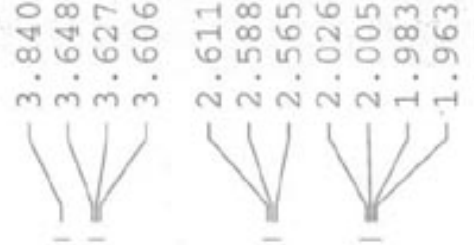

23

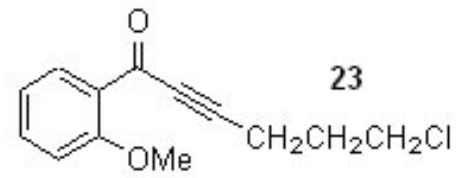

mmm

$1 V 1 / 11$
ปี่

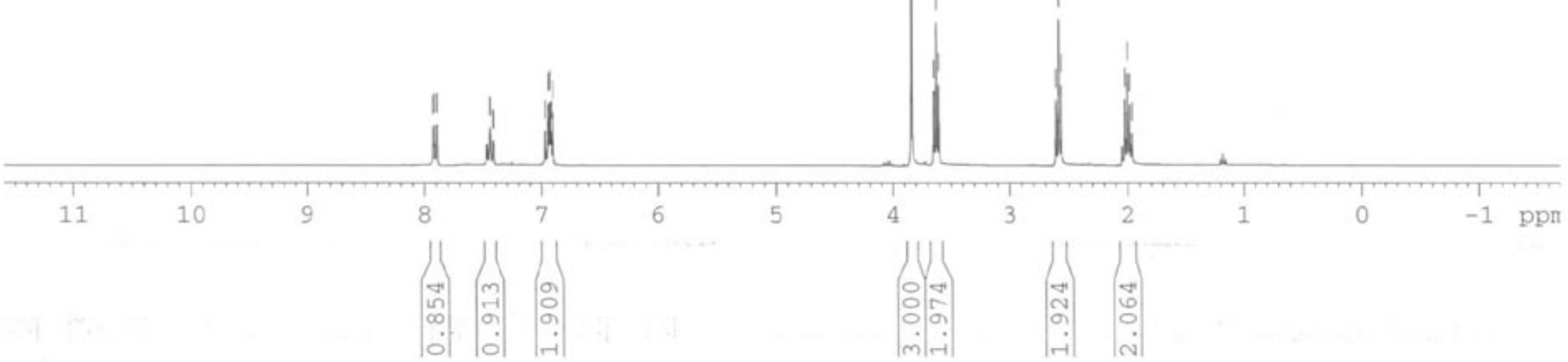



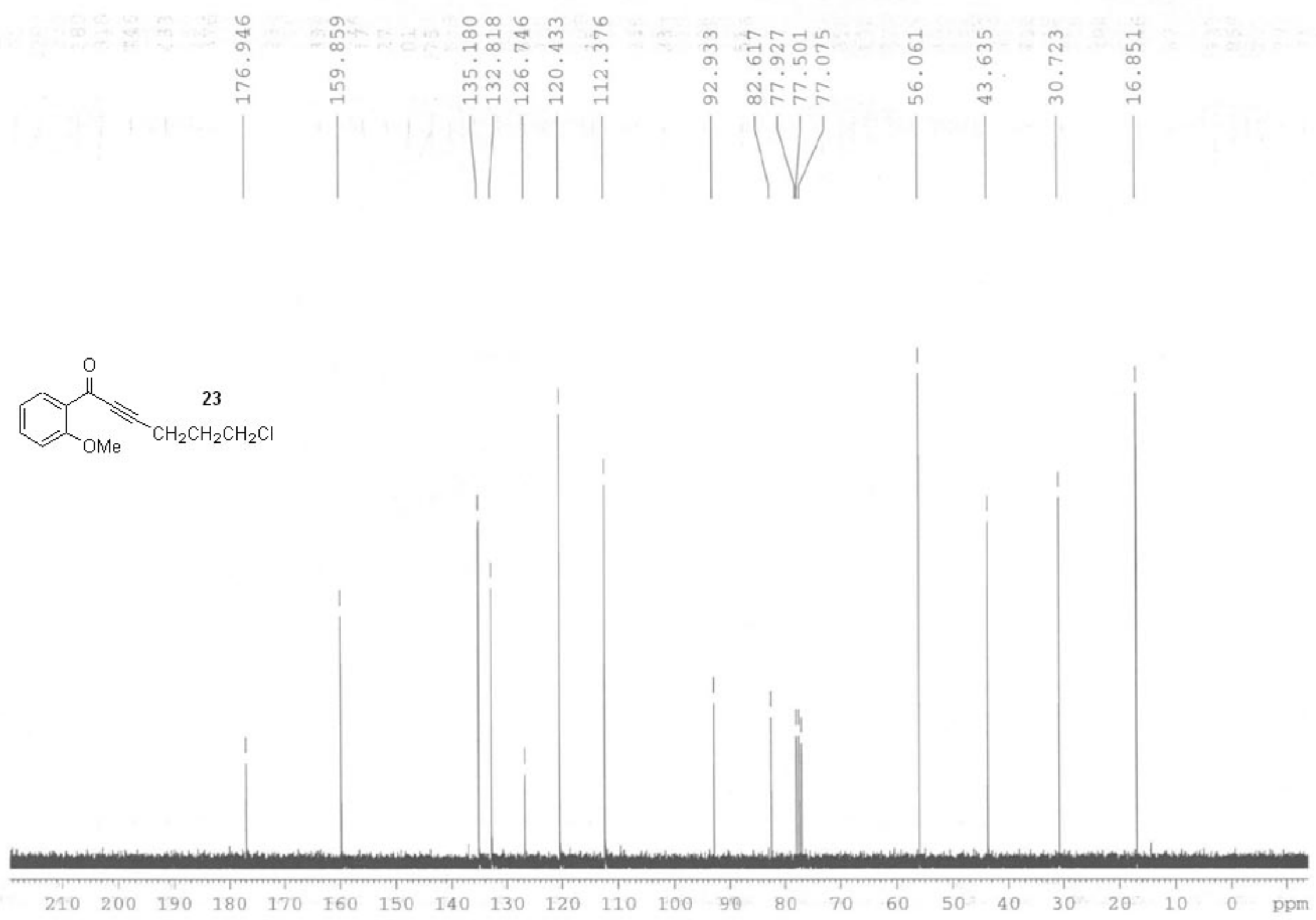


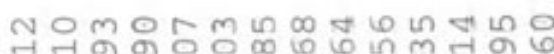

ง

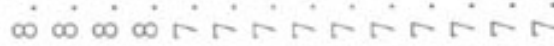

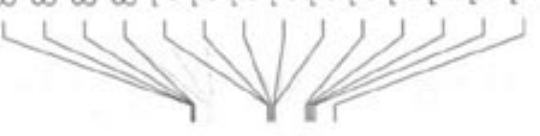

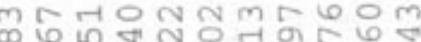

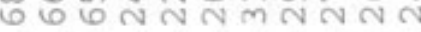

लंmmंm $N$ N

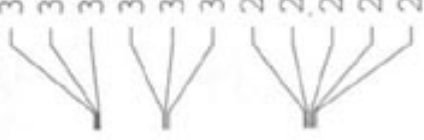

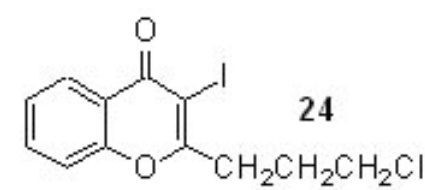

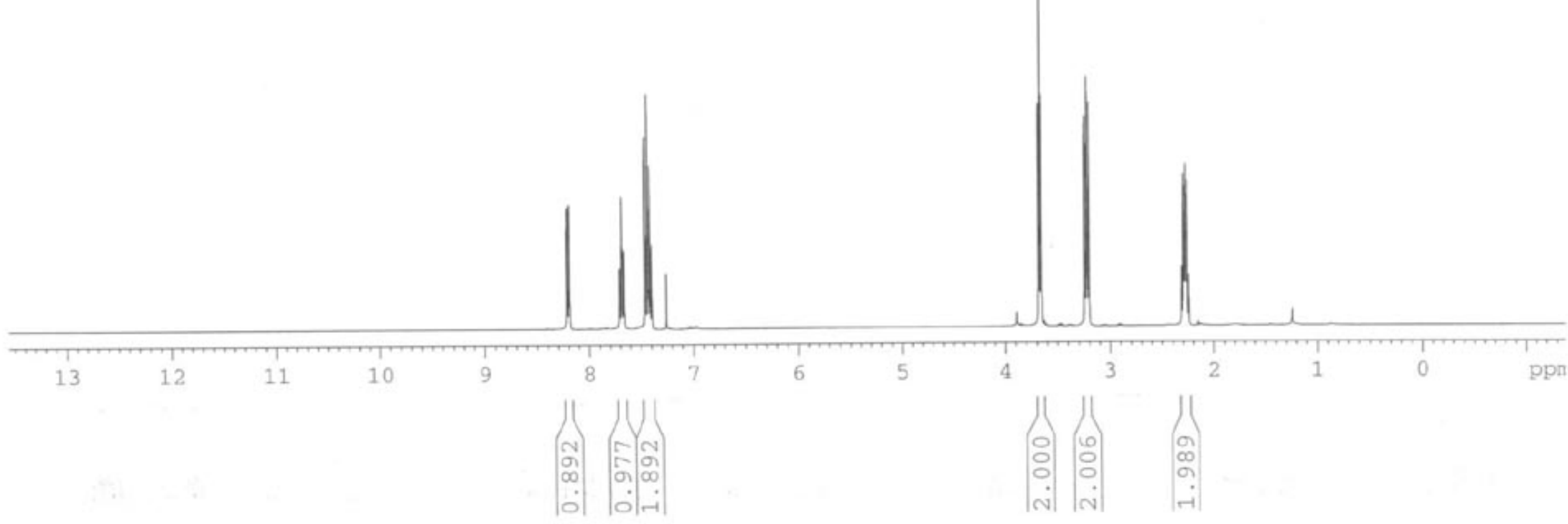



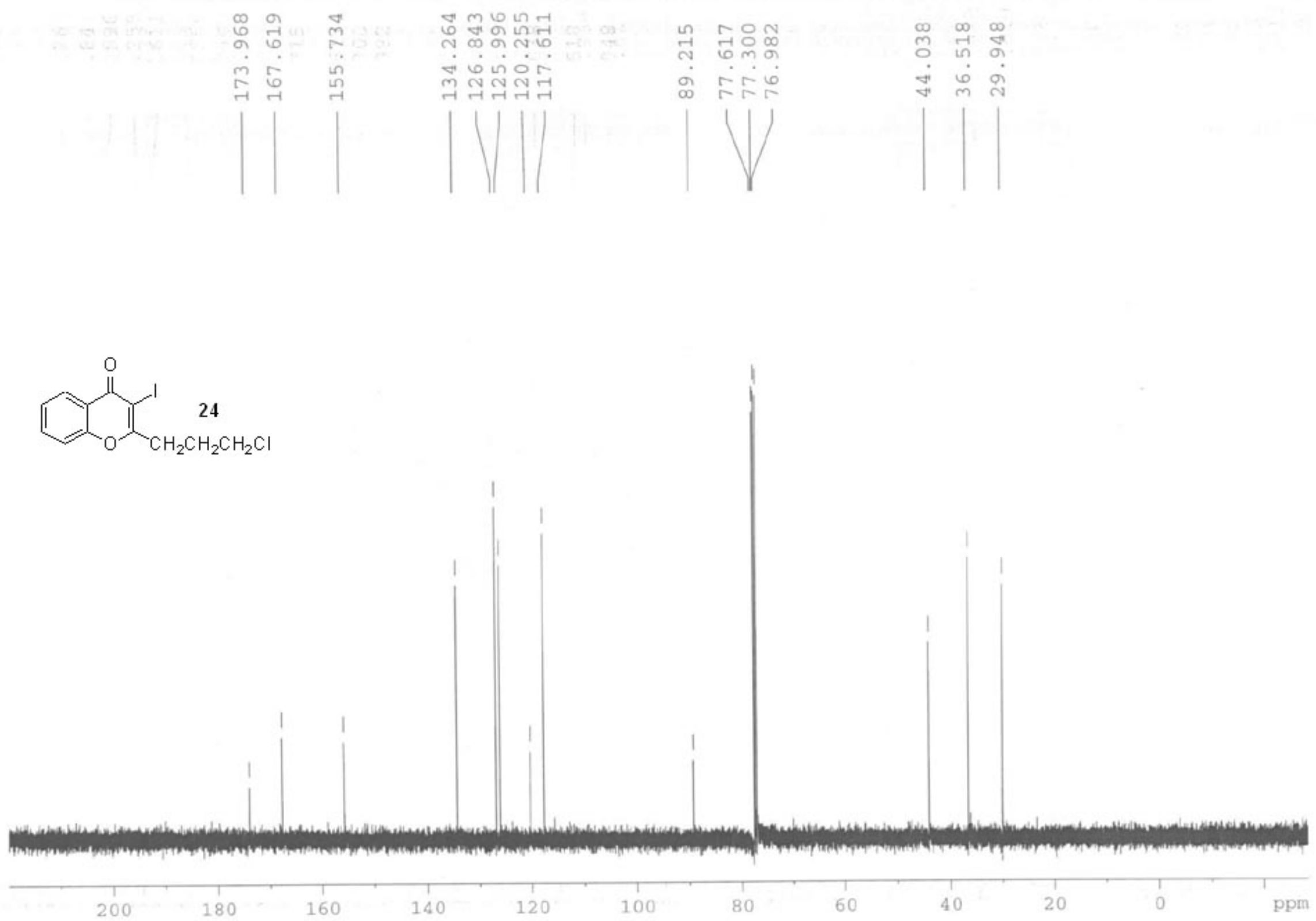

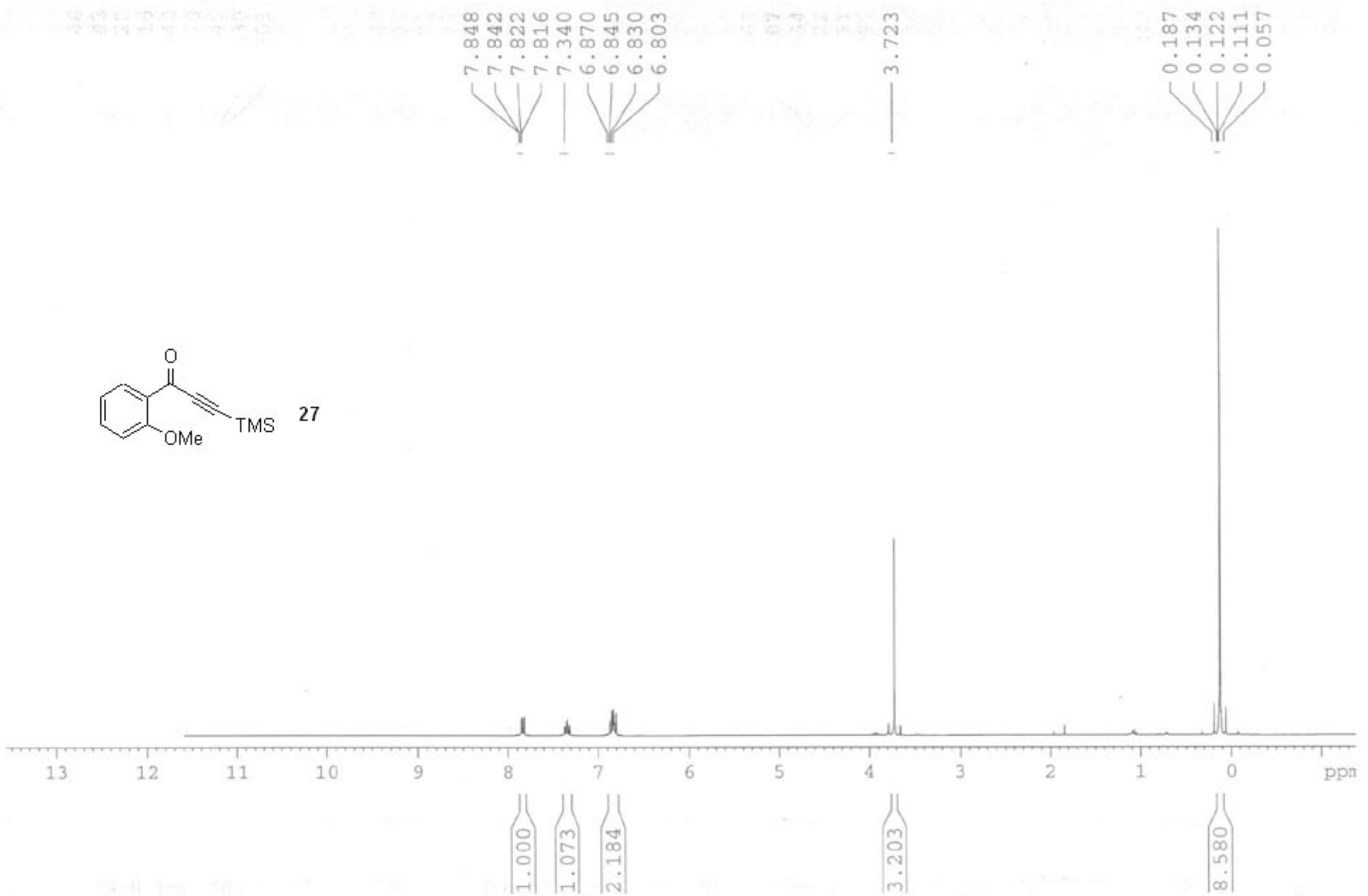

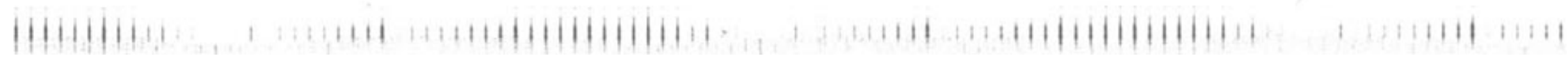



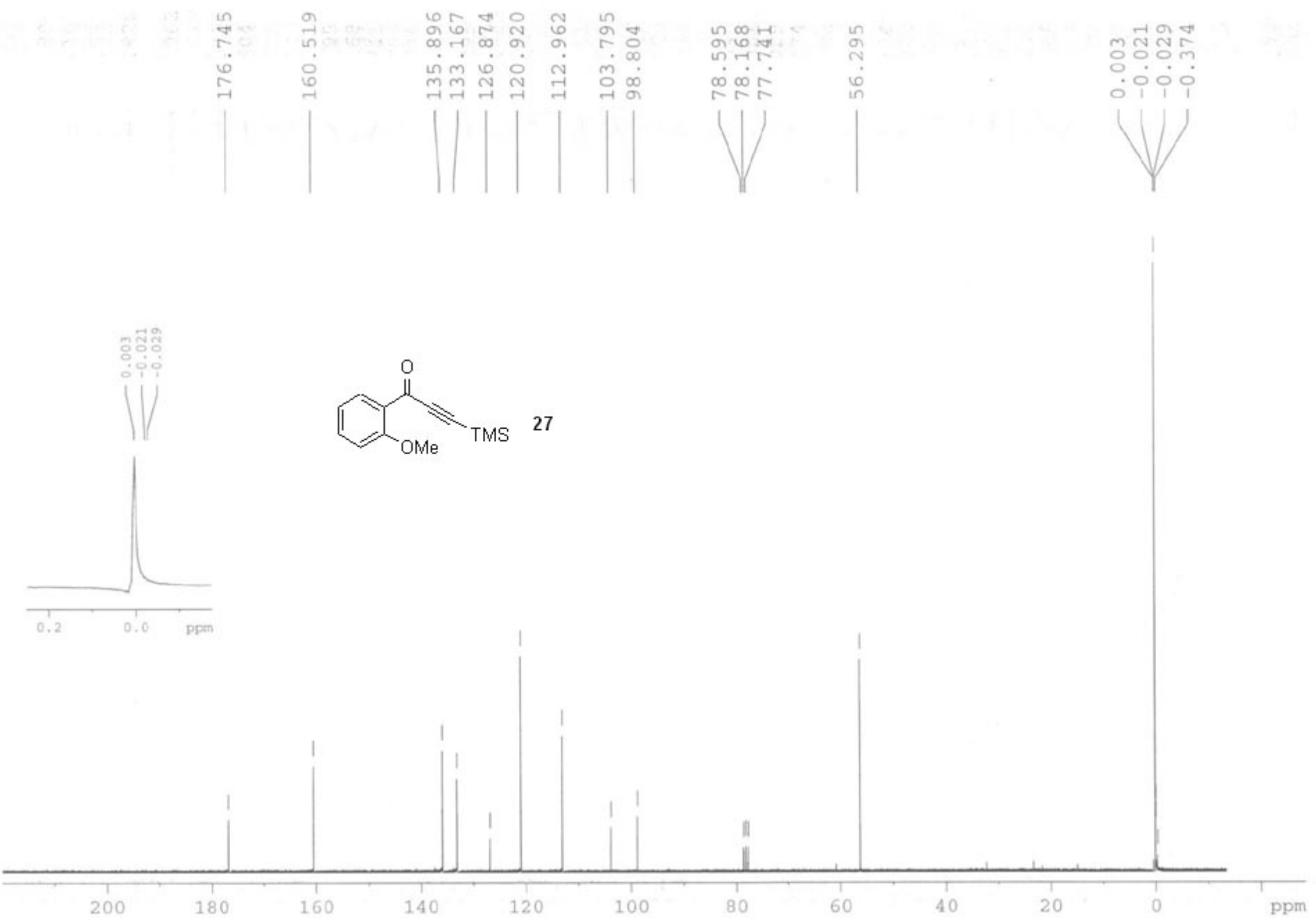

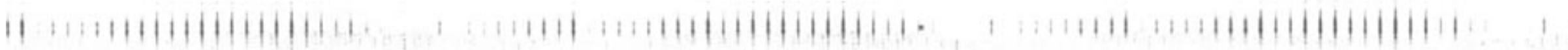



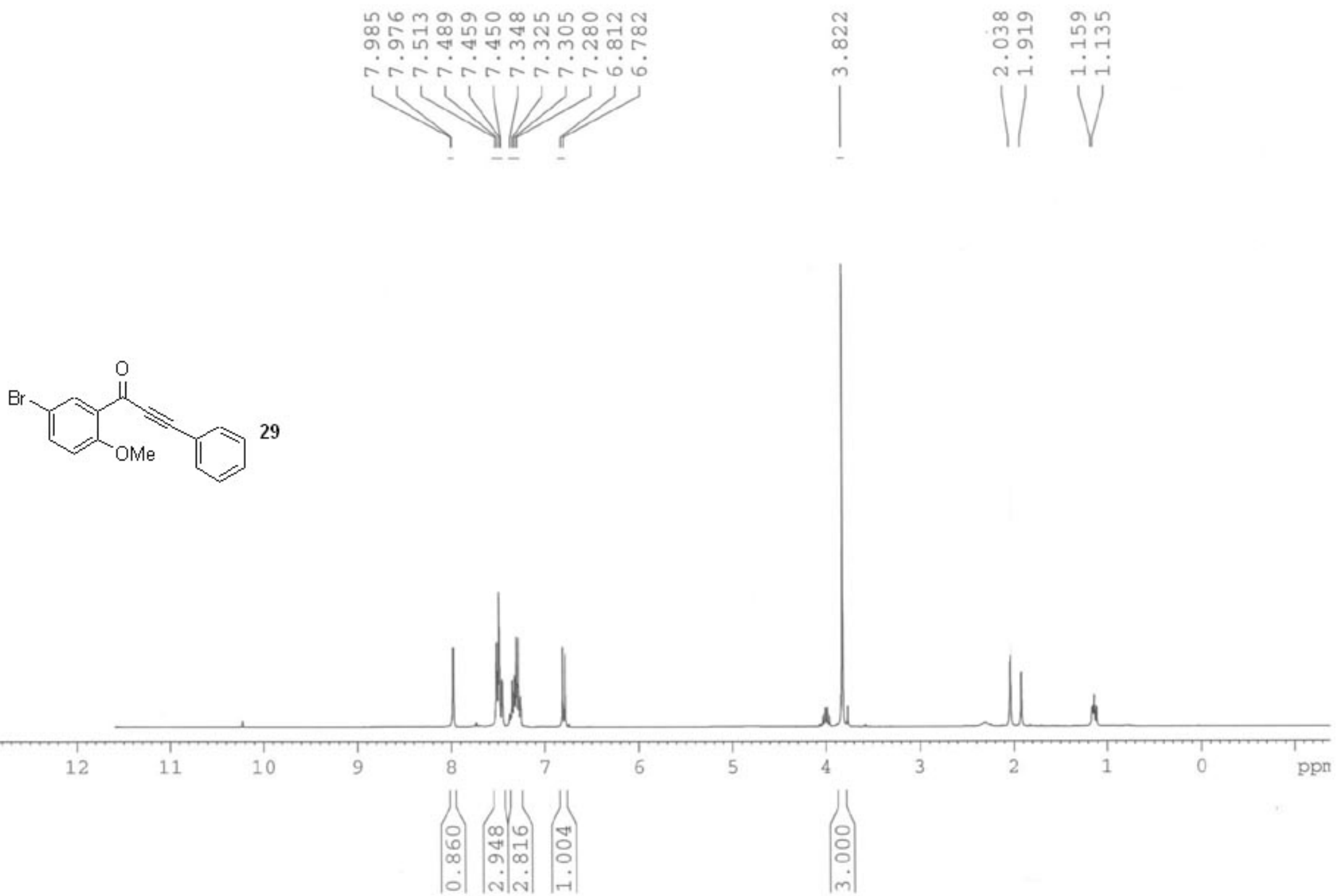

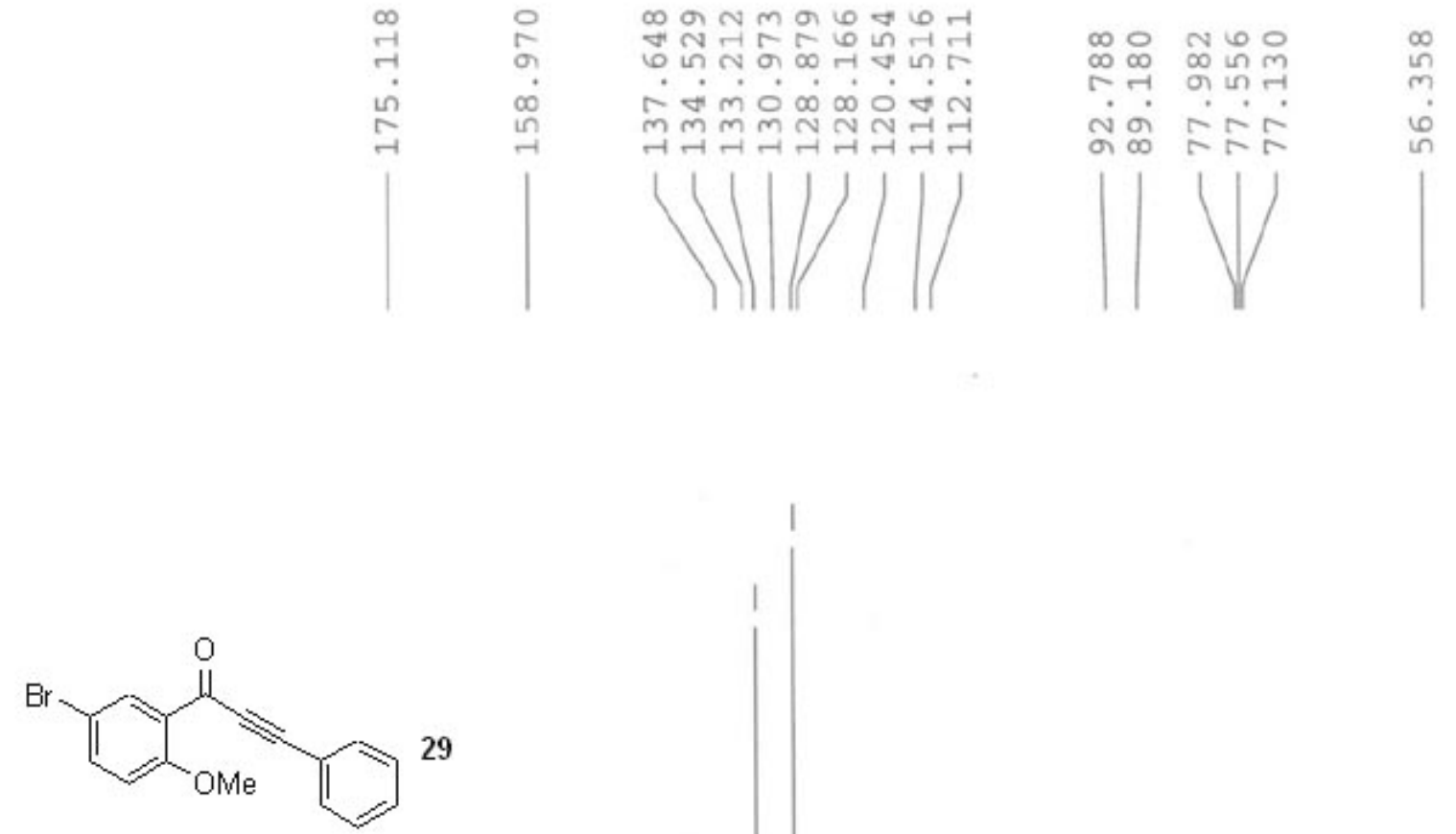

$$
9
$$

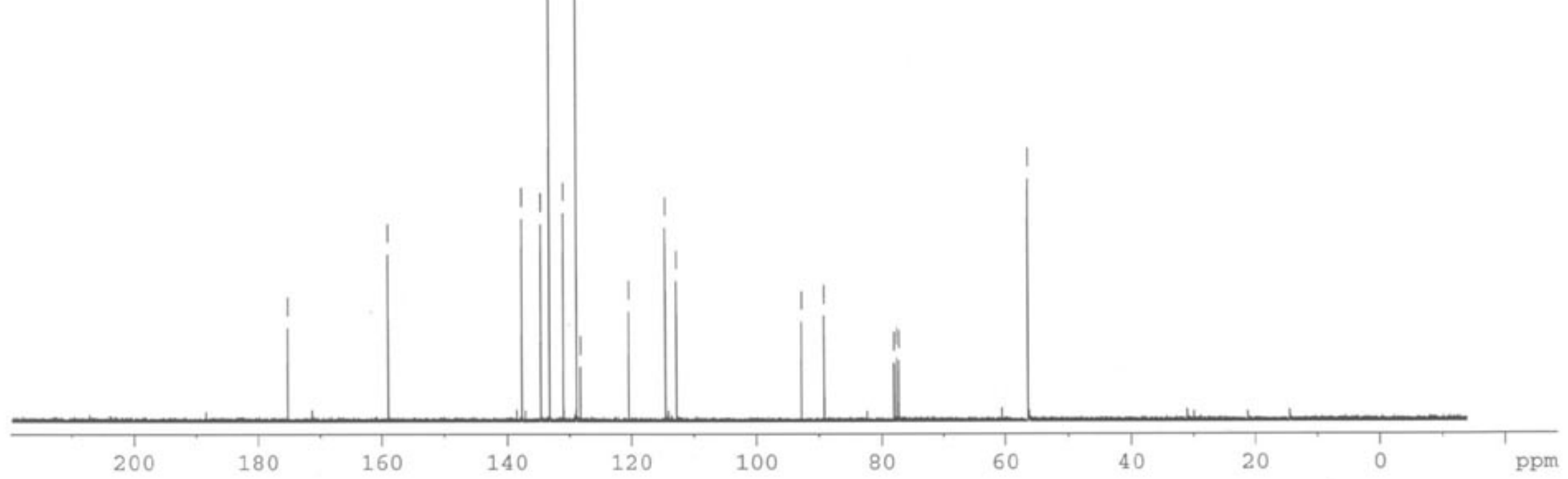




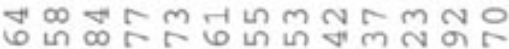

m

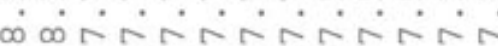
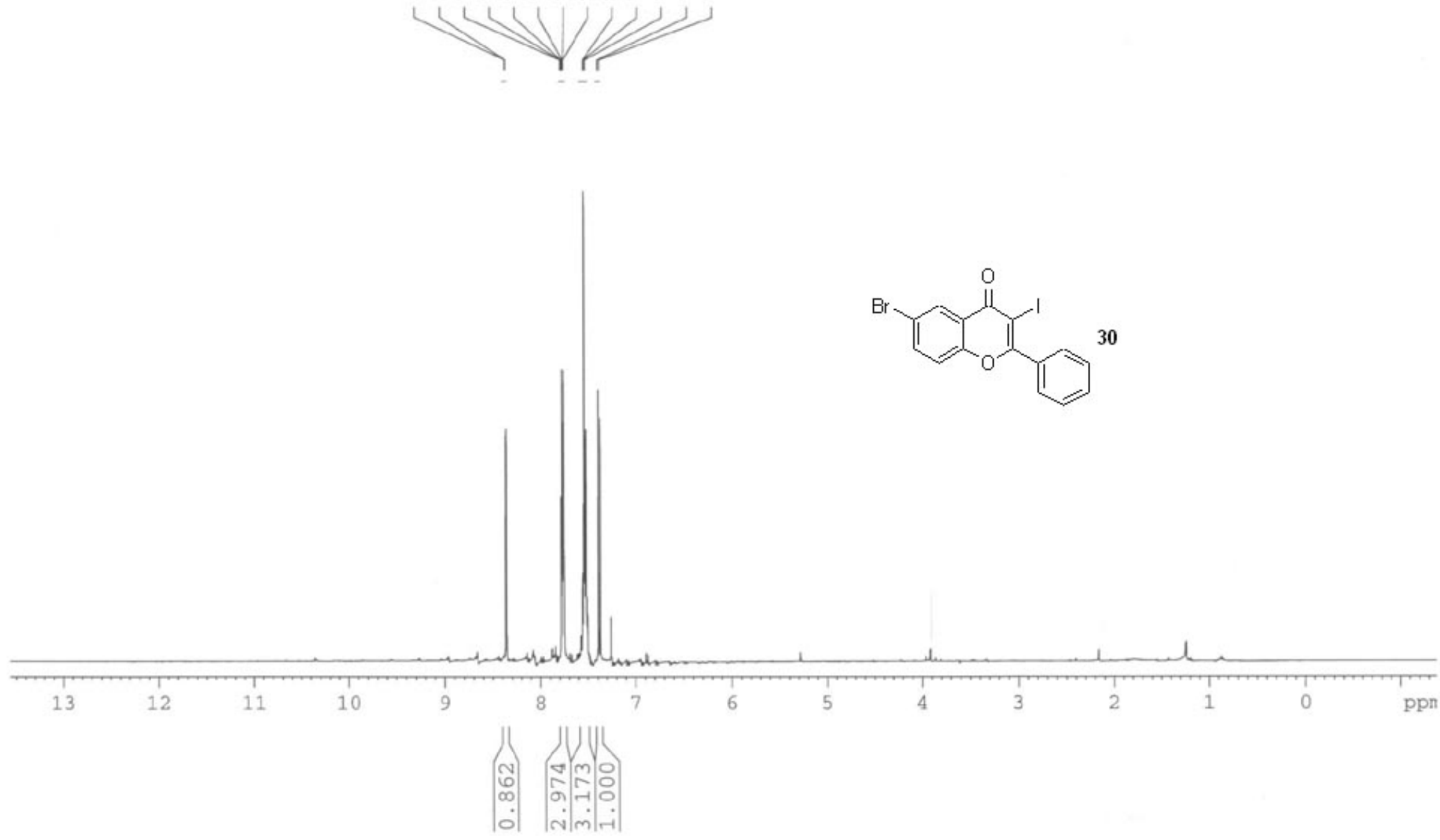

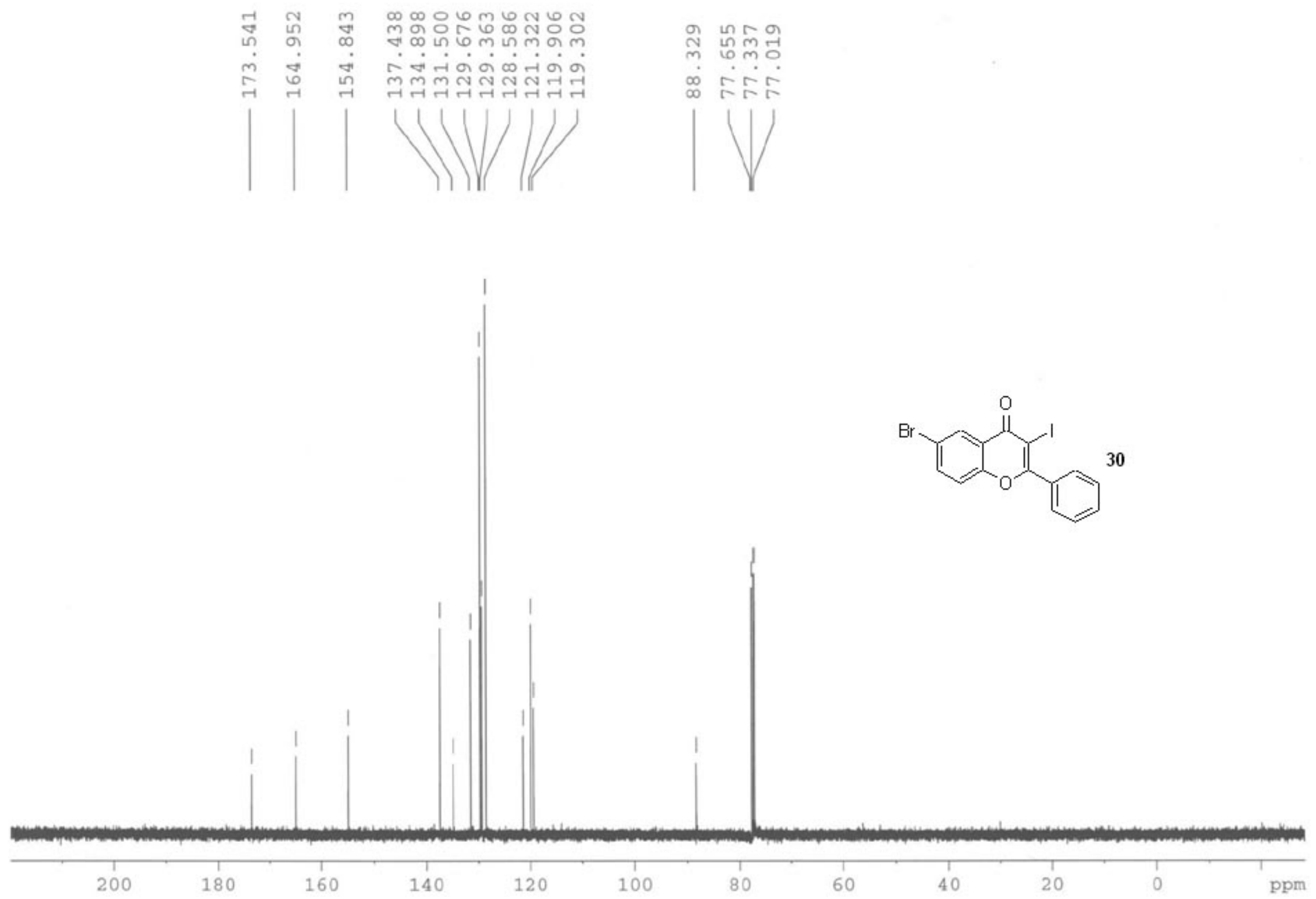

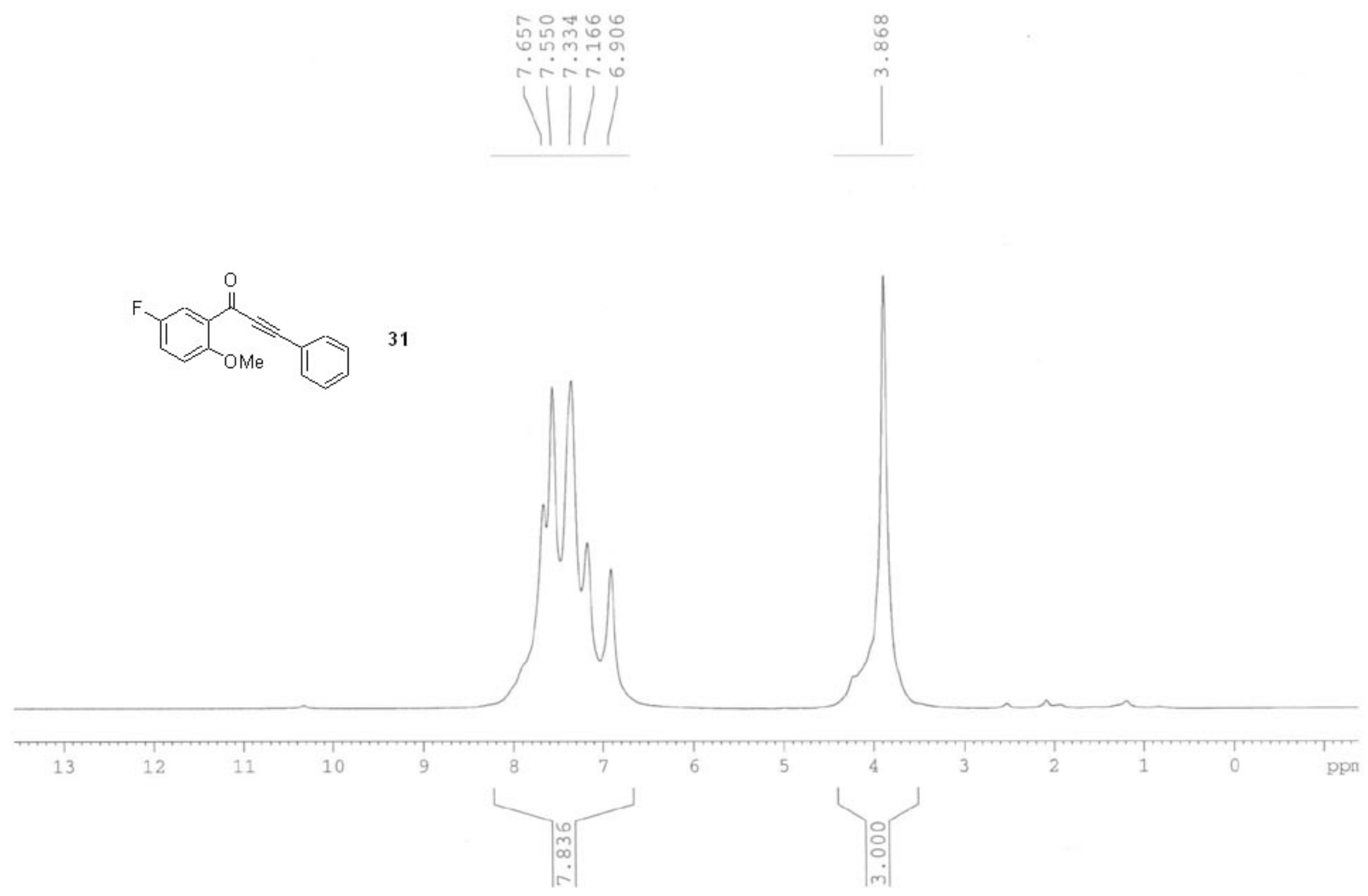


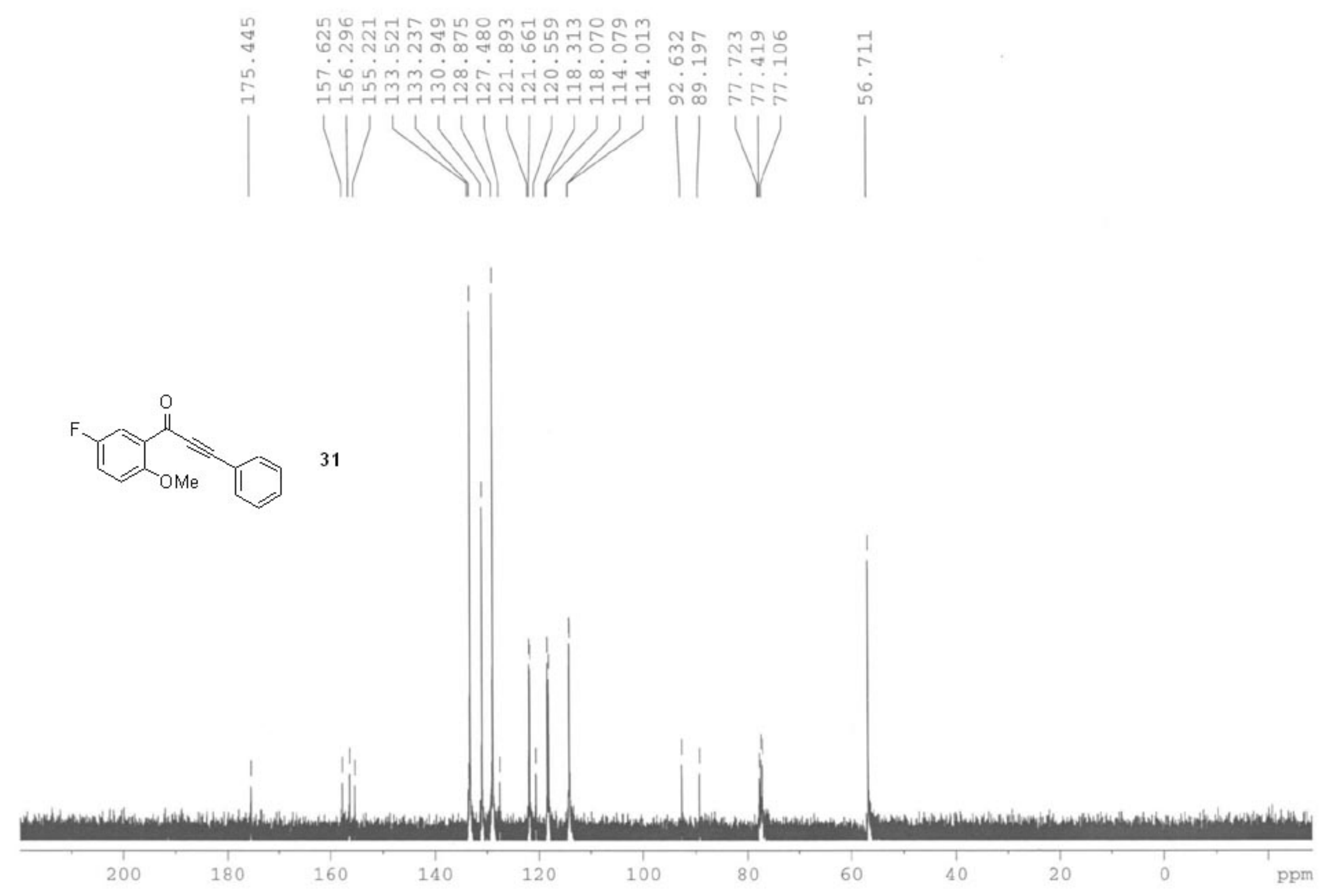


ผ ㄴํำ

$\infty \begin{aligned} & \infty \\ & \infty\end{aligned} \infty$

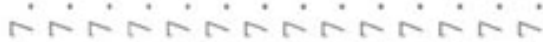

M

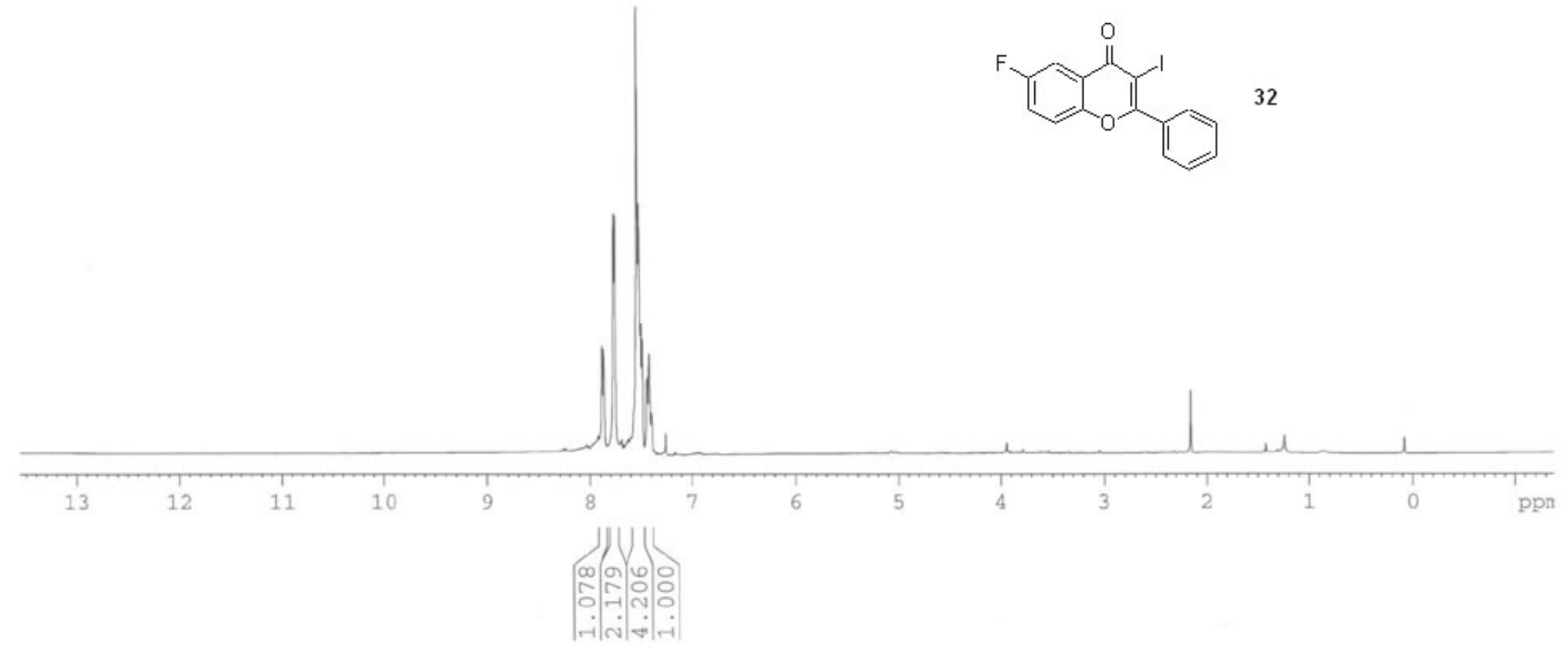


곡윤

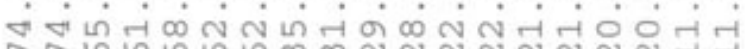

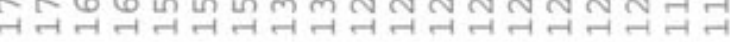

or $m \Vdash r$

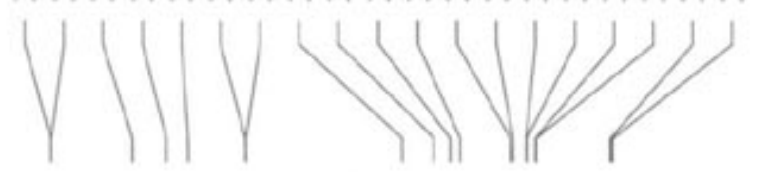

$\therefore r i r$

mith
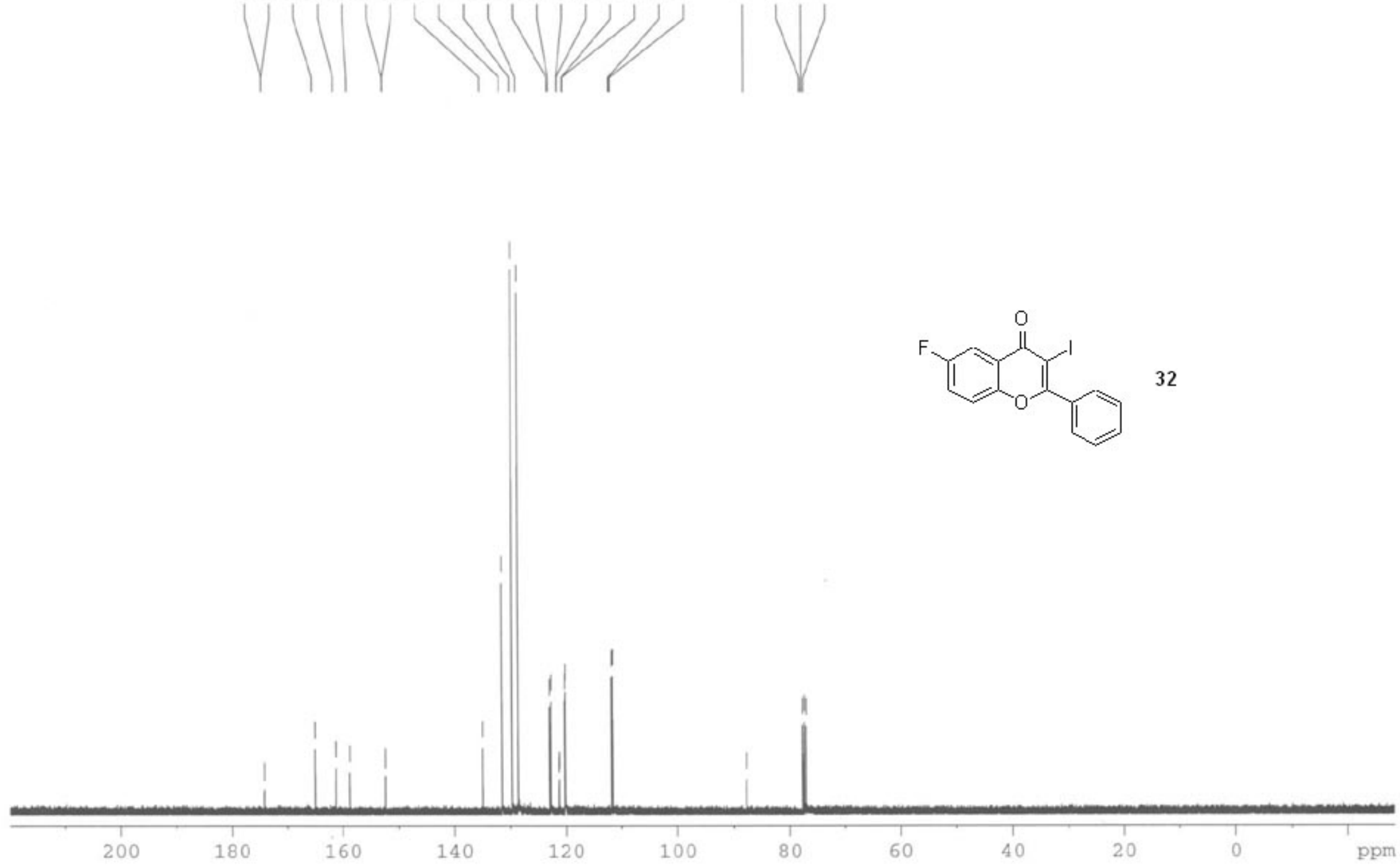

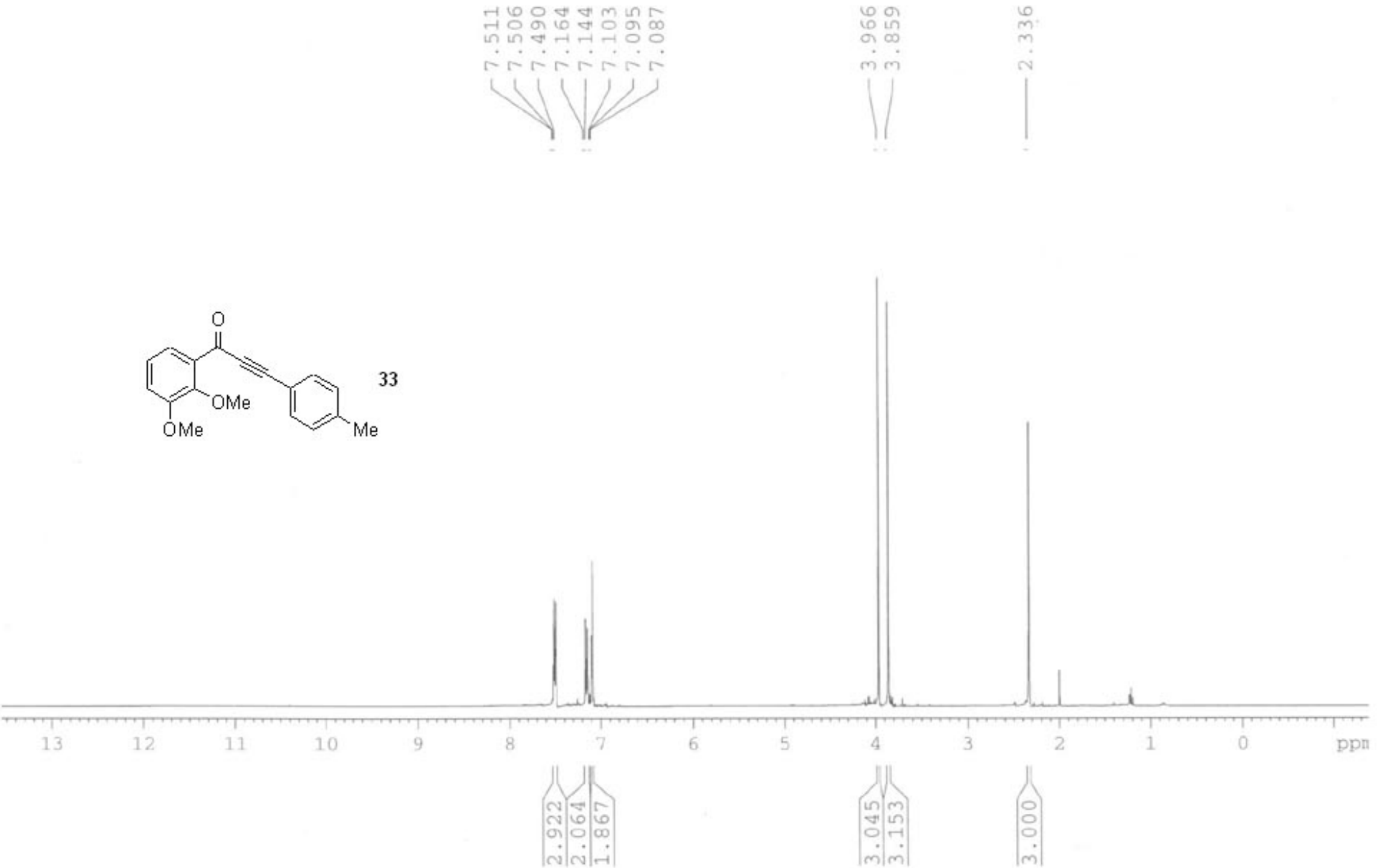


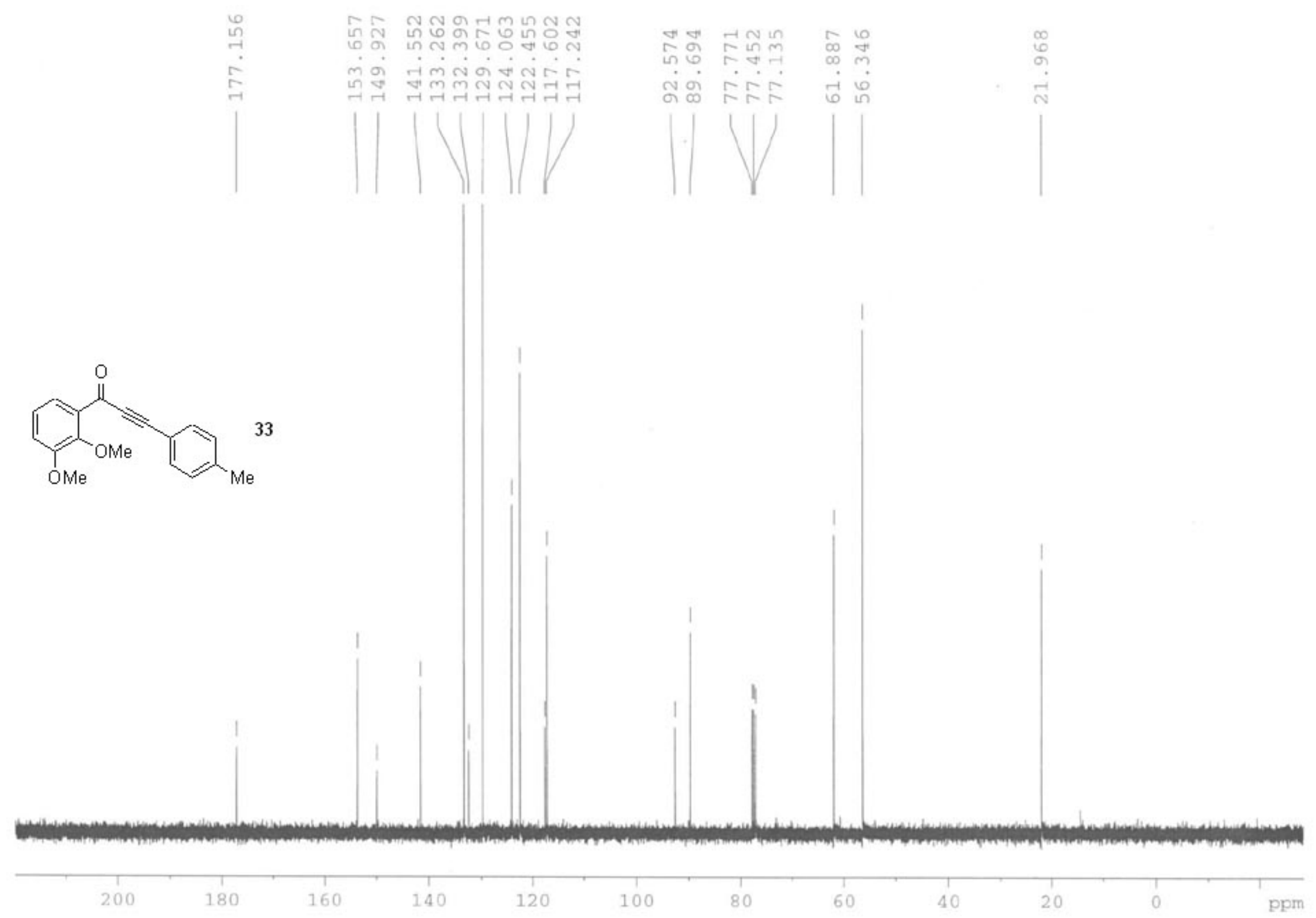




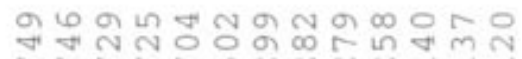

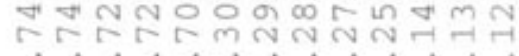

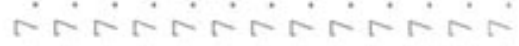

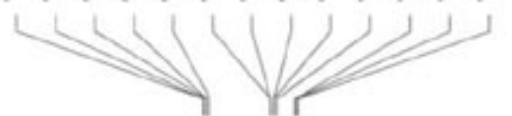
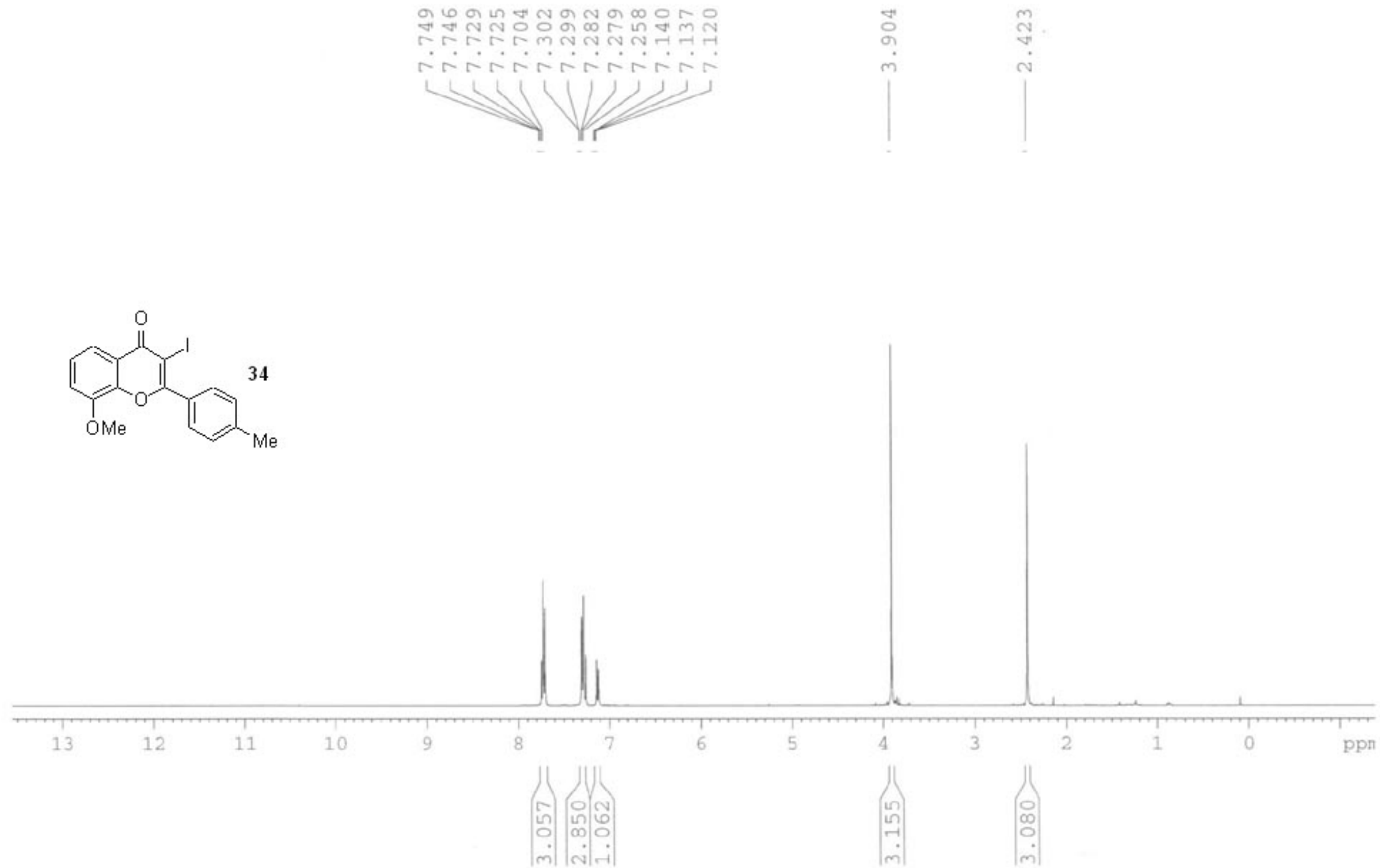

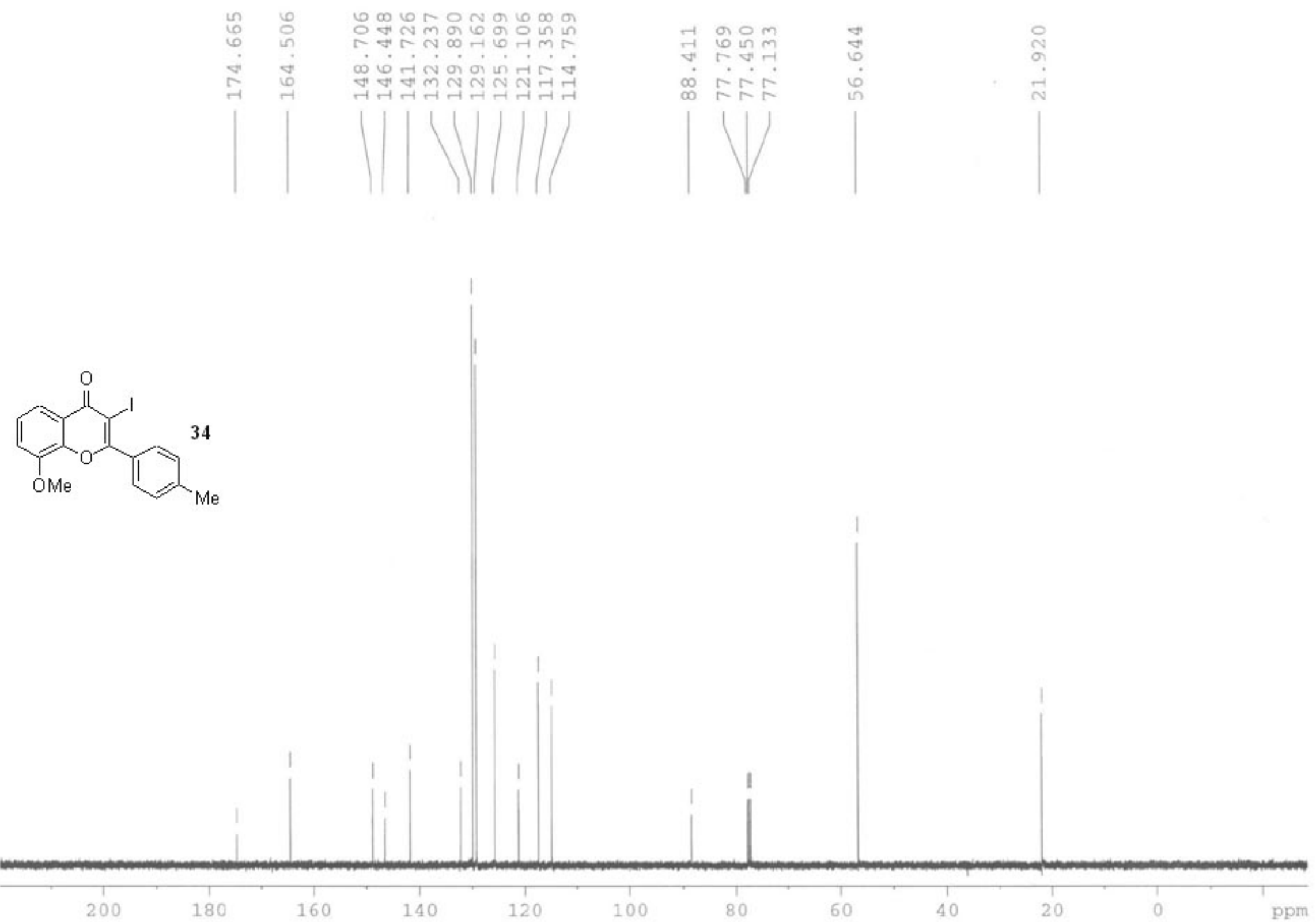


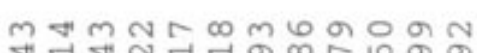

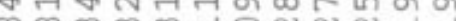

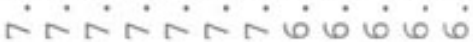

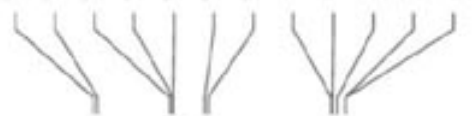

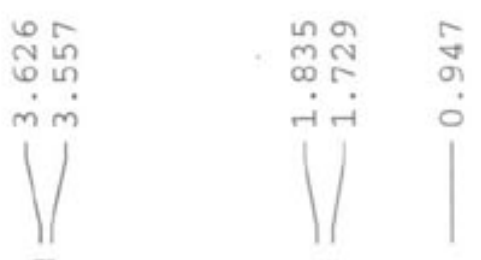
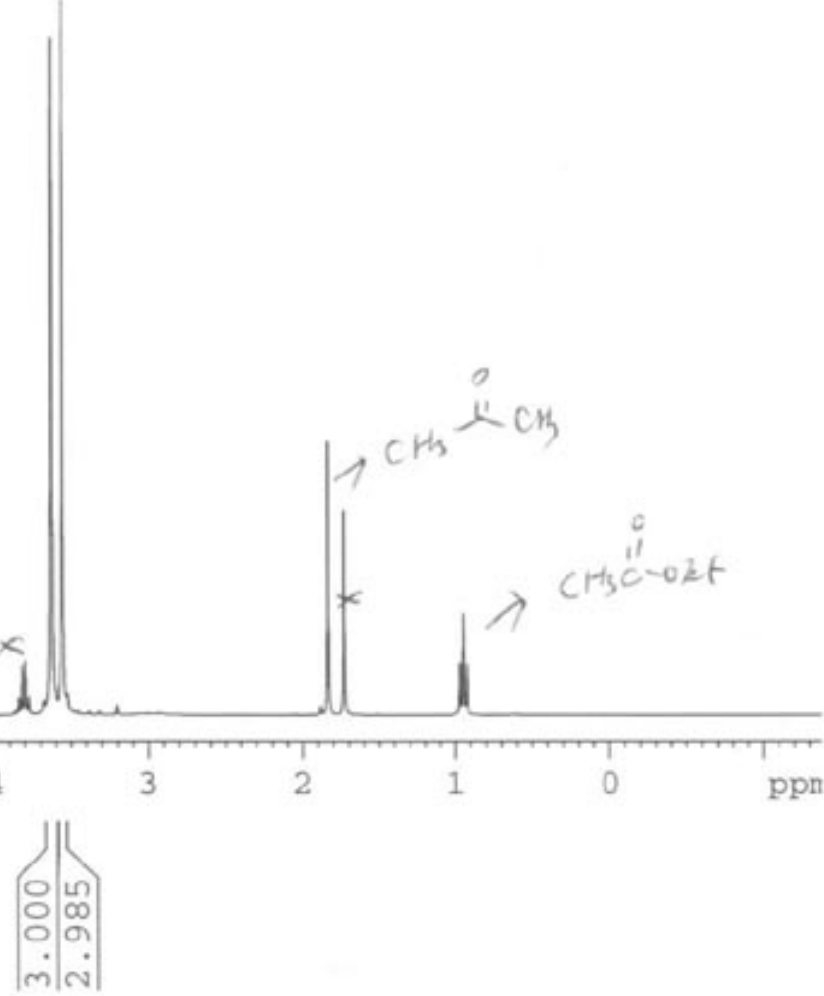

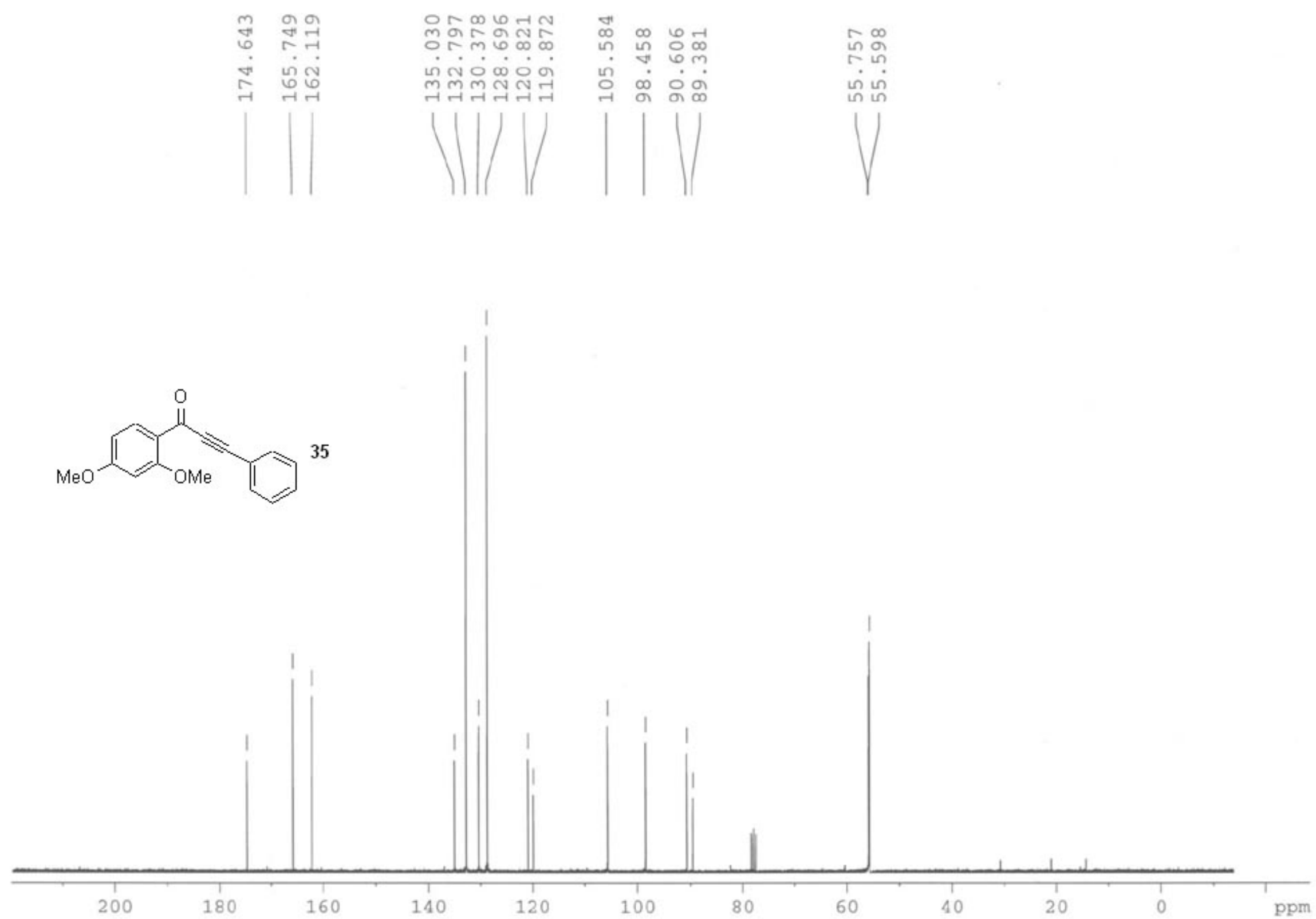


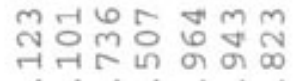

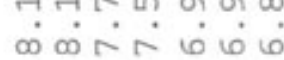

$1 / 111$

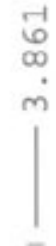

(16)

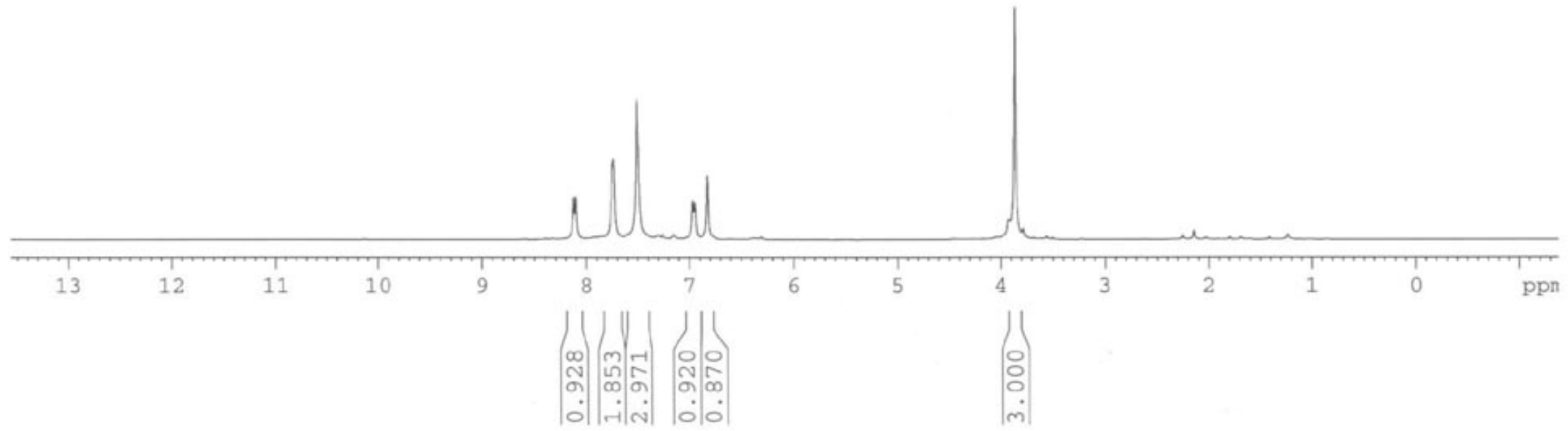



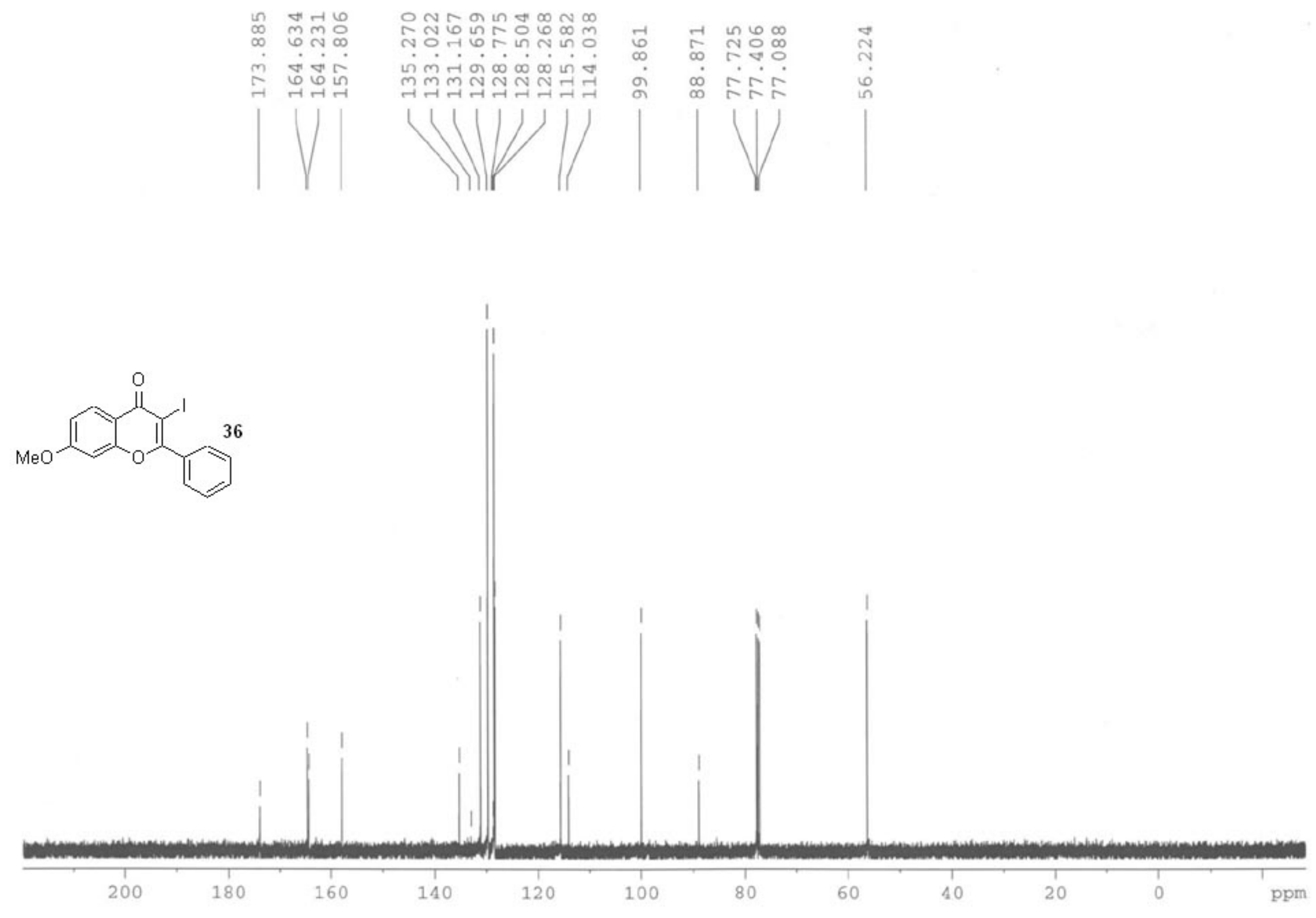

S86 

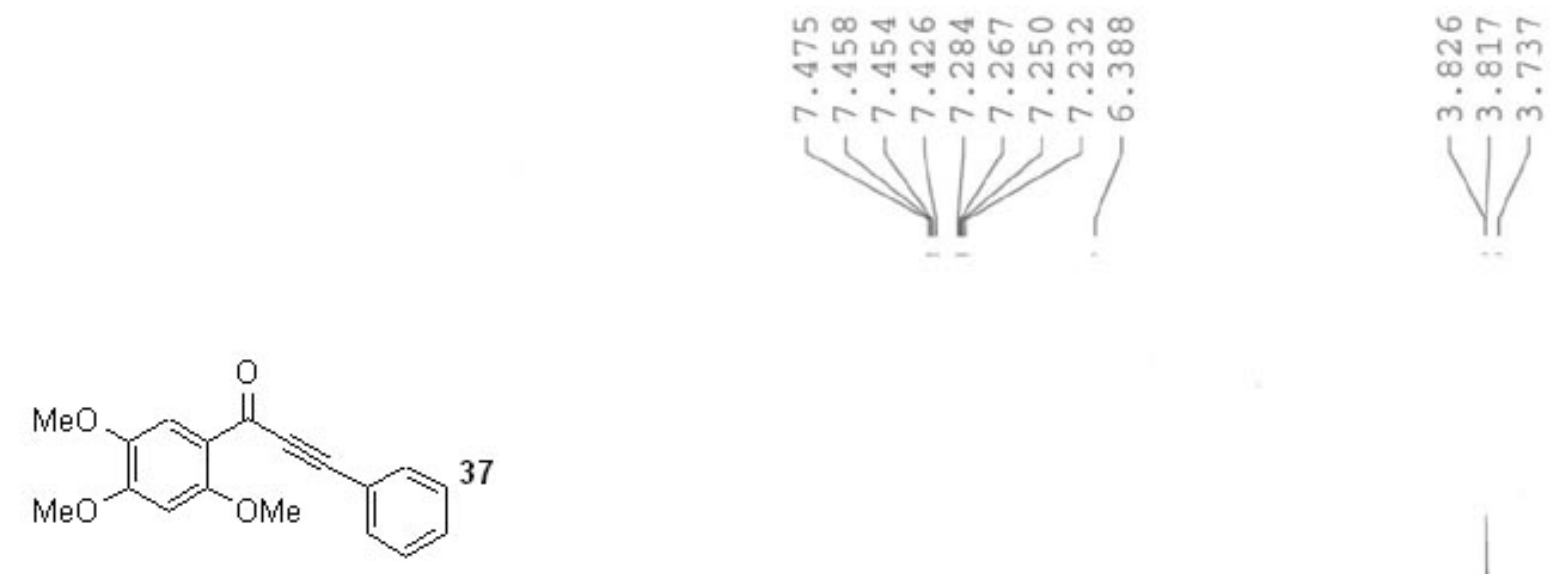

VI

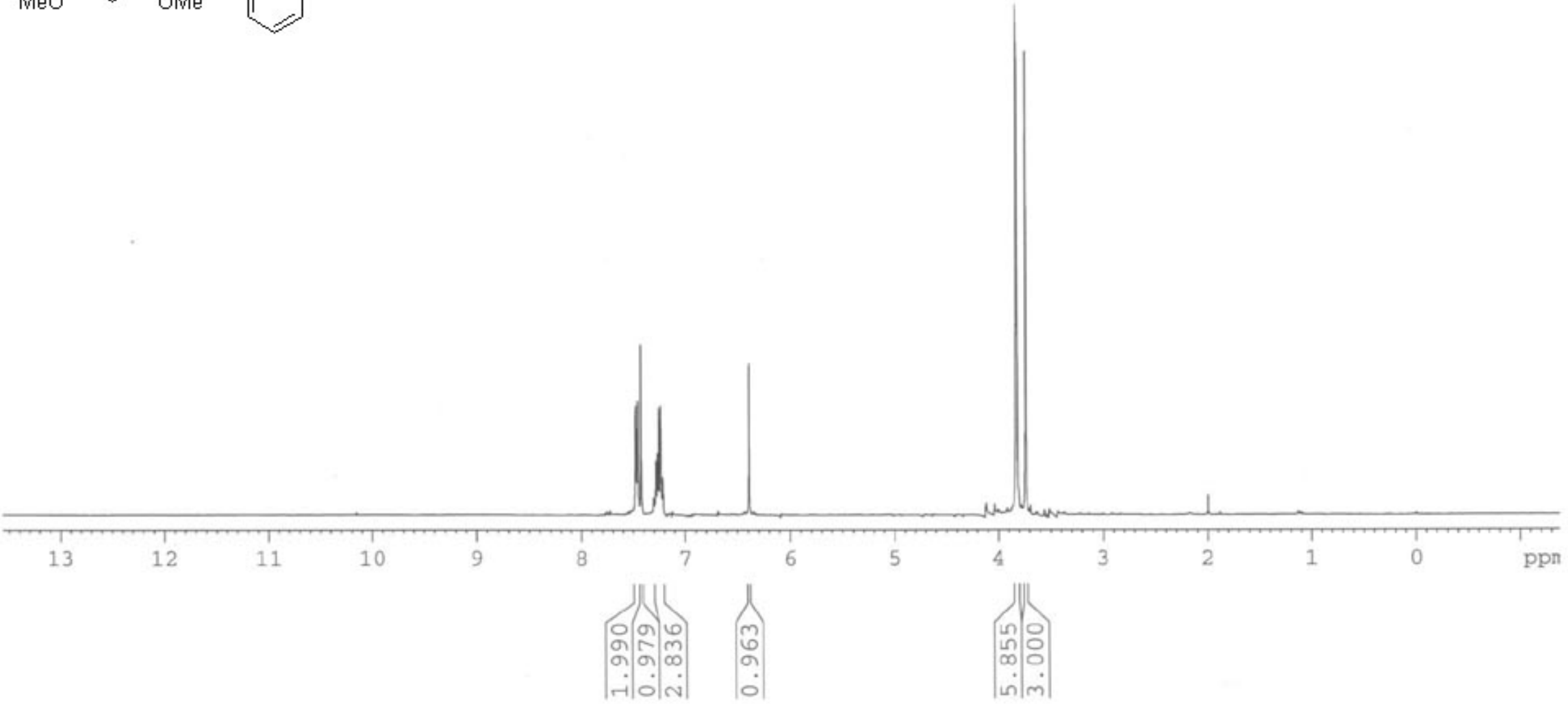



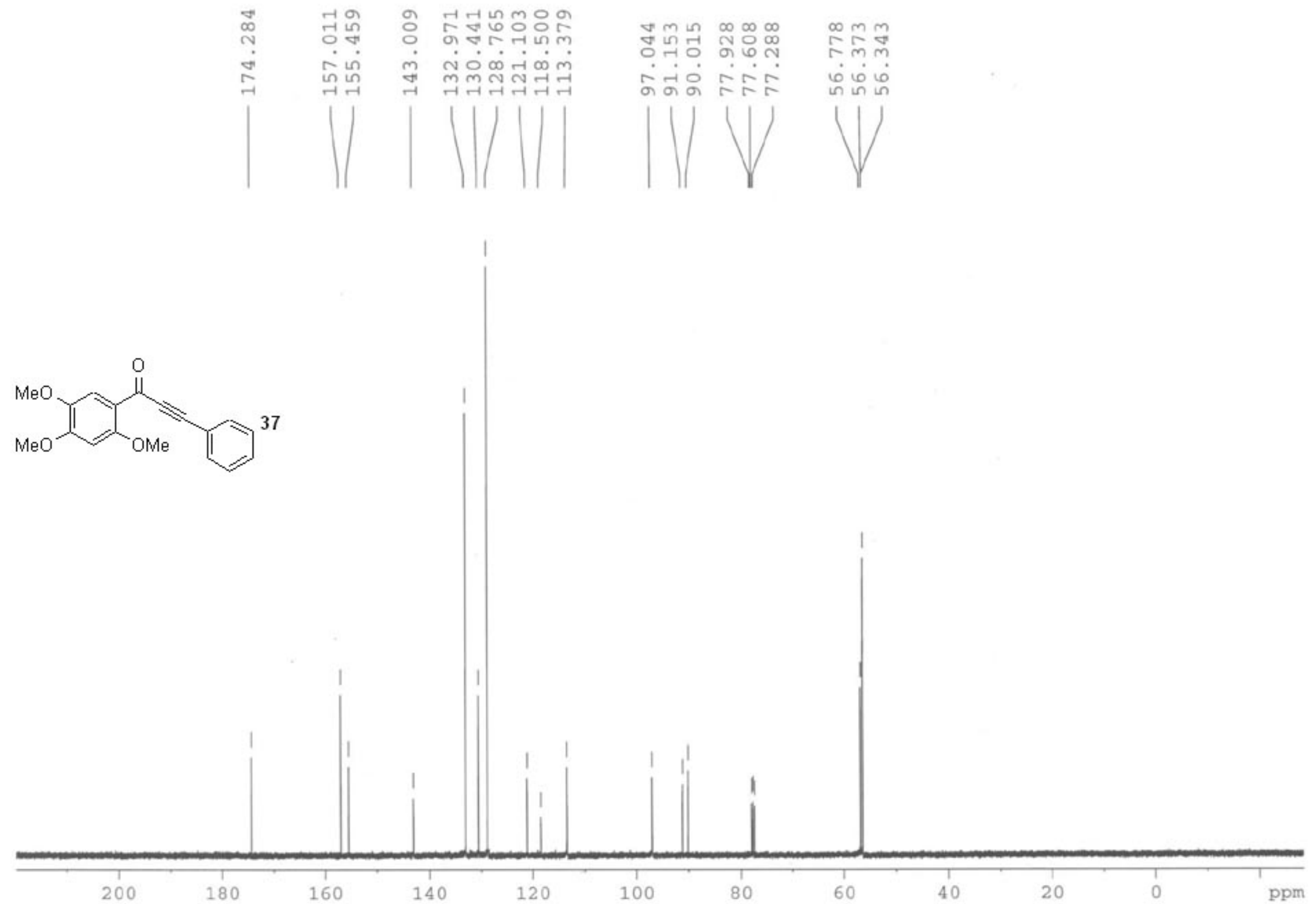


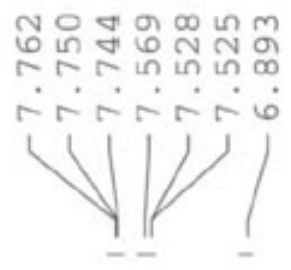

노유

๙ू

m m

V
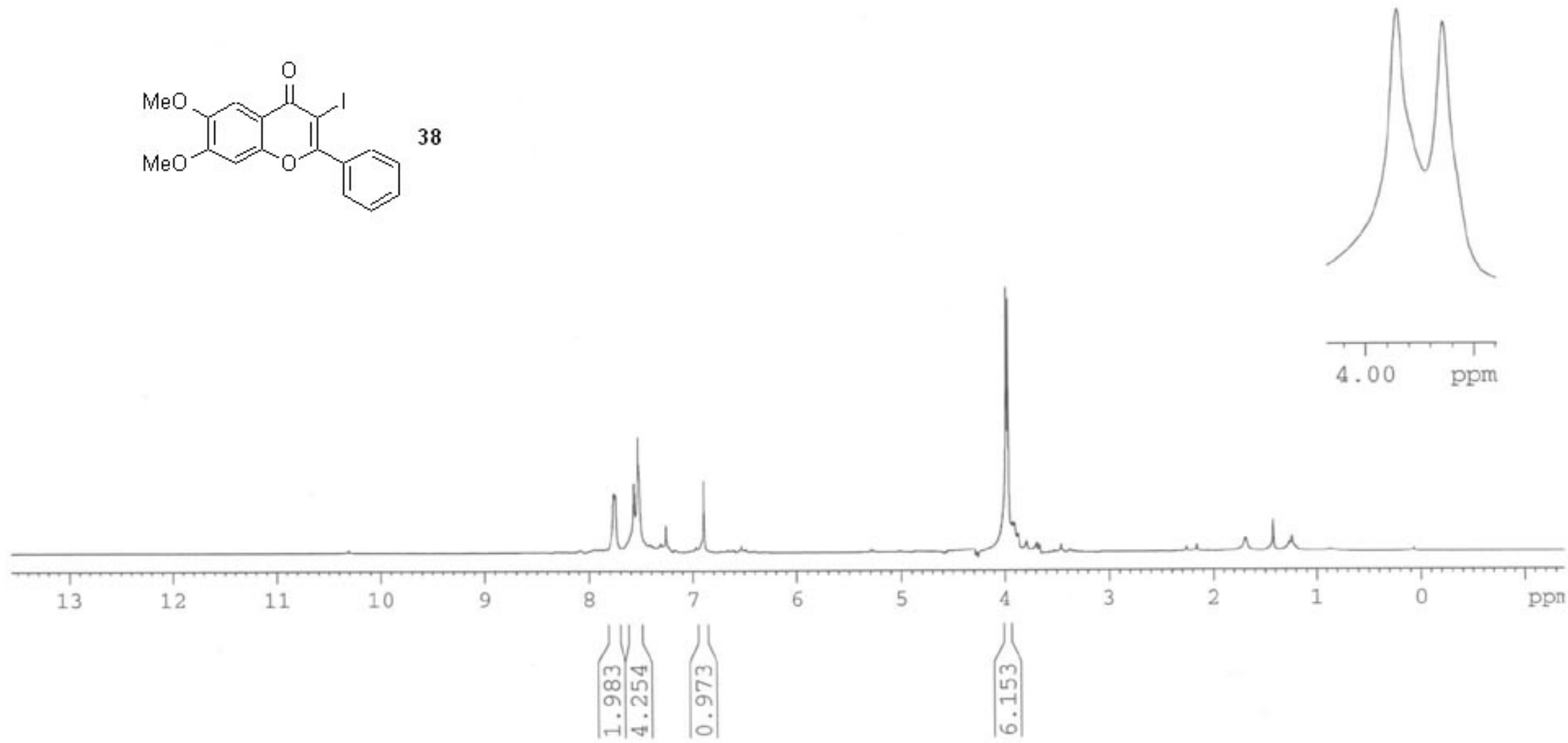

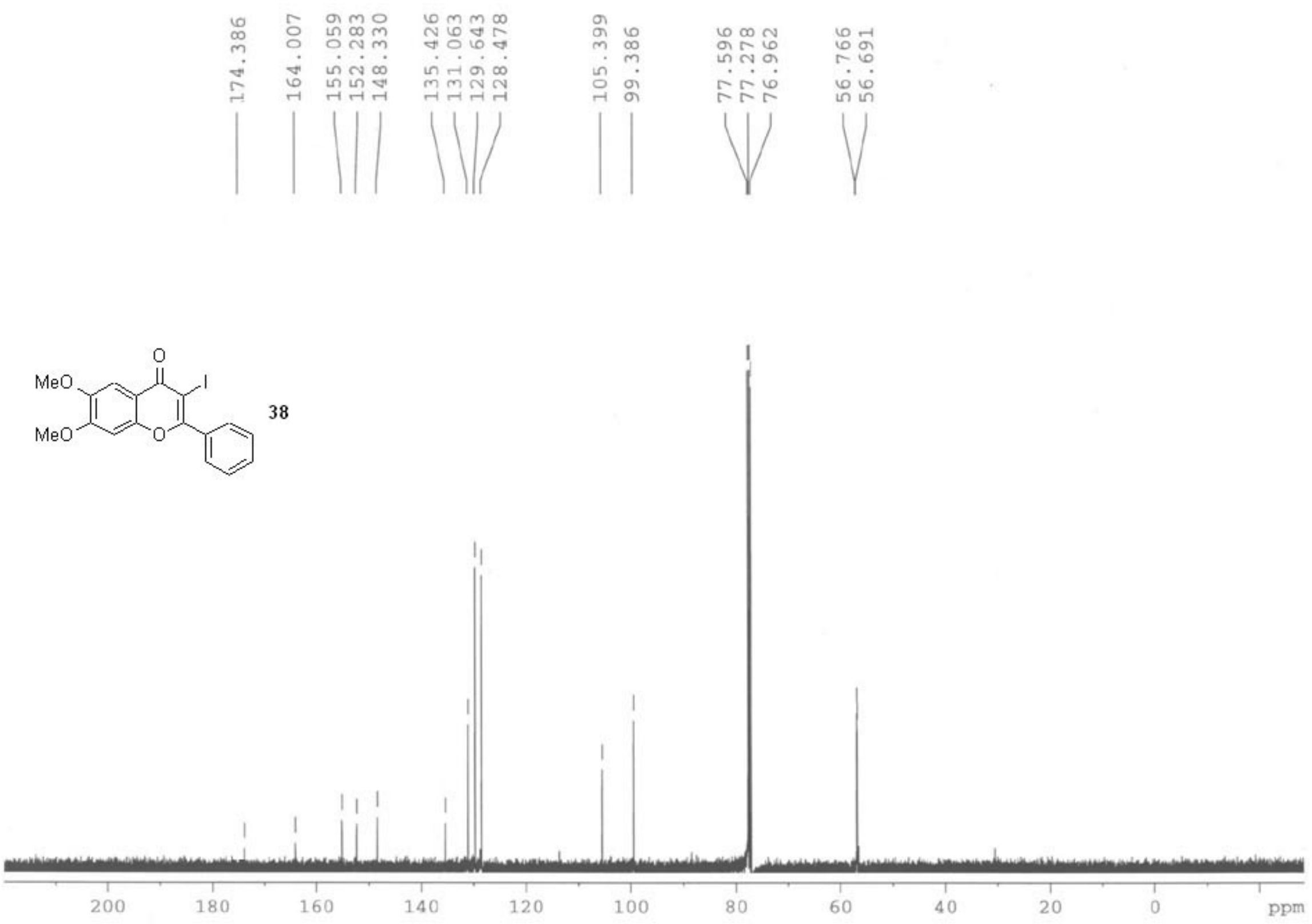

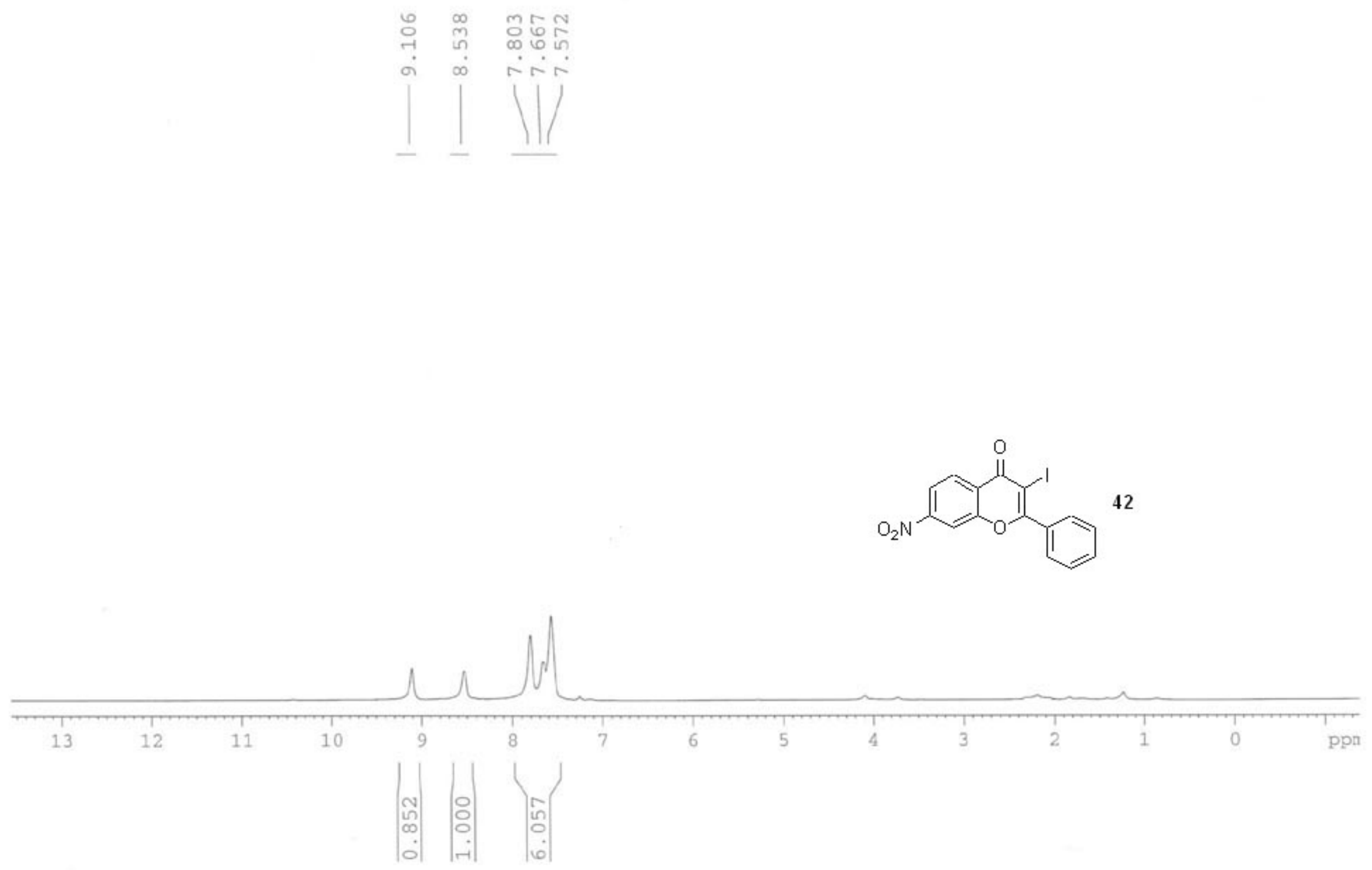


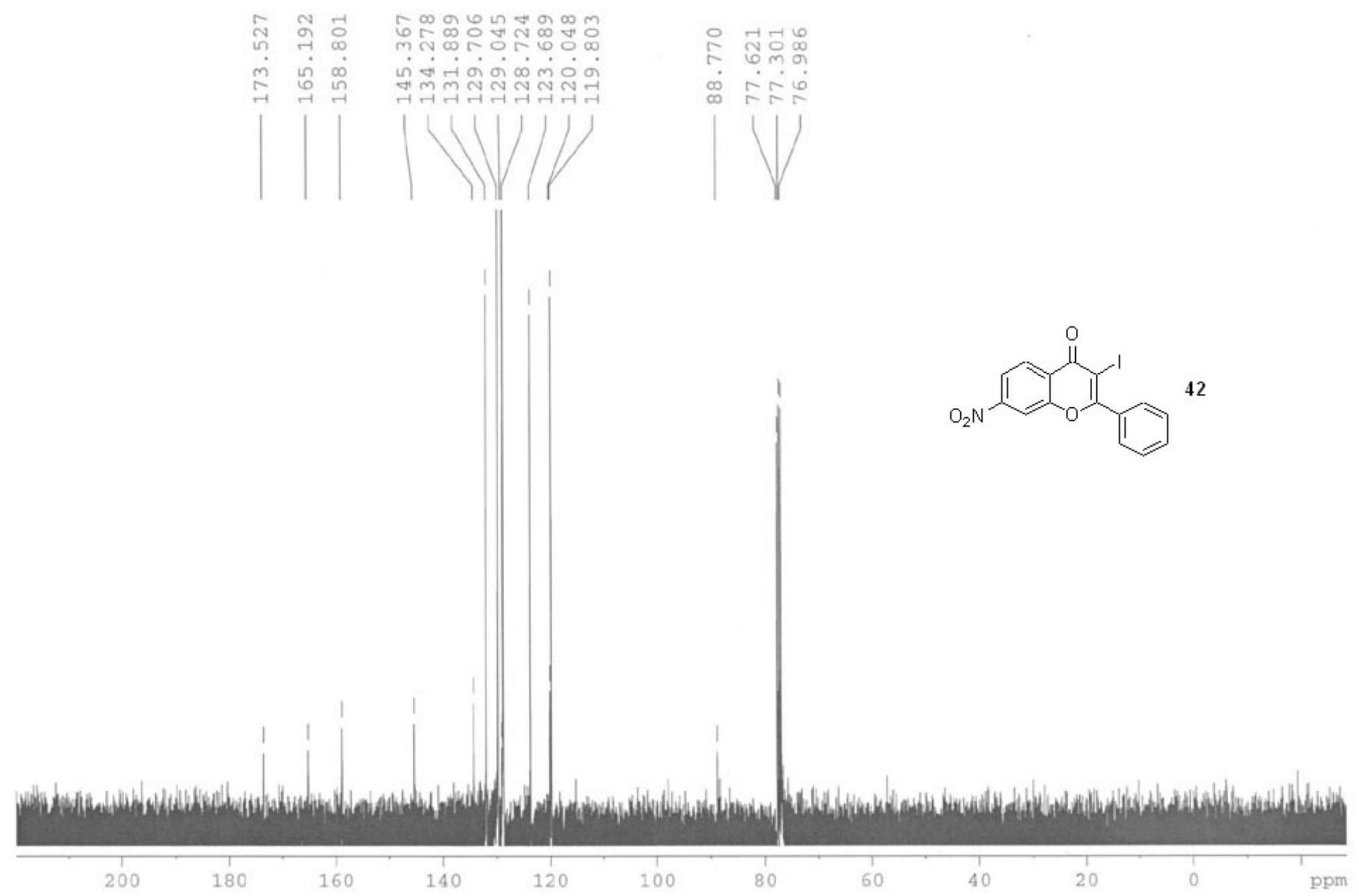



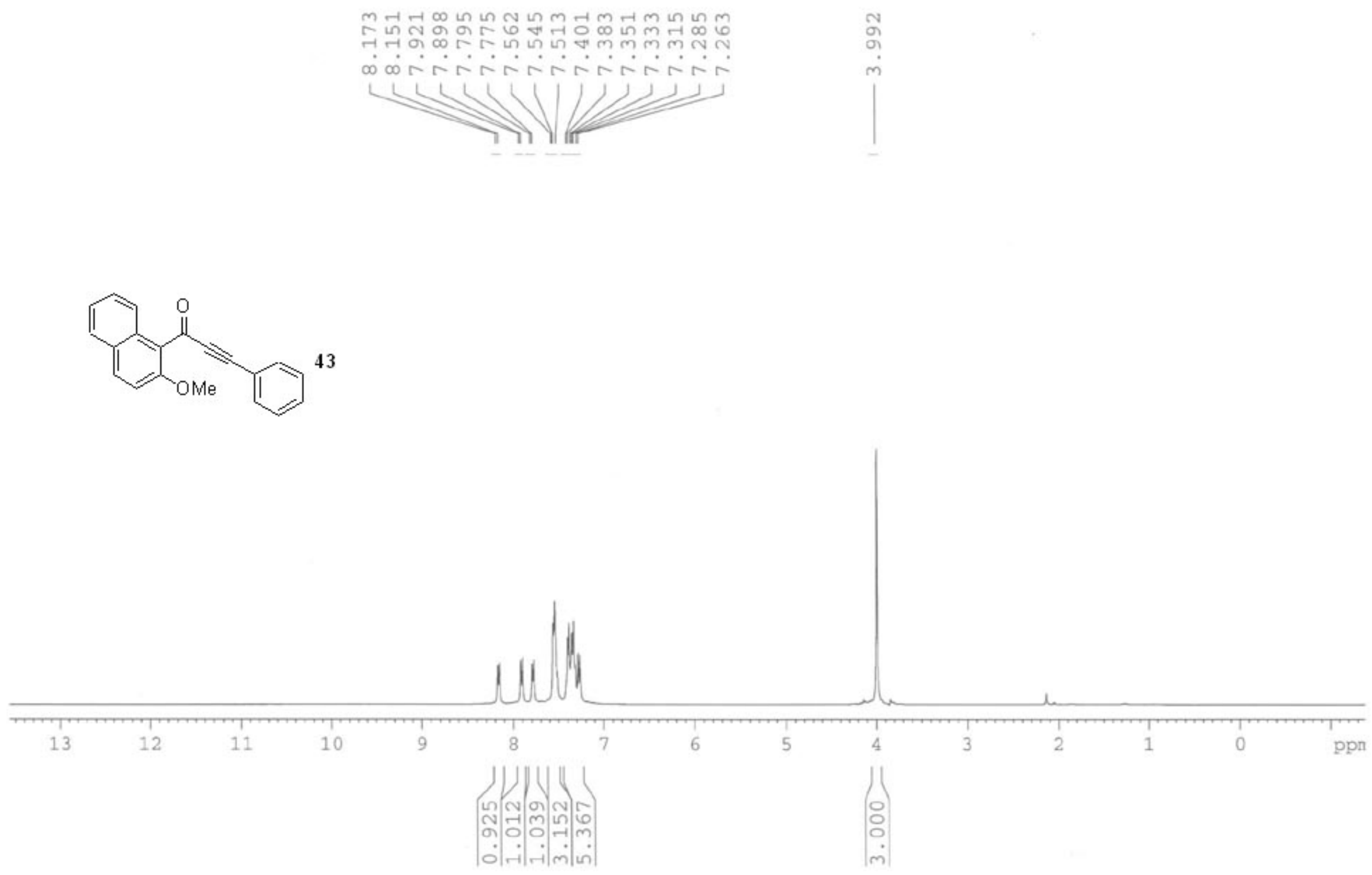

S93 


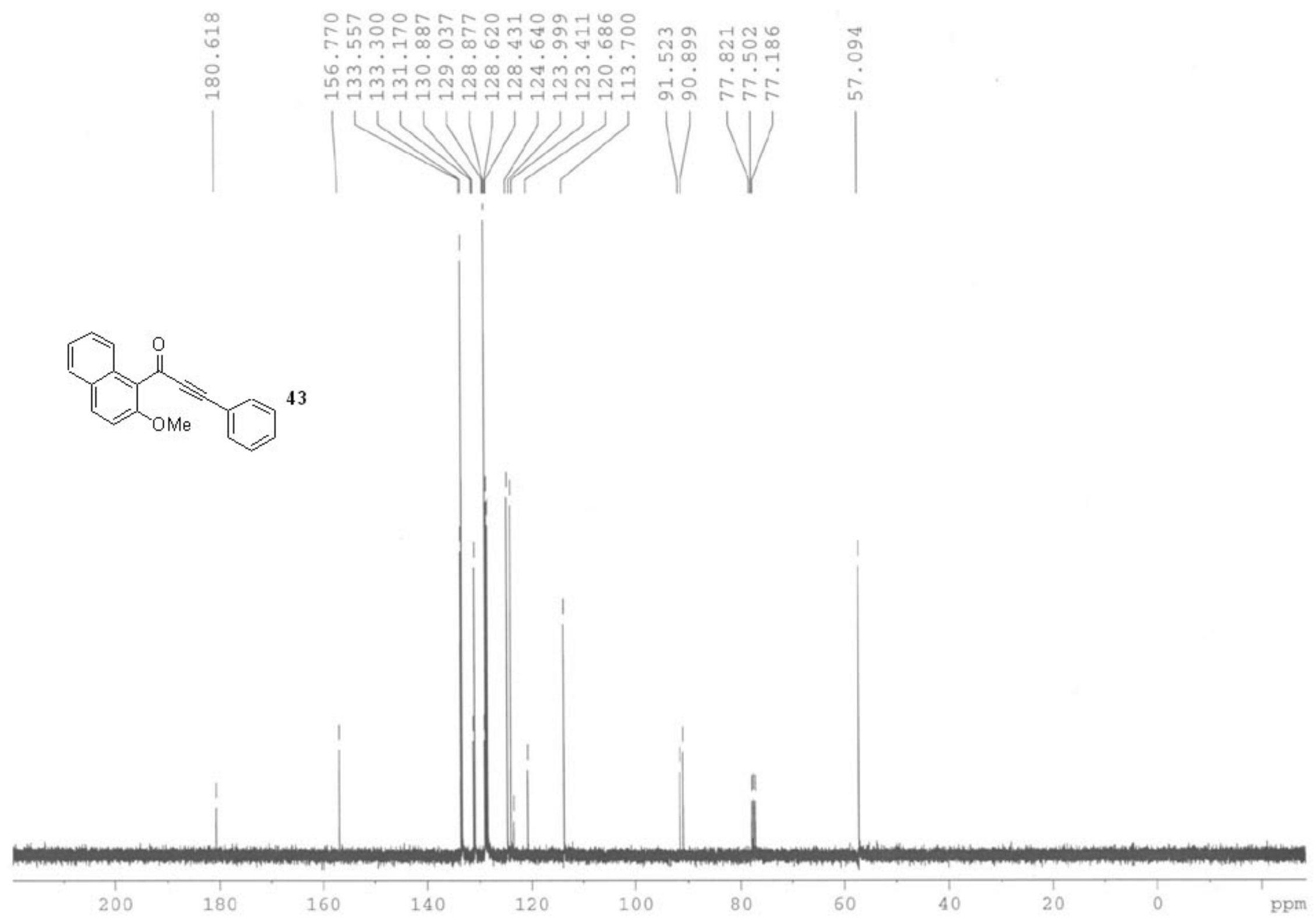




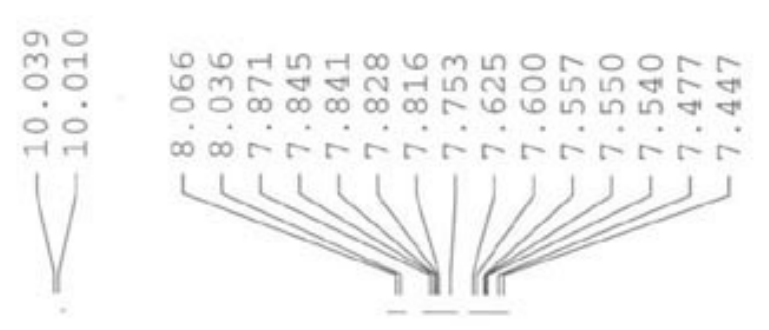<smiles>O=c1c(I)c(-c2ccccc2)oc2ccc3ccccc3c12</smiles>

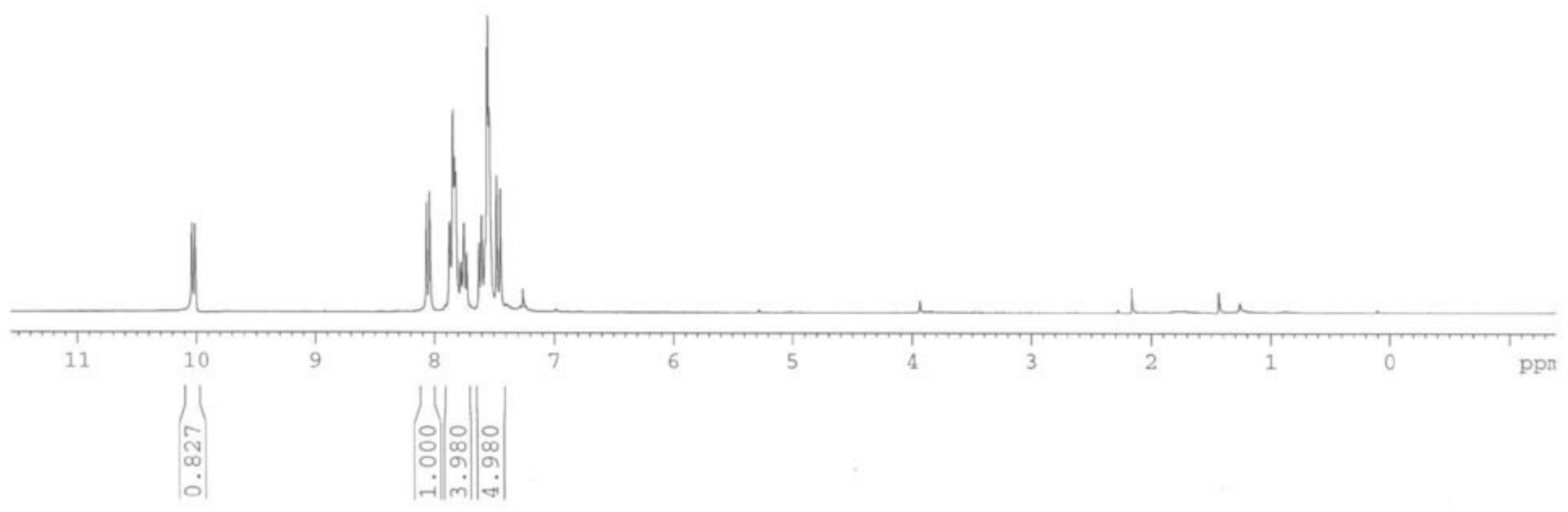

S95 


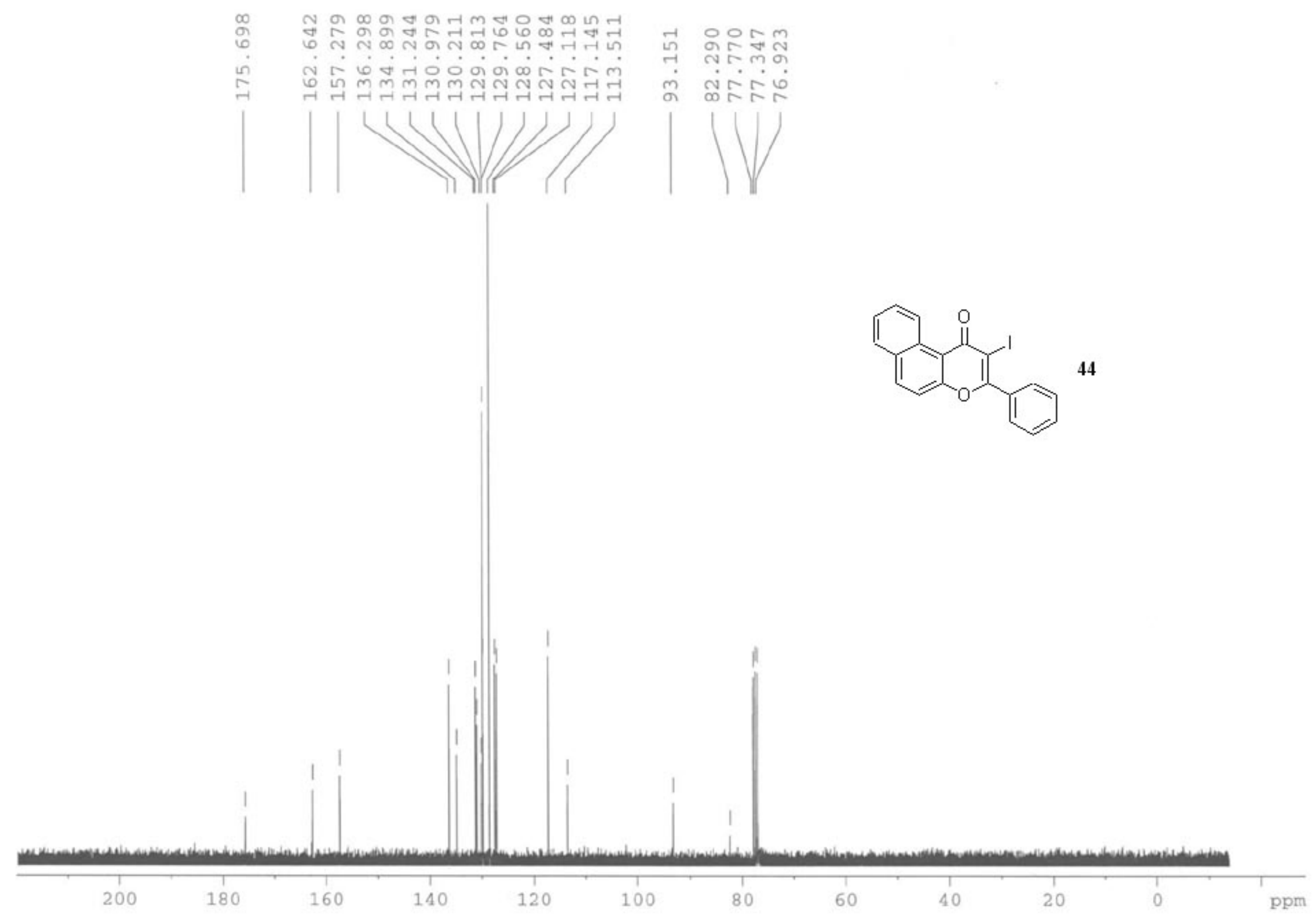



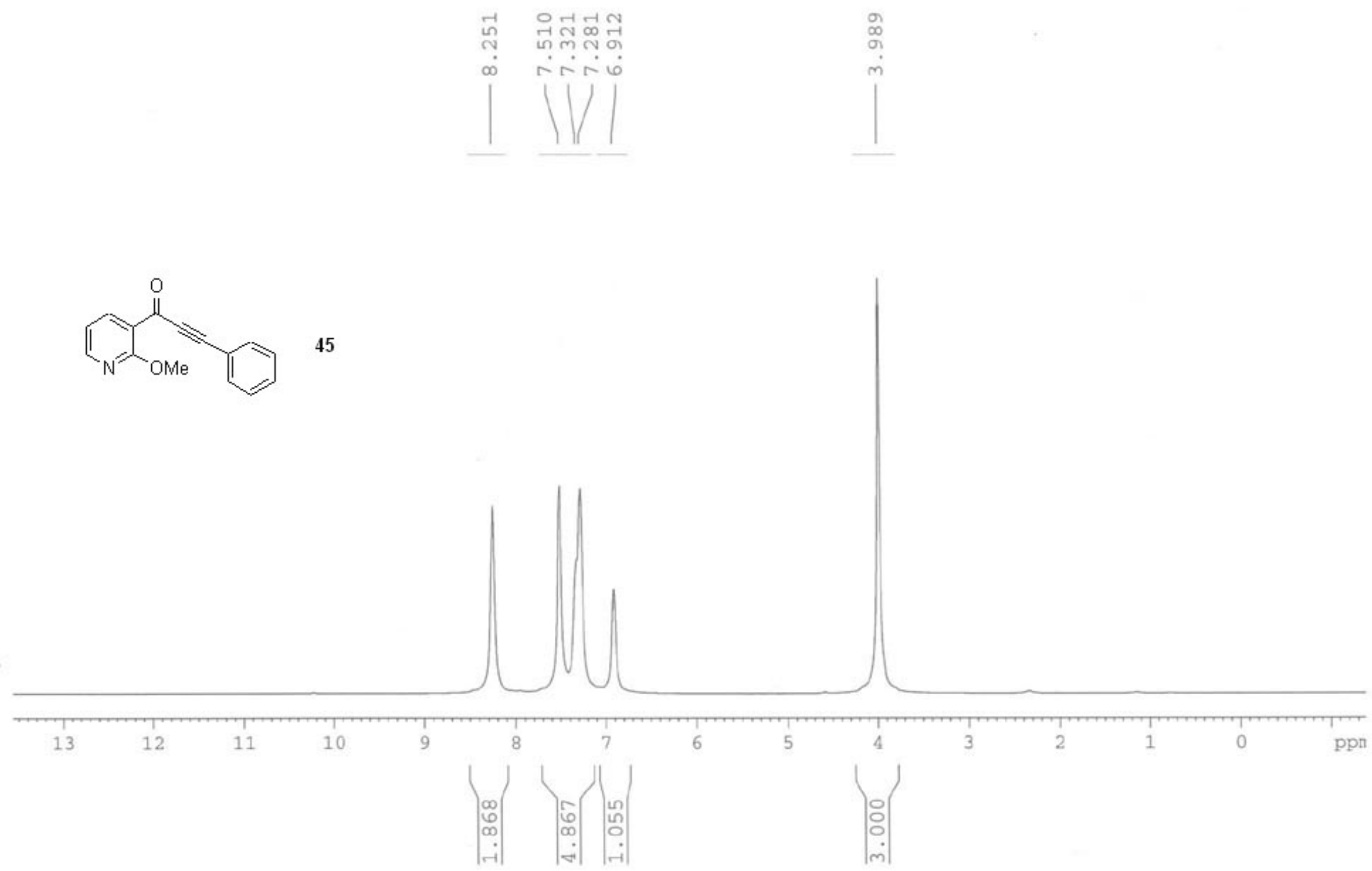


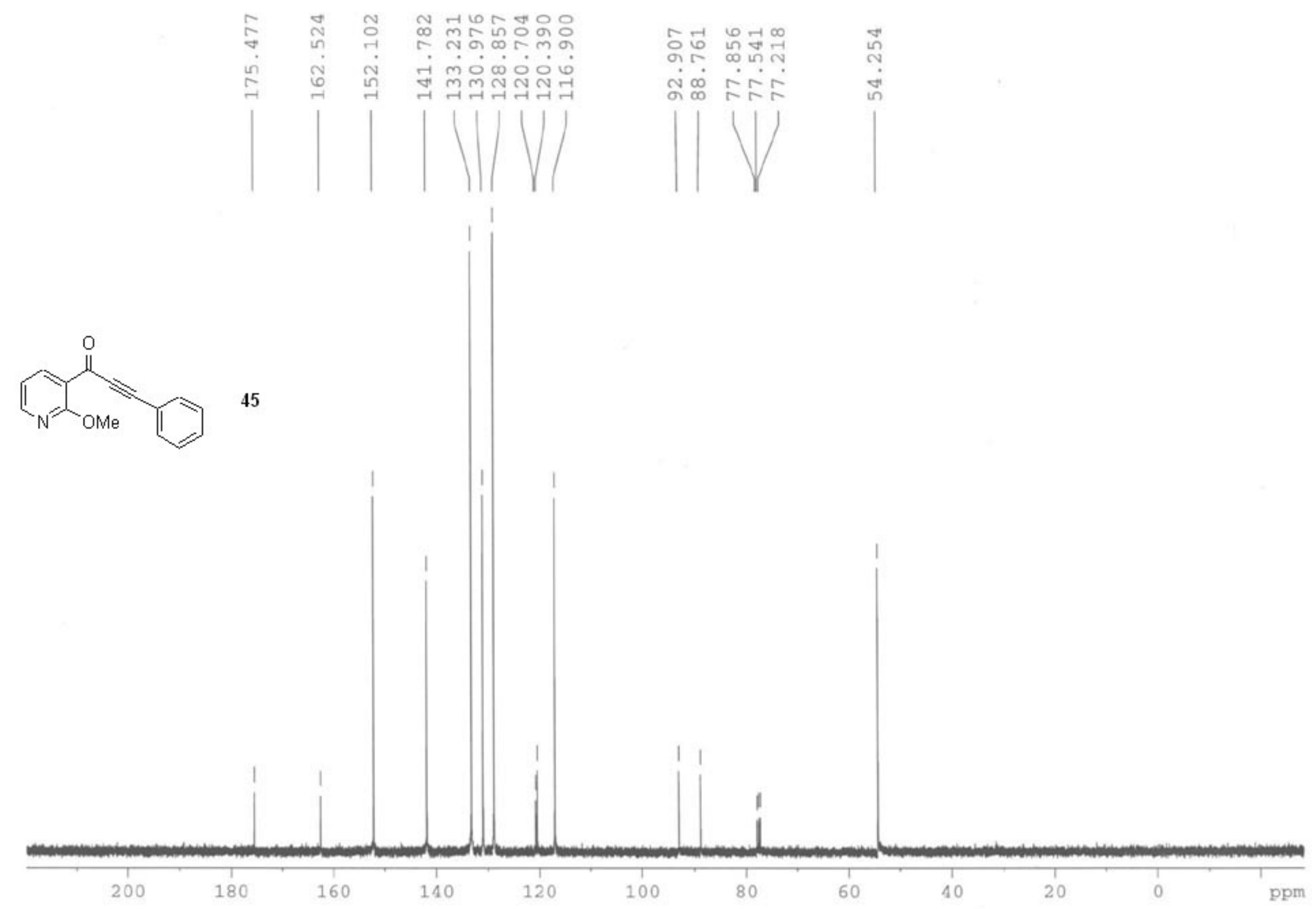


$60-\infty \pi$ แ $9 \pi m \forall n$

N

$\dot{\infty} \dot{\infty} \dot{\infty} \dot{\infty} \dot{\sim} \dot{n} \dot{r}$

S. 1112

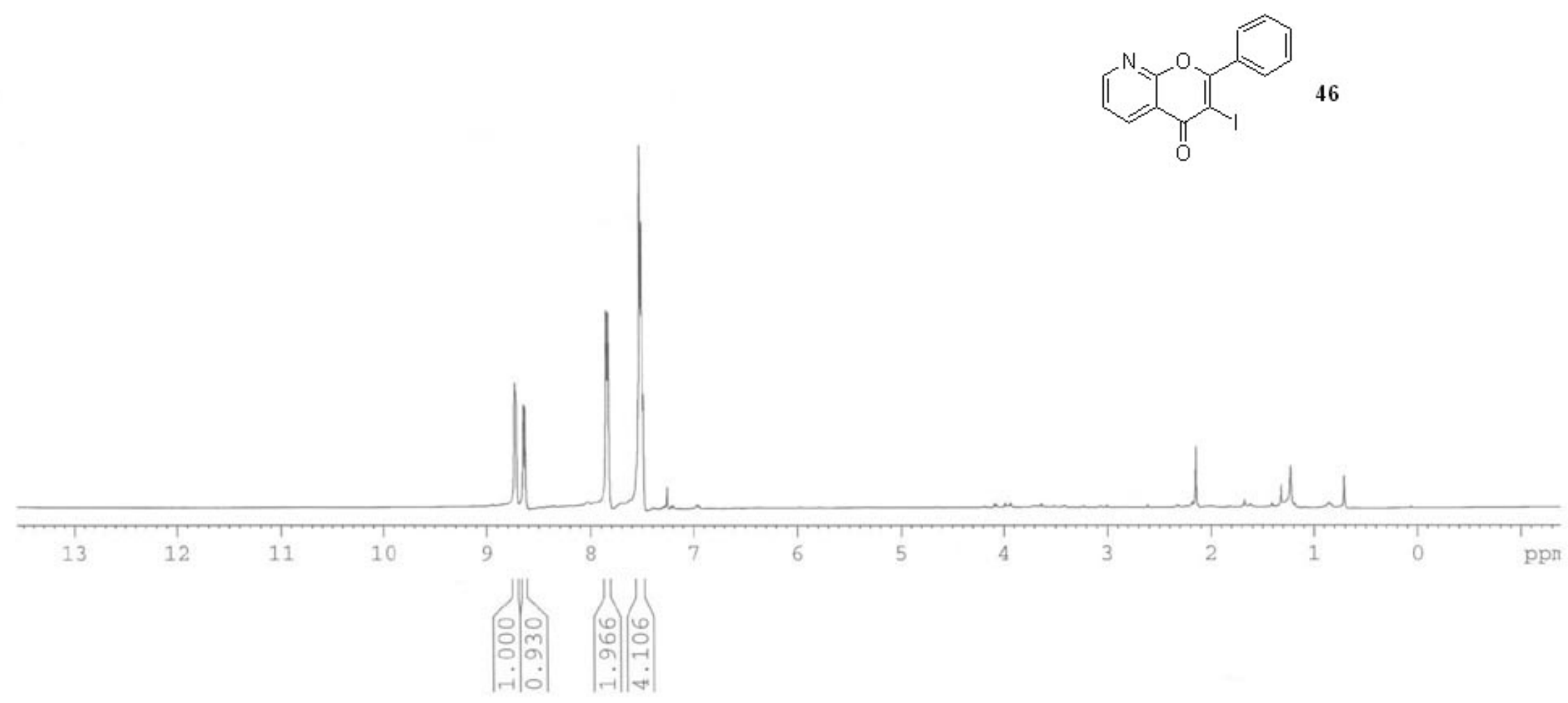




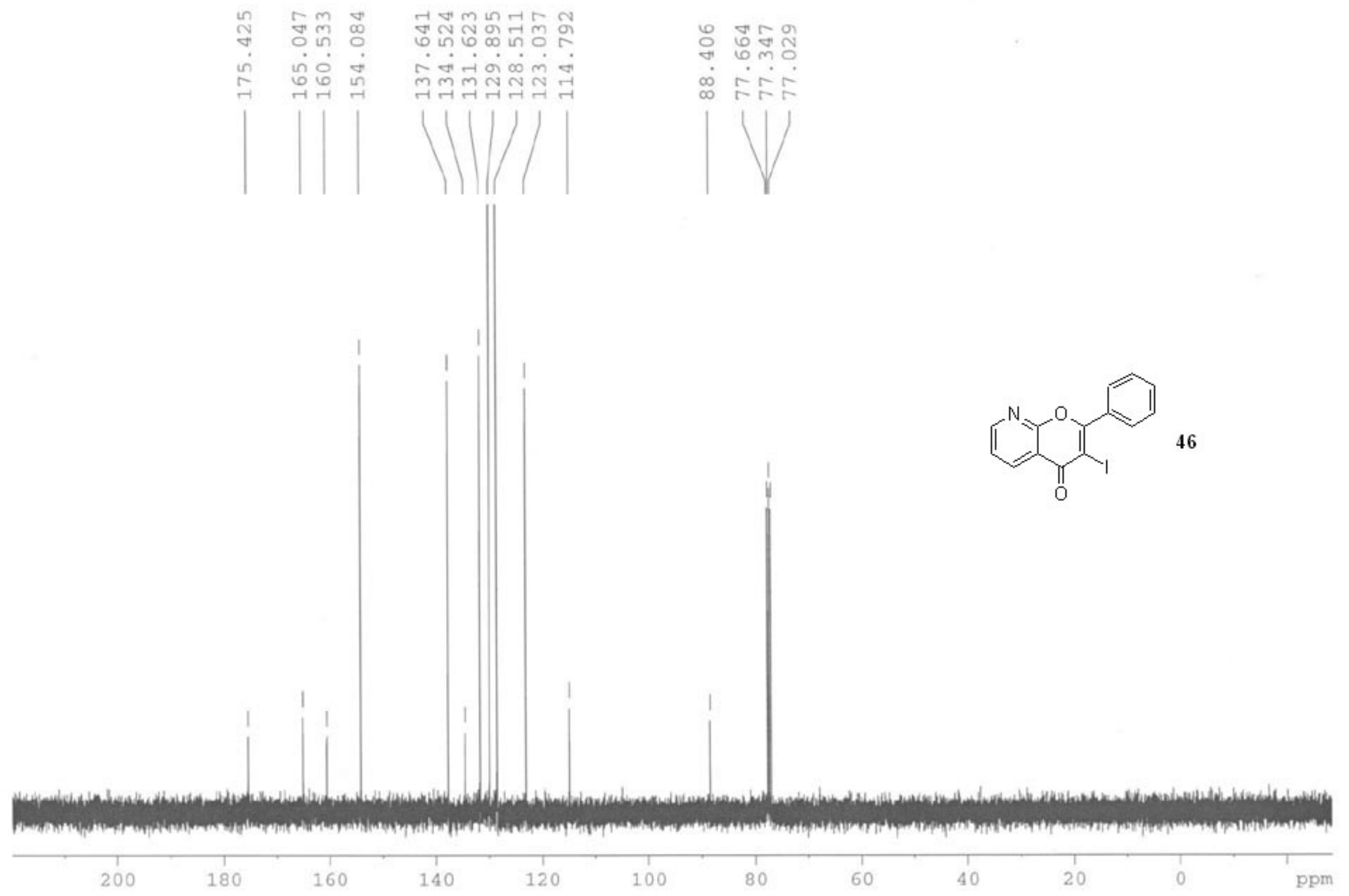




\section{8-30-SMe-cyclohexene-ketone}

m N N

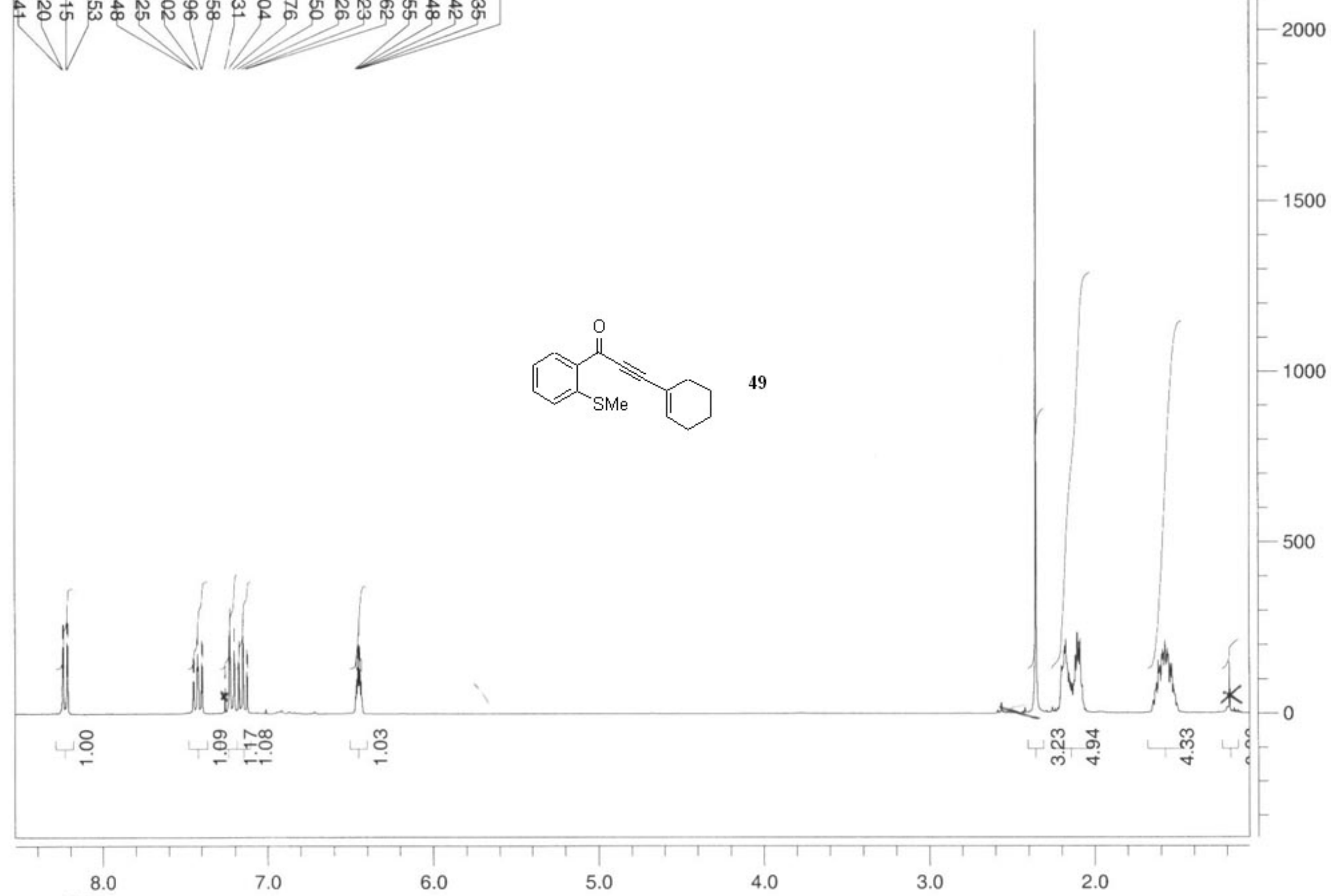

ppm (f1) 


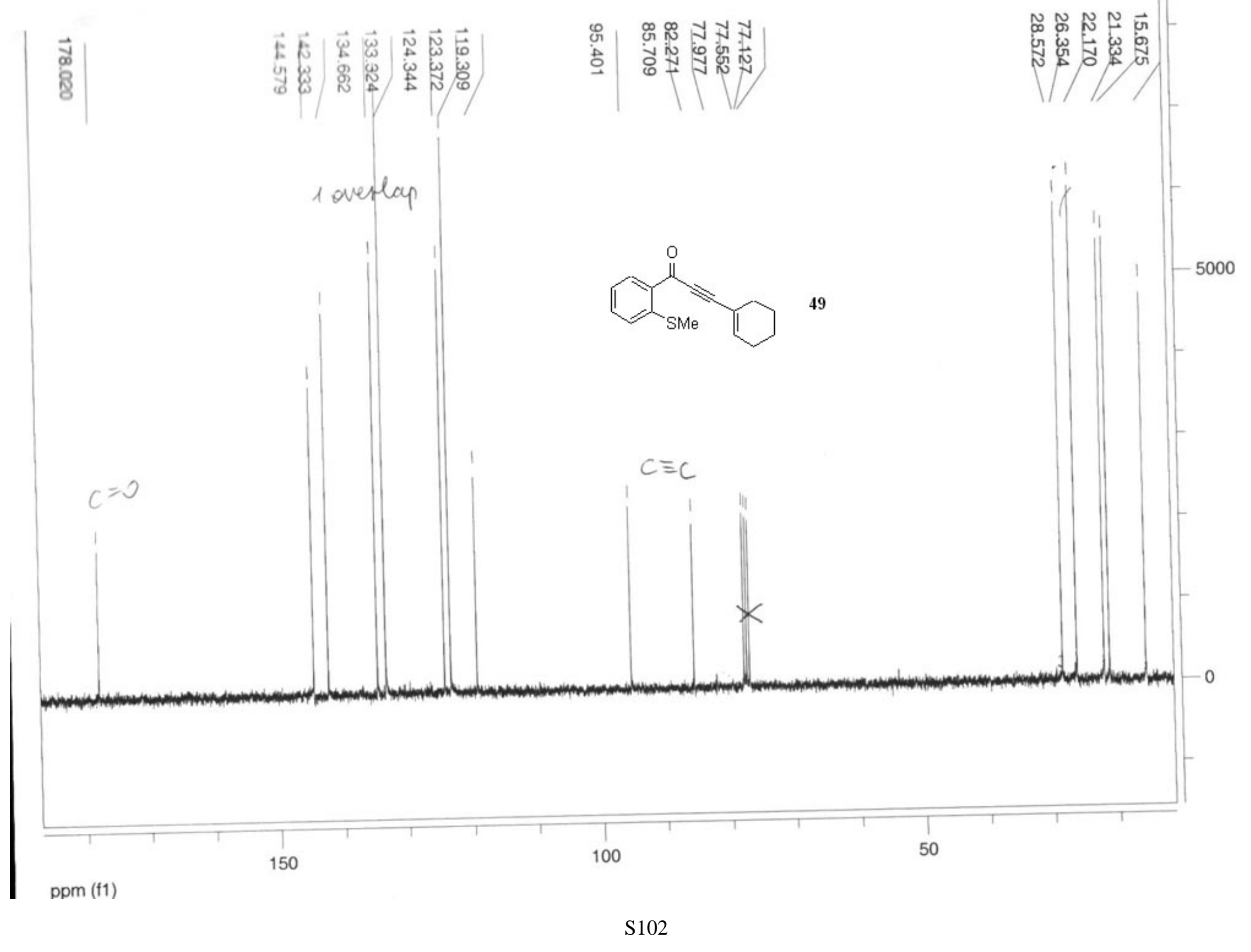


스요

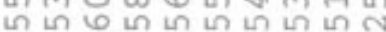

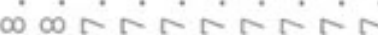

$\checkmark$

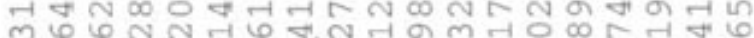
m

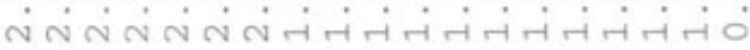

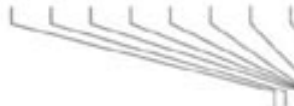

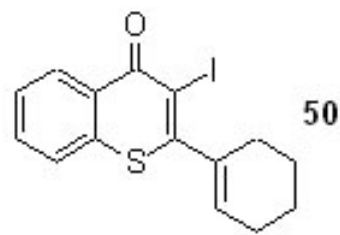

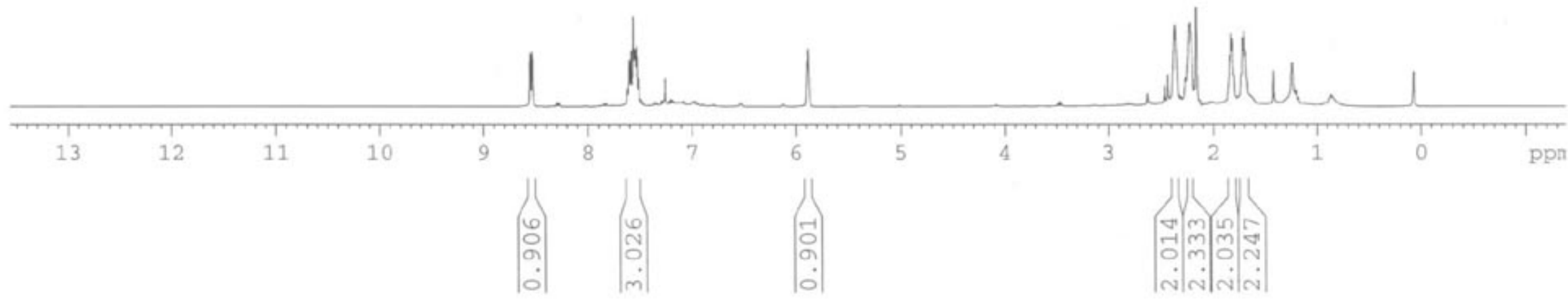




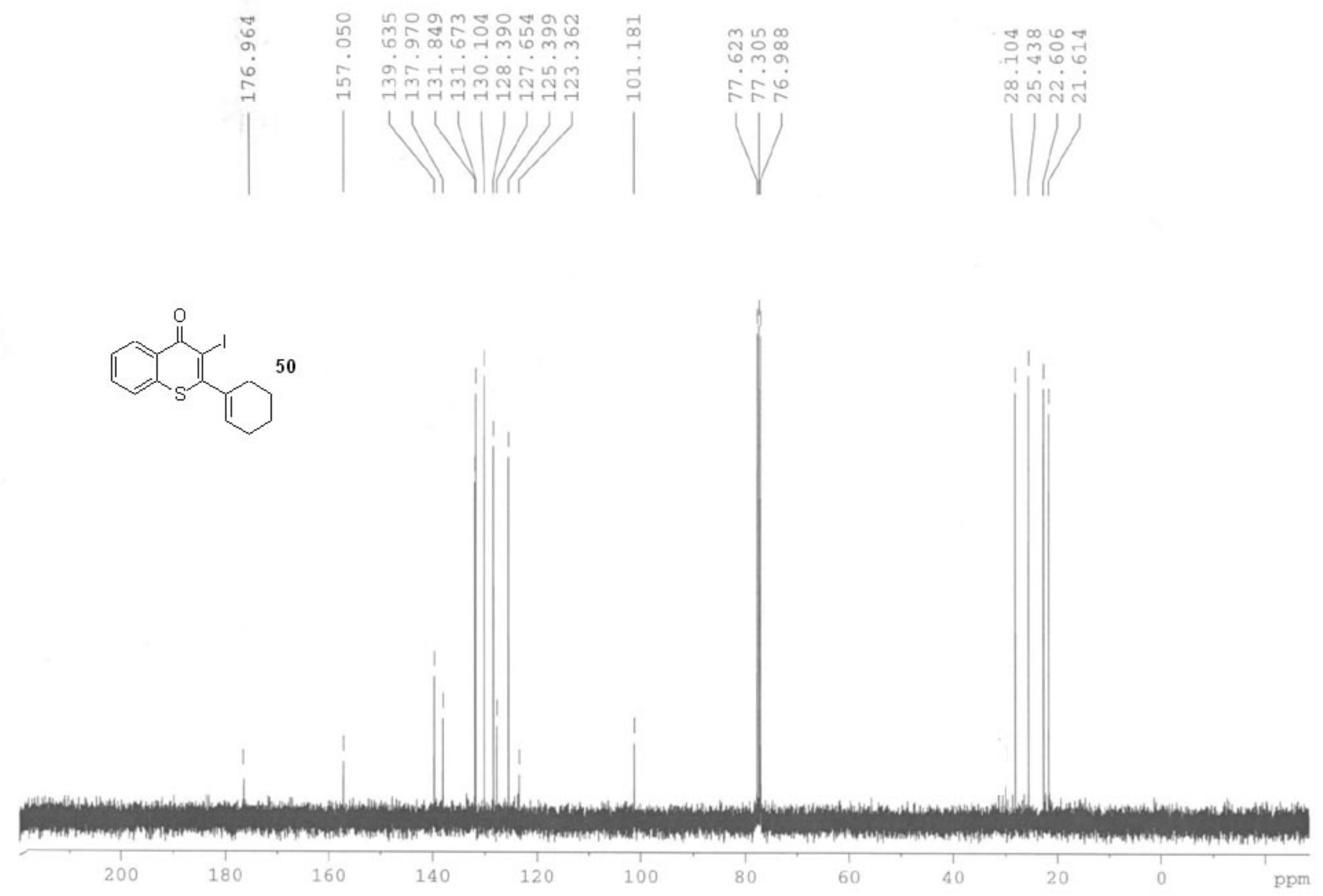




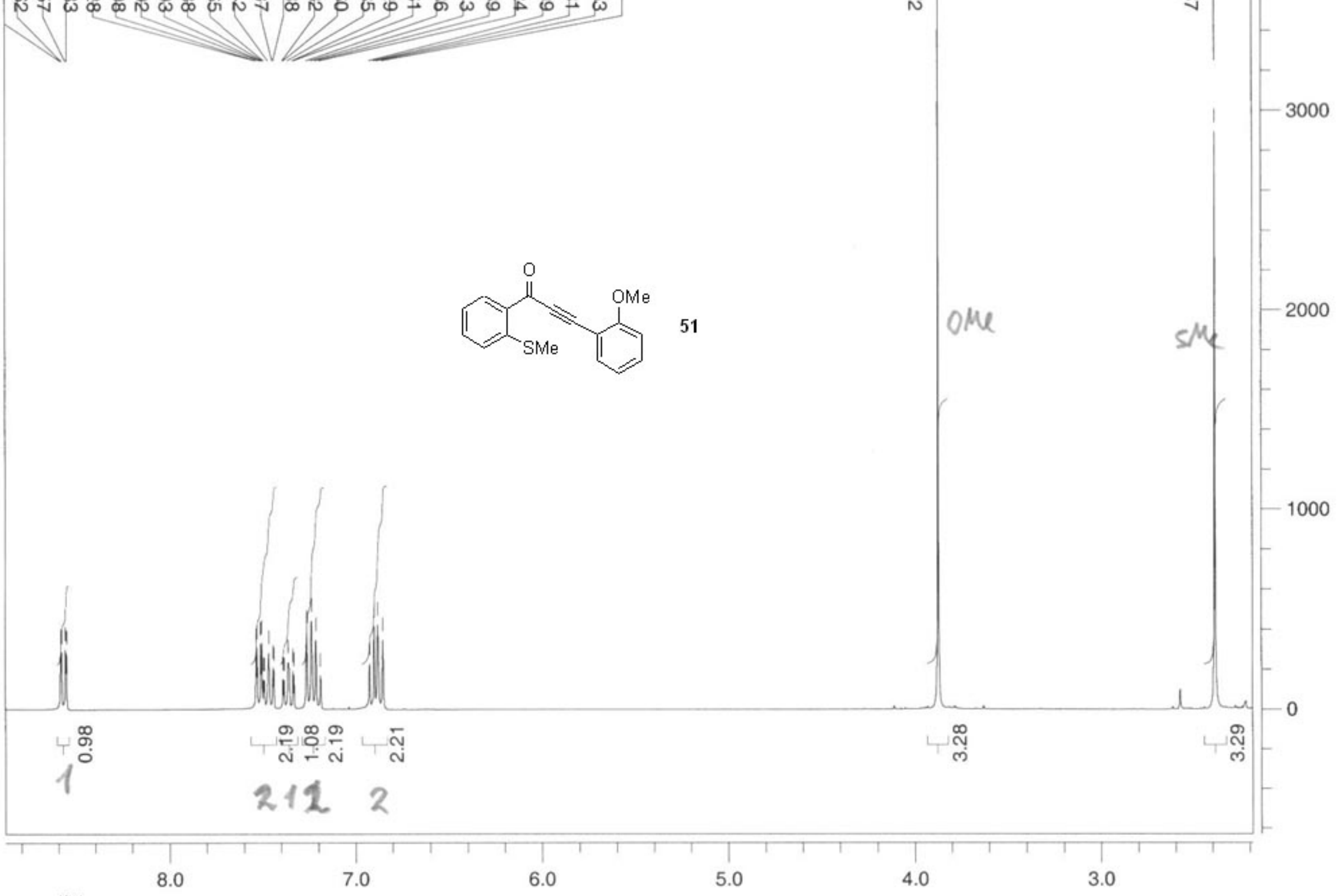

ppm (f1) 


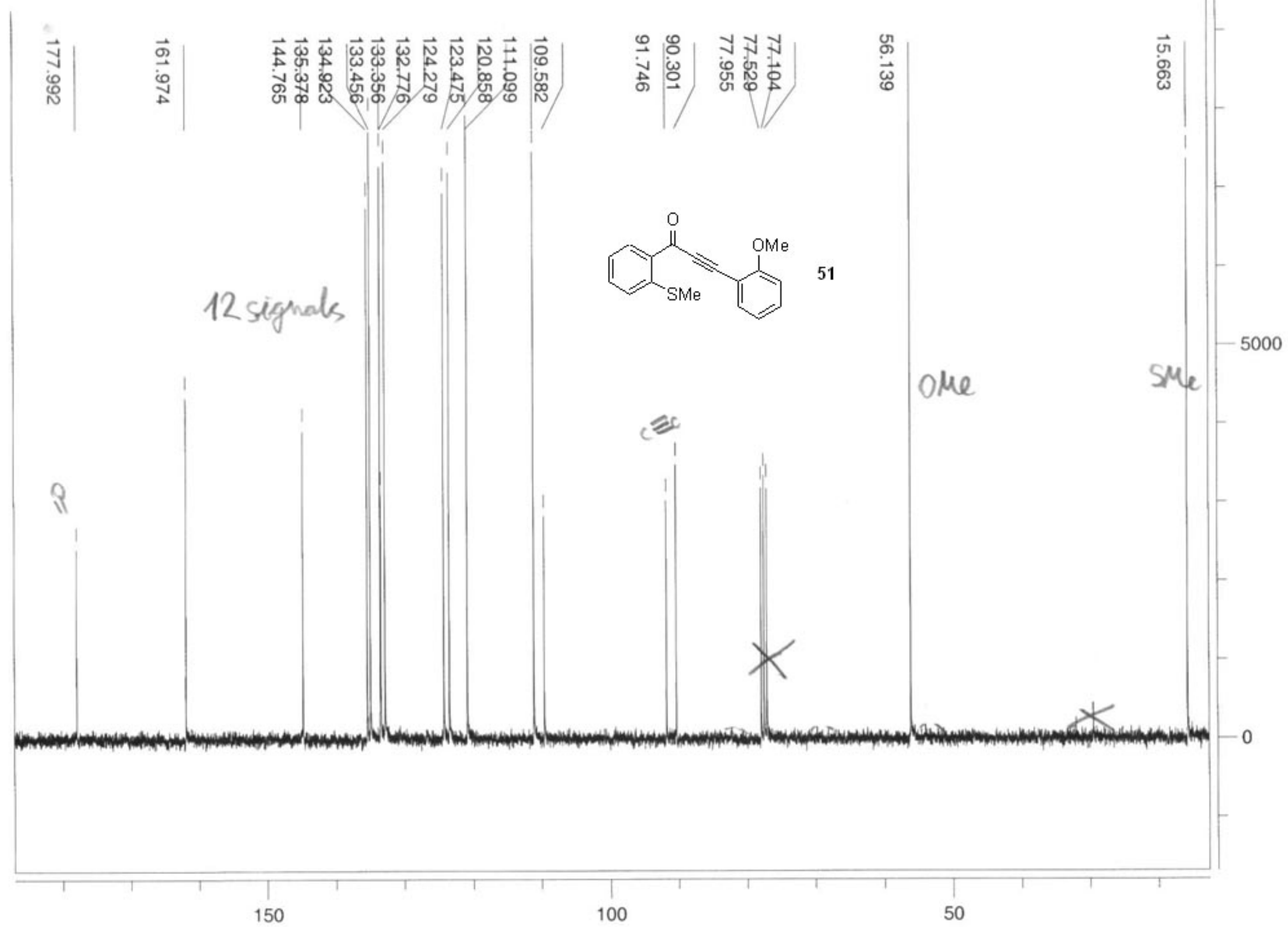

ppm (f1) 


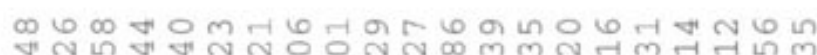
ที่ ำน

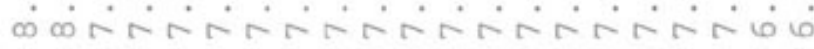

$\stackrel{6}{6}$

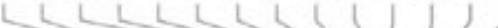
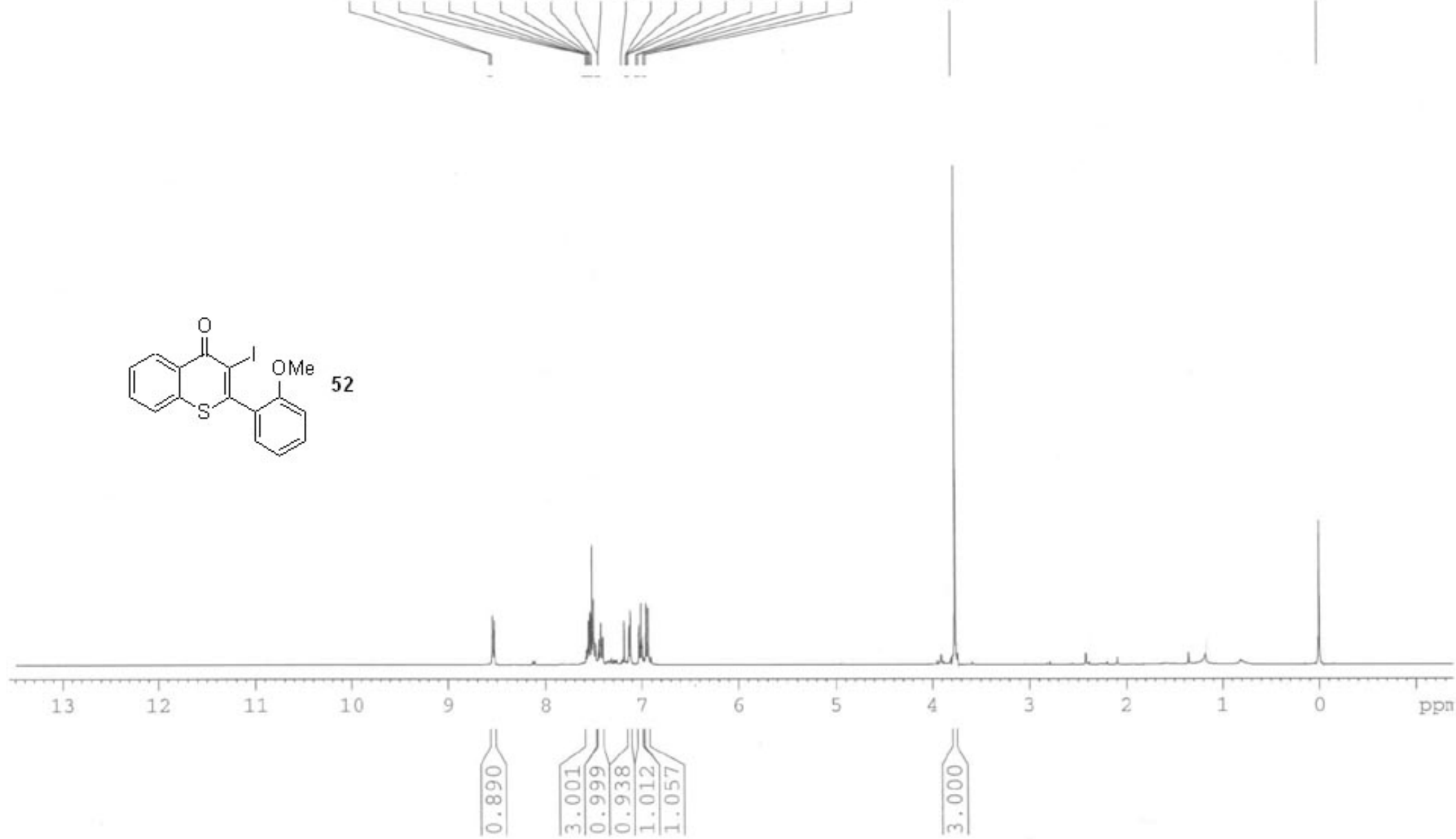

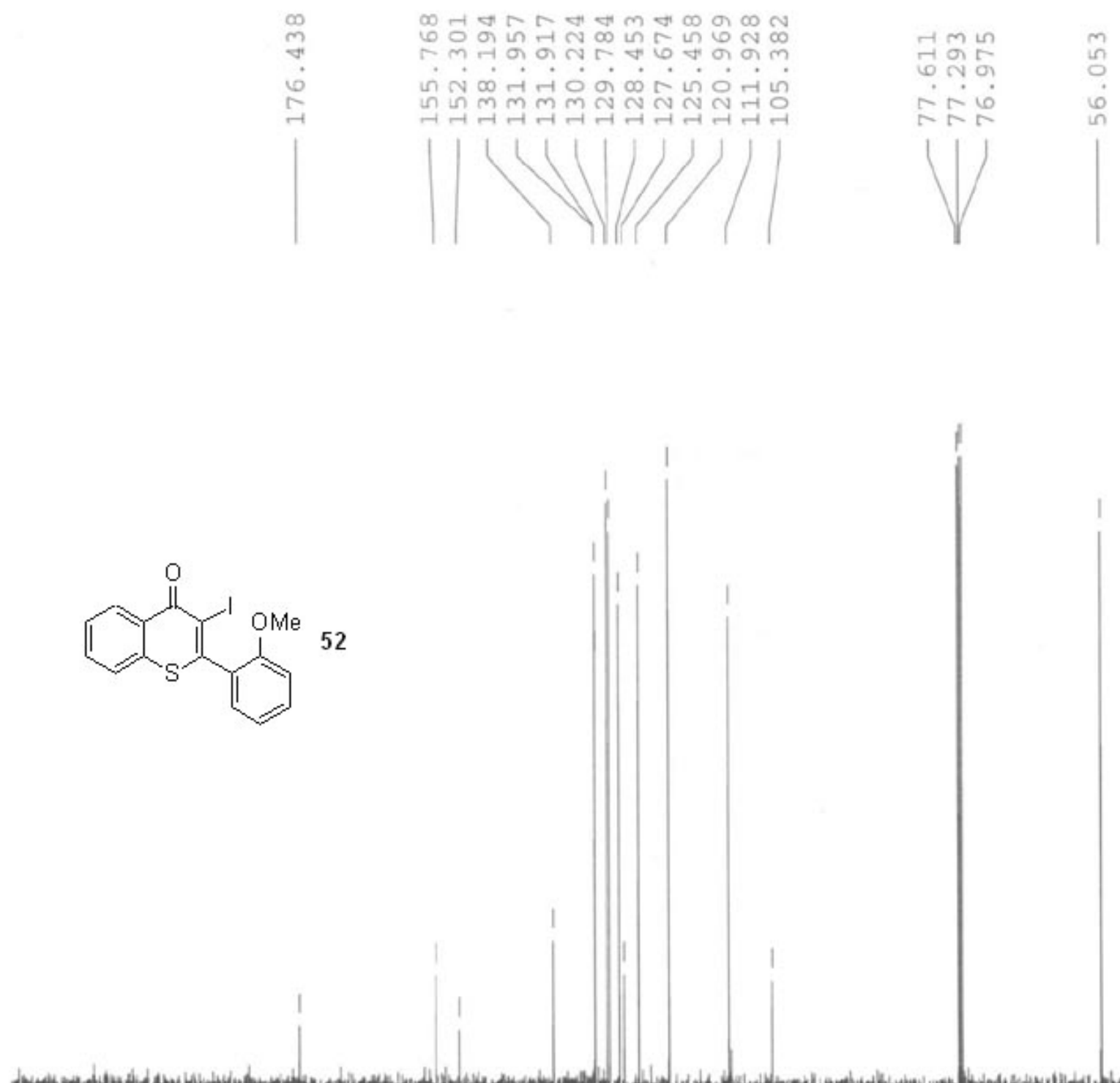

200

180

160

140

120

100

80

60

40

20

0

ppm 


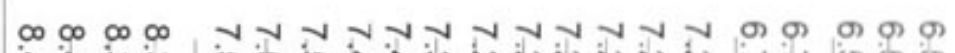

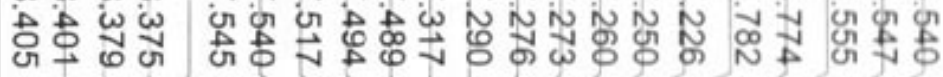

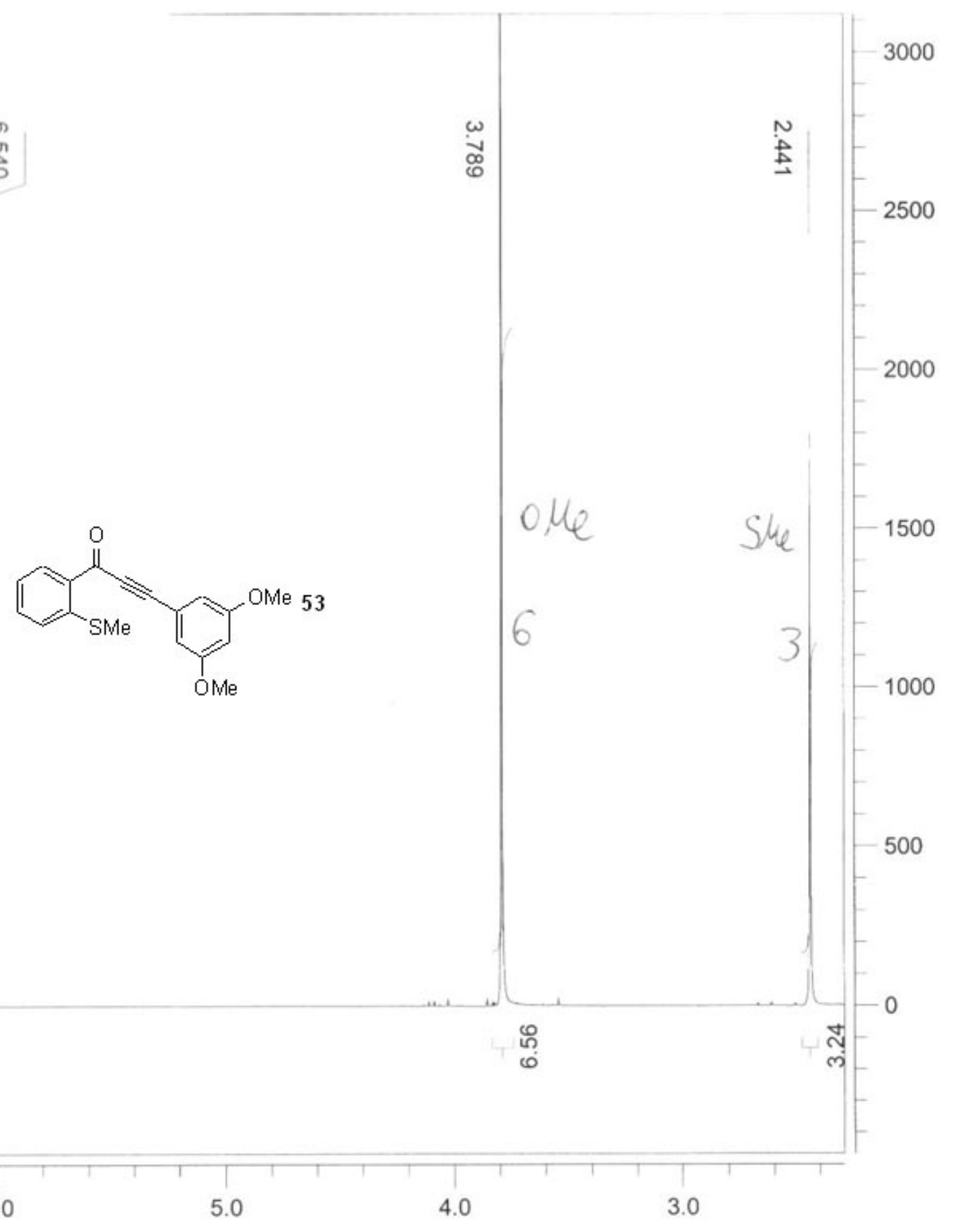

ppm (f1) 


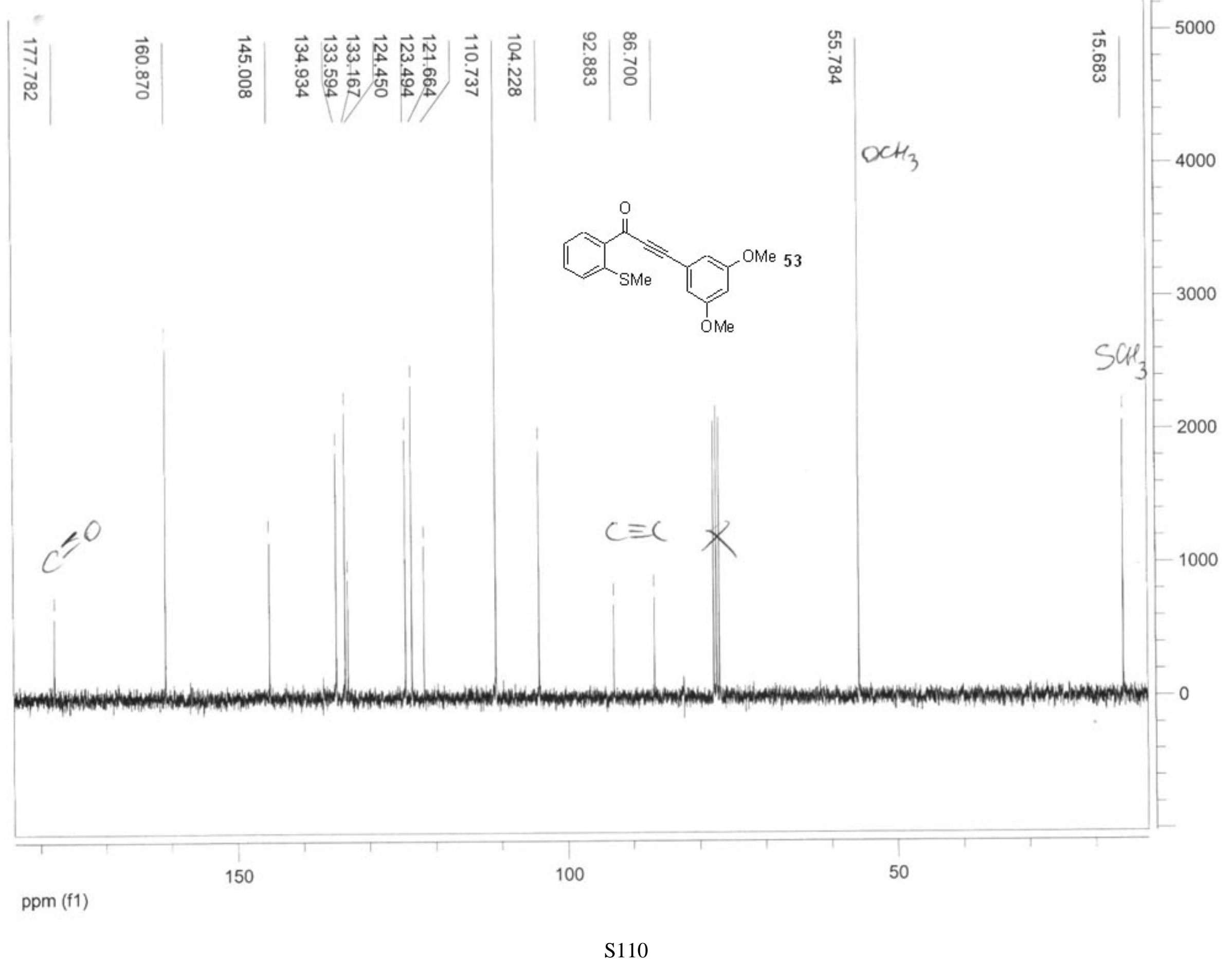




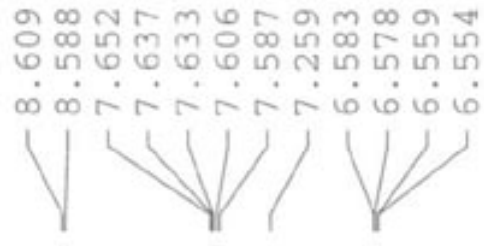

꾹ㄱ

$\infty$

ले
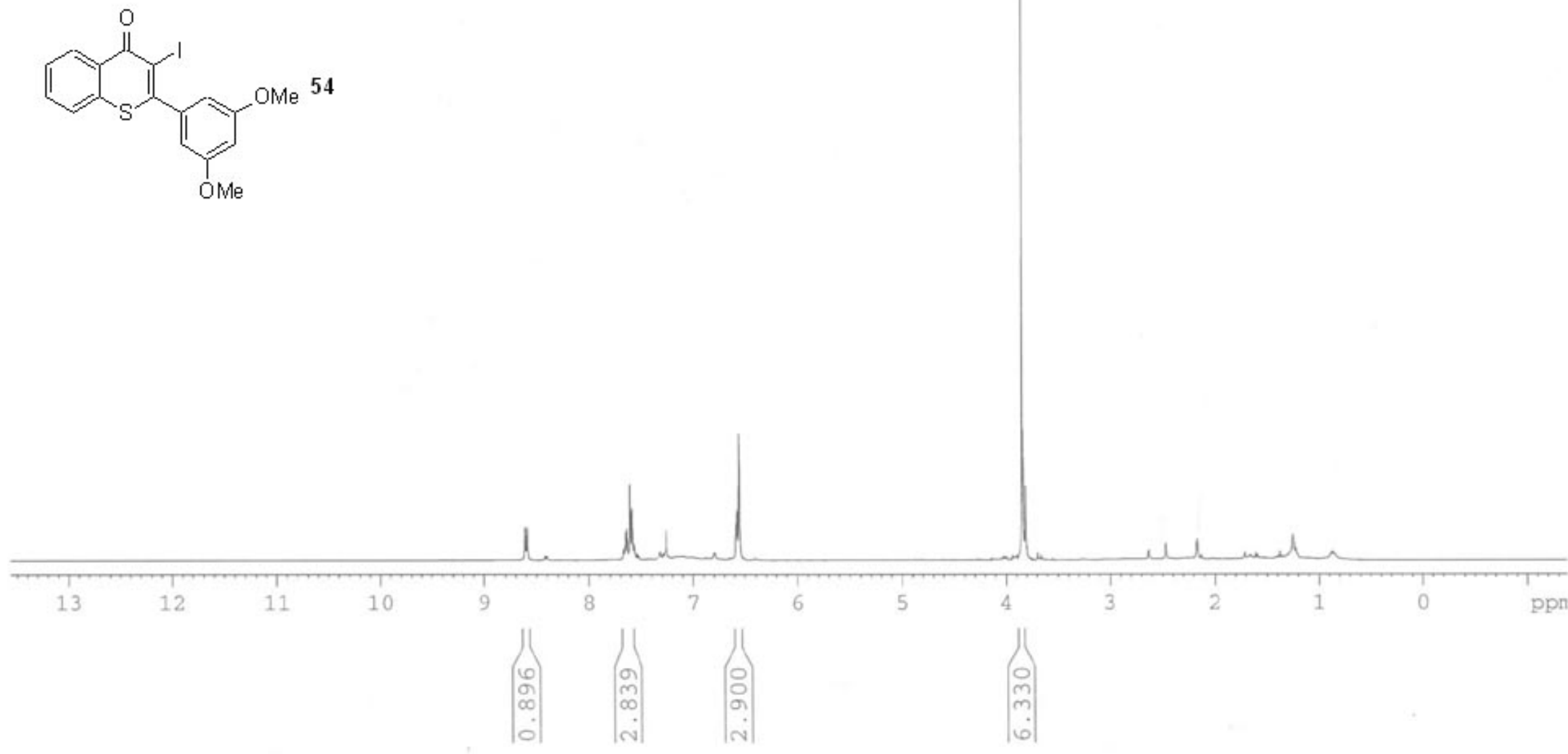

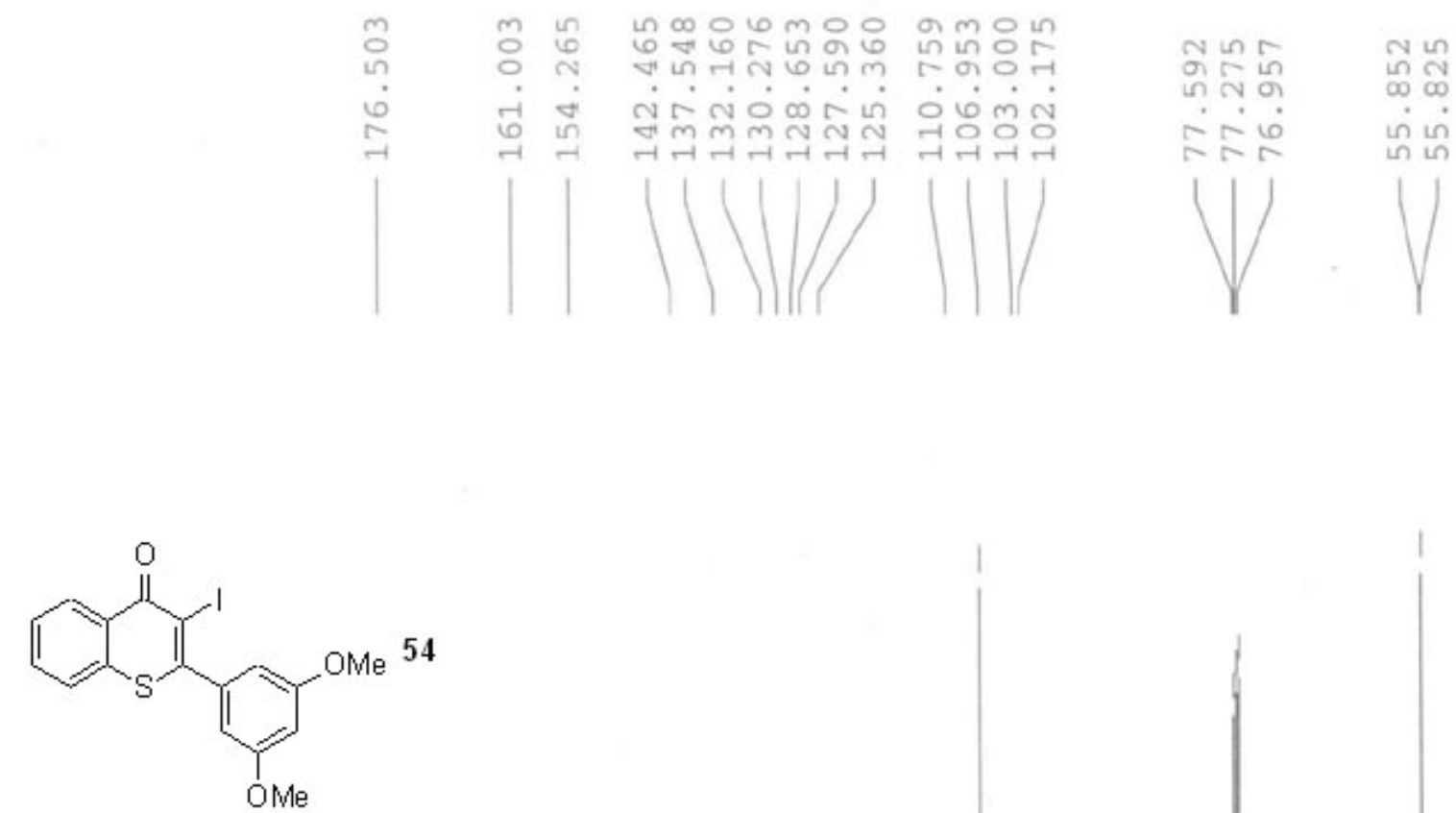
क

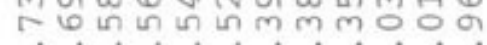

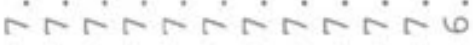

unille

$\stackrel{5}{\infty}$

응

N

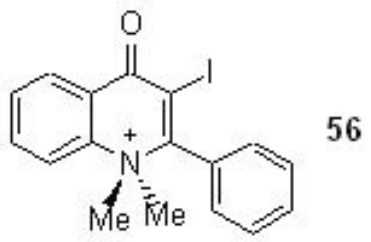

$\mathrm{Cl}$

56

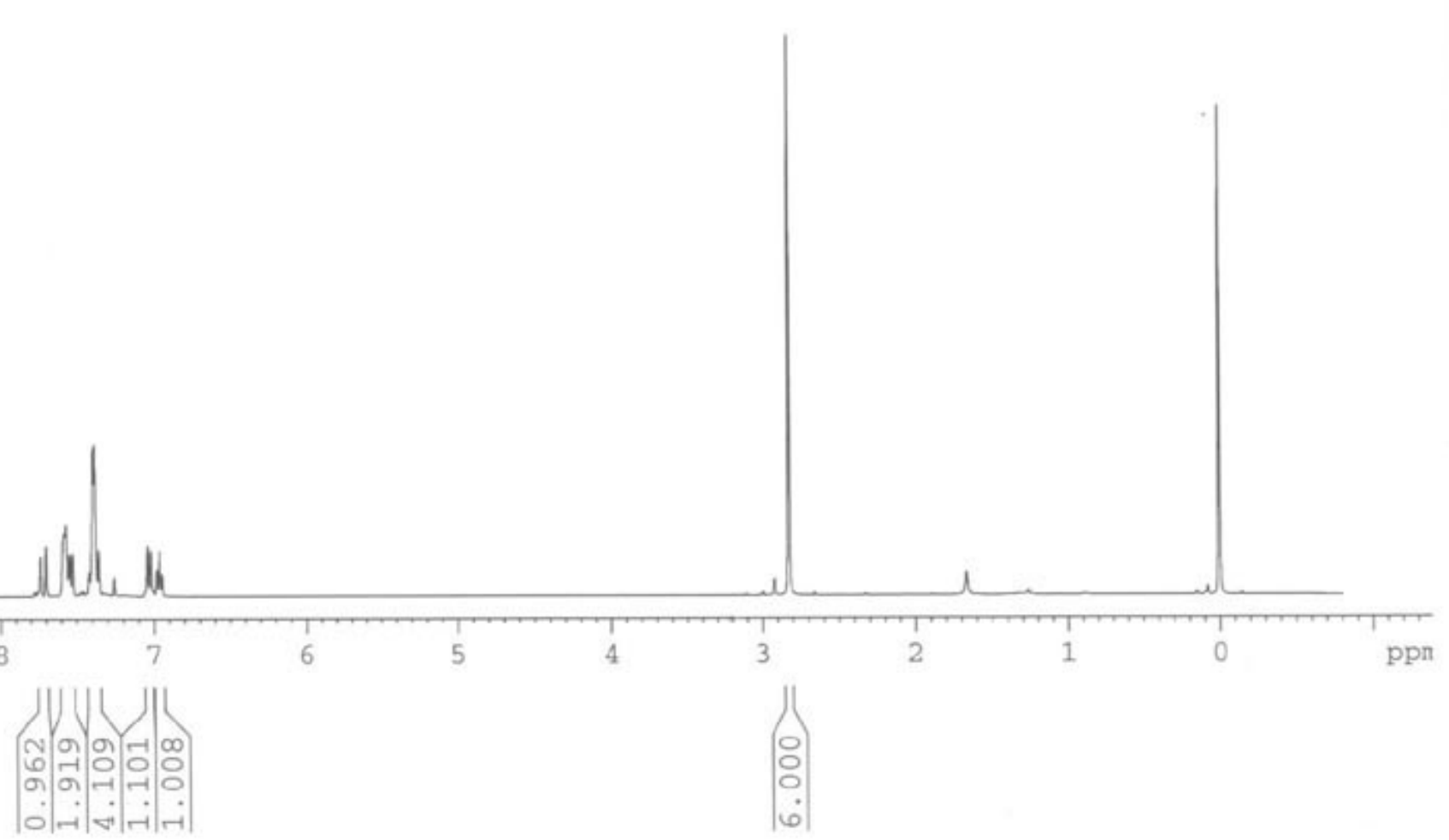



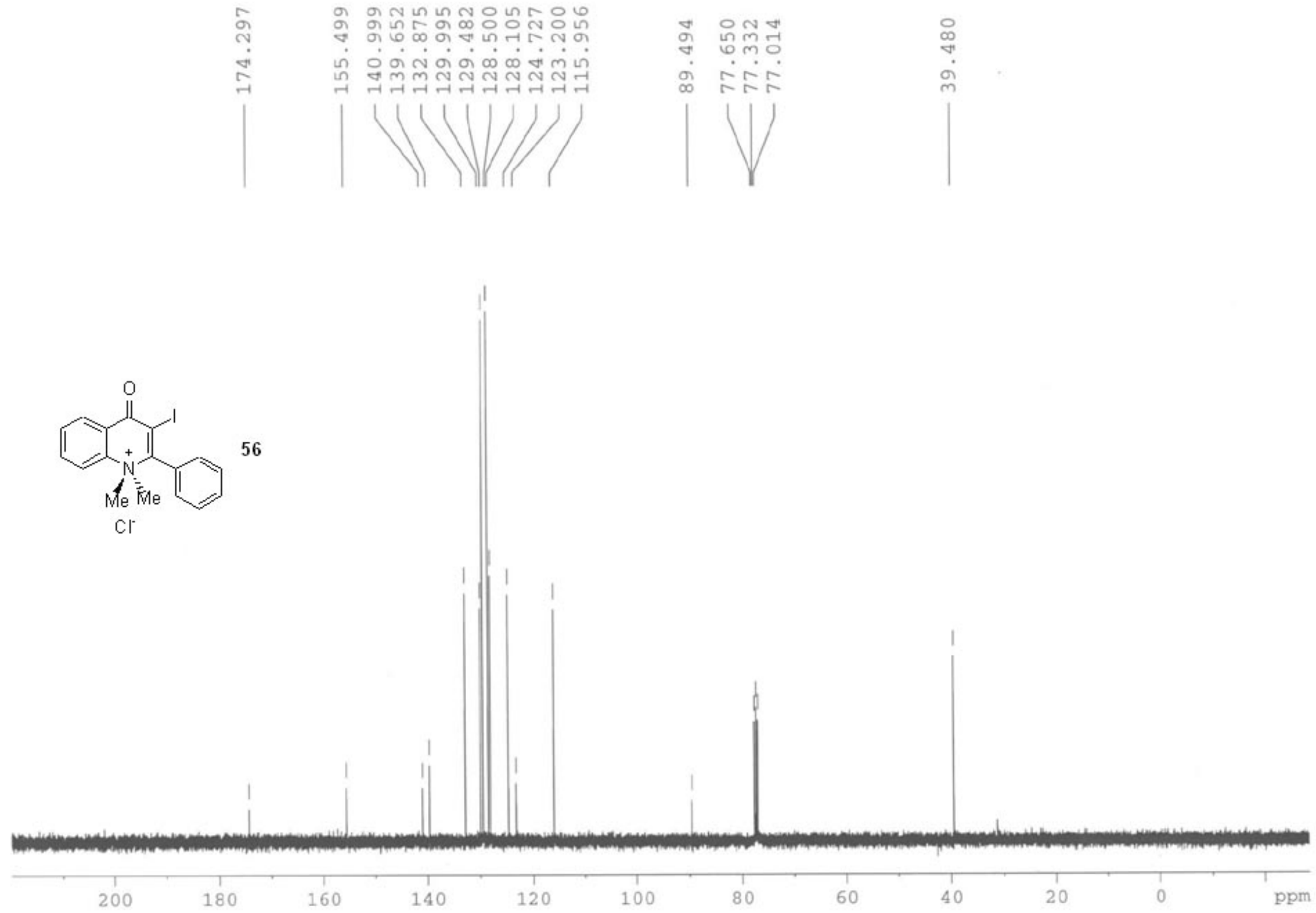


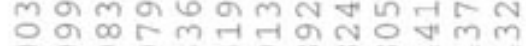
(1) aririnivivivi
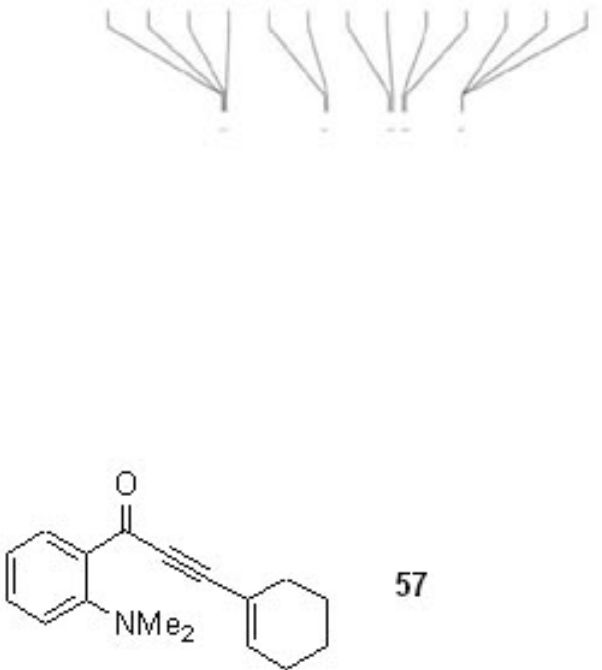

57
궁 以

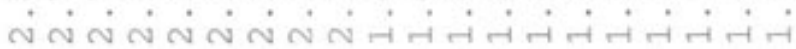

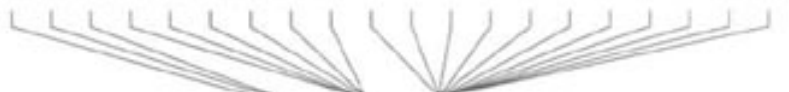

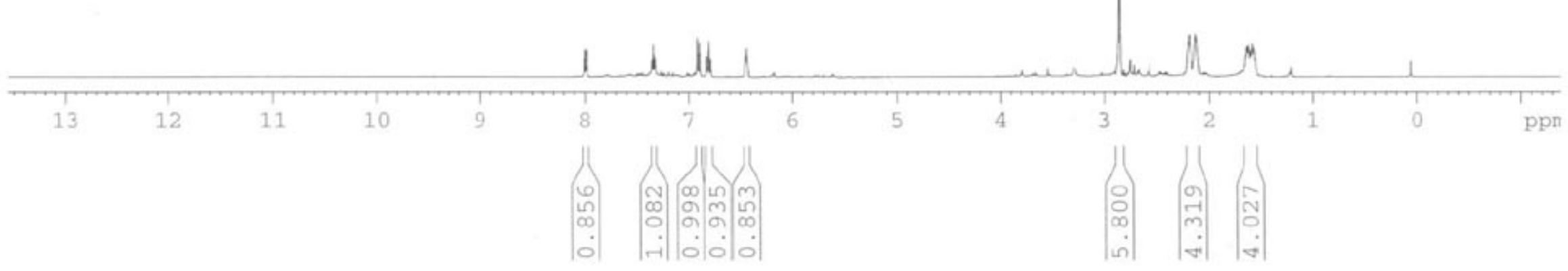



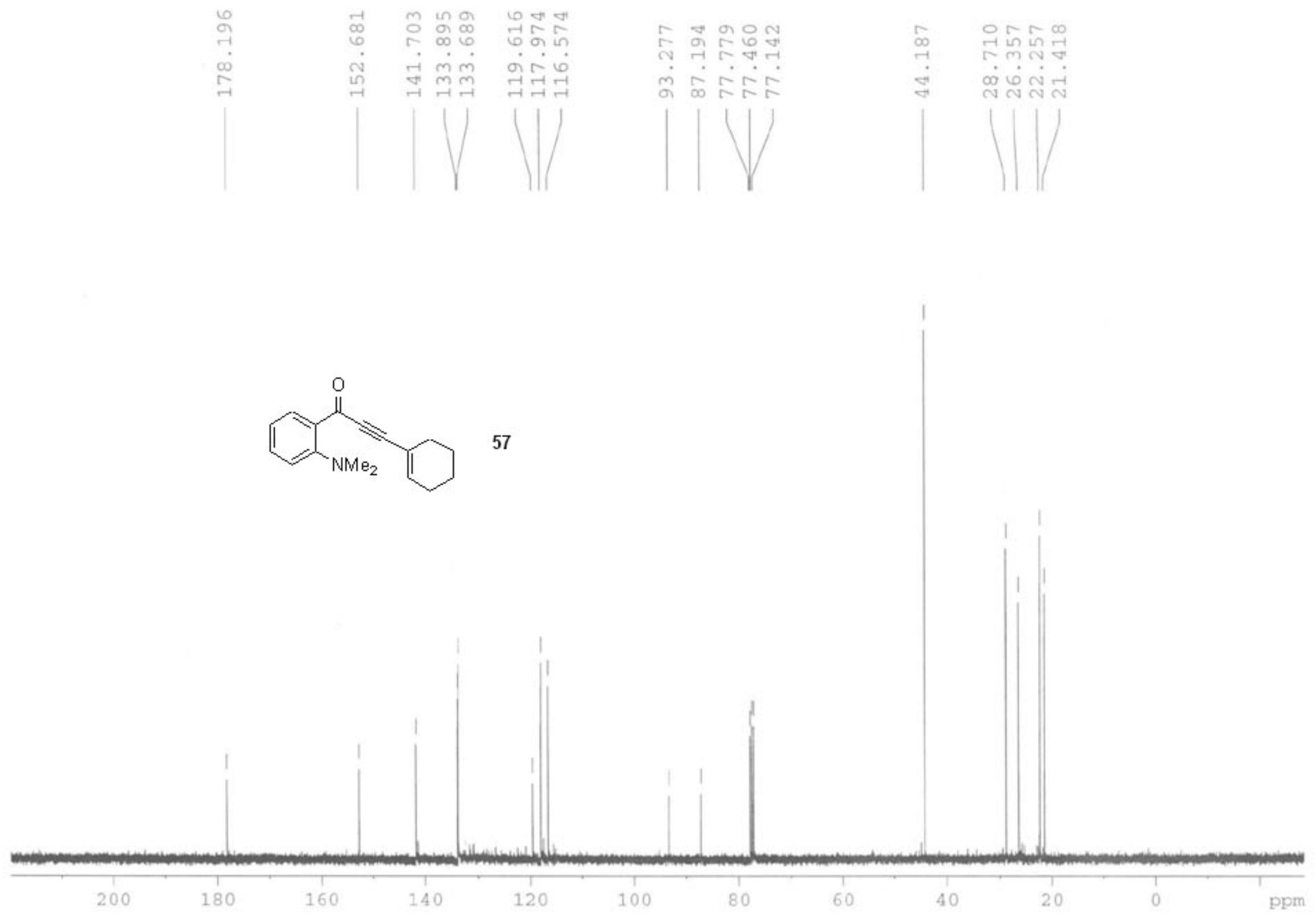
$\infty$ 은ำ แู่

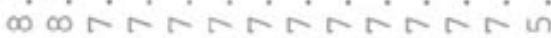
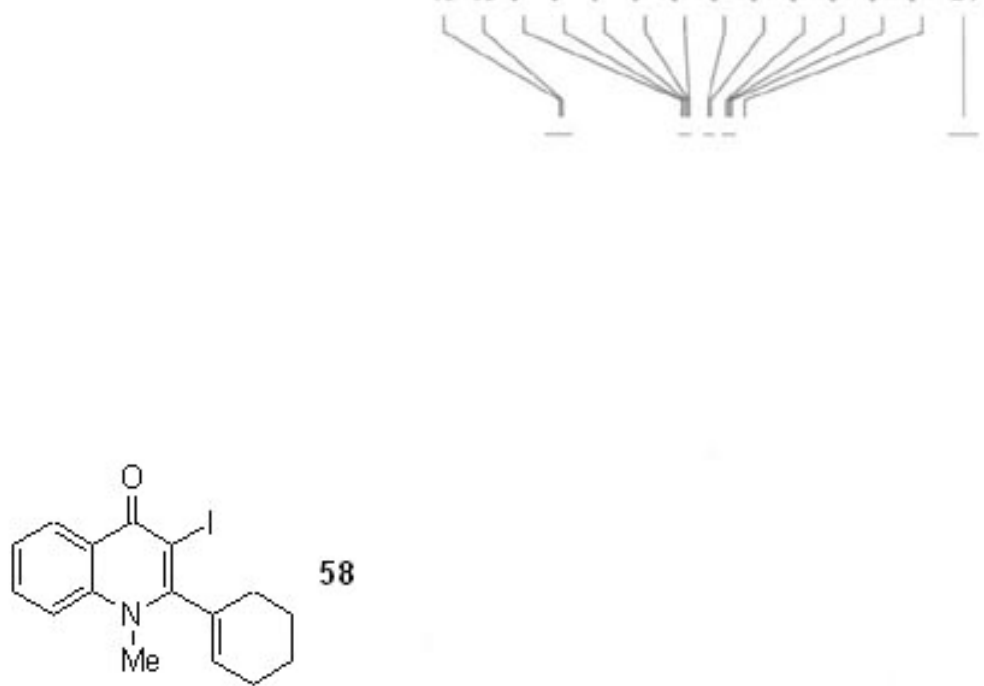

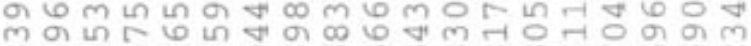
ल

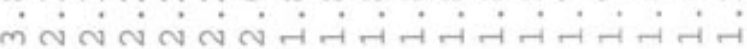

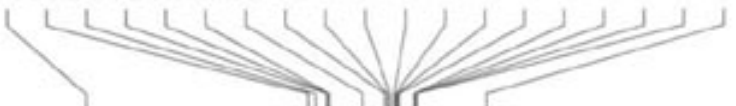

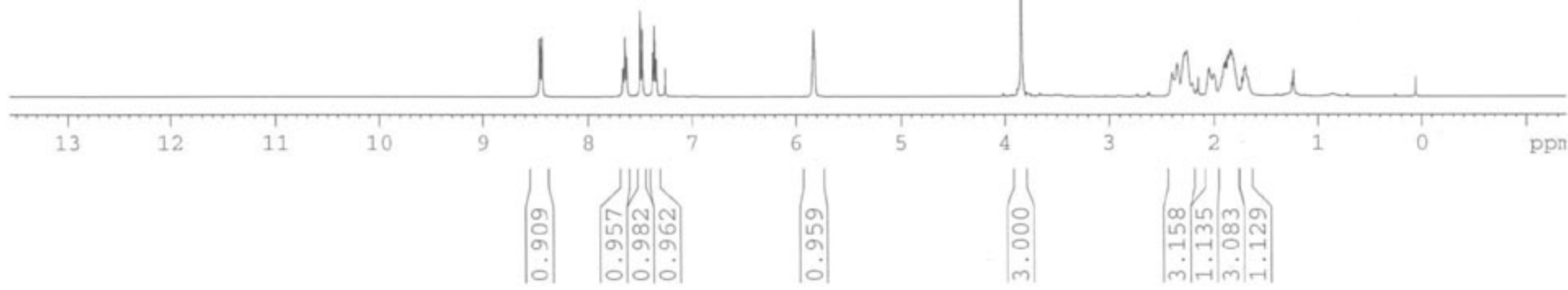



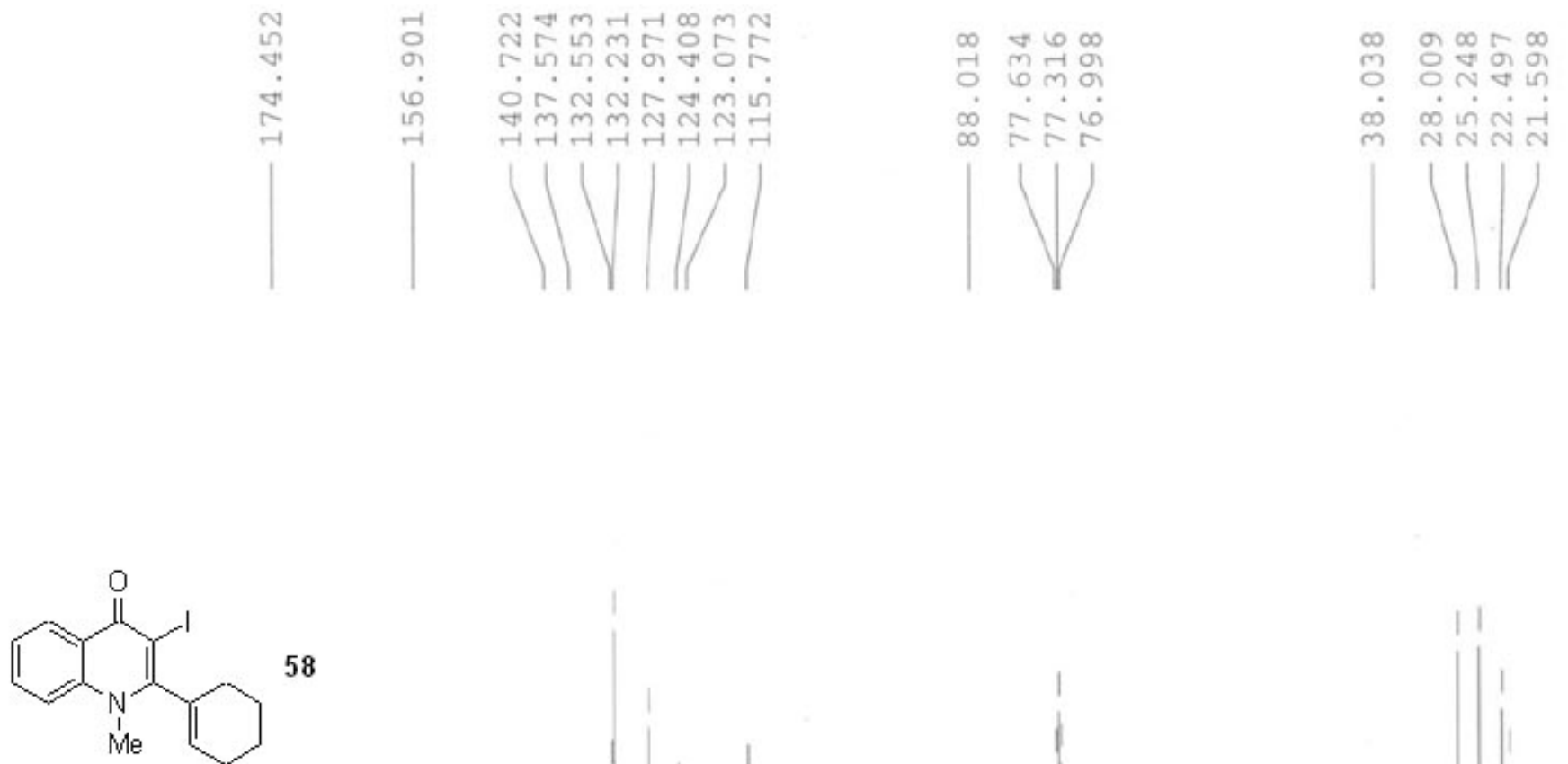

58

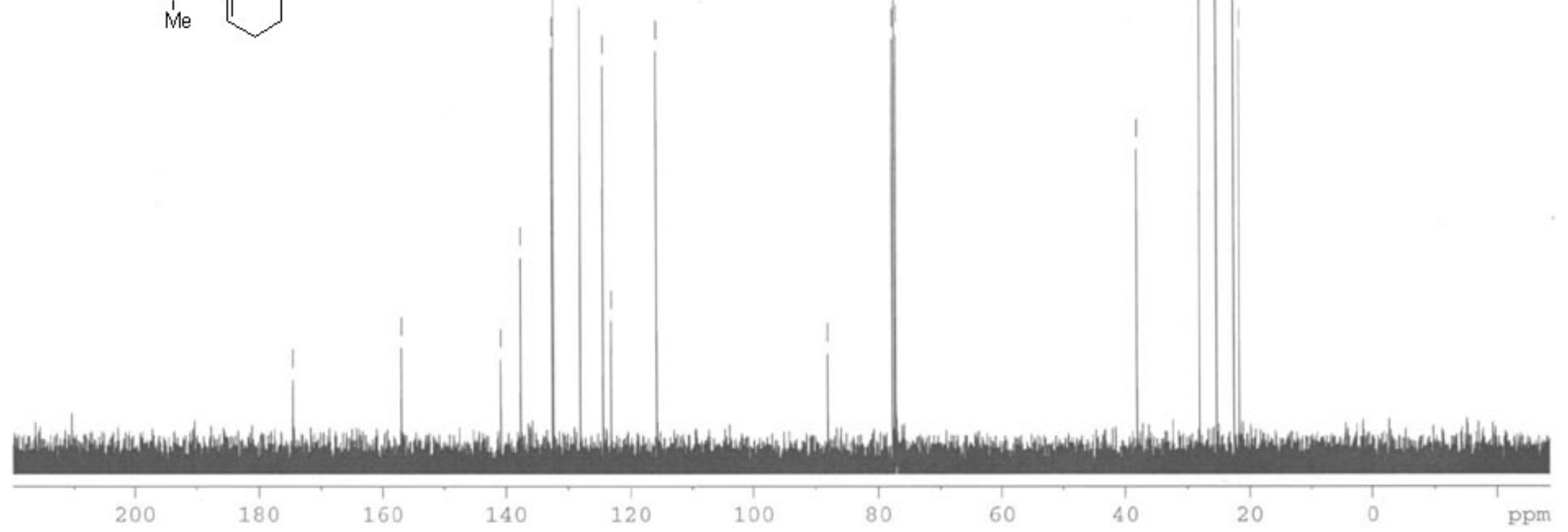


옹ำ

क⿻rín
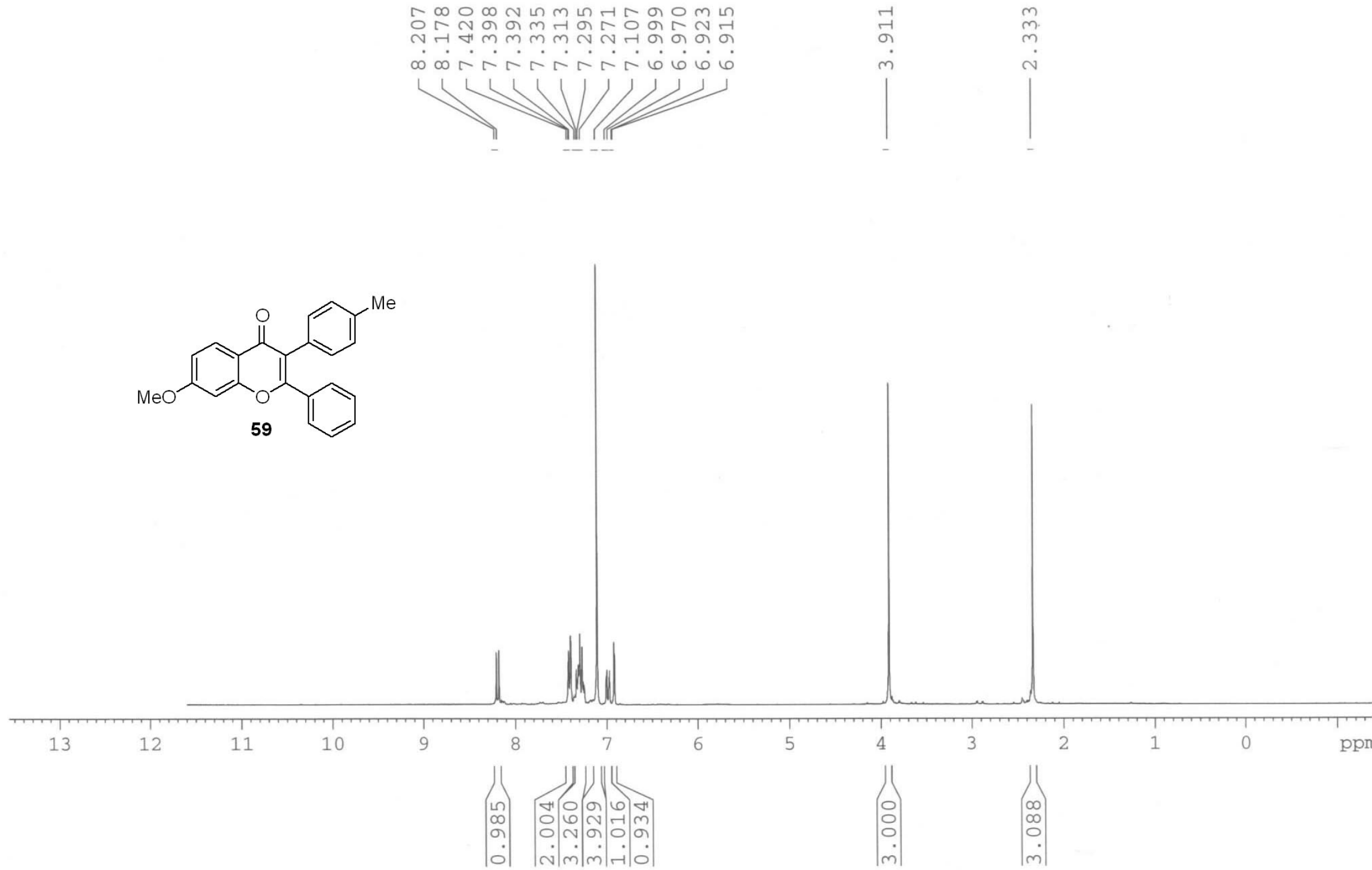


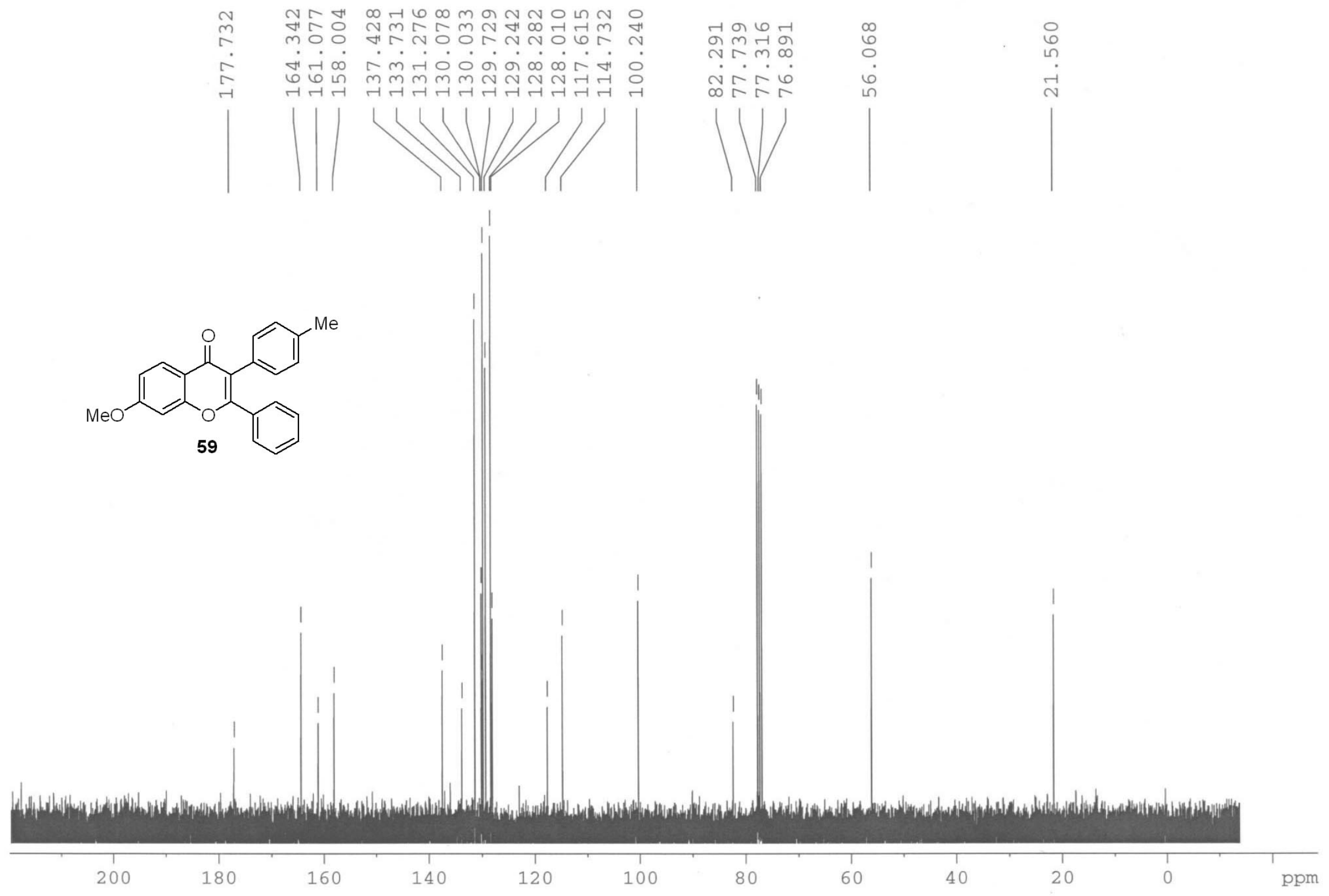


6m $m$ m

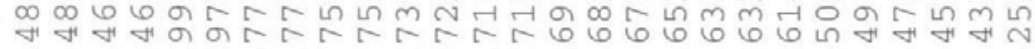

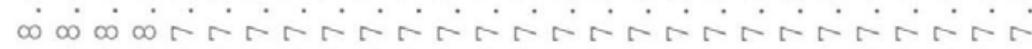

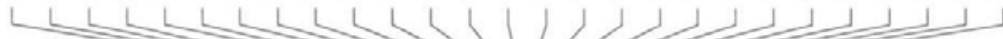
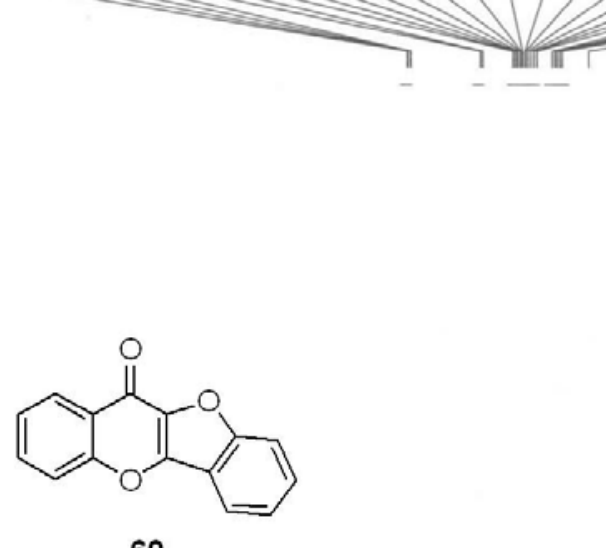

60

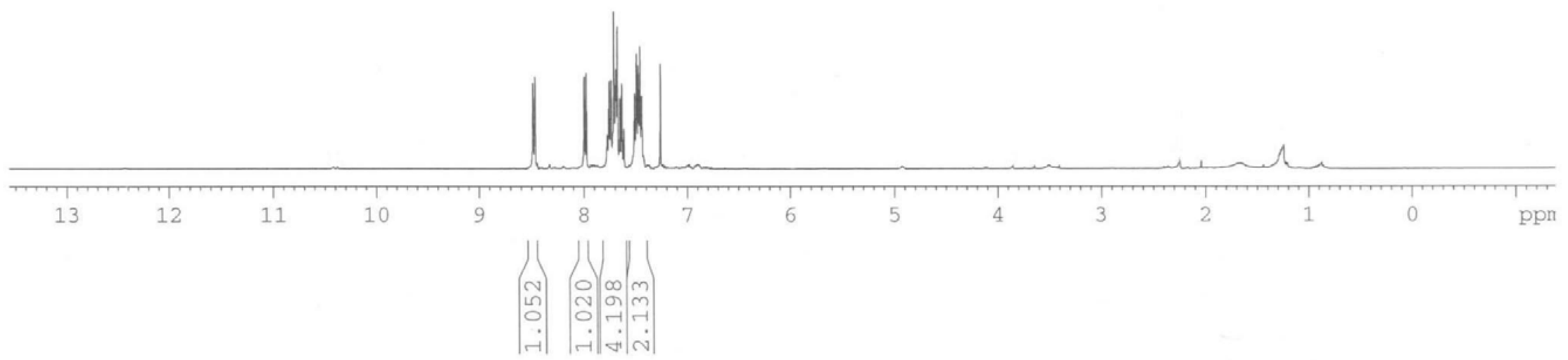



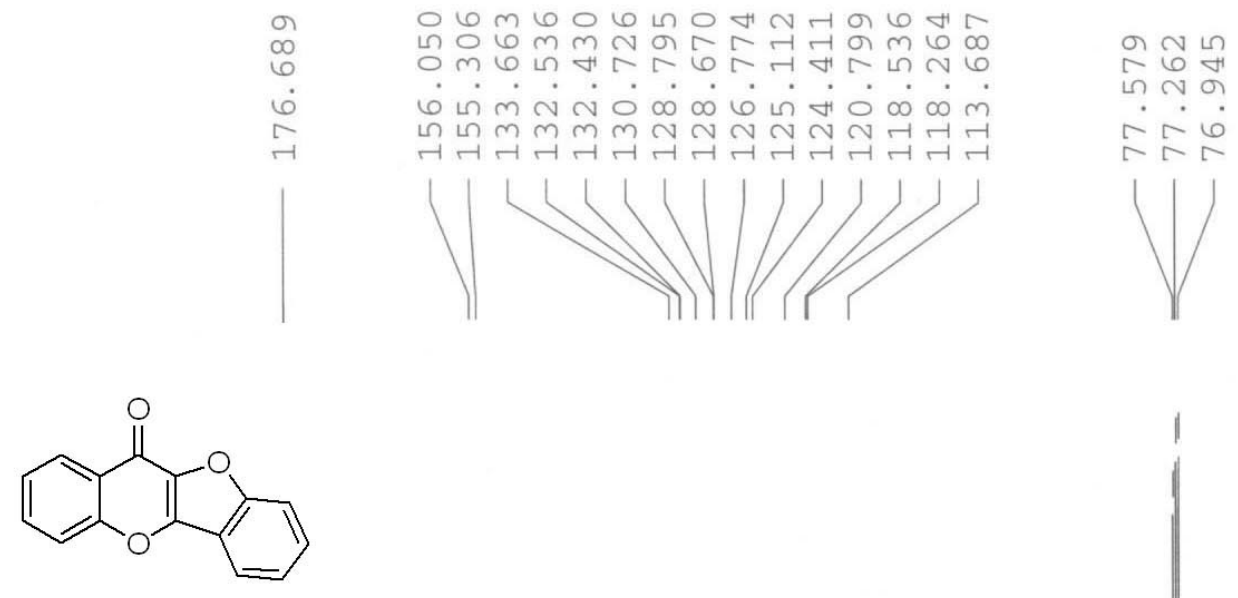

60

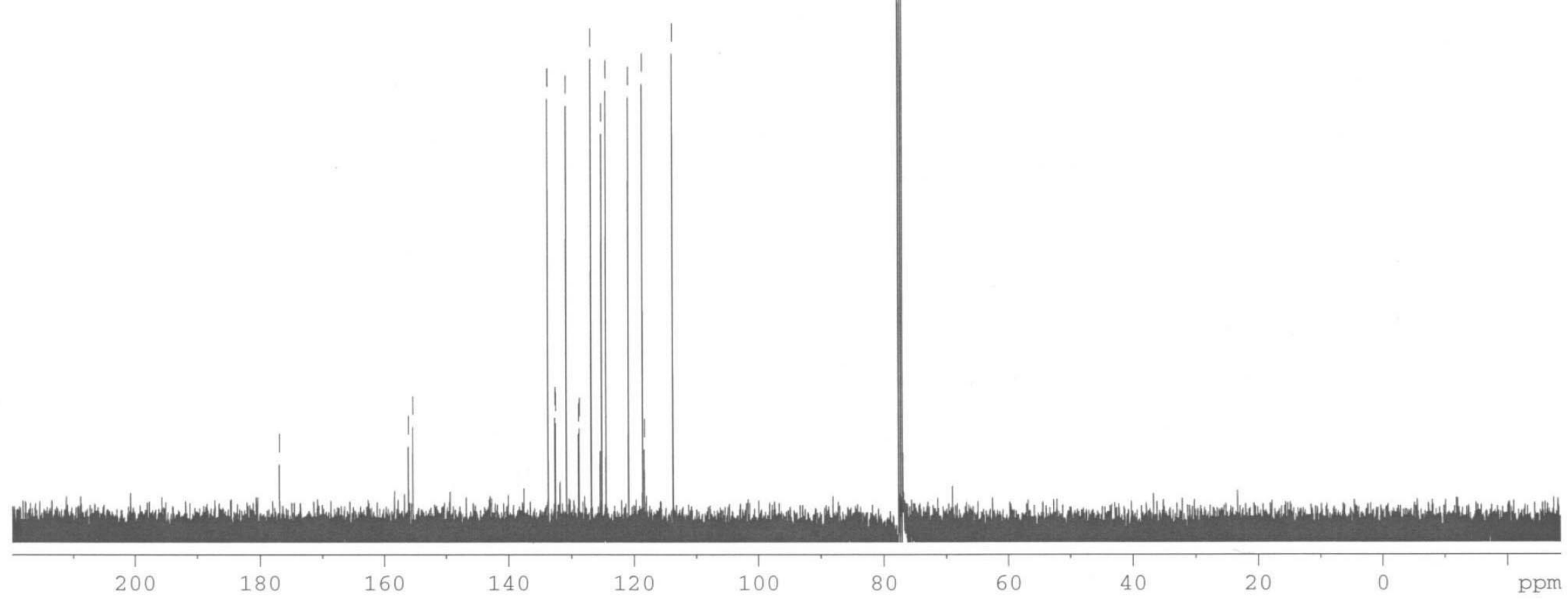

